\title{
Advances and challenges in single-molecule electron transport
}

\author{
Ferdinand Evers \\ Institut für Theoretische Physik, Universität Regensburg, D-93053 Regensburg, Germany \\ Richard Korytár $\odot$ \\ Department of Condensed Matter Physics, Faculty of Mathematics and Physics, \\ Charles University, Ke Karlovu 5, 12116 Praha 2, Czech Republic \\ Sumit Tewariø \\ Huygens-Kamerlingh Onnes Laboratory, Leiden University, \\ Niels Bohrweg 2, 2333 CA Leiden, Netherlands \\ and Department of Materials, University of Oxford, OX1 3PH Oxford, United Kingdom
}

Jan M. van Ruitenbeek $\odot^{\star}$

Huygens-Kamerlingh Onnes Laboratory, Leiden University, Niels Bohrweg 2, 2333 CA Leiden, Netherlands

(published 17 July 2020)

\begin{abstract}
Electronic transport properties of single-molecule junctions have been widely measured by several techniques, including mechanically controllable break junctions, electromigration break junctions, and by means of scanning tunneling microscopes. In parallel, many theoretical tools have been developed and refined for describing such transport properties and for obtaining numerical predictions. Most prominent among these theoretical tools are those based upon density functional theory. In this review, theory and experiment are critically compared, and this confrontation leads to several important conclusions. The theoretically predicted trends nowadays reproduce the experimental findings well for series of molecules with a single well-defined control parameter, such as the length of the molecules. The quantitative agreement between theory and experiment usually is less convincing, however. Two main sources for the quantitative discrepancies can be identified. Experimentally, the atomic structure of the junction typically realized in the measurement is not well known, so simulations rely on plausible scenarios. In theory, correlation effects can be included only in approximations that are difficult to control for experimentally relevant situations. Therefore, one typically expects qualitative agreement with present modeling tools; in exceptional cases a quantitative agreement has already been achieved. For further progress, benchmark systems are required that are sufficiently well defined by experiment to allow quantitative testing of the approximation schemes underlying the theoretical modeling. Several key experiments can be identified suggesting that the present description may even be qualitatively incomplete in some cases. Such key experimental observations and their current models are also discussed here, leading to several suggestions for extensions of the models toward including dynamic image charges, electron correlations, and polaron formation.
\end{abstract}

DOI: 10.1103/RevModPhys.92.035001

\section{CONTENTS}

I. Introduction

II. Experimental Techniques

A. Mechanically controllable break junctions

B. Electromigration break junctions

C. Methods based on scanning probe microscopy

D. Data analysis and conductance histograms

III. Computational Techniques

A. A guided tour through quantum-transport theories

B. Brief overview of electronic-structure calculations for molecular junctions

*ruitenbeek@physics.leidenuniv.nl
C. Verification and validation of transport computations

D. The standard theory of $a b$ initio transport

1. Single-particle aspect, scattering theory, and partioning

2. Discussion of Kohn-Sham transport calculations

3. Proposed improvements over GGA-based Kohn-Sham calculations

4. Discussion of nonlinearities in the $I-V$ characteristics

E. Transport viewed as relaxation and incoherent processes

1. Alternative derivation of the trace formula

2. Eigenchannel decomposition

3. Limit of sequential transport and relation to the Marcus theory of charge transfer 
Evers et al.: Advances and challenges in single-molecule ...

IV. Model-Based Analytical Results

A. Qualitative discussion of few-level models

1. Two-level model without interactions

2. Basics of SIAM, Coulomb blockade, and Kondo effect

3. Two-impurity Anderson model

B. Quantum interference effects

1. Symmetry considerations in orbital representation

2. Sum-over-paths approach

3. Selection rules for destructive QI

4. Applications

5. QI and ring currents

6. Temperature and interaction effects

V. Key Experimental Results and Their Semiquantitative Understanding

A. Conductance as a function of length

1. Basic concepts

2. Conjugation and metallicity

3. Length dependence for conjugated wires

4. Incoherent transport limit

B. Conductance as a function of molecular conformation

C. Anchor groups

1. Thiol-based anchoring groups

2. The role of mechanical coupling

3. Anchor transparency and gateway states

4. Direct metal-molecule coupling

5. Level alignment

D. Quantum interference

E. Electrostatic effects and image charges

F. Current-voltage characteristics

G. Thermal and thermoelectrical properties

H. IETS and sign inversion

I. Coulomb blockade and the Kondo effect

1. The single-impurity Anderson model in single-molecule junctions

2. Two-impurity Anderson model

3. The Kondo effect as evidence for an open-shell structure

J. Franck-Condon blockade

VI. Case Studies of Quantitative Comparison

A. High zero-bias conductance

B. Low zero-bias conductance

C. Intermediate zero-bias conductance

1. Benzenedithiol

2. Alkanedithiols

3. Alternative benchmark systems

4. Concluding remarks

VII. Selected Open Problems

A. Experimental phenomena awaiting basic qualitative understanding

B. Chirality-induced spin selectivity

C. Challenges to theory and modeling

VIII. Conclusions

A. Benchmark systems

B. Uncovering physical phenomena with robustness

C. The important role of DFT-based computations

D. Outlook

1. Precision, reproducibility, and control

2. Toward novel phenomena:

Challenges for experiments

3. Toward time-dependent studies:

Molecular plasmonics
4. Toward devices: CISS and molecular-nuclear spintronics

Acknowledgments

56

References

\section{INTRODUCTION}

Despite many experimental hurdles the understanding of electron transport of single-molecule junctions has seen impressive progress in recent years (Scheer and Cuevas, 2017). It is interesting to observe that it is now routinely possible to wire an organic molecule, an object as small as $1 \mathrm{~nm}$, between two metallic leads and measure its electronic transport characteristics. Several approaches even allow bringing a third metal lead close enough to serve as a gate electrode, through which the conductance of the molecule can be adjusted electrostatically.

Now that we control to some extent the basic properties of molecular junctions, the time is ripe to critically evaluate the question as to how well we understand electron transport in molecular junctions. Faithful modeling inevitably needs to take into account many details of the arrangements of the atoms and the molecule that make up the junction. Since molecular junctions are formed spontaneously under the influence of atomic and molecular interactions, which can be regarded as a form of self-assembly, and since imaging of the resulting structures has not been possible, experiment usually does not provide all of the atomistic information needed for comparison with theory.

Theoretical approaches often employed for describing nearequilibrium electron transport are based on tight-binding methods and density functional theory (DFT) and sometimes also rely on more advanced many-body techniques, such as the $G W$ approximation. Far from equilibrium, i.e., at high voltage bias, the nonequilibrium Green's function (NEGF) method has been widely used. DFT and $G W$ have been amply tested for bulk systems and gas-phase molecules, but molecular junctions pose new challenges. Moreover, suitable variants of the NEGF formalism have been specially developed for these types of problems, which, regretfully, are difficult to benchmark for lack of reliably reference data.

The following question then arises: what is the predictive power of the theories? What are the critical experimental tests? DFT is used widely as a guide for interpreting experiments, but do we know how reliable it is, and how do we know this? How sensitive are the results to the choice of methods and to the assumptions? The problem lies partly in the computational methods themselves, where the level of approximation may be critical, the convergence needs to be controlled, and it needs to be assessed whether the relevant physical mechanisms have been included in the description. On the other hand, when setting up a calculation many assumptions are made about the conditions of the experiments, while the validity of these assumptions in most cases cannot be directly verified from information obtainable from the experiments. Without attempting to be exhaustive in the following, we list a number of items that need to be considered in evaluating a specific molecular junction. 
Molecule-metal binding motifs. - The binding sites of a molecule anchoring on a metal surface and its binding motifs may show high variability. Indeed, the electron transport is sensitive to the atomic structure of the metal at the interface to the molecule (Schull, Frederiksen et al., 2011), to the choice of binding site (e.g., top, hollow, or bridge site), and also to the orientation of the bond with respect to the surface, see the review by Häkkinen (2012). However, in considering the various possible binding configurations it is important to be aware that the experimental conditions are often such that more than just a single molecule is present at or near the specific junction site. Moreover, repeated contact making and breaking, which is widely employed in experiments, may lead to the formation of metal-molecule complexes and produce molecule fragments. Strange, Lopez-Acevedo, and Häkkinen (2010) considered this much wider variability in binding motifs for benzenedithiol ( $\left.\mathrm{HS}-\mathrm{C}_{6} \mathrm{H}_{4}-\mathrm{SH}\right)$ and Au electrodes, leading to a much larger range of computed conductance values than normally considered.

Fluctuating geometries.-Longer molecules, such as the widely studied alkanedithiols [chemical formula $\mathrm{HS}-\left(\mathrm{CH}_{2}\right)_{n}-$ $\mathrm{SH}$, permit even wider variability; see Fig. 1. During the breaking of a junction, the anchoring of the molecule may slide along the surfaces of the two electrodes, and the resulting conductance may vary during this process by more than an order of magnitude (Paulsson et al., 2009). Moreover, the

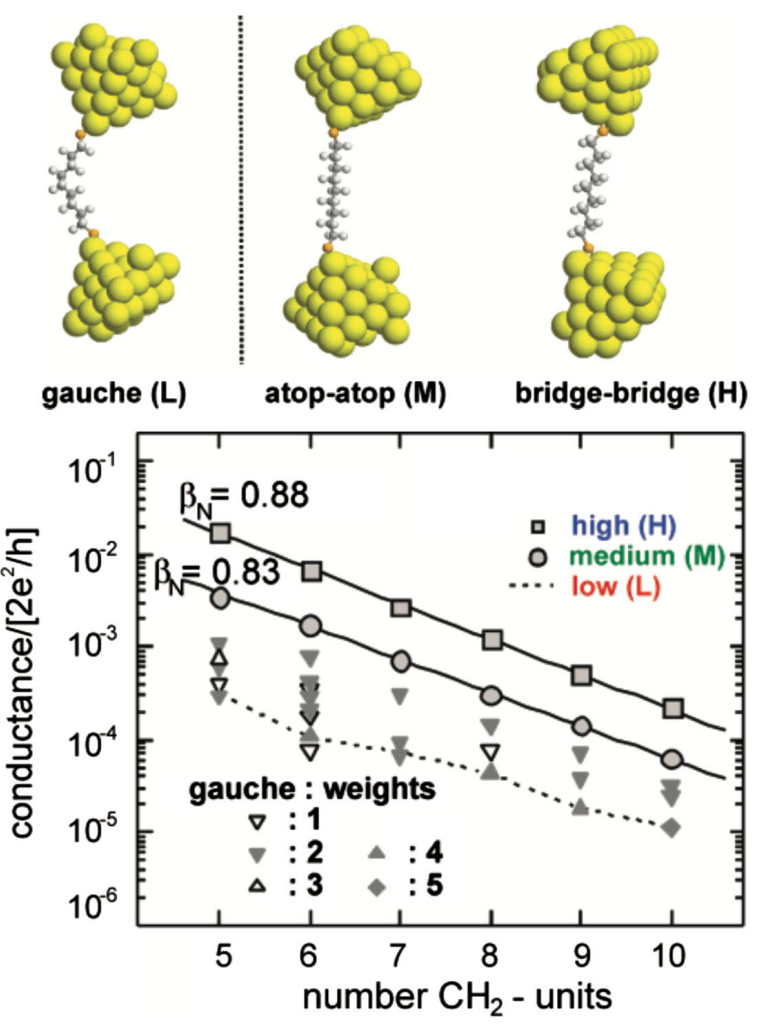

FIG. 1. Relaxed geometries representing three typical arrangements of an alkanedithiol molecule bridged between Au electrodes and calculated length dependence of the conductance for these arrangements. The results illustrate the spreading of conductances that can occur due to structural modifications. From Li et al., 2008. configuration of the molecule has a significant influence on the conductance, depending on the number of gauche defects in the molecular chain (Jones and Troisi, 2007; Li et al., 2008). At room temperature, such defects may form spontaneously and the conductance as measured will be an incoherent time average over the accessible configurations. Dramatic effects of such thermal averaging were shown in calculations (Maul and Wenzel, 2009) for molecular wires containing up to four benzene rings coupled together (oligophenylenedithiol).

Uncertainties of surface chemistry and level alignments.The nature of the chemical bond between the molecule and the metal electrodes is another source of ambiguity. The widely exploited Au-S-R anchoring, where $R$ is the molecular group under study, is often obtained by adding thiol $(\mathrm{SH})$ end groups to the molecule. In the process of binding to $\mathrm{Au}$ one usually assumes that the hydrogen atom is split off and removed, but evidence suggests otherwise (Stokbro et al., 2003; Inkpen et al., 2018). Just as hydrogen remaining at or near the anchoring group, the presence of other residuals or entire molecules on the surface also has further consequences. Such surface coverage modifies the metal work function and thus modifies the profile of the electrical potential drop along the junction axis. A dramatic demonstration of this effect was given in the experiments by Capozzi et al. (2015). When working in solution the ions in the electrolyte dynamically adjust to the applied bias voltage, producing an asymmetric diodelike current-voltage $(I-V)$ characteristic. Size and shape of the electrodes on the nanometer scale also affect the details of the electron transport (Häkkinen, 2012) and the profile of the electrical potential drop (Brandbyge, Kobayashi, and Tsukada, 1999). Information on such nanoscale details is not readily obtained from the experiment. One reason for the sensitivity of electron transport to the nanoscale shape of the electrodes is the effect of image charges (Perrin et al., 2013).

Electron transport for metal-molecule-metal junctions is typically off resonant, which makes the conductance highly sensitive to the energy of the delocalized molecular orbital nearest to the Fermi level of the electrodes. This position is influenced by many of the factors listed previously, and in addition this position self-adjusts by partial charge transfer between the metal and the molecule.

Is our description complete?-Given these many poorly known factors one should conclude, as we see later, that the agreement between experiment and computations is surprisingly good. To be more precise, conductance values for the same metal-molecule combinations and most calculations find an agreement within an order of magnitude from the experiment (although there are important exceptions, as we see later). This raises three interesting questions: (i) Given the many unknowns, why is the agreement so close? (ii) If we could improve our knowledge of the experimental system to be described, how strong would the predictive power of theory be? (iii) Are we possibly missing some interesting physics in the description?

The last question is the most important, in our view. For example, the interplay between the bias voltage, electrode screening, and Coulomb blockade can introduce nontrivial correlation effects, such as a negative-differential conductance (Kaasbjerg and Flensberg, 2011). This regime escapes the single-particle doctrines and has hardly been explored. 
Electrons also interact with the ion cores by means of vibrations, leading to inelastic scattering signals that can be exploited for characterizing the molecular junction (Smit et al., 2002). The associated limit of strong electron-lattice interactions was reviewed by Thoss and Evers (2018). It is expected to lead to polaron formation ( $\mathrm{Su}$, Schrieffer, and Heeger, 1980), which should have a strong impact on the current-voltage characteristics (Galperin, Ratner, and Nitzan, 2005; Thoss and Evers, 2018). Recently this mechanism has been shown explicitly in experiments, although the result was obtained not for a typical molecular junction but rather for a molecule in a scanning tunneling microscope (STM) tunneling configuration (Fatayer et al., 2018).

Structure of this review.-Single-molecule transport is an extremely active and broad research field with a corresponding body of literature. A single review cannot hope to do full justice to all developments, even when focusing on a few relevant aspects. It is our aim in this review to summarize and discuss the most significant experimental and theoretical results in the light of the set of specific questions raised earlier. In particular, we critically evaluate the level of agreement between theory and experiment. We further elaborate on selected experiments and calculations in the review that indicate that the description of the systems may not be complete, and that suggest interesting physics beyond the standard approaches. For comprehensive reviews focusing on complementary aspects of molecular-scale transport, we refer the interested reader to $\mathrm{Su}$ et al. (2016), Jeong et al. (2017), Scheer and Cuevas (2017), and Thoss and Evers (2018). While our focus is on single-molecule junctions, we occasionally also quote results obtained for self-assembled monolayers.

\section{EXPERIMENTAL TECHNIQUES}

In this section, we present various techniques used for studying electronic transport through single molecules to acquaint the reader with the methods that we encounter while discussing the results. For a more detailed presentation of single-molecule techniques and their integration into various advanced measurement schemes, we refer to previous reviews (Agraï, Levy Yeyati, and van Ruitenbeek, 2003; Aradhya and Venkataraman, 2013; Xiang et al., 2013, 2016).

Since molecules have a typical size of $1 \mathrm{~nm}$, all existing top-down microfabrication techniques lack the required resolution for controlled wiring of molecules. Therefore, the methods employed rely on a combination of electromechanical fine-tuning of the nanometer-size gap between the contact electrodes and self-assembly of the molecules inside this gap. The three most frequently employed techniques are the mechanically controlled break-junction (MCBJ) technique, the electromigration break junction technique and methods using STMs.

\section{A. Mechanically controllable break junctions}

The MCBJ technique was developed for the study of atomic and molecular junctions (Muller, van Ruitenbeek, and de Jongh, 1992) based on an earlier method aimed at studying vacuum tunneling between superconductors (Moreland et al.,
1983). We distinguish between two fabrication methods: the notched-wire MCBJ and the lithographically fabricated MCBJ. The first is simpler and has the advantage that it can be easily adapted to nearly all metal electrodes. It is made starting from a macroscopic metal wire into which a weak spot is created by cutting a notch. The notched metal wire is placed on top of a flexible substrate (which is commonly stainless steel or phosphorous bronze) covered by an insulating sheet, usually Kapton. The wire is fixed by epoxy onto the substrate at either side and close to the notch. This is then mounted in a three-point bending mechanism as shown in Fig. 2(a). Bending the substrate increases strain on the wire, which is concentrated at the weak spot created by the notch, until the wire breaks. The junction is first broken with a coarse mechanical drive, thereby exposing two fresh electrode surfaces. By relaxing the bending and using fine control of the gap by means of a piezoelectric actuator, atomic-size contacts can be reformed and broken many times.

The lithographically fabricated MCBJ (van Ruitenbeek et al., 1996) shares the same principle as the notched-wire MCBJ except that the prenotched metal wire is replaced by a freely suspended bridge in a thin metal film produced by electron-beam lithography. This metal film is electrically isolated from the flexible substrate using a $3-5 \mu \mathrm{m}$ polyimide layer. The unsupported section of the bridge is reduced by about 2 orders of magnitude compared to the notched-wire MCBJ, to about $2 \mu \mathrm{m}$, or less. This has the effect that the mechanical displacement ratio, i.e., the ratio between the change of the gap size and the actuator motion, is reduced to about $10^{-5}$. The gain of using the lithographic technique is that the junctions are insensitive to external mechanical perturbations as a result of the small displacement ratio. The added complications of clean-room preparation are offset by the possibility of producing multiple MCBJ samples on a single wafer (Martin, Ding, van der Zant, and van Ruitenbeek, 2008). A drawback is the fact that by the extremely small
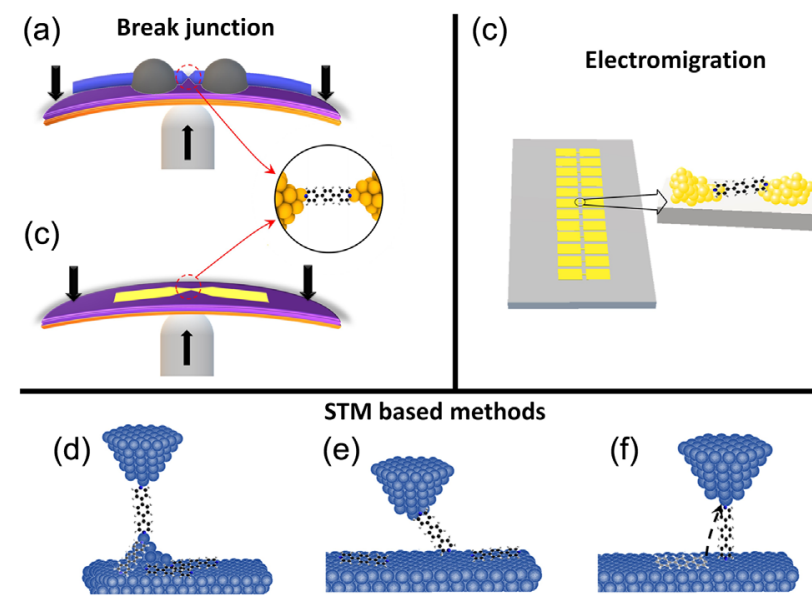

FIG. 2. Overview of experimental techniques aimed at measuring single-molecule transport. (a) Notched-wire mechanically controllable break junction. (b) Lithographically fabricated mechanically controllable break junction. (c) Electromigration break junctions. (d) STM break junction by repeated indentation. (e) $I(t)$ or $I(s)$ operation of STM. (f) Low-temperature UHV STM manipulation of individual molecules. 
displacement ratio the maximum extension of a typical piezoelectric actuator produces a less than $0.01 \mathrm{~nm}$ change in the distance between the electrodes. Therefore, the control of this distance is achieved by an electromotor-driven gear. Since such electromechanical control is much slower than piezoelectrical control, it is much more time consuming to obtain enough statistics for a large number of contact-breaking events (discussed later).

For most types of metal electrodes, one can only take full advantage of the MCBJ method by performing the first breaking at cryogenic temperatures or under ultrahigh vacuum (UHV). Otherwise, the surfaces are contaminated with oxides and adsorbents cover the surface within a fraction of a second, so the atomic-size contact characteristics of the pure metal are lost. The main exception is Au, for which even under ambient conditions most of the intrinsic quantum conductance properties survive as a result of the low reactivity of the Au surface (Pascual et al., 1993).

For the same reason Au stands out as the preferred electrode material for all other single-molecule transport experiments. Specific binding to target molecules can be achieved by selecting suitable anchor groups for the molecules; see also Sec. V.C. Typically, such molecules having suitable anchor groups are deposited onto the bridge of the MCBJ from solution under ambient conditions. This strategy was first explored for lithographic MCBJ systems (Reed et al., 1997; Reichert et al., 2002), and this continues to be the most commonly employed approach, but recently it has also been demonstrated for the notched-wire MCBJ technique (Bopp et al., 2017).

The intrinsic cleanliness of the broken metal surfaces can be more fully exploited by working under UHV and/or under cryogenic conditions. The deposition of molecules in these experiments proceeds by deposition onto the broken junction from the gas phase, either using an external vapor source (Smit et al., 2002; Kiguchi et al., 2008) or employing a local cell for sublimation (Kaneko et al., 2013; Rakhmilevitch et al., 2014). By working under cryogenic or UHV conditions, it is possible to explore other metal electrodes and other forms of metalmolecule bonding. For example, hydrogen $\left(\mathrm{H}_{2}\right)$ binds to clean Pt electrodes without the need for anchoring groups (Smit et al., 2002), and this applies more widely to many organic molecules, such as benzene (Kiguchi et al., 2008), oligoacenes (Yelin et al., 2016), and pyrazine (Kaneko et al., 2013).

\section{B. Electromigration break junctions}

Electromigration in metals (Ho and Kwok, 1989) results from an atom diffusion process driven by the "electron wind" force (Huntington and Grone, 1961) exerted by the conducting electrons on the atoms in the system under large current bias. This effect can be used to create nanogaps in metallic leads (Park et al., 1999; van der Zant et al., 2006) small enough for a single molecule to bridge. Such systems are prepared by first prepatterning a narrow metal wire of about $100 \mathrm{~nm}$ in a thin metallic film on an insulating substrate (usually $\mathrm{SiO}_{2}$ on a $\mathrm{Si}$ wafer) using electron-beam lithography. Passing a large current through such narrow metallic leads gives rise to displacement of atoms, which is observed as increasing resistance due to the gradual thinning of the wire. Initially, the reliability of the method was compromised by the fact that the strong local Joule heating leads to the formation of metallic nanoparticles in almost $30 \%$ of the junctions (Houck et al., 2005; van der Zant et al., 2006), which gives rise to $I-V$ characteristics resembling those of molecules. However, by using a feedback circuit the electromigration process can be more precisely controlled, and further improvements are obtained by relying on self-breaking in the last stages of gap formation (van der Zant et al., 2006).

Molecules are deposited onto the nanowire before electromigration, and one relies on a molecule finding its way into the gap during the electromigration process. Alternatively, molecules can be allowed to self-assemble into the gap from solution after the electromigration process has been completed (Osorio, O'Neill, Wegewijs et al., 2007). In contrast to other break-junction techniques, junctions formed by electromigration can be broken only once and cannot be reformed. The gap distance depends on the details of the feedback-controlled breaking process, but it cannot be targeted precisely. One cannot obtain a precise value for the size of the gap, but a fair estimate can be obtained from fitting the $I-V$ characteristics to the Simmons model (Simmons, 1963; Vilan, 2007).

For imaging techniques, the gap is better accessible than for any of the other techniques discussed here. High-resolution transmission electron microscopy imaging using transparent $\mathrm{SiN}_{x}$ membranes was performed for gold electromigration junctions (Strachan et al., 2008; Gao et al., 2009) to study the breaking process and detect the nanogap size. The imaging resolution of transmission electron microscopy has not yet proven to be sufficient for detecting the position of an organic molecule.

The search for junctions bridged by a molecule is based on producing many (of the order of several hundred) electromigration break junctions on a wafer, breaking each of them separately and probing the resulting junctions for interesting $I-V$ characteristics at room temperature, which may point to the presence of a molecule in the bridge. Such junctions, which are a minority of the order of a few percent, are then further studied, usually by more elaborate techniques. Although the method intrinsically allows one to obtain only limited statistics over molecular junction configurations and every junction formed has its particular characteristics, the more elaborate experiments permit interesting case studies. Moreover, the rigid attachment of the electrodes to the substrate allows temperature and field cycling, it allows the fabrication of a metallic gate at close proximity to the junction (Park et al., 1999; van der Zant et al., 2006), and it permits easy optical access for Raman scattering (Ward et al., 2008).

\section{Methods based on scanning probe microscopy}

The previously described break-junction methods do not permit imaging of the molecule in the junction. In contrast, STM or atomic force microscopy allow imaging molecules on a surface before contacting them. This is possible only for extremely stable systems under UHV (Joachim et al., 1995; Langlais et al., 1999), especially at cryogenic temperatures (Néel et al., 2007; Temirov et al., 2008). By imaging and manipulating single molecules on an atomically flat and clean 

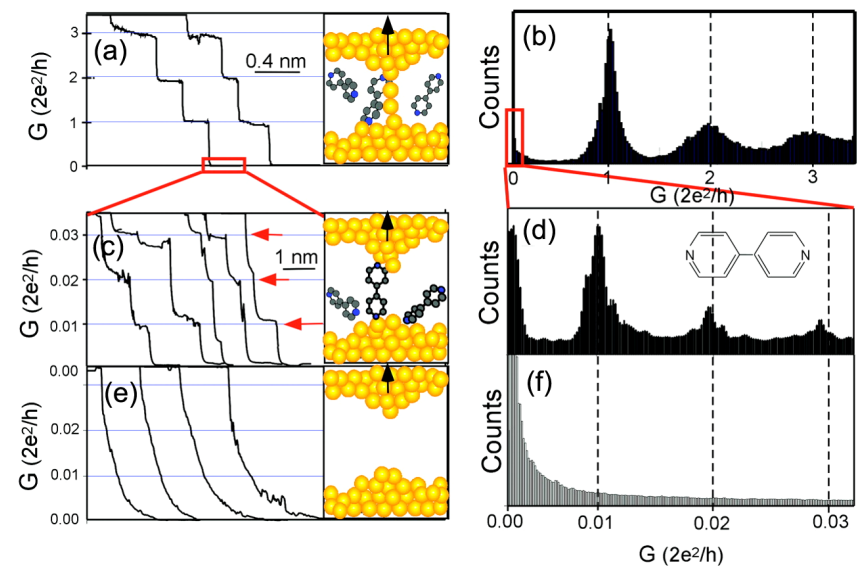

FIG. 3. Experiment probing the conductance of a single molecule by repeated indentation of a Au STM tip into the Au metal surface, in solution of 4,4-bipyridine. The breaking of the metal-metal contact is observed as steps in the conductance (a) near multiples of $G_{0}$, giving rise to (b) peaks in the conductance histogram. (c) Enlarging to lower conductance, additional steps are resolved, and (d) for many repeats of breaking this produces a new series of peaks in the conductance histograms at small fractions of $G_{0}$. (e),(f) Tests with pure solvent show only tunneling characteristics. From Xu and Tao, 2003.

metal surface it is possible to verify that the STM tip interacts with a single target molecule, and the shape of the bottom electrode contacting the molecule (the metal surface) is known. However, information on the shape of the tip cannot be easily obtained from experiments. ${ }^{1}$ Moreover, when approaching the tip for contacting the molecule and lifting it up from the surface, the molecule and the metal atoms contacting it rearrange in ways that cannot be seen by the instrument.

While cryogenic UHV STM holds great promise, it is also a demanding technique. A versatile method for investigating the conductance of single molecules by STM at room temperature and in solution was introduced by $\mathrm{Xu}$ and Tao (2003), and it has inspired many other researchers; see Fig. 3. Ignoring the scanning capability of STM, the instrument is used for approaching the tip to the surface and repeatedly indenting the tip into the surface and retracting. In this mode of operation the atomic structure of the junction is subject to fluctuations, so the information obtained with this technique is statistical in nature, i.e., ensemble based, and thus close in spirit to MCBJ experiments. The indentation of the Au tip into the $\mathrm{Au}$ metal surface to a depth corresponding to a conductance of 10-40 times the conductance quantum $\left(G_{0}=2 e^{2} / h\right)$ restructures the shape of the electrodes with every indentation. Upon retraction a neck is formed that thins until it snaps. The resulting gap is then frequently bridged by a molecule equipped with suitable anchoring groups through a selforganization process, which is observed as a plateau in the

\footnotetext{
${ }^{1}$ Progress in this direction has been made recently in atomic force microscopy (AFM) with CO molecules on Cu tips. The symmetry of the AFM data reveals the structure of the second layer of $\mathrm{Cu}$ atoms that the apex atom couples to (Welker and Giessibl, 2012).
}

conductance during retraction. These plateaus usually have a lot of structure and appear at different levels for each retraction event. Therefore, the indentation and retraction cycles are repeated many times and the resulting conductance traces are combined in the form of conductance histograms, as had been previously introduced for MCBJ experiments (Krans et al., 1993; Smit et al., 2002).

These room-temperature experiments have a significant advantage in that they permit evaluating single-molecule junctions much faster than other available techniques and thereby allow one to explore trends as a function of molecular composition. On the other hand, the information obtained is limited mostly to statistical properties, such as average and typical values of the conductance, the breaking length (Chen et al., 2006), the force holding the junction together (Xu and Tao, 2003; Aradhya and Venkataraman, 2013), and the thermopower (Reddy et al., 2007).

\section{Data analysis and conductance histograms}

Most of the MCBJ and STM experiments have in common that as a result of the self-arranging process involved in the formation of the junction little is known about the atomicscale shape or structure of the electrodes, the configuration of the molecule in the junction, or its bonds to metal surfaces. As a result, the conductance can fluctuate from one contactbreaking trace to the next by an order of magnitude or more. Note that even for a given trace the current at fixed dc voltage is usually not time independent due to thermal or bias-induced fluctuations in the junction geometry. For example, during the process of breaking up a molecular junction in MCBJ or STM-BJ experiments, which can take place on timescales between about $1 \mathrm{~ms}$ and several seconds, one often observes jumps around the typical conductance value for the molecule. Between these jumps, which can have an amplitude of an order of magnitude or more, one observes rapid fluctuations. The bandwidth of the experiment is usually limited to about $1 \mathrm{MHz}$ or less, so even the most rapidly observed fluctuations already represent an incoherent average over different junction configurations due to thermally accessible vibrations.

A widely adopted practice to deal with fluctuating observables is to study the fluctuation statistics,i.e., the conductance distribution taken over an ensemble of junctions realized in a series of experimental measurements. In practice, one repeatedly forms and breaks many junctions, records the digitized conductance during the contact-breaking process, and collects all data in a histogram, as illustrated in Fig. 3. It seems reasonable to expect that sufficiently deep indentation between recording traces restructures the metal leads and the molecular junction so that correlations between subsequent recordings are negligible. By combining the displacement length, measured from the point of metal-metal contact breaking, with the evolution of the conductance one can also build two-dimensional histograms (Martin, Ding, Sørensen et al., 2008), which are helpful for detecting multiple stable configurations and for obtaining a measure of the molecular bridge length.

The precise statistical properties of the ensembles generated in this way are hardly known and difficult to predict. At this stage an important simplification should arise because often 
the experimental recording cycles can be assumed to be extremely slow relative to the atomistic relaxation rates. Because of the resulting separation of timescales, one expects there to be enough time for the junction to relax into a set of particularly stable, "optimal" junction geometries. Presumably, at a slow-enough recording rate this set can be considered extremely small. This is the justification for the histogram technique to operate with concepts like "typical" junction geometries. It explains, in particular, why the corresponding atomistic shape used in theoretical simulations may possibly be derived from a variational principle rather than from a simulation of the junction geneses as they occur in the actual measurement. ${ }^{2}$ In the simplest case, the typical junction is identified as the most stable one, i.e., the one with the maximum binding energy. ${ }^{3}$ Adopting this logic, the peaks in the histogram are usually interpreted as representing energetically favorable junction configurations, and these are the most relevant parameters used for comparison with model calculations.

In the breaking process, the last-atom metal-to-metal contact is usually visible as a plateau near $1 G_{0}$, and this produces a sharp peak in the conductance histogram. Breaking of this last metal contact is followed by a jump out of contact (Agraiit, Rodrigo, and Vieira, 1993) to a conductance that is 1 or 2 orders of magnitude lower. In many cases, after this jump the current exponentially decreases with increasing separation of the electrodes, as expected for vacuum tunneling. Only for a fraction of the breaking events do one or more plateaus appear, signaling the successful bridging of the junction by a molecule. The large number of traces without a molecular signal results in a large background in the histograms. Initially, curves without a clear molecular signature were manually

\footnotetext{
${ }^{2}$ A further justification for the general practice may be found in the following argument. For the junction not to break in the presence of thermal fluctuations or bias-induced forces, there should be a notion of stability. This suggests that there is an optimization principle, which should become identical at zero bias with the optimization of the free energy under the boundary condition that the contact exists.

${ }^{3}$ To develop a statistical theory of the histogram technique would be rewarding but goes beyond the scope of this review. Two closely related issues should be briefly mentioned. (a) Molecular-dynamics (MD) investigations, e.g., those presented by French et al. (2013), have been put forward as an attempt to simulate the breaking of a molecular junction. For such studies simulating the long experimental timescales, which are associated with plastic deformation of the molecular junction under pulling, is challenging. Large system sizes and simulation times up to microseconds might be required. If these are affordable at all, then the approximations underlying the solution of the equations of motion required are extremely difficult to control. Examples of slow relaxation processes that should be properly described to be realistic include the temperature-driven diffusion of adatoms or multiatom exchange processes. Both processes can optimize or destabilize the junction geometry. (b) For similar reasons, the breaking of a molecular junction in the absence of a pulling force may also be ill described by MD simulations. This can happen in situations where breaking occurs due to rare, temperature-driven fluctuations. A careful discussion of the consequences of computational limitations for the interpretation of simulation results is not standard. For the reasons outlined earlier, the interpretation of MD-type studies for the statistical properties of molecular junctions needs to be done with precaution.
}

removed from the dataset. This practice has some risk of introducing experimenter bias in the data selection, and this practice has now been abandoned. The background problem can be reduced by the use of automated routines, for example, routines that detect the last step in the conductance (Jang et al., 2006). A widely adopted solution to the background problem is the use of histograms of the logarithm of conductance rather than the linear conductance (González et al., 2006). In this case, the background tunneling contribution reduces to a nearly constant contribution and the relevant features related to the molecule are more clearly visible in a dataset that now comprises all breaking traces.

$I(t)$ and $I(s)$ techniques. - The appearance of the shape of the histograms and the positions of the peaks for the same metal-molecule system do not reproduce perfectly between experimental groups, or even from one experimental run to the next. This implies that the underlying assumption that the repeated indentation effectively averages over all configurations is not fully justified. For example, one may anticipate that the results will be sensitive to parameters such as the voltage or current bias applied and the depth of indentation. This motivated Haiss et al. $(2003,2004)$ to avoid indenting the surface to maintain a common surface and tip structure. They developed the so-called $I(t)$ and $I(s)$ techniques. These techniques operate near room temperature and rely on bringing the STM tip close to the surface by the usual current feedback control. For low surface coverage, molecules with suitable anchoring groups are expected to jump stochastically into and out of contact with the tip. The difference between $I(s)$ and $I(t)$ is that the tip is moved in and out of close distance to the surface repeatedly for the former, while in the latter case the tip is held at a stable tunneling distance and the events are recorded as a function of time. The conductance values measured by $I(t)$ or $I(s)$ are typically found to be up to an order of magnitude smaller than the ones obtained from histograms produced by MCBJ or STM techniques.

\section{COMPUTATIONAL TECHNIQUES}

\section{A. A guided tour through quantum-transport theories}

The transport of charge, spin, and heat through a single molecule is a prime example of quantum transport through a mesoscopic device, where quantum coherence and correlations dominate the measured observables. For this reason, the standard mesoscopic transport technologies also apply in the case of single molecules.

An important line of research focuses on model studies, e.g., the single-impurity Anderson model, the Hubbard model, the Holstein model, etc.; for a recent review, see Thoss and Evers (2018). Models relevant for molecular transport are discussed in Sec. IV.

In contrast to most mesoscopic systems, single-molecule junctions consist of relatively few atoms, typically only a few hundred; moreover, their arrangement within the molecule is well known. This begs for $a b$ initio electronic-structure calculations. Concerning $a b$ initio transport computations, we identify three archetypical approaches as most prevalent:

(i) The NEGF (Kadanoff-Baym formalism) is a general approach. It applies to linear and nonlinear responses 
of interacting systems, in quasistatic and also timedependent situations. An additional attractive feature is that the coupling of electrons to vibrations is straightforward to implement (Pecchia and DiCarlo, 2004; Paulsson, Frederiksen, and Brandbyge, 2005; Paulsson et al., 2008).

This generality comes in situations where simplifications arise at the price of being somewhat inconvenient to use compared to competing methods. Meir and Wingreen (1992) worked out the most popular application of NEGF in mesoscopic transport. They derived explicit expressions for the $I-V$ curve that apply to generic quantum dots under the assumption of noninteracting electrodes.

(ii) When interested only in linear responses, the Kubo formula offers a viable alternative to NEGF. This formulation is advantageous because it involves only advanced and retarded Green's functions and therefore takes as an input only "equilibrium" (usually ground-state) electronic-structure information. Moreover, these Green's functions are available, at least in principle, already in standard electronic-structure codes. The reason is that advanced electronic-structure methods, such as the $G W$ theory, already operate with these objects. ${ }^{4}$

(iii) To the extent that interaction effects can be treated on a mean-field level, the Landauer-Büttiker formalism is efficient. It derives in a straightforward manner from NEGF [see Meir and Wingreen (1992)] and also applies to the nonlinear regime. This formulation underlies the standard $a b$ initio-based transport theory described later.

We emphasize that the list of methods mentioned here is not exhaustive. For example, a formalism based on the density-matrix theory as described in Bruus and Flensberg (2004) was also used with success (Donarini, Begemann, and Grifoni, 2010; Niklas et al., 2017).

\section{B. Brief overview of electronic-structure calculations for molecular junctions}

No matter which transport formalism is used, an input concerning the electronic structure of the device is needed. Indeed, molecular junctions pose one of the most difficult challenges of electronic-structure theory.

To see why this is so, we recall that even an isolated molecule requires advanced many-body techniques, e.g., for calculating ionization potentials (IPs) and electron affinities (EAs); see

\footnotetext{
${ }^{4}$ The $G W$ theory has been developed as a self-consistent leadingorder approximation that emerges from a diagrammatically exact representation of the many-body Green's function (Aryasetiawan and Gunnarsson, 1998; Aulbur, Jönsson, and Wilkins, 1999; Hedin, 1999; Bechstedt, 2015). Intuitively, it is understood that an improvement of Hartree-Fock theory is made by computing the Hartree potential with a screened interaction that is calculated on the level of the random phase approximation.
}

van Setten et al. (2015) for a review. This observation is relevant here because uncertainties in IPs (EAs) translate, in general, into errors in the position of transport resonances related to the highest occupied molecular orbital (HOMO) and lowest unoccupied molecular orbital (LUMO). Summarizing, estimates of IPs for small molecules based on Hückel studies or Kohn-Sham (KS) energies of DFT typically deviate from higher-level methods by $1 \mathrm{eV}$ or more (van Setten et al., 2015). ${ }^{5}$ For larger molecules or metallic wires, the absolute error in IPs sometimes decreases with the system size. This happens when the work function is dominated by a subsystem, such as a large metallic segment, for which the DFT functional applied is working well. This observation can be deceptive, however, because the most interesting molecular junctions display weakly connected subsystems ("molecular quantum dots") for which the errors in the computed level alignments remain large, even though the error in the overall work function could be relatively minor.

Therefore, one might have the impression that higherlevel methods, such as perturbative, Green's-function-based approaches $\left(G_{0} W_{0}\right)$, and wave-function-based methods (e.g., configuration-interaction methods and coupled-cluster theory), should provide the next generation standard tools of $a b$ initio transport calculations. However, there is an extra challenge, so the situation is not as clear. Despite its well documented shortcomings, molecular transport studies still mostly rely on KS-based scattering theory. The basic reason for the popularity of KS-based transport studies is that KS calculations, dealing essentially with a single-particle picture, digest large enough systems. Here "large enough" means that an approximation for the electronic structure can be found for the extended molecule, which comprises the molecule itself plus a part of the leads; see Fig. 4. ${ }^{6}$ Dealing with the extended molecule is important because transport phenomena are sensitive to how the molecular orbitals hybridize with the electrodes. This hybridization can be described consistently within KS simulations of extended molecules, but usually not so at an affordable cost with higher-level methods.

\section{Verification and validation of transport computations}

The geometry of a given molecular junction can be fluctuating in time driven, e.g., by thermal effects or the current flow. As we argue in Sec. II.D, the concept of a typical junction configuration should be well defined, nevertheless, for many experimentally relevant situations. Note that the

\footnotetext{
${ }^{5}$ In the case of KS theory, the IP can be calculated in two ways that are equivalent for exact DFT: One retrieves the IP either from the HOMO energy or from the difference in ground-state energies of the charged and charge-neutral molecular species [self-consistent field (SCF) method]. While the SCF method is known to give much more accurate results for the IP ("error cancellation"), it is the HOMO energy that actually enters the transport calculations.

"It would perhaps be preferable to speak of "molecule" versus "extended molecule" or "junction" versus "extended junction." The name extended molecule follows the established nomenclature, and we prefer to adhere to it, here. The word junction was chosen to indicate the part of the system that connects the metallic electrodes. This is not sharply defined, but it need not be because only the extended molecule plays a role in the calculations.
} 


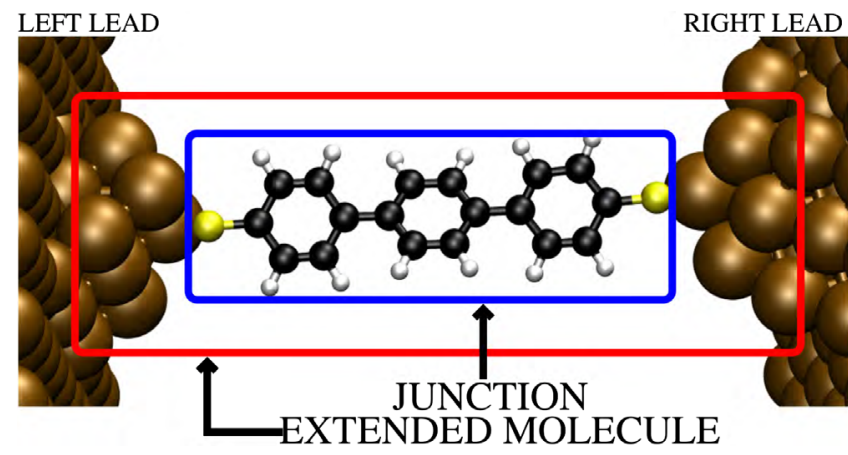

FIG. 4. Illustration of partitioning in model calculations: molecule, extended molecule (shown in red), semi-infinite leads. Adapted from Delle Site, 2018.

statement is not completely obvious, perhaps because many well investigated molecular junctions work with highly flexible molecules, such as alkanes, that do not by themselves (i.e., in the gas phase) provide a stable geometry.

The instance that the molecular geometry or the ensemble of geometries is not usually well known in experiments provides a major challenge for $a b$ initio simulations. Since in such computations the geometry usually is taken as given input, simulations mostly work with a plausible scenario for the geometry. Often they provide a consistent and plausible description, sometimes even quantitative, but scenarios are hardly ever microscopically validated by experiment.

It is rather straightforward to perform an internal consistency check on the simulation results: one determines to what extent the conclusions of the simulation are sensitive to variations of the geometry and the approximation level of the transport calculation; thus, a certain verification is possible. Nevertheless, the atomistic geometry remains a degree of uncertainty to keep in mind when comparing computations with experimental data. It superimposes the inherent theory uncertainty of electronic-structure calculations that results from parametrically uncontrolled approximations.

\section{The standard theory of $a b$ initio transport}

The standard theory of ab initio transport (STAIT) has been reviewed in several textbooks (Di Ventra, 2008; Haug and Jauho, 2008; Scheer and Cuevas, 2017). Efficient formulations of STAIT have been devised so that it can be implemented conveniently into many electronic-structure codes. The sheer number of implementations that have been reported over the years gives an impressive illustration of how important STAIT has become; an incomplete list includes McDCal (Taylor, Guo, and Wang, 2001), TranSIESTA (Brandbyge et al., 2002; Papior et al., 2017), SMEAGOL/Gollum (Rocha et al., 2006; Ferrer et al., 2014), two Turbomole-based codes (Pauly et al., 2008), and AITRANSS (Evers, Weigend, and Koentopp, 2004; Arnold, Weigend, and Evers, 2007), GPAW (Enkovaara et al., 2010), OpenMX (Ozaki, Nishio, and Kino, 2010), Atomistic NanoTransport (Jacob and Palacios, 2011), ASE (Larsen et al., 2017), and ATK (Smidstrup et al., 2019).

In the following, we briefly recapitulate STAIT while focusing on the conceptual underpinnings.

\section{Single-particle aspect, scattering theory, and partioning}

STAIT is a single-particle theory; it is effectively assumed that the many-body states of the molecular junction (at least in the low-energy sector) are reasonably well approximated by single Slater determinants. Equivalently, one assumes that the salient physics of the junction can be described in terms of an effective, single-particle Hamiltonian $H_{e \mathcal{M}}$ for the extended molecule. Now an almost universally met practice is to adopt the Kohn-Sham Hamiltonian $H_{\mathrm{KS}}$ for $H_{e \mathcal{M}}$.

For isolated molecules, the assumption that a single Slater determinant dominates is almost certainly doomed to fail because the interaction energy between valence electrons $U$ tends to exceed the typical level spacing. If the latter observation were to be true also for molecules within the junction, the phenomenon of the Coulomb blockade would preempt the domain of validity of STAIT.

However, the Coulomb interaction within the molecular junction is screened, reducing $U$ to a screened $U_{\text {scr }}$, so the overall situation can be complicated to analyze. As it turns out, there is a significant number of experimental situations where an effective single-particle theory provides a useful basis for data analysis. STAIT is the standard tool for evaluating what such a single-particle description would typically predict.

Depending on the emphasis, the transport formalism has been cast into different languages, including the NEGF (Di Ventra, 2008; Haug and Jauho, 2008; Stefanucci and Leeuwen, 2013; Scheer and Cuevas, 2017) and the LandauerBüttiker approach (Brandbyge et al., 2002; Evers, Weigend, and Koentopp, 2004). In either one, the current is expressed as

$$
I=\frac{e}{h} \int_{-\infty}^{\infty} d E \mathcal{T}(E)\left[f_{\mathcal{L}}(E)-f_{\mathcal{R}}(E)\right],
$$

where $f_{\mathcal{L}, \mathcal{R}}$ denote the Fermi distributions in the left and right contacts. The transmission function $\mathcal{T}(E)$ has the interpretation of a probability weight for a particle to be transmitted when it approaches the junction with energy close to $E$.

The most widely spread way for calculating $\mathcal{T}(E)$ is the partitioning approach. It distinguishes three regions, left lead ( $\mathcal{L}$, right lead $\mathcal{R}$, and the device region, that should be thought of as an extended molecule e $\mathcal{M}$; see Fig. 4. Thus, partitioning amounts to separating the Hilbert space of the full system into three sectors. In this formalism, one has

$$
\mathcal{T}(E)=\operatorname{Tr}\left[\Gamma_{\mathcal{L}}(E) G_{\mathrm{e} \mathcal{M}}(E) \Gamma_{\mathcal{R}}(E) G_{\mathrm{e} \mathcal{M}}^{\dagger}(E)\right],
$$

where the trace is to be taken over the device sector of the Hilbert space. Equation (2) has been derived first for noninteracting particles (Caroli et al., 1971); it remains valid at zero temperature also for systems with electron-electron interactions under the condition that the interaction with charge carriers in the leads (beyond mean field) can be neglected (Meir and Wingreen, 1992). When applied to electrons in the tunneling regime, Eq. (2) can be viewed as a generalization of Bardeen's theory of tunneling transport, going beyond the leading order in the tunneling amplitudes (Bardeen, 1961).

The advantage of partitioning becomes apparent in the definition of the Green's function that describes charge propagation on the extended molecule in the presence of the reservoirs, 


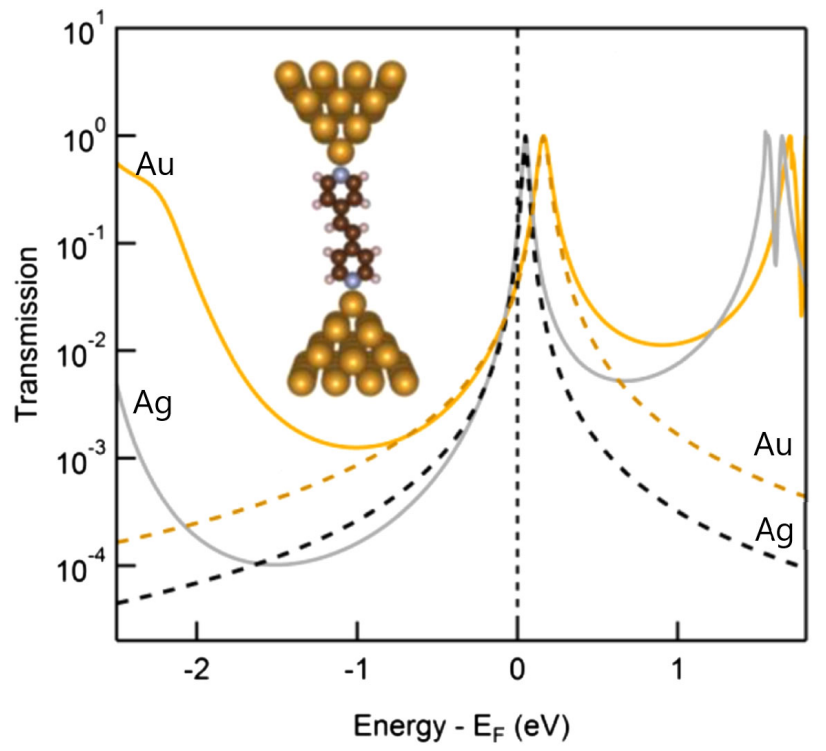

FIG. 5. Transmission function of 4,4'-vinylenedipyridine junction with $\mathrm{Au}$ and $\mathrm{Ag}$ electrodes (yellow and gray lines, respectively) calculated using STAIT. The vertical dashed line indicates the Fermi energy $\epsilon_{F}$. Dashed curves are Lorentzian fits. (Inse) The relaxed geometry with gold leads. From Adak et al., 2015.

$$
G_{e \mathcal{M}}(E)=\frac{1}{E-H_{e \mathcal{M}}-\Sigma_{\mathcal{L}}(E)-\Sigma_{\mathcal{R}}(E)} .
$$

It features a single-particle Hamiltonian $H_{e \mathcal{M}}$ that feeds into the transport formalism the electronic structure of the extended molecule, as it is provided by KS-based DFT calculations.

The matrices $\Gamma_{\mathcal{L}}, \Gamma_{\mathcal{R}}$ are electrode specific and do not carry information about the molecule; they denote the antiHermitian parts of the self-energies $\Sigma_{\mathcal{L}}, \Sigma_{\mathcal{R}}$ that describe the coupling of the extended molecule to the reservoirs $\Gamma_{\mathcal{L}}=\mathfrak{i}\left(\Sigma_{\mathcal{L}}-\Sigma_{\mathcal{L}}^{\dagger}\right)$, and similarly for $\mathcal{R}$. They can be calculated exactly, in principle, employing standard recursion methods (Groth et al., 2014; Walz, Bagrets, and Evers, 2015). Alternatively, simple approximative expressions can be used that become accurate when sufficiently many contact atoms are included in the extended molecule (Arnold, Weigend, and Evers, 2007).

A typical case.-In the most common scenario, $\mathcal{T}(E)$ shows a single peak near the Fermi energy of the reservoirs $\epsilon_{F}$ due to either the HOMO or the LUMO. As an example, we discuss now Fig. 5. At low temperatures, the LUMO is the only transport-active molecular orbital. The transmission peak is characterized by its position and width. Although the width is much smaller than the energy distance to the nearby levels, the shape of the peak is not Lorentzian in the tails due to quantum interference (QI). We elaborate on the QI effects in Secs. IV.A.1 and IV.B. The paradigm in Fig. 5 also shows that the conductance is strongly sensitive to the peak position, i.e., alignment of the LUMO with respect to $\epsilon_{F}$.

\section{Discussion of Kohn-Sham transport calculations}

A theoretical perspective on STAIT was given by Thoss and Evers (2018). We summarize the situation with a focus on
KS-transport calculations. The main issue for us is to what extent the KS Green's function $G_{\mathrm{KS}}$ can be a useful approximation to the real Green's function of the physical system.

(a) As is well known, in equilibrium, the KS Green's function $G_{\mathrm{KS}}=1 /\left(E-H_{\mathrm{KS}}-\Sigma_{\mathcal{L}}-\Sigma_{\mathcal{R}}\right)$ of the extended molecule relates to the local electron density $n(\mathbf{r})=2 \int_{-\infty}^{\epsilon_{F}} d E A_{\mathrm{KS}}(E, \mathbf{x})$ with a local spectral function $A_{\mathrm{KS}}(E, \mathbf{x})=-(1 / \pi) \mathfrak{s}\left\langle\mathbf{x}\left|G_{\mathrm{KS}}(E)\right| \mathbf{x}\right\rangle$. When employing exact exchange-correlation (XC) functionals, the KS Green's function reproduces the exact density $n(\mathbf{r})$. This does not imply that $A_{\mathrm{KS}}$ is also a good approximation to the physical spectral function $A(E, \mathbf{x})$; in general, it is not. For example, in the Coulomb-blockade regime the physical spectral function $A$ exhibits pronounced Hubbard sidebands, which are absent in $A_{\mathrm{KS}}$.

(b) The relation between $A_{\mathrm{KS}}(E)$ and the true spectral function $A(E)$ has been discussed since the 1980s, when band-structure calculations started using KS eigenvalues as approximations for quasiparticle energies (Perdew et al., 1982; Perdew and Levy, 1983; Sham and Schlüter, 1983; Yang, Cohen, and MoriSánchez, 2012). It is clear that there is no rigorous argument supporting this widespread practice; even with exact $\mathrm{XC}$ functionals, there is no known theorem guaranteeing that $G_{\mathrm{KS}}(E)$ will provide an accurate approximation for the exact Green's function $G(E)$.

Indeed, in the presence of strong Coulomb correlations, this is certainly not the case. As was pointed out by Burke, Köntopp, and Evers (2006), when evaluating the Kubo formula for noninteracting electrons with $G_{\mathrm{KS}}$ the resulting KS conductance reproduces the true conductance only up to a factor that accounts for an $\mathrm{XC}$ contribution to the voltage seen by KS particles.

(c) In the special case of well-separated transport resonances, there may be only a single transport-active level, HOMO* or LUMO*; see Fig. 5. In this situation, the single-impurity Anderson model (SIAM) applies; it features the Friedel sum rule, which allows one to express the conductance as a functional of the occupation of the frontier orbital $\mathcal{G}[n]$. Since the functional $\mathcal{G}[n]$ happens to be the same for interacting and noninteracting particles, the $\mathrm{KS}$ conductance can be quantitative, even though the spectral function is not physical (Stefanucci and Kurth, 2011; Bergfield et al., 2012; Tröster, Schmitteckert, and Evers, 2012). While the argument reproduced here is rigorous, it actually assumes symmetric coupling $\Gamma_{\mathcal{L}}=\Gamma_{\mathcal{R}}$. A generalization to the experimentally much more important case of asymmetric couplings has also been found (Evers and Schmitteckert, 2013). It hinges on the perhaps surprising observation that the specific ratio of rates $\Gamma_{\mathcal{L}} \Gamma_{\mathcal{R}} /\left(\Gamma_{\mathcal{R}}+\Gamma_{\mathcal{L}}\right)^{2}$ can be represented as a parameterfree density functional.

Summarizing, these considerations lead to an interesting situation: the conductance functional $\mathcal{G}[n]$ can reproduce the Kondo effect correctly in the 
transmission function [Eq. (2)] at the Fermi energy despite the KS Green's function $G_{\mathrm{KS}}$ failing to exhibit Abrikosov-Suhl resonance.

(d) While in many experimentally relevant cases the assumption of a single transport-active level may indeed apply, nevertheless, the corresponding KS conductance $G_{\mathrm{KS}}$ may not be quantitative. Two important factors intervene. First, the arguments employing Friedel's sum rule apply at temperatures below the Kondo temperature $T_{K}$ only. Experiments often are performed at elevated temperatures $T>T_{K}$, where the Coulomb blockade prevails. In this regime, the unphysical nature of $G_{\mathrm{KS}}$ renders the transport nearly resonant, while in reality the transmission is strongly suppressed (Stefanucci and Kurth, 2011). Second, explicit calculations operate with approximate XC functionals. As a consequence, the density profile $n(\mathbf{r})$ and, therefore, the input into $\mathcal{G}[n]$ are not sufficiently realistic for delivering quantitative conductances near $T=0$.

(e) In the majority of cases, the current is carried by more than one resonance, so the SIAM is not a fair description and extra quantum-interference effects can intervene. As a consequence, the connection between transport and Friedel's sum rule breaks down (Hackenbroich, 2001), and the protective mechanism that it provides for KStransport calculations presumably is not active. Hence, one is back to the lowest-order expectation based on Eq. (2), namely, that $G_{\mathrm{KS}}$ is limited in accuracy by the mismatch between $G_{\mathrm{KS}}$ and the exact Green's function. In other words, KS-transport calculations are only as good as the KS estimate of the electronic structure, which is embedded in $A_{\mathrm{KS}}(E, \mathbf{x})$.

\section{Proposed improvements over GGA-based Kohn-Sham calculations}

In the previous discussion, the principle applicability of KS theory for transport calculations was discussed. In practice, additional difficulties arise because actual computations always rely on approximate XC functionals, mostly local and semilocal ones, such as the local density approximation (LDA), generalized gradient approximations (GGAs) or the Perdew-Burke-Ernzerhof functional; for an overview of functionals, see Fiolhais, Nogueira, and Marques (2003). All these approximations neglect the "derivative discontinuity" (Perdew and Levy, 1983; Sham and Schlüter, 1983; Yang, Cohen, and Mori-Sánchez, 2012). This implies, roughly speaking, that Coulomb-blockade and related phenomena, e.g., partial charge transfer, are treated incorrectly, namely, on a meanfield level (Evers and Schmitteckert, 2013). There are numerous consequences that have been investigated over the past three decades in quantum chemistry and the computational materials sciences that we cannot cover here. For a first orientation, see e.g., Onida, Reining, and Rubio (2002) and Evers and Burke (2007). We briefly mention a few selected developments representative of the impact of the missing derivative discontinuity on ab initio transport simulations.

(a) Charge transfer can be a process that is critical for the properties of molecules on substrates, including their transmission properties. In their seminal work, Neaton, Hybertsen, and Louie (2006) developed an understanding of the relevant microscopic processes and analyzed to what extent they are captured by semilocal XC functionals.

(b) In KS theory, charge transfer is controlled by the alignment of energy levels of weakly coupled subsystems. Therefore, the charge-transfer problem goes along with an incorrect alignment of energy levels of weakly coupled subsystems. Ke, Baranger, and Yang (2007) investigated the consequences of incorrect level alignments for the transmission function.

(c) A problem with approximated $\mathrm{XC}$ functionals that derives from the fact that Hartree and exchange interactions are not being treated on the same footing is the so-called self-interaction error. Its impact on the conductance was discussed by Toher et al. (2005).

To improve upon the Green's functions $G_{\mathrm{GGA}}$ thus obtained, several procedures have been devised; an overview was given by Thoss and Evers (2018). Three main themes can be identified.

(i) One stays within the realm of KS theory, but one improves upon known artifacts of the GGA functionals. Specifically, optimized long-range separated functionals are introduced that provide a significantly better description of the partial charge transfer between molecule and substrate (Liu et al., 2017).

(ii) Alternatively, one leaves the realm of $\mathrm{KS}$ theory and computes a Green's function employing conventional many-body techniques, e.g., the $G_{0} W_{0}$ method (Bechstedt, 2015). Indeed, implementations of powerful $G_{0} W_{0}$ solvers for molecular matter are under way (Faber et al., 2014; Wilhelm and Hutter, 2016; Holzer and Klopper, 2017; Wilhelm et al., 2018). They open prospects for treating extended molecules with thousands of atoms and large enough basis sets so that controlled simulations can be performed with size-converged computational parameters (van Setten et al., 2015).

Early attempts in this direction were made by Thygesen and Rubio (2007) and Strange and Thygesen (2011). Because of computational limitations, the system sizes available at the time were not sufficiently large to demonstrate convergence with respect to the simulation volume. Therefore, the results were not fully conclusive. However, relevant fundamental questions were formulated that certainly need to be clarified in future research, for instance, concerning the importance of self-consistency (Thygesen and Rubio, 2008) and dynamical image-charge effects (Jin and Thygesen, 2014).

(iii) Rather than systematically computing a Green's function within a closed formalism [as in (ii)], one modifies the bare $G_{\mathrm{KS}}$ following a physically motivated recipe ("scissors operators" and "imagecharge corrections") (Quek et al., 2007; Mowbray, Jones, and Thygesen, 2008; Quek et al., 2009). The procedure carries a manifestly ad hoc character, 
and therefore its validity is difficult to evaluate systematically.

In this realm, a significant advancement was made in recent work by Celis Gil and Thijssen (2017). They determined the shift parameters for the scissors operators in a self-consistent procedure by computationally gating the molecule inside the junction and monitoring the evolution of charge with the gate voltage $Q\left(V_{g}\right)$. As is well known, approximate DFT functionals such as GGAs do not properly predict the shape of the charge evolution: as in typical meanfield approximations, $Q^{\mathrm{GGA}}\left(V_{g}\right)$ fails to exhibit a plateau at integer filling ("Coulomb blockade") in closed-shell calculations. Nevertheless, $Q^{\mathrm{GGA}}\left(V_{g}\right)$ is a useful object to study because the gate values that it takes to (de)populate the LUMO (HOMO) allow to reconstruct $U$, which is the key scissors parameter.

\section{Discussion of nonlinearities in the $I-V$ characteristics}

Generically, the $I-V$ characteristics exhibits a nonlinear shape that for many molecules is revealed on a scale well above $10 \mathrm{meV}$. As is seen in Eq. (1), nonlinearities can be due to the transmission function $\mathcal{T}(E)$ varying with energy $E{ }^{7}$ Because these terms are still linear in the difference of the Fermi functions $f_{\mathcal{L}}-f_{\mathcal{R}}$, we refer to them as nonlinearities of order zero.

Higher-order nonlinearities arise because the bias voltage $V_{b}$ can polarize the molecules and therefore affect the scattering potential, as illustrated in Fig. 6. Within the framework of STAIT such nonlinearities are conveniently included by allowing for a bias-voltage-dependent transmission function $\mathcal{T}\left(E, V_{b}\right)=\mathcal{T}_{0}(E)+\mathcal{T}_{1}(E) V_{b}+\cdots \quad$ in Eq. (1). The proper calculation of $\mathcal{T}\left(E, V_{b}\right)$ requires care. We include a corresponding discussion because it reveals, apart from technicalities, aspects of the basic mean-field-type physics of nonlinear $I-V$ characteristics.

Self-consistent calculations at finite bias.-Consider an extended molecule consisting of the molecule plus segments of left and right electrodes. In mean-field theories, the effective single-particle potential $v_{s}(\mathbf{r})$ that defines $H_{e \mathcal{M}}$ has to be constructed self-consistently from its eigenstates and eigenvalues. The calculation of the potential requires the density matrix $D\left(\mathbf{r}, \mathbf{r}^{\prime}\right)$ so that the potential can be expressed as a functional of the density matrix $v_{s}[D]$. In matrix notation (including spin) we can write

$$
D=\frac{-1}{\pi} \int_{-\infty}^{\infty} d E G_{e \mathcal{M}}\left(\Gamma_{\mathcal{R}} f_{\mathcal{L}}+\Gamma_{\mathcal{R}} f_{\mathcal{R}}\right) G_{e \mathcal{M}}^{\dagger},
$$

implying for the particle density $n(\mathbf{r})=D(\mathbf{r}, \mathbf{r})$. When focusing on zero-order nonlinearities, i.e., ignoring the feedback of the bias voltage on the transmission, one replaces the Fermi functions $f_{\mathcal{L}, \mathcal{R}}$ with the equilibrium distribution $f_{\text {eq }}$; this usually is also the first iteration step in a self-consistent

\footnotetext{
${ }^{7}$ In this section, we do not consider inelastic vibronic interactions. They also introduce nonlinearities in the $I-V$ curve, but these are not captured by $\mathcal{T}(E)$.
}

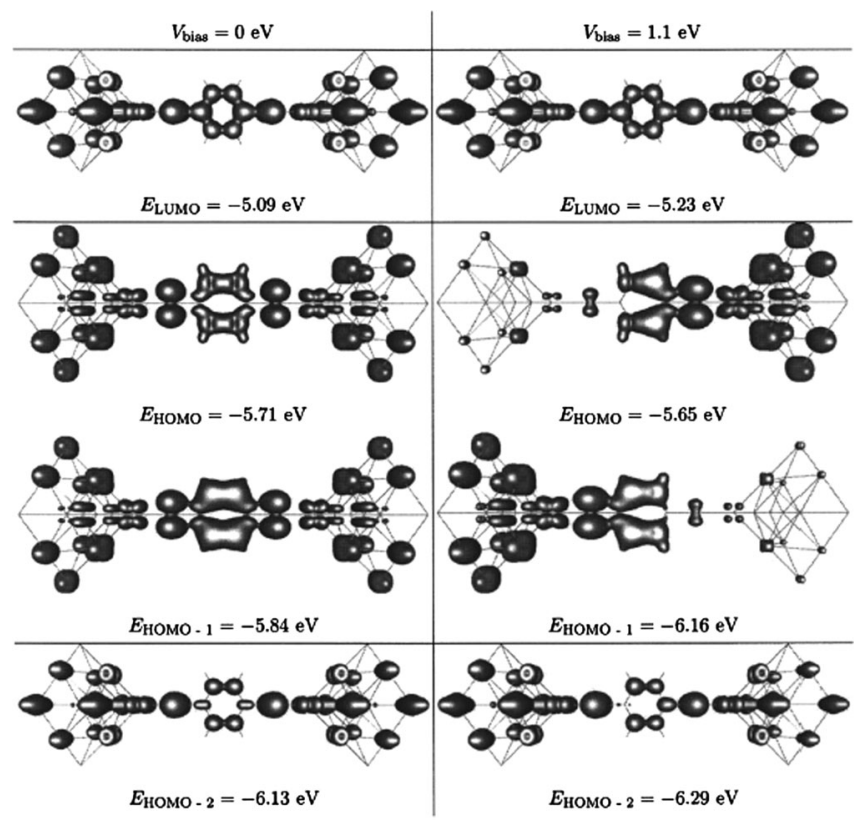

FIG. 6. Computational results for Au-benzenedithiol-Au junctions under high applied bias $V_{b}$. Atomic structure is indicated together with the electronic orbitals (density clouds) nearest $\epsilon_{F}$. At $V_{b}=1.1 \mathrm{~V}$ (right panels) the orbitals shift in energy, but they are also heavily distorted relative to $0 \mathrm{~V}$ (left panels). From Arnold, Weigend, and Evers, 2007.

nonequilibrium calculation. At the fixed point of the selfconsistency loop, the full form [Eq. (4)] is used for calculating $v_{s}[D]$ and the Hamiltonian $H_{e \mathcal{M}}$, respectively. As long as $V_{b}$ is not too large, one expects the fixed point to be unique.

Starting from equilibrium the self-consistent field cycle reshuffles electrons from one lead to the other, always keeping the net number of electrons of the extended molecule invariant (the charge-neutrality condition). ${ }^{8}$ At the fixed point an amount of charge $Q$ has been moved from one side to the other. For large enough electrodes taking the shape of a plate capacitor, $Q$ is proportional to the face area giving rise to a finite surface charge density $\sigma$. The bias-induced charge surplus feeds back into the single-particle energies of the electrode state and thus enters $D$. Thereby, the corresponding electric fields (surface dipole and capacitor field) are properly included in $v_{s}$ and thus become part of the mean-field solution (Arnold, Weigend, and Evers, 2007). Finally, the self-consistently calculated KS system yields the transmission function. The effect of the bias is shown in Fig. 7 for Au-BDT$\mathrm{Au}$, where BDT is benzenedithiol. At voltages $V_{b}<1 \mathrm{eV}$, the transport is dominated by the LUMO. The corresponding transmission resonance experiences a weak shift induced by the bias, and its real-space structure is largely unchanged; see Fig. 6. The effect of self-consistency on the $I-V$ characteristics is therefore weak at low bias. At bias $V_{b}>1 \mathrm{~V}$, the orbital pair HOMO and HOMO-1 plays an important role. These

\footnotetext{
${ }^{8}$ In practical terms, particle number $(N)$ conservation can be enforced within the iteration cycle in the following way: in each step one keeps the difference $\Delta \mu=\mu_{\mathcal{L}}-\mu_{\mathcal{R}}$ fixed but varies the average $\bar{\mu}=\left(\mu_{\mathcal{L}}+\mu_{\mathcal{R}}\right) / 2$ to conserve $N$ (Arnold, Weigend, and Evers, 2007).
} 

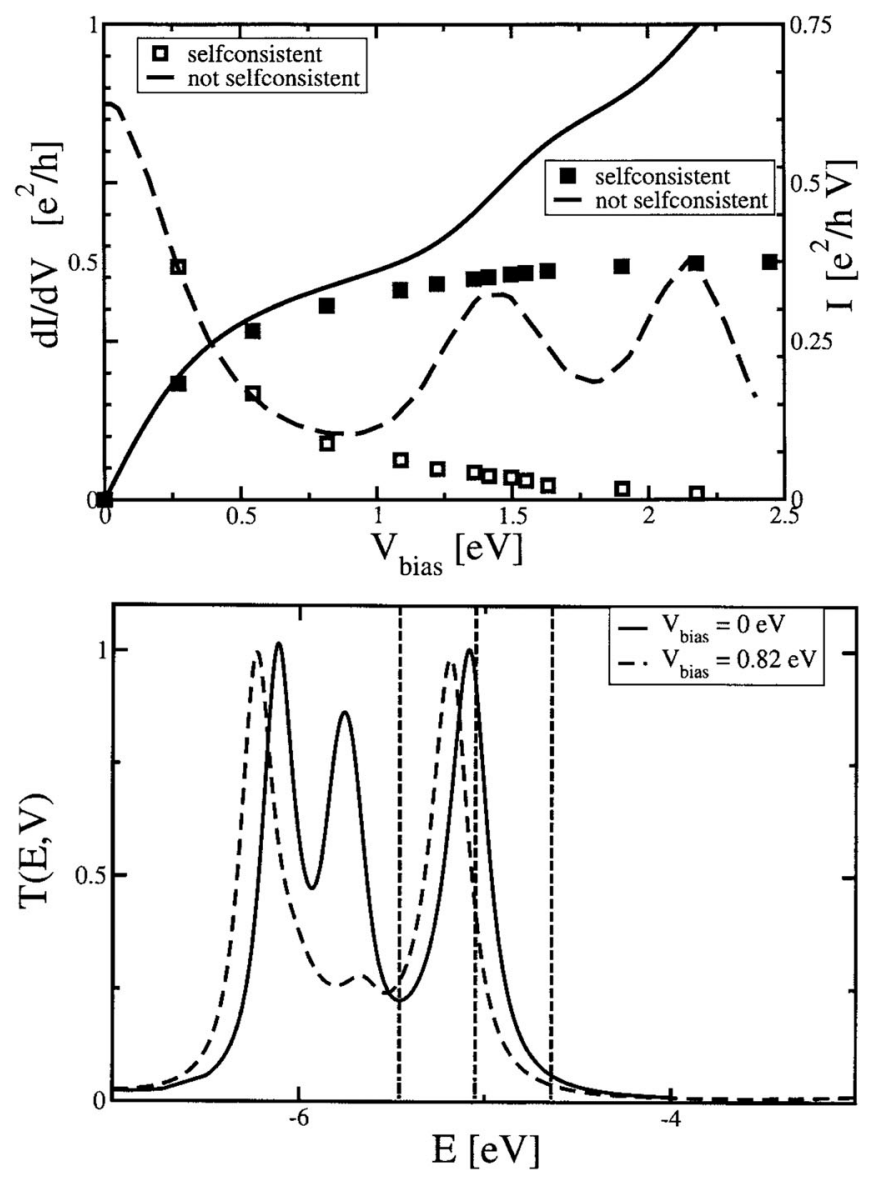

FIG. 7. Impact of self-consistency achieved under bias in transport computations for the Au-BDT-Au junction of Fig. 6. (Top panel) $I-V$ curve (right axis) and differential conductance $d I / d V$ (left axis). (Bottom panel) Comparison of the transmission at zero bias and at $V_{b}=0.82 \mathrm{~V}$. The vertical dashed lines are placed at $\epsilon_{F}-V_{b}, \epsilon_{F}, \epsilon_{F}+V_{b}$. The three peaks visible at zero bias correspond to LUMO, the pair HOMO and HOMO-1, and HOMO-2. The central peak is suppressed at the finite bias; see the text for an explanation. From Arnold, Weigend, and Evers, 2007.

nearly degenerate states mix strongly under the effect of bias, as shown by the wave functions in Fig. 6. The resulting states are each asymmetric, leading to suppression of the corresponding transmission resonances (around $-5.5 \mathrm{eV}$ in Fig. 7). This nonequilibrium Stark effect renders the molecular orbital pair "dark." The previously described mechanism leads to additional nonlinearity of the $I-V$ curve, suppressing the resulting current at higher bias.

Voltage drop.-At the fixed-point of the self-consistency iteration cycle, the orbitals of the leads (metal clusters) are shifted in energy away from their equilibrium position, upshifted in one electrode by $\mu_{\mathcal{L}}-\epsilon_{F}$, and down-shifted in the other by $\epsilon_{F}-\mu_{\mathcal{R}}$. The relative shift defines the bias voltage, $V_{b}$. As in experiments, $V_{b}$ can therefore also be "measured" in computational simulations by evaluating the relative energy shift in the raw data (Arnold, Weigend, and Evers, 2007).

We mention that even if the molecular junction exhibits an inversion or mirror symmetry along the axis of charge transport, the voltage drop cannot in general be expected to reflect this symmetric behavior as $\mu_{\mathcal{L}}-\epsilon_{F}=\epsilon_{F}-\mu_{\mathcal{R}}=(1 / 2) e V_{b}$.
Namely, the chemical potential of a lead, i.e., its work function, is sensitive to the surplus density $\sigma$ because the excess charge modifies the surface dipole. The detailed response depends on the atomistic structure of the electrode surface and is difficult to predict quantitatively, even with $a b$ initio calculations. Generally speaking, metal surfaces cannot be expected to exhibit a kind of particle-hole symmetry. Hence, one would not expect adding and subtracting charge to usually have the same quantitative effect (up to the sign) on the work function. ${ }^{9}$

Potential profile.-The profile of the voltage drop $\phi_{b}(\mathbf{r})$ can be read off at the self-consistent fixed point. It is essentially given by the contribution to the single-particle potential $\Delta^{Q} v_{s}(\mathbf{r})$ that arises due to the charge $Q$ being transferred within the self-consistency loop from one electrode to the other: $\Delta^{Q} v_{s}(\mathbf{r})=e \phi_{b}(\mathbf{r})$. In practical calculations the potential profile depends on the contact geometry, the shape of the electrode clusters, and, in particular, their size. Since the Coulomb interaction is long ranged, special care has to be taken with respect to the convergence of the transport simulation with system size; correspondingly, finitesize-converged computations can be demanding (Arnold, Weigend, and Evers, 2007).

Beyond zero-order nonlinearities.-We consider the Green's function of the real molecule $G_{\mathcal{M}}$ that emerges if we shrink the extended molecule by eliminating the metal clusters; it exhibits a structure analogous to Eq. (3). At the self-consistent fixed point, the molecular Hamiltonian $H_{\mathcal{M}}$ and the corresponding self-energies develop shifts away from their equilibrium values $\Delta H_{\mathcal{M}}=H_{\mathcal{M}}\left(V_{b}\right)-H_{\mathcal{M}}$. The biasinduced shift $\Delta H_{\mathcal{M}}$, in general, moves energy levels with respect to the electrode chemical potentials; it also deforms molecular wave functions so that the charge distribution on the molecule changes.

For example, as a consequence of the level shifts the molecule can charge or discharge. The dipole moment can also change due to the action of $\phi_{b}(\mathbf{r})$. It enters $\Delta H_{\mathcal{M}}$ as an external potential and summarizes the effects of the surface charges $\sigma$ accumulated on both electrodes. Under its action the molecule polarizes and a Stark shift of the molecular energy levels appears, both feeding into $\mathcal{T}_{1}(E)$.

Bias and current-induced forces.- Since the charge distribution in the molecular junction reacts to the applied bias, electrostatic forces should appear. The molecule will move under their action from its equilibrium position. This in turn modifies the molecular orbitals affecting higher-order nonlinearities in the $I-V$ and potentially also leads to switching behavior.

Such bias-induced forces exist even in the absence of a current flowing, and therefore should be distinguished from current-induced forces (Todorov, Hoekstra, and Sutton, 2001; Di Ventra, Pantelides, and Lang, 2002). While theoretical studies of the former are still scarce (Schnäbele, 2014), the latter have received considerable attention; see, e.g., Dundas, McEniry, and Todorov (2009), Bode et al. (2012), Todorov et al. (2014), and Lü et al. (2015) for examples. The physical

\footnotetext{
${ }^{9}$ In this respect, the case of $\mathrm{Au}$ could potentially be exceptional, because the bulk density of states is relatively flat near $E_{\mathrm{Fermi}}$.
} 
mechanisms behind current-induced forces are reciprocal: they are also felt by ion cores that move through an electronic bath. Therefore, the same mechanisms driving currentinduced forces also have implications for molecular-dynamics simulations; the corresponding generalized Langevin theory was reviewed by Lü et al. (2019). While experiments capable of resolving current-induced forces on the molecular scale are challenging, first indications of the effects have been reported (Sabater, Untiedt, and van Ruitenbeek, 2015).

The origin of current-induced forces was discussed in a particularly illuminating way by Lü, Brandbyge, and Hedegøard (2010). Our presentation is inspired by this work. Consider a kinetic equation for the vector $\mathbf{R}$ comprising the coordinates of all atoms measured with respect to their equilibrium positions. The equation takes the following form,

$$
M \ddot{\mathbf{R}}+\eta \dot{\mathbf{R}}+D \mathbf{R}=\mathbf{F}_{f}\left(V_{b}\right),
$$

where the usual assumptions underlying such kinetic equations are made. Most notably, a separation of timescales is assumed so that a Markovian ansatz is justified. The left-hand side (lhs) of Eq. (5) is merely the statement that the relaxation dynamics of $\mathbf{R}$ can be modeled by a collection of damped oscillators with mass tensor $M$. The matrix $D$ accounts for the restoring forces and is symmetric, reflecting Newton's third law. The matrix $\eta$ incorporates dissipation and also is symmetric, as can be seen, e.g., in the fluctuation-dissipation theorem. In addition, it is positive semidefinite to guarantee the second law of thermodynamics.

The right-hand side includes the fluctuating forces typical of Langevin-type descriptions. The equilibrium part of these forces $\mathbf{F}_{f}^{\mathrm{eq}}:=\mathbf{F}_{f}(0)$, is trendless by construction of $\eta$ and $D$. Out of equilibrium, for $V_{b} \neq 0$ trends exist that are naturally cast into a form analogous to the lhs of Eq. (5)

$$
\mathbf{F}_{f}\left(V_{b}\right)=\mathbf{F}_{f}^{\mathrm{eq}}+\mathcal{B} \dot{\mathbf{R}}+\mathcal{A} \mathbf{R}+\cdots .
$$

Formally, the matrices $\mathcal{A}$ and $\mathcal{B}$ can be decomposed into symmetric and antisymmetric constituents. Symmetric pieces, if they exist, combine with $\eta$ and $D$ and do not give rise to qualitatively new phenomena, at least at small enough $V_{b}$. Therefore, the symmetric pieces will be ignored and $\mathcal{A}$ and $\mathcal{B}$ are considered antisymmetric.

The matrix $\mathcal{A}$, being antisymmetric, cannot be understood as a second derivative of some energy functional with respect to a coordinate. It therefore represents a nonconservative force. Its effect on the dynamics is best illustrated by recalling that antisymmetry allows for rewriting the matrix-vector products appearing in Eq. (6) as vector products

$$
\mathbf{F}_{f}\left(V_{b}\right)=\mathbf{F}_{f}^{\mathrm{eq}}+\mathbf{B} \times \dot{\mathbf{R}}+\mathbf{A} \times \mathbf{R}+\cdots,
$$

where $\mathbf{B}=\left(-\mathcal{B}_{y z}, \mathcal{B}_{x z},-\mathcal{B}_{x y}\right)$, and analogously for $\mathbf{A}$. Hence, the third term of Eq. (7) represents a force that tends to rotate the direction of displacement $\mathbf{R}$. Since a rotation requires the definition of an axis to rotate about, the term arises because in nonequilibrium the currents flowing break isotropy. The effect of this term was observed by Dundas, McEniry, and Todorov (2009) as "water-wheel" forces.

The second term in Eq. (7) rotates the direction of the velocity $\dot{\mathbf{R}}$; it represents an effective "Lorentz force," where quotation marks remind us that the entries of $\mathbf{B}$ are matrix valued. Effective Lorentz forces are symmetry allowed since away from equilibrium with currents flowing time-reversal invariance and isotropy are broken. Since Lorentz forces are energy conserving, they actually allow for periodic orbits. In quantum models, such orbits are closely associated with geometric phases (also known as Berry phases). For the present context, Berry phases were discussed further by Lü, Brandbyge, and Hedegøard (2010).

The motion of the ion cores that results from the currentinduced forces feeds back into the electronic current. The effect was considered by Kershaw and Kosov (2017) by including corrections to the adiabatic response that are small in the ion velocities $\dot{\mathbf{R}}^{2}$. In extreme cases, the current-induced forces can lead to bond rupture. Progress toward a better understanding of this phenomenon was made in recent work by Erpenbeck et al. (2018).

\section{E. Transport viewed as relaxation and incoherent processes}

Thus far charge transport has been considered from the point of view of scattering theory. Here we slightly change our viewpoint and consider charge transmission as a relaxation problem. This alternative perspective allows for a relatively simple extension of the single-particle model that also includes inelastic effects. The extension presented here is qualitative; a more formal relation was worked out recently by Sowa et al. (2018).

\section{Alternative derivation of the trace formula}

We illustrate the strength of the relaxation perspective by using it to derive the key equation (1) in just a few lines. The transmission process is viewed as a decay of an electronic state of the left reservoir (source) into another one in the right reservoir (drain). This perspective is close in spirit to electron transfer theory, a connection that had been made before (Nitzan, 2001; Solomon, Andrews, Hansen et al., 2008). ${ }^{10}$

We now introduce our nomenclature. The wave functions of the left electrode with energy $\epsilon_{n}(k)$ are labeled by $|n, k\rangle$ for the incoming states and $|n,-k\rangle$ for the outgoing states, with $n$ denoting the channel index and $k>0$ the wave number. Similarly, for the right lead with energy $\epsilon_{n^{\prime}}\left(k^{\prime}\right)$ we use $\left|n^{\prime},-k^{\prime}\right\rangle$ for the incoming states and $\left|n^{\prime}, k^{\prime}\right\rangle$ for the outgoing states. The current flowing from the right to the left can then be written as

$I=\frac{e}{\hbar} \sum_{n, n^{\prime}} \iint d k d k^{\prime} \Gamma_{n^{\prime} n}\left(k^{\prime}, k\right)\left[f_{\mathcal{L}}\left(\epsilon_{n}(k)\right)-f_{\mathcal{R}}\left(\epsilon_{n^{\prime}}\left(k^{\prime}\right)\right)\right]$,

which is in the spirit of a rate equation: the current through the molecule is due to the decay of the states in the left lead that have energies $E$ within the voltage window. The associated

\footnotetext{
${ }^{10}$ One may also note that the structure of the equations to follow resembles those for Bardeen's theory of electron tunneling, as often applied for STM (Bardeen, 1961). However, there are several important differences. For example, in the Bardeen approximation the electronic structure of the states on the molecule is not taken into account, and states in the leads are assumed to remain unaffected by the formation of a junction.
} 
decay rate $\Gamma_{n^{\prime} n}\left(k^{\prime} k\right)$ has an exact representation in terms of the $\mathfrak{I}$ matrix (addressed later)

$$
\Gamma_{n^{\prime} n}\left(k^{\prime}, k\right)=2 \pi \delta\left(\epsilon_{n}(k)-\epsilon_{n^{\prime}}\left(k^{\prime}\right)\right)\left|\left\langle n^{\prime} k^{\prime}|\mathfrak{T}(E)| n k\right\rangle\right|^{2},
$$

which is readily understood as a generalization of Fermi's golden rule. Employing this relation and matching Eq. (8) to Eq. (1), we obtain for the transmission function

$$
\begin{aligned}
\mathcal{T}(E)= & (2 \pi)^{2} \sum_{n n^{\prime}} \iint d k d k^{\prime} \delta\left(E-\epsilon_{n}(k)\right) \delta\left(E-\epsilon_{n^{\prime}}\left(k^{\prime}\right)\right) \\
& \times\left|\left\langle n^{\prime} k^{\prime}|\mathfrak{T}(E)| n k\right\rangle\right|^{2} \\
= & 2 \pi \operatorname{Tr}_{\mathcal{R}} \delta\left(E-H_{\mathcal{R}}\right) \mathfrak{I}(E) \delta\left(E-H_{\mathcal{L}}\right) \mathfrak{I}^{\dagger}(E) .
\end{aligned}
$$

$H_{\mathcal{L}, \mathcal{R}}$ denote the Hamiltonians of the left and right leads and the trace is over the degrees of freedom of the right lead only. We arrive at Eqs. (1) and (2) by recalling that

$$
\mathfrak{I}(E)=v G_{e \mathcal{M}}(E) u^{\dagger}
$$

and defining $\Gamma_{\mathcal{L}}=2 \pi u \delta\left(E-H_{\mathcal{L}}\right) u^{\dagger} \quad$ and $\quad \Gamma_{\mathcal{R}}=2 \pi v \delta$ $\left(E-H_{\mathcal{R}}\right) v^{\dagger}$. The matrix $u(v)$ denotes the couplings of the extended molecule to the left (right) reservoir. The matrices connect states of the Hilbert space of the leads $\mathcal{R}$ and $\mathcal{L}$ to the Hilbert space of the extended molecule.

\section{Eigenchannel decomposition}

We comment on a misconception frequently met in connection with the trace formula

$$
\mathcal{T}(E)=\operatorname{Tr}\left[\Gamma_{\mathcal{L}} G_{e \mathcal{M}} \Gamma_{\mathcal{R}} G_{e \mathcal{M}}^{\dagger}\right] .
$$

The original version of the Landauer formula employs a representation of the transmission function

$$
\mathcal{T}(E)=\operatorname{Tr}_{\text {src }} \mathbf{t t}^{\dagger}
$$

where $\mathbf{t}$ denotes the matrix of transmission coefficients that describe the transfer of charge from a channel incoming from the source into a channel leaving into the drain (Imry, 2002). They constitute the off-diagonal elements of the scattering matrix and can be written as

$$
t_{n, n^{\prime}}=\frac{-2 \pi \dot{\mathfrak{i}}}{\sqrt{v_{n} v_{n^{\prime}}}}\left\langle n^{\prime} k^{\prime}|\mathfrak{T}(E)| n k\right\rangle
$$

where $v_{n}=d \epsilon_{n}(k) / d k$ and it is understood that $E=$ $\epsilon_{n}(k)=\epsilon_{n^{\prime}}\left(k^{\prime}\right)$. Correspondingly, the trace in Eq. (13) is to be taken over the transverse degrees of freedom ("channels") of the source as indicated by our nomenclature $\operatorname{Tr}_{\text {src }}$. The eigenvalues of $\mathbf{t t}^{\dagger}$ are the transmission coefficients, which are proper observables.

A tradition has been widely established that effectively identifies the object

$$
\tilde{\mathbf{t}}=\Gamma_{\mathcal{L}}{ }^{1 / 2} G_{\mathrm{e} \mathcal{M}} \Gamma_{\mathcal{R}}^{1 / 2}
$$

with t; see, e.g., Brandbyge et al. (2002) or Scheer and Cuevas (2017), Chap. 8.1. This identification is misleading because $\mathbf{t}$ and $\tilde{\mathbf{t}}$ are conceptually different: $\mathbf{t}$ carries indices that correspond to channel numbers, so acts on the transverse part of the Hilbert space of the leads. In contrast, $\tilde{\mathbf{t}}$ acts on the Hilbert space of the extended molecules. The former is physically uniquely defined, while the latter is subject to the partitioning scheme and is therefore of arbitrary size. This implies, in particular, that the number of eigenvalues of $\tilde{\mathbf{t}}^{\dagger}$ depends on the partitioning scheme, so these eigenvalues are not in general observables.

Despite the basic conceptual problem, eigenvalues of $\tilde{\mathbf{t}} \tilde{\mathbf{t}}^{\dagger}$ have been used successfully to interpret experiments, and one may wonder how this is possible. Presumably, the answer is that the dominating eigenvalues of $\tilde{\mathbf{t}} \tilde{\mathbf{t}}^{\dagger}$ approach the ones of $\mathbf{t t}^{\dagger}$ reasonably quickly once the Hilbert space of the extended molecule allows for enough transverse degrees of freedom. A careful analysis of the conditions of convergence has not yet been performed. In this context, we note that a decomposition of the transmission alternative to $\tilde{\mathbf{t}} \tilde{\mathbf{t}}^{\dagger}$ into a product of $q$ and $p$ matrices was investigated by Krstić, Zhang, and Buttler (2002).

\section{Limit of sequential transport and relation to the Marcus theory of charge transfer}

We now briefly turn to the strongly incoherent limit: the electron after flowing from an electrode onto the molecule dwells there for a very long time. By "very long" we mean that the electron loses all phase coherences due to its interactions with many molecular and environmental degrees of freedom. In this situation, transport can be considered sequential and the transmission probability takes a product form.

The source-drain picture of transport that we have embarked upon so far is closely related to the donor-acceptor concept familiar from electron transfer theory (Nitzan, 2006). The observation is useful because the latter theory suggests a phenomenological formulation of transport theory in the spirit of Marcus theory. The generalization captures incoherent and even inelastic aspects in the case of extremely weak coupling, where the dwell time of the charge carriers on the molecule is long enough for a segment of the molecule and/or its environment to restructure and thus destroy phase coherences. In this incoherent sequential limit, charge is transferred in a sequence of two hopping processes.

Along these lines concepts from electron transfer theory have been adopted for transport on molecular junctions (Nitzan, 2001). Recently applications to heat transfer across molecular interfaces and also to charge-transfer networks have been worked out (Craven and Nitzan, 2017a, 2017b). To the extent that conduction in the latter system class is diffusive, the connection to the macroscopic transport theories of material sciences, such as phonon-assisted hopping, has thus been made.

\section{MODEL-BASED ANALYTICAL RESULTS}

Models are an indispensable tool of understanding. In molecular transport, they serve to elucidate the physical principles involved for deriving explicit formulas, for estimating the relevant parameters, and for analyzing trends in the data. In addition, they are also needed to set up, analyze, interpret, and motivate further elaborate numerical computations. Therefore, in this section we give a brief overview of the models most relevant for understanding molecular junctions. 


\section{A. Qualitative discussion of few-level models}

In the vast majority of cases, only a few orbitals, typically only one or two, appear to be involved in molecular transport. These orbitals are usually weakly coupled in the sense that the contact mediated lifetime broadenings $\Gamma_{\mathcal{L}}, \Gamma_{\mathcal{R}}$ are much smaller than the relevant molecular energy scales, which would be, e.g., the HOMO-LUMO gap. In this situation, impurity models can provide a reliable description. Correspondingly, they are often employed for fitting and interpreting experimental data. We recapitulate the most basic facts.

\section{Two-level model without interactions}

A situation with only two transport-active orbitals is captured by a two-level model (TLM)

$$
\hat{H}=\sum_{\sigma=\uparrow, \downarrow} \sum_{i=0,1} \varepsilon_{i} \hat{n}_{i \sigma}
$$

as long as interactions can be ignored. The corresponding transmission function is straightforward to derive. The retarded resolvent operator takes the form $\hat{G}=[E-\hat{H}-\hat{\Sigma}(E)]^{-1}$, where as usual the self-energy facilitates the coupling to the reservoirs. Owing to the two-level structure and ignoring the spin, the resolvent can be represented by a $2 \times 2$ matrix $G(E)$, whose explicit structure depends on the choice of the basis. The corresponding matrix elements define the Green's function. Irrespective of the basis choice, a "rotation" $Q$ can be found such that $G(E)$ takes the form

$$
G(E)=Q\left(\begin{array}{cc}
E-z_{0} & 0 \\
0 & E-z_{1}
\end{array}\right)^{-1} Q^{-1},
$$

with $z_{i}=\varepsilon_{i}+\Sigma_{i}$ and $i=0,1$; here $\Sigma_{i}(E)$ is denoting the leadinduced shift of the pole positions into the complex plane. The columns of $Q, \psi_{i}^{r}$, are given by the solutions of the eigenvalue problem

$$
[\hat{H}+\hat{\Sigma}(E)] \psi_{i}^{r}(E)=z_{i}(E) \psi_{i}^{r}(E), \quad i=0,1,
$$

while the rows of $Q^{-1}, \psi_{i}^{1 *}$, solve

$$
\psi_{i}^{1 *}(E)[\hat{H}+\hat{\Sigma}(E)]=z_{i}(E) \psi_{i}^{1 *}(E)
$$

For a detailed mathematical discussion, see Farid (1999).

Motivated by the trace formula (2), we introduce the abbreviations $\tilde{\Gamma}_{\mathcal{L}}=Q^{\dagger} \Gamma_{\mathcal{L}} Q$ and $\tilde{\Gamma}_{\mathcal{R}}=Q^{-1} \Gamma_{\mathcal{R}} Q^{\dagger-1}$. Then, employing Eq. (2), the transmission can be written as a sum of three terms

$$
\mathcal{T}_{\mathrm{TLM}}(E)=\mathcal{T}_{0}+\mathcal{T}_{1}+\mathcal{T}_{01}
$$

with two direct terms $i=0,1$ and an interference term

$$
\mathcal{T}_{i}=2 \frac{\tilde{\Gamma}_{\mathcal{L} i i} \tilde{\Gamma}_{\mathcal{R} i i}}{\left|E-z_{i}\right|^{2}}, \quad \mathcal{T}_{01}=\Re \frac{4 \tilde{\Gamma}_{\mathcal{L} 01} \tilde{\Gamma}_{\mathcal{R} 10}}{\left(E-z_{1}\right)\left(E-z_{0}\right)^{*}} .
$$

In these exact expressions, the pole positions as well as the residues are functions of energy $E$. If we assume that the energy variation of the self-energy $\Sigma(E)$ due to coupling with the leads is sufficiently weak, a simple two-pole structure is recovered:

$$
\mathcal{T}_{\mathrm{TLM}}(E)=2\left|\frac{\sqrt{\Gamma_{\mathcal{L} 0} \Gamma_{\mathcal{R} 0}}}{E-z_{0}}+e^{i \Psi_{c}} \frac{\sqrt{\Gamma_{\mathcal{L} 1} \Gamma_{\mathcal{R} 1}}}{E-z_{1}}\right|^{2} .
$$

Here $\Gamma_{\mathcal{L} i}, \Gamma_{\mathcal{R} i}$ denote (twice) the imaginary parts of $\Sigma_{i}$ taken at the pole positions and resolved per left or right lead contribution. The overall prefactor of two accounts for the spin and the phase factor $e^{i \Psi_{c}(E)}$ parametrizes interference effects (Géranton et al., 2013).

Experimental $I-V$ traces of molecular junctions can often be modeled, phenomenologically, in terms of formulas like Eq. (19). We stress that this observation does not necessarily imply that the corresponding fitting parameters have meaningful interpretations in terms of a picture of noninteracting particles. As we point out in Sec. IV, a two-pole structure in the Green's function can also arise as a consequence of strong Coulomb interactions. In this case, fitting a two-resonance transmission similar to Eq. (19) can be successful, while the interpretation of the resulting fitting parameters is fundamentally different.

Equation (19) provides the basic concepts for discussion of quantum interference, a topic that is elaborated on in Sec. IV.B. To make contact to the conventional representation of a Fano line shape, ${ }^{11}$ we introduce two real-valued, dimensionless parameters

$$
\begin{gathered}
A=4 \Im\left[\frac{\tilde{\Gamma}_{\mathcal{R} 01} \tilde{\Gamma}_{\mathcal{L} 10}}{\left(E-z_{0}\right) \Gamma_{1}}\right]+4 \frac{\tilde{\Gamma}_{\mathcal{R} 11} \tilde{\Gamma}_{\mathcal{L} 11}}{\Gamma_{1}^{2}}, \\
B=4 \Re\left[\frac{\tilde{\Gamma}_{\mathcal{R} 01} \tilde{\Gamma}_{\mathcal{L} 10}}{\Gamma_{1}\left(E-z_{0}\right)}\right],
\end{gathered}
$$

and a dimensionless energy $\epsilon=\left(E-E_{1}\right) /\left(\Gamma_{1} / 2\right)$ defined by the real and imaginary parts of the pole $z_{1}=E_{1}+\mathfrak{i} \Gamma_{1} / 2$; we thus obtain

$$
\mathcal{T}_{\mathrm{TLM}}(E)=2\left[\frac{\tilde{\Gamma}_{\mathcal{L} 00} \tilde{\Gamma}_{\mathcal{R} 00}}{\left|E-z_{0}\right|^{2}}+\frac{A+B \epsilon}{1+\epsilon^{2}}\right] .
$$

The second term in Eq. (22) displays the typical Fano shape under the assumption that $\Gamma_{1} / 2$ sets the smallest energy scale, so $E_{1}, A$, and $B$ are approximately constant on this scale. Then $\Gamma_{1}$ defines the width of the asymmetric line shape, the sign of $A$ determines its resonant versus antiresonant character, and $B$ controls the degree of asymmetry. Under the assumptions made here, the first term in Eq. (22) is weakly varying on the resonance scale $\Gamma_{1}$; correspondingly, it plays the role of a background transmission.

We mention that in the artificial case in which the three matrices $G(E)$ and $\Gamma_{\mathcal{L}, \mathcal{R}}$ commute, we have $B=0$ and $\mathcal{T}_{\text {TLM }}(E)$ decomposes into independent resonances with two orthogonal transmission channels (i.e., without quantum interference).

\footnotetext{
${ }^{11}$ We use the term Fano resonance in a loose sense. Traditionally, it refers to the scattering of a free particle (continuous spectrum) off a potential with a bound state (discrete spectrum) and includes a twopath QI contribution (Fano, 1961). We include cases where the dominating interfering paths all run through bound molecular orbitals (e.g., through HOMO and LUMO). Our motivation is that the characteristic line shape, Eq. (22), does not distinguish both situations, emphasizing that they are conceptually the same.
} 


\section{Basics of SIAM, Coulomb blockade, and Kondo effect}

The SIAM considers a single transport-active orbital with interaction $U$ :

$$
\begin{aligned}
\hat{H} & =\hat{H}_{\mathcal{M}}+\hat{H}_{\mathcal{T}}+\hat{H}_{\mathcal{X}}, \\
\hat{H}_{\mathcal{X}} & =\sum_{k} \sum_{\sigma} \varepsilon_{k} \hat{c}_{k \sigma}^{\dagger} \hat{c}_{k \sigma}, \\
\hat{H}_{\mathcal{T}} & =\sum_{k} \sum_{\sigma} V_{k} \hat{c}_{k \sigma}^{\dagger} \hat{d}_{\sigma}+\text { H.c., } \\
\hat{H}_{\mathcal{M}} & =\sum_{\sigma} \varepsilon_{0} \hat{n}_{0 \sigma}+U \hat{n}_{0 \uparrow} \hat{n}_{0 \downarrow} .
\end{aligned}
$$

It has proven useful in diverse physical contexts, such as transition-metal impurities in metals or semiconductor quantum dots (Kouwenhoven and Glazman, 2001). Similar to STAIT, the SIAM employs partitioning featuring the canonical creation and annihilation operators of the electrons in the leads $\hat{c}_{k \sigma}, \hat{c}_{k \sigma}^{\dagger}$ and of the molecular "quantum dot" $\hat{d}_{\sigma}, \hat{d}_{\sigma}^{\dagger}$. The molecule is represented by the dot Hamiltonian $\hat{H}_{\mathcal{M}}$ : the on-site energy $\varepsilon_{0}$ is defined with respect to the chemical potential in the leads, $\hat{n}_{0 \sigma}=\hat{d}_{\sigma}^{\dagger} \hat{d}_{\sigma}$ denotes the number operator for electrons occupying the single level with either spin up or down $\sigma=\uparrow, \downarrow$, and $U$ represents the charging energy of the level. The Hamiltonian $\hat{H}_{\mathcal{X}}$ implements the left and right reservoirs for $\mathcal{X}=\mathcal{L}, \mathcal{R}$. For compactness of notation, we denote all degrees of freedom of the electrons in the reservoir by $k$ and $\sigma$ ( $k$ encodes the band index, lead index, wave numbers, etc.). Finally, $\hat{H}_{\mathcal{T}}$ is the tunneling Hamiltonian, with the matrix elements $V_{k}$ corresponding to the matrices $u$ and $v$ in Eq. (12).

Coulomb blockade.- In the limit $U=0$, the SIAM reduces to a single noninteracting resonant level with the width $\Gamma=\Gamma_{\mathcal{L}}+\Gamma_{\mathcal{R}}=2 \pi \sum_{k}\left|V_{k}\right|^{2} \delta\left(\varepsilon_{k}\right)$. For the other limit $U \neq 0$ and small $\Gamma$, the level is decoupled from the leads and the molecular Hilbert space comprises four states, which describe the empty, the singly occupied (spin up or spin down), or the doubly occupied level. The salient point of the SIAM is that it can describe a configuration with partially filled orbitals ("open shells") and an integer filling $N_{0}=\sum_{\sigma}\left\langle\hat{n}_{0 \sigma}\right\rangle \approx 1$ that does not break spin-rotational invariance, i.e., does not display magnetism. This defines the regime of the Coulomb blockade that prevails at nonvanishing $\Gamma$ and at large enough charging energy $U$ (Anderson, 1961) such that ${ }^{12}$

$\varepsilon_{0}<0<\varepsilon_{0}+U, \quad \Gamma /\left|\varepsilon_{0}\right| \ll 1, \quad \Gamma /\left|\varepsilon_{0}+U\right| \ll 1$.

The retarded Green's function of the isolated level is a $2 \times 2$ matrix in spin space; it is spin diagonal with entries

\footnotetext{
${ }^{12}$ We reiterate that with noninteracting particles orbital fillings are either 0 or 2, except when the orbital energy $\varepsilon_{0}$ is resonant with the chemical potential: $\left|\varepsilon_{0}-\epsilon_{F}\right| \lesssim \Gamma$. At large $U$, the filling can be odd integer in a much larger regime $\epsilon_{F}-\varepsilon_{0} \lesssim U$. Mean-field theories such as the Hartree-Fock method can also realize odd-integer fillings, but at the price of invoking magnetism, i.e., by spontaneously breaking spin-rotational invariance. This mean-field artifact is known as the symmetry dilemma.
}

$$
G_{0, \uparrow}(E)=\frac{1-\left\langle\hat{n}_{0 \downarrow}\right\rangle}{E-\varepsilon_{0}+\mathfrak{i} 0}+\frac{\left\langle\hat{n}_{0 \downarrow}\right\rangle}{E-\varepsilon_{0}-U+\mathfrak{i} 0},
$$

and similar for the spin-down component in the limit $\Gamma=0^{+}$ (Bruus and Flensberg, 2004; Haug and Jauho, 2008). Introducing the lead coupling, the two peaks of the $G_{0, \uparrow}(E)$ shift and acquire some broadening. In addition, a third peak emerges at the Fermi energy, the Abrikosov-Suhl (also Kondo) resonance, which signals the onset of the Kondo effect.

Kondo effect.- In the regime given by the conditions (24), the electronic spin of the molecule represents a degenerate two-level system. The associated quantum fluctuations become increasingly important as the temperature is lowered, leading to the Kondo effect. A manifestation of the latter is the screening of the local magnetic moment of the molecule that goes together with the emergence of the Kondo resonance. The characteristic energy scale is (Hewson, 1993)

$$
k_{B} T_{K}=E_{0} \exp \left[-\frac{\pi}{\Gamma}\left(\frac{1}{\varepsilon_{0}+U}-\frac{1}{\varepsilon_{0}}\right)^{-1}\right],
$$

where the factor $E_{0} \simeq \max (D, U)$ and $D$ denotes the halfwidth of the lead's conduction band. ${ }^{13}$

Since the Kondo temperature $T_{K}$ is exponentially small in the ratio $U / \Gamma$, the Kondo effect is not commonly observed in molecular junctions, where the coupling $\Gamma$ is typically small compared to the charging energy $U$. Focusing on temperatures above $T_{K}$, where we can ignore the Kondo peak, we arrive at the following simplified representation:

$$
G_{\uparrow}=\frac{1-\left\langle\hat{n}_{0 \downarrow}\right\rangle}{E-\varepsilon_{0}-\Sigma}+\frac{\left\langle\hat{n}_{0 \downarrow}\right\rangle}{E-\varepsilon_{0}-U-\Sigma}
$$

(Haug and Jauho, 2008), with $\Sigma(E)=\Sigma_{\mathcal{L}}(E)+\Sigma_{\mathcal{R}}(E)$. It features only two resonances, the upper and lower Hubbard peaks, for a half-filled level $\left\langle\hat{n}_{0, \uparrow}\right\rangle=\left\langle\hat{n}_{0, \downarrow}\right\rangle=1 / 2$. In more realistic theoretical treatments (still keeping $T \gg T_{K}$ ), the width of the peaks can differ (Pruschke and Grewe, 1989; Könemann et al., 2006).

Discussion of transmission and interference features.After feeding Eq. (27) into the trace formula (2), we obtain

$\mathcal{T}_{\text {SIAM }}(E)=2 \Gamma_{\mathcal{L}} \Gamma_{\mathcal{R}}\left|\frac{1-\left\langle\hat{n}_{0 \downarrow}\right\rangle}{E-\varepsilon_{0}-\Sigma}+\frac{\left\langle\hat{n}_{0 \downarrow}\right\rangle}{E-\varepsilon_{0}-U-\Sigma}\right|^{2}$.

Because of the charging effects, two Hubbard-peaks emerge that are well separated from each other under the conditions (24). While the transmission functions, Eqs. (19) and (28), describe two different physical situations-with and without strong correlations - they share the same analytical structure. Therefore, when interpreting measurements by fitting a single-particle theory such as STAIT, Eq. (19) can be misleading. The difficulty exists, in particular, in situations where the $a b$ initio theory for the ground state predicts halffilled levels.

In metallic or semiconducting quantum dots, one usually encounters a spacing of single-particle levels that is much

\footnotetext{
${ }^{13}$ Since $T_{K}$ is the temperature of a crossover, some ambiguity exists in the definition of $E_{0}$. In any case, the exponential implies a strong suppression of $T_{K}$ in the limits (24).
} 

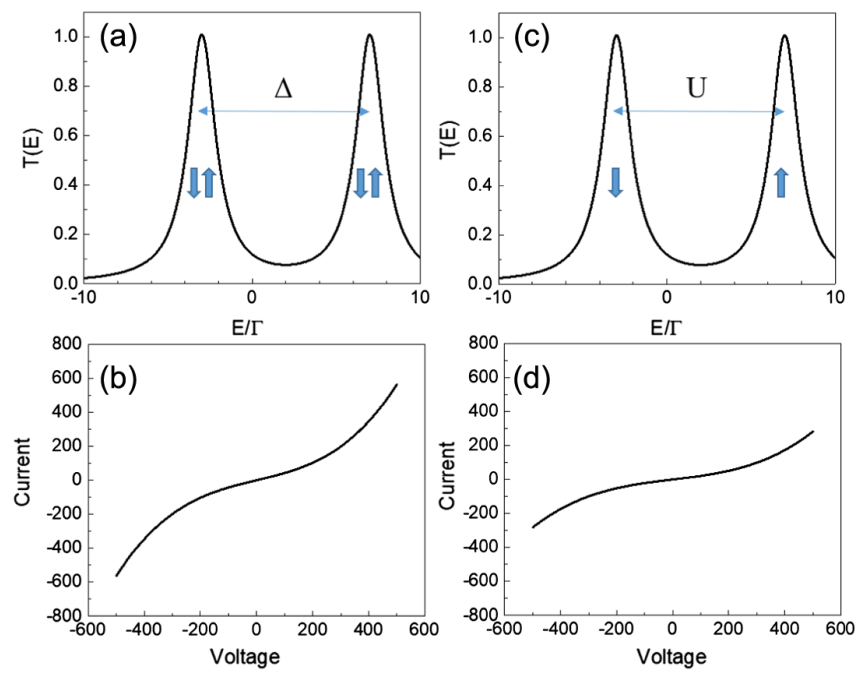

FIG. 8. Schematic illustration of the similarity between two models at two extreme limits. (a) When the single-particle level separation $\Delta=\varepsilon_{1}-\varepsilon_{0}$ and the level broadening $\Gamma$ are much larger than the on-site Coulomb repulsion $U$, we may neglect the latter. The transmission has two spin degenerate resonances, and the $I-V$ characteristics (b) are expected to show a generic $S$ shape determined predominantly by the nearest level, in this example the HOMO. (c) In the other limit, when $U \gg \Gamma$ we again obtain two resonances, but here they are single-spin resonances separated by $U$. However, when measuring an $I-V$ curve the shape (d) will be indistinguishable from the noninteracting two-level model, although the amplitudes may differ. Here we assume that $T_{K} \ll T$.

smaller than the interaction strength $\left|\varepsilon_{1}-\varepsilon_{0}\right| \ll U$. Such a clear separation of energy scales is less common in molecular junctions, where the HOMO-LUMO gap is typically of the order of $U$. This often makes it challenging to clearly identify correlation effects in molecular junctions from the measured $I-V$ characteristics alone, as illustrated in Fig. 8.

Remark on Fano line shapes in the Kondo regime.--In the Kondo regime, the elementary excitations are of Fermi-liquid type (Nozières, 1974). Correspondingly, the Kondo resonance is simply understood as an extra pole in the Green's function

$$
G_{\sigma}(E) \approx \frac{Z_{\mathrm{K}}}{E+\mathfrak{i} E_{\mathrm{K}}}+\tilde{g}
$$

which naturally appears at zero energy; its width is given by $E_{K} \approx k_{B} T_{K}$. The smooth background $\tilde{g}$ is small compared to the resonant term.

By virtue of the Fermi-liquid nature of the Kondo ground state, Eq. (29) can be used in combination with Eq. (2); the resulting transmission has the structure of a single resonant level (neglecting the small $\tilde{g}$ ). The residue $Z_{K} \leq E_{K} / \Gamma$ guarantees that the maximum conductance is limited to $1 G_{0}$.

When another transport orbital is present or when there is direct lead-to-lead tunneling, this can be represented by a background scattering amplitude $\tilde{t}$, and the $\mathfrak{T}$ matrix (12) can be written in the form

$$
\mathfrak{I}(E)=v G_{\sigma}(E) u^{\dagger}+\tilde{t}(E)
$$

Typically, the energy dependence of $\tilde{t}(E)$ is smooth on the scale of $E_{K}$, and the transmission probability attains the same structure as for quantum interference, Eq. (22), with a Fano line shape (Újsághy et al., 2000; Plihal and Gadzuk, 2001).

Kondo blockade.-Mitchell et al. (2017) made an interesting prediction that the Kondo effect can lead to a suppression of the conductance, termed the Kondo blockade. The crucial condition for its realization is that the molecular spin is coupled to two independent conduction channels. The conductance is suppressed because of an intricate combination of many-body effects that may be viewed as a specific hallmark of Kondo physics in molecular systems.

Let us consider a molecule with a spin-half ground state coupled to a pair of reservoirs. Because of spin-rotational invariance, the low-energy description has the form of a Kondo Hamiltonian (Nozières and Blandin, 1980),

$\hat{H}_{2 \mathrm{CK}}=\sum_{\mathbf{k}} \sum_{\alpha \underline{\alpha \sigma} \underline{\sigma}}\left(\frac{1}{2} J_{\alpha \underline{\alpha}} \hat{\mathbf{S}} \cdot \boldsymbol{\tau}_{\sigma \underline{\sigma}}+W_{\alpha \underline{\alpha}} \delta_{\sigma \underline{\sigma}}\right) \hat{c}_{\alpha \sigma}^{\dagger}(\mathbf{k}) \hat{c}_{\underline{\alpha} \underline{\sigma}}(\mathbf{k})$,

where $\alpha, \underline{\alpha}$ each sum over the states in the right and left leads $\alpha, \alpha \in\{\mathcal{L}, R\} ; \hat{\mathbf{S}}$ is the spin operator of the molecule and $\boldsymbol{\tau}$ are Pauli matrices, with $\sigma$ and $\underline{\sigma}$ summing over both spin directions.

The second term in Eq. (31) represents a description of the molecule in terms of a scattering potential $W$. The first term has the form of exchange; it is the one that gives rise to spinflip scattering, which ultimately leads to the Kondo effect. In the presence of two electrodes, the coupling $J$ and $W$ take the form of $2 \times 2$ matrices. The conventional treatment proceeds with a rotation of the basis, so $J$ is brought into a diagonal form. Thus, two superpositions of electrode states arise, $\hat{\psi}_{e, o}$, that are referred to as even and odd channels.

It turns out that, because of its single-level nature, the coupling $J$ has a zero eigenvalue in the case of the SIAM, $J_{o}=0$ (Glazman and Raikh, 1988). In more generic situations, both eigenvalues are nonvanishing. If $J$ and $W$ can be simultaneously diagonalized ( $J$ and $W$ commute), then we arrive at the conventional two-channel Kondo model (Nozières and Blandin, 1980). With molecules, this is not typically the case, however. Several new energy scales enter with the implication that the conductance is no longer universal (i.e., a function of $T / T_{K}$ only). One of the new phenomena arising is possible interference between the first and the second channel that is mediated via the potentialscattering term. As Mitchell et al. (2017) showed, this interference phenomenon can lead to a suppression of conductance, which is the essence of the Kondo blockade.

\section{Two-impurity Anderson model}

In this section, we discuss correlation effects involving a second transport-active molecular orbital, as it arises, e.g., in open $d$ shells of transition-metal complexes or organic polyradicals. Qualitatively new phenomena occur, e.g., the underscreened Kondo effect. It was predicted for impurity systems by Nozières and Blandin (1980) and has finally been observed for the first time in a molecular junction (Roch et al., 2009).

A general way to include two transport-active orbitals is the two-impurity Anderson model (TIAM) (Alexander and 
Anderson, 1964). It reveals a rich phenomenology. We review the corresponding experiments that have been performed in the context of molecular junctions in Sec. V. In this section, we provide a theoretical background that will be helpful for the interpretation of these experiments.

In full analogy with the SIAM, the TIAM Hamiltonian reads

$$
\begin{gathered}
\hat{H}=\hat{H}_{\mathcal{M}}+\hat{H}_{\mathcal{T}}+\hat{H}_{\mathcal{X}}, \\
\hat{H}_{\mathcal{X}}=\sum_{k} \sum_{\sigma=\uparrow, \downarrow} \varepsilon_{k} \hat{c}_{k \sigma}^{\dagger} \hat{c}_{k \sigma}, \\
\hat{H}_{\mathcal{T}}=\sum_{k} \sum_{\sigma} \sum_{i=1}^{2} V_{k i} \hat{c}_{k \sigma}^{\dagger} \hat{d}_{i \sigma}+\text { H.c. } \\
\hat{H}_{\mathcal{M}}=\sum_{i \sigma} \varepsilon_{i} \hat{d}_{i \sigma}^{\dagger} \hat{d}_{i \sigma}-t \sum_{\sigma}\left(\hat{d}_{1 \sigma}^{\dagger} \hat{d}_{2 \sigma}+\text { H.c. }\right)+\hat{H}_{2}, \\
\hat{H}_{2}=\frac{1}{2} \sum_{\left\{i_{j} \sigma_{j}\right\}} U_{i_{1} \sigma_{1} i_{2} \sigma_{2} i_{3} \sigma_{3} i_{4} \sigma_{4}} \hat{d}_{i_{1} \sigma_{1}}^{\dagger} \hat{d}_{i_{2} \sigma_{2}}^{\dagger} \hat{d}_{i_{3} \sigma_{3}} \hat{d}_{i_{4} \sigma_{4}} .
\end{gathered}
$$

The molecular ("double-dot") Hamiltonian $\hat{H}_{\mathcal{M}}$ now contains bilinear terms: the on-site energies in $\varepsilon_{i}$ and a hybridization term in $t$. The two-particle interaction term $\hat{H}_{2}$ is written here in the most general form. It can represent the Coulomb repulsion or an effectively induced interaction (e.g., kinetic exchange).

The kind of physical phenomena appearing in this model depend on the average charge state of the molecule. We focus on two situations with nearly one or two electrons. They correspond to filling fraction $\nu=1 / 4$ and $\nu=1 / 2$, where $\nu:=(1 / 4)\left\langle\sum_{i \sigma} \hat{d}_{i \sigma}^{\dagger} \hat{d}_{i \sigma}\right\rangle$. A comprehensive overview of the TIAM at arbitrary filling can be found in Logan, Wright, and Galpin (2009).

Quarter filling.-At quarter filling, the correlated subspace hosts a single electron that can take four different states. A second electron is not allowed to enter due to repulsive interactions (Coulomb blockade). A particularly interesting situation arises in the degenerate case, where all four states are energetically identical. Thus motivated we consider

$$
t=0, \quad \varepsilon_{1}=\varepsilon_{2}, \quad \text { and } \quad \Sigma_{i i^{\prime}}(\omega)=\delta_{i i^{\prime}} \Sigma(\omega) .
$$

The last expression implies that the particle leaving the molecule for excursions in the leads later returns in the same orbital. The condition ensures that the lead coupling does not lift the fourfold degeneracy. ${ }^{14}$ When charge fluctuations are suppressed due to the Coulomb blockade, the low-energy physics is described by the Kondo Hamiltonian, however, now with an SU(4) hyperspin accounting for the fourfold

\footnotetext{
${ }^{14}$ The charge exchange between molecule and reservoirs can be represented by the single-particle (hybridization) self-energy
}

$$
\Sigma_{i i^{\prime}}(\omega)=\sum_{k} V_{k i}^{*} \frac{1}{\omega-\varepsilon_{k}+\mathfrak{i} 0+} V_{k i^{\prime}}
$$

degeneracy. The associated SU(4) Kondo temperature $T_{K}^{(4)} \propto$ $e^{-1 / 2 J \rho}$ carries an extra factor of $1 / 2$ and therefore is exponentially enhanced compared to an SU(2) Kondo scale $T_{K}^{(2)} \propto e^{-1 / J \rho}$ (Bickers, 1987). Here $J=2 V^{2} /|\varepsilon|+2 V^{2} /$ $|\varepsilon+U|$, and $V^{2}$ denotes $\left|V_{k i}\right|^{2}$ averaged over the Fermi surface.

More realistic descriptions will in general contain terms violating the symmetries (37). There are two kinds of such terms. The first kind is exemplified by orbital splitting $\left(\varepsilon_{2}-\varepsilon_{1}\right)$ and magnetic field. The SU(4) physics is stable as long as these splittings remain much smaller than $k_{B} T_{K}^{(4)}$ (Borda et al., 2003; Le Hur and Simon, 2003). ${ }^{15}$ The second kind of operators are mixing terms $\left(-t+\Sigma_{12}(\omega)\right)$; these terms are relevant: when cooling below a temperature $T_{K}^{(2)}$ exponentially lower than $T_{K}^{(4)}$, there is a crossover from the SU(4) to the SU(2) fixed point ( $\operatorname{Lim}$ et al., 2006). We mention that often the $T_{K}^{(2)}$ temperature is experimentally inaccessible, and the actual observations are still determined by the SU(4) fixed point.

When the perturbations of both kinds become large enough to overcome the $k_{B} T_{K}^{(4)}$ scale, the Kondo resonance splits. The resulting peaks represent transitions within the low-energy quartet and are a residual signature of the SU(4) symmetry (Choi, López, and Aguado, 2005).

Half filling.-The TIAM at $\nu=1 / 2$ has been thoroughly investigated because it offers insights into a competition between Kondo screening and magnetic ordering. We give a brief overview of certain regimes of particular relevance for molecules. For a detailed account of the general situation, we direct the interested reader to the original research articles and reviews by Varma, Nussinov, and Van Saarloos (2002), Vojta (2006), Bulla, Costi, and Pruschke (2008), and Florens et al. (2011).

It is convenient to rewrite Eq. (36) in the following form: $\hat{H}_{2}=\sum_{i} U_{i} \hat{n}_{i \uparrow} \hat{n}_{i \downarrow}+I_{d} \hat{\mathbf{S}}_{1} \cdot \hat{\mathbf{S}}_{2}+\hat{H}_{2}^{\prime} . U_{i}$ is the energy of the on-site repulsion, $I_{d}$ is the exchange energy, and the remaining interaction terms are lumped into $\hat{H}_{2}^{\prime}$.

We consider a regime in which charge fluctuations are suppressed on each orbital. This regime is delineated by the conditions (24), with $\epsilon_{0}, U, \Gamma$ replaced by $\varepsilon_{i}, U_{i}, \Gamma_{i}$, where $\Gamma_{i}=2 \pi \sum_{k}\left|V_{k i}\right|^{2} \delta\left(\varepsilon_{k}\right)$ and $i=1,2$. Additionally, to suppress interorbital charge fluctuations, we require that $|t|,\left|\Sigma_{12}\left(E_{F}\right)\right| \ll U$ and neglect $\hat{H}_{2}^{\prime}$ for simplicity.

In this limit, it can be shown that the physics at low energies is governed by the two-impurity Kondo model (TIKM) (Jayaprakash, Krishna-murthy, and Wilkins, 1982; Žitko and Bonča, 2006). The TIKM has found applications in diverse fields, describing interactions between adatoms or double quantum dots, to mention only a few. The only remaining degrees of freedom of the molecule are the spins $\hat{\mathbf{S}}_{i}$. An effective Hamiltonian for the molecule has the form $I \hat{\mathbf{S}}_{1} \cdot \hat{\mathbf{S}}_{2}$. Here $I$ may contain contributions from direct exchange $I_{d}$, Heisenberg exchange $4 t^{2} / U$, or terms generated

\footnotetext{
${ }^{15}$ These terms couple as marginal operators in the renormalizationgroup sense.
} 
by the environment, such as Ruderman-Kittel-Kasuya-Yosida (RKKY) exchange (Jayaprakash, Krishna-murthy, and Wilkins, 1981; Proetto and López, 1981) or superexchange (Lee et al., 2010).

Upon connecting the leads, spin fluctuations are induced, as in the single-impurity Kondo effect. The resulting Kondo screening of the individual impurity, scale $T_{K}$, competes with the mutual interaction of the two spins, represented by the energy scale $I$, that is trying to pair them up.

Phenomenology of the TIKM.-Based on the previous discussion, three regimes can be distinguished (Varma, Nussinov, and Van Saarloos, 2002):

(i) At strong antiferromagnetic coupling $I \gg k_{B} T_{K}$ both spins are locked into a singlet state. The two impurities then act merely as a potential scatterer.

(ii) In the opposite limit of a strong ferromagnetic coupling $I \ll-k_{B} T_{K}$, the two spins behave as a compound spin- 1 object. If only a single screening channel applies (we specify this condition for molecular junctions in the next paragraph), the Kondo physics is of the underscreened type. Full Kondo screening is achieved close to $T=0$ if a second channel is present.

(iii) For intermediate coupling $|I| \approx k_{B} T_{K}$, we have a transition regime. The nature of the transition depends on the number of applicable screening channels (Vojta, 2006; Logan, Wright, and Galpin, 2009).

The number of screening channels (i.e., the number of available Fermi surfaces) is an important parameter that discriminates between different classes of low-energy behaviors (Nozières and Blandin, 1980). In a generic molecular junction, the molecule usually couples to a three-dimensional electrode rather than to single-channel wire. Therefore, one might suspect that in molecular junctions a description in terms of a two-channel model is more generic. While this is true in principle, this does not imply that the underscreened Kondo effect is irrelevant in molecular junctions. Namely, the two channels will not be fully equivalent. Even small differences in atomistic energy scales in general lead to significant differences in the Kondo temperatures associated with each individual channel. The reason is the exponential sensitivity of $T_{K}$ to atomistic energy scales. Therefore, with lowering temperature a wide preasymptotic regime exists exhibiting underscreened Kondo correlations even though the molecule is not coupled to a single-channel wire (Jayaprakash, Krishna-murthy, and Wilkins, 1981; Posazhennikova and Coleman, 2005). ${ }^{16}$

If only a single conduction channel needs to be taken into account, a quantum phase transition (QPT) appears in the intermediate regime (iii). The transition separates an underscreened Kondo state (doublet ground state) from the singlet regime (i) (Vojta, Bulla, and Hofstetter, 2002). A particular manifestation of the QPT in the transport is

\footnotetext{
${ }^{16} \mathrm{~A}$ hallmark of the underscreened Kondo state is the spin degeneracy of the ground state. In electron transport setups, the degeneracy is manifested by the strong splitting of the Kondo peak in a weak magnetic field (Roch et al., 2009).
}

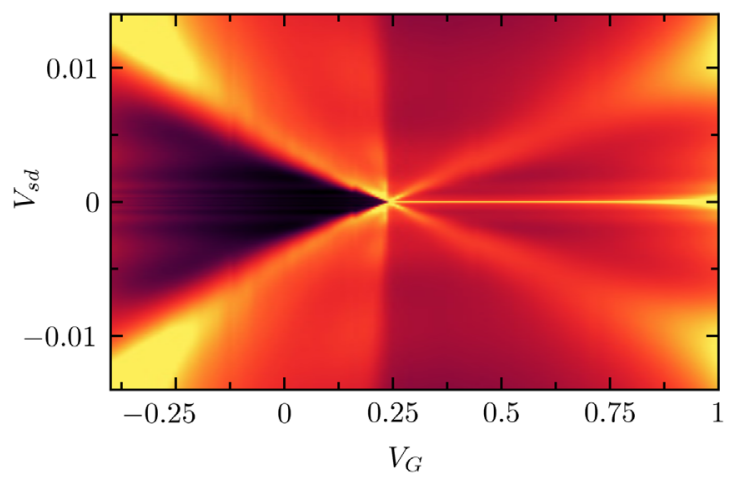

FIG. 9. The differential conductance of a TIAM near the singlettriplet transition displayed in the plane of gate or bias voltage (units, $U_{1}=U_{2}$ ). Effectively, the gate voltage fine-tunes the difference between singlet and triplet energies with $V_{G}^{*}=0.25$ at criticality. At $V_{G}>V_{G}^{*}$, a Kondo peak and triplet-singlet side peaks emerge, while $V_{G}<V_{G}^{*}$ there is a singlet gap. Color coding is such that bright yellow implies unitary conductance. From Florens et al., 2011.

the appearance of a dip in the density of states on the singlet side due to two-stage Kondo screening (Hofstetter and Schoeller, 2001). The width of the dip is a dynamically generated energy scale $T^{*} \propto \exp \left(-k_{B} T_{K} /\left|I-I_{c}\right|\right)$, where $I_{c}$ is the value of $I$ at which the QPT occurs.

Inelastic transport signatures at half filling.-The nonequilibrium dynamics of the TIAM at half filling in the Kondo regime was intensively investigated (Roura-Bas and Aligia, 2010; Florens et al., 2011). The differential conductance on the triplet side [regime (ii)] shows a Kondo peak with side peaks located at energies $\pm I$ (triplet-singlet transitions). In regime (i), there are inelastic steps corresponding to singlettriplet excitations (Paaske et al., 2006; Korytár, Lorente, and Gauyacq, 2012). As $I$ approaches $I_{c}$, the latter merge into the $\operatorname{dip}\left(k_{B} T^{*}\right)$ due to the two-stage screening. An example of a theoretical differential conductance in these regimes is shown in Fig. 9.

\section{B. Quantum interference effects}

QI features tend to be strong and robust in molecular junctions. This is hardly surprising because with a view on Eq. (19) we see that usually only a few complex valued numbers need to be added for evaluating observables. As a consequence, interfering probability amplitudes do not tend to cancel and interference effects are ubiquitous and significant. Therefore, they have received considerable attention, and proposals for applications have been made from early on (Baer and Neuhauser, 2002; van Dijk et al., 2006; Maggio, Solomon, and Troisi, 2014; Bergfield et al., 2015; Strange et al., 2015). Correspondingly, the literature on $\mathrm{QI}$ is sizable, and we focus our survey here on theoretical concepts and mechanisms. For a discussion of important experimental tests see Sec. V.D, for a basic pedagogical introduction see Hansen et al. (2009) and Lambert (2015), for an overview taking a chemical perspective see Su et al. (2016), and for a recent comprehensive review see Tsuji et al. (2018). 


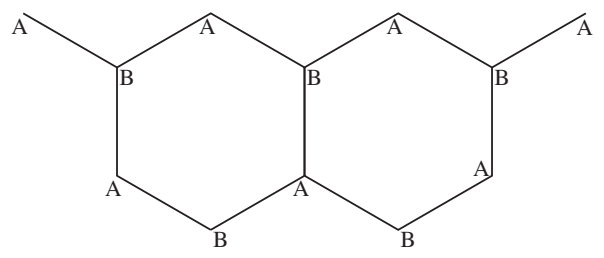

FIG. 10. Schematic illustration of a molecule with two-point contacts and the sublattice structure of the honeycomb lattice.

\section{Symmetry considerations in orbital representation}

Under certain conditions, the amplitudes appearing in the two-level model (19) cancel at some energy $E$, so the transmission vanishes and is strongly suppressed nearby; a Fano (anti)resonance appears. It is this manifestation of destructive interference that is mostly discussed in the context of molecular transport. An intuition for the effect can be obtained by evaluating Eq. (2) for a simple model system. We consider a tight-binding representation of a molecule with the source contacting a single site and the drain contacting another single site only; see, e.g., Fig. 10. Then the matrices $\Gamma_{\mathcal{L}}, \Gamma_{\mathcal{R}}$ reduce to numbers and

$$
\mathcal{T}(E)=\Gamma_{\mathcal{L}} \Gamma_{\mathcal{R}}|\langle d|G(E)| s\rangle|^{2} \equiv \Gamma_{\mathcal{L}} \Gamma_{\mathcal{R}}\left|G_{d s}(E)\right|^{2},
$$

where $s, d$ denote the contact sites for the source (left) and drain (right). The Green's function $G_{d s}(E)$ describes the probability amplitude for a particle with energy $E$ (measured with respect to $\epsilon_{F}$ ) to travel from source to drain, while the prefactor $\Gamma_{\mathcal{L}} \Gamma_{\mathcal{R}}$ defines the contact resistance. When comparing to experiments, the latter can be conveniently dealt with by investigating suitable conductance ratios in which the contact conductance cancels (Geng et al., 2015; Manrique et al., 2015).

We evaluate Eq. (38) by using explicit representations of the matrix element $G_{d s}(E)$. In the limit of weak coupling, we can neglect excursions into the leads so that $G_{d s}$ has a simple representation in terms of the states of the isolated molecule:

$$
G_{d s}^{(0)}(E)=\sum_{n} \frac{\psi_{d, n}^{*} \psi_{s, n}}{E-\epsilon_{n}+\mathfrak{i} 0} .
$$

Here $\psi_{s, n}, \psi_{d, n}$ denote the amplitudes of orbitals with energy $\epsilon_{n}$ at the molecular sites that make contact with source and drain. In the spirit of the two-level model, we now assume that only two orbitals are relevant, HOMO and LUMO:

$$
G_{d s}^{(0)}(E) \approx \frac{\psi_{d, H}^{*} \psi_{s, H}}{E-\epsilon_{H}+\mathfrak{i} 0}-\frac{\psi_{d, L}^{*} \psi_{s, L}}{\epsilon_{L}-E-\mathfrak{i} 0}
$$

Let us, for simplicity, assume the wave functions to be real valued in the preceding expression. One then realizes that a tendency for cancellation exists if $\epsilon_{H}<E<\epsilon_{L}$, provided that the products in the numerator have the same sign. The interesting aspect of this trivial observation is that for generic and relevant classes of organic molecules this relative sign can be easily predicted (Tada and Yoshizawa, 2002, 2015; Yoshizawa, Tada, and Staykov, 2008; Lovey and Romero, 2012).

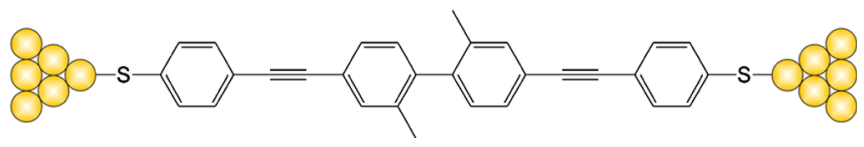

FIG. 11. Double-dot molecule used in the experiment (Ballmann et al., 2012). Owing to the presence of the side groups, the center benzene rings are tilted against each other, leading to a partial decoupling of the left and right $\pi$ systems.

Mirror plane, inversion symmetry.-Molecular junctions (molecule plus the source-drain contacts) can exhibit discrete spatial symmetries, e.g., mirror planes and inversion centers. Even though under typical experimental conditions such symmetries are only approximate, they still can have an important impact on the transmission. Specifically, we consider a parity symmetry that allows us to sort wave functions into even and odd behavior under reversal of left and right. For broad molecular classes, in particular, for molecular wires, the generic situation is that two wave functions neighboring in energy exhibit different parity. ${ }^{17}$ This typically implies that, e.g., the HOMO is symmetric (even) while the LUMO is antisymmetric (odd). In this situation, the numerators in Eq. (40) exhibit opposite signs. QI is constructive if the denominators also exhibit the same signs, which is the case if $\epsilon_{H}<\epsilon_{F}<\epsilon_{L}$.

Molecular double dots.-A special class of molecular junctions comprises molecules that consist of two identical, weakly coupled subunits (double dots). In such systems, molecular orbitals tend to form parity doublets with respect to mirroring the two dots. For weakly coupled double dots arranged in series, the parity doublets hardly split in energy and therefore can exhibit strong QI features. Owing to the smallness of the splitting, the doublet states tend to be either both occupied or both empty. Under this condition, interference typically is destructive. This case is realized in molecule 3, which was investigated by Ballmann et al. (2012) (see Fig. 11), at least if screening is strong enough to suppress charging effects. The molecule exhibits a left and a right side with $\pi$ systems that have been partially decoupled by tilting the systems against each other, reducing the bond to a $\sigma$ bond.

A situation with pronounced QI can also arise for two quantum dots arranged in parallel. If the dots are not too weakly coupled, then the occupied states near $\epsilon_{F}$ exhibit even parity such that orbital contributions from each dot add constructively. Hence, the transmission is seen to be enhanced up to a factor of 2 as compared to the parallel transport through two independent single dots. The experimental demonstration of this effect by Vázquez et al. (2012) is discussed in Sec. V.D.

Sublattice symmetry.-We specialize on the important class of molecules with only one relevant orbital per atom, including graphene-type molecular matter, e.g., flakes, nanoribbons, and nanotubes $\left(p_{z}\right.$ orbital). Such systems can exhibit a

\footnotetext{
${ }^{17}$ Since in longer wires the longitudinal direction accommodates most of the phase space, most states differ in the quantum numbers associated with this direction. "Generic" here accounts for the fact that for this reason most states neighboring in energy (but not all) differ in their longitudinal (parity-based) quantum numbers.
} 
bipartite symmetry such that each atom ("site") can be associated with one of two sublattices, $A$ or $B$ (see Fig. 10), corresponding to starred and nonstarred sites in chemistry nomenclature (Tsuji et al., 2014, 2018). The associated tight-binding Hamiltonian (also called the connectivity matrix in graph theory) can be cast into the form

$$
H_{\mathrm{ch}}=\left(\begin{array}{cc}
0 & t \\
t^{\dagger} & 0
\end{array}\right)
$$

The matrices $t, t^{\dagger}$ are $N_{A} \times N_{B}$ and $N_{B} \times N_{A}$, respectively. $t$ describes a hopping process $B \rightarrow A$ and $t^{\dagger}$ accounts for the time-reverse process. The on-site energies are taken to be all the same and zero, assuming that all sites are equivalent; they act as the reference letting $\epsilon_{F}=0$ at half filling. As a consequence of the sublattice symmetry (also known as "chiral" symmetry), eigenvalues of $H_{\mathrm{ch}}$ come in pairs $\pm \epsilon$ ("Coulson-Rushbrooke pairs"). Moreover, if $\chi=(a, b)$ denotes an eigenstate of $H_{\mathrm{ch}}$ with energy $\varepsilon$ and amplitudes $a, b$ on sublattices $A, B$, then $\chi=(a,-b)$ is the corresponding eigenstate for $-\epsilon$.

With respect to QI and Eq. (40), the sublattice symmetry has the following trivial implications: (i) $\epsilon_{H}=-\epsilon_{L}$ at half filling (i.e., with one electron per site). (ii) The relative signs of the two numerators in Eq. (40) depend on whether or not the source and drain are located on the same sublattice: if the source and drain sites belong to the same sublattice, the relative sign between the HOMO and LUMO states is the same and the interference is destructive. In contrast, when two different sublattices are contacted the interference is constructive. For the transmission function, this argument was first made by Solomon, Andrews, Goldsmith et al. (2008). The sensitivity of the transmission to the relative choice of the contact lattice was discussed by Tsuji et al. (2014) and Zhao, Geskin, and Stadler (2017). Powerful selection rules have been worked out that we rederive and discuss later.

In more complete descriptions of organic molecules, such as $a b$ initio calculations, the sublattice symmetry (41) tends to be approximate, due to the presence of next-nearest-neighbor terms and inhomogeneous on-site potentials and also due to additional bands, e.g., $\sigma$ bands. The latter give rise to superimposing transport channels that effectively mask the Fano dip (Ke, Yang, and Baranger, 2008). The leading effect of higherorder hopping terms is to disturb the energy pairing, while the nodal structure of the $\pi$-electron orbitals is less affected. As a consequence, with higher-order hopping terms the Fano dip is no longer situated symmetrically between the HOMO and the LUMO resonance.

Without going into further detail, we mention an interesting development related to cases where a splitting of the Fano resonance was reported (Solomon et al., 2011). A detailed investigation of this effect using a linear tight-binding chain as a paradigm was performed by Tsuji et al. (2014). It was found that two kinds of Fano resonances occur that differ, e.g., in the way in which they react to perturbations; see Sec. IV.B.3. While the first kind is easily understandable within a picture of molecular orbitals, the second kind requires a more elaborate analysis based on graphical methods, see Sec. IV.B.2 (Zhao, Geskin, and Stadler, 2017).

\section{Sum-over-paths approach}

In Eq. (39) the Green's function $G_{d s}^{(0)}$ is represented as a sum over its poles ("Lehmann representation"). There is an alternative representation of $G_{d s}^{(0)}$ in terms of determinants,

$$
G_{d s}^{(0)}(E)=\frac{\operatorname{det}\left(E-H_{\mathcal{M}}\right)_{d, s}}{\operatorname{det}\left(E-H_{\mathcal{M}}\right)},
$$

where $H_{\mathcal{M}}$ denotes the tight-binding Hamiltonian of the isolated molecule. The truncated matrix $\left(E-H_{\mathcal{M}}\right)_{r, c}$, i.e., the "minor," derives from the parent matrix $\left(E-H_{\mathcal{M}}\right)$ by eliminating the row $r$ and the column $c$. Writing Eq. (42) we anticipate that $\operatorname{det}\left(E-H_{\mathcal{M}}\right) \neq 0$, so there is no spectral weight of $H_{\mathcal{M}}$ at the energy $E$. Equation (42) and variants thereof have been used as a starting point to derive graphical rules for predicting the presence of Fano features (Pickup and Fowler, 2008; Fowler et al., 2009; Markussen, Stadler, and Thygesen, 2010, 2011; Mayou, Zhou, and Ernzerhof, 2013; Stuyver, Fias et al., 2017).

The Markussen-Stadler-Thygesen (MST) rules.-A general set of rules to exploit Eq. (42) for deriving the transmission at zero energy $E=0$ was obtained by Markussen, Stadler, and Thygesen (2010). We rederive their result.

A determinant of an arbitrary $N \times N$ matrix $H$ with elements $h_{i j}(i, j=1, \ldots, N)$ has an explicit representation

$$
\operatorname{det} H=\sum_{\sigma} \operatorname{sgn}(\sigma) \prod_{i=1}^{N} h_{i \sigma(i)},
$$

with $\sigma$ abbreviating a permutation of the numbers $1, \ldots, N$ and $\operatorname{sgn}(\sigma)=1$ for even permutations and $\operatorname{sgn}(\sigma)=-1$ for odd ones. We view $H$ as the connectivity matrix of a graph with sites $i$ and $j$ and with connectivities $h_{i j}$ indicating a directed link from $j$ to $i$. In this picture, individual terms appearing in Eq. (43) can be interpreted as paths on the graph. For instance, in the case of $N=3$ sites, the term $h_{12} h_{23} h_{31}$ appears. It has the interpretation of a loop that starts from the first site, then visits the third, visits the second next, and eventually returns to the starting point. To stay within the picture of loops, we interpret a diagonal element $h_{i i}$ as an undirected loop from a site into itself.

Then the entire determinant (43) has a transparent graphical representation: it is a summation over all directed paths on the graph that have the property that there is one incoming and one outgoing link per site. It is thus clear that each path consists of one or more loops (Harary, 1962).

Following Markussen, Stadler, and Thygesen, we translate the graphical rules for determinants into a statement about the minor $\operatorname{det}\left(H_{\mathcal{M}}\right)_{d, s^{*}}$. To this end, we use the familiar fact that determinants can be calculated by expanding into such minors. Suppose that the connectivity matrix $H_{\mathcal{M}}$ features a direct link between source and drain $h_{d, s}$. Then performing an expansion in terms of minors, the determinant $\operatorname{det} H_{\mathcal{M}}$ needs to contain a term $h_{d, s} \operatorname{det}\left(H_{\mathcal{M}}\right)_{d, s}$. Recalling 
that only closed paths contribute, this term can be nonvanishing only if its associated graphical representation contains at least one path with only closed loops. Correspondingly, we conclude that the minor can be nonvanishing only, $\operatorname{det}\left(H_{\mathcal{M}}\right)_{d, s} \neq 0$, if the following two conditions are met by its representation in terms of paths on the graph: (i) there is a path connecting source and drain (which is closed eventually by $h_{d, s}$ ). (ii) All sites that do not belong to the path that connects source and drain are bound in one or more closed loops.

The graphical rules of Markussen, Stadler, and Thygesen incorporate these two conditions: consider the atom positions of the molecule as sites of a graph. Check to see if a path can be found on that graph that satisfies (i) and (ii); if there is no such path, then $\operatorname{det}\left(H_{\mathcal{M}}\right)_{d, s}=0$ and there is no transmission at zero energy (Markussen, Stadler, and Thygesen, 2010).

The original derivation was given for the case where $H_{\mathcal{M}}$ features zero on-site energies only. In this case, paths with isolated sites have zero weight and hence are not considered closed. We emphasize that the rules derived here are completely general and do not, in particular, require the connectivity matrix $H_{\mathcal{M}}$ to be bipartite (Xia et al., 2014; Stadler, 2015; Strange et al., 2015).

Zero eigenvalues and radicals.-We extend the discussion of the sum-over-paths approach preparing relations that become important in Sec. IV.B.3. The representation of determinants as sums over terms that represent closed loops can have interesting implications for the evaluation of $\operatorname{det}\left(E-H_{\mathcal{M}}\right)$. Namely, it may in fact not be possible to find a closed path: even with the "best" dressing of the graph with directed links, there may always be certain sites left that cannot be made to participate in any loop. In that case, we safely conclude that $\operatorname{det}\left(H_{\mathcal{M}}\right)=0$, so there are zero eigenvalues.

The smallest number of such isolated sites ("radicals") that one can achieve we call $\zeta_{r}$. This number constitutes a lower bound for the multiplicity of the zero-energy root, and hence also for the number of zero-energy eigenstates: $\zeta \geq \zeta_{r}$.

Bipartite symmetry: Double bonds.-In the presence of bipartiteness, e.g., for hydrocarbons with alternating single-double bonds, a lower bound for $\zeta_{r}$ can be derived. Suppose that the $\operatorname{det} H_{\mathcal{M}}$ is nonvanishing. Then there must be at least one closed path, potentially featuring disconnected loops, that touches every graph site once and only once. Because of bipartiteness, each loop of this path visits every sublattice in an alternating fashion, and therefore always contains an even number of sites. We conclude that $\operatorname{det} H_{\mathcal{M}}$ can be nonvanishing only if the following necessary condition is met: the number of sites (atoms) is the same in each sublattice $N_{A}=N_{B}$. Based on the analysis of the preceding paragraph, a lower bound $\zeta_{r} \geq\left|N_{A}-N_{B}\right|$ is thus derived.

We can give a practical guide to a better estimate, however. Consider one of the paths that features the minimum number of radicals; it exhibits one or more loops and a number of $\zeta_{r}$ isolated sites. Every one of the loops can be decorated by double bonds following the rule that along the loop every site should participate in one and only one double bond. In this way, paths on bipartite lattices are associated with a decoration of a number of $N_{\mathrm{db}}$ double bonds. We derive for the number of radicals $^{18}$

$$
\zeta_{r}=N_{A}+N_{B}-2 N_{\mathrm{db}}
$$

\section{Selection rules for destructive $Q I$}

The MST rules are somewhat tedious to handle for larger molecules because of bookkeeping for a combination of two geometrical objects: closed loops and an open path. This is a remnant of the fact that the minor equation (42) is evaluated directly. Working with minors can be avoided by exploiting determinant relations. They allow us to express the ratio (42) in terms of determinants of proper connectivity matrices. Thus, a sum-over-path analysis of the transmission involves only sums over closed paths.

We define $\Delta_{r, c}^{(0)}(E)=\operatorname{det}\left(E-H_{\mathcal{M}}\right)_{r, c}$ such that $G_{d s}^{(0)}(E)=$ $\Delta_{d, s}^{(0)}(E) / \Delta^{(0)}(E)$. Using a general identity for determinants (Fowler et al., 2009), we can relate the determinant of the minor $\Delta_{d, s}^{(0)}$ to three new determinants in the following manner:

$$
\Delta_{d s, d s}^{(0)}=\left[\Delta_{d, d}^{(0)} \Delta_{s, s}^{(0)}-\left(\Delta_{d, s}^{(0)}\right)^{2}\right] / \Delta^{(0)}
$$

This expression is attractive because the new determinants have an appealing graphical interpretation: $\Delta_{d, d}^{(0)}, \Delta_{s, s}^{(0)}$ represent determinants of the matrix $\left(E-H_{\mathcal{M}}\right)$ with one site, $d$ or $s$, removed; similarly, $\Delta_{d s, d s}$ is a determinant with source and drain sites removed from $\left(E-H_{\mathcal{M}}\right)$. Evaluating with the help of Eq. (38) then allows us to write

$$
\mathcal{T}(E)=\Gamma_{\mathcal{L}} \Gamma_{\mathcal{R}}\left[\Delta_{d, d}^{(0)} \Delta_{s, s}^{(0)}-\Delta^{(0)} \Delta_{d s, d s}^{(0)}\right] / \Delta^{(0) 2}+\cdots,
$$

valid to lowest order in $\Gamma_{\mathcal{L}}, \Gamma_{\mathcal{R}}$. Such a relation was derived previously by Stuyver et al. (2015), embarking on earlier work by Pickup and Fowler (2008) and Fowler, Pickup, and Todorova (2011).

Bipartite symmetry.-We apply Eq. (45) to molecules with bipartite symmetry, such as alternating hydrocarbons, and focus on the band center $E=0$. To meet the condition $\Delta^{(0)}(0) \neq 0$, we require that the number of sites in each sublattice is the same: $N_{A}=N_{B}$. In other words, the $N_{A} \times N_{B}$ matrix $t$ must be square. As we saw earlier, otherwise $H_{\mathcal{M}}$ exhibits at least $\left|N_{A}-N_{B}\right|$ zero-energy states. This statement was first derived by Longuet-Higgins (1950). By the same argument, we conclude that $\Delta_{d, d}^{(0)}, \Delta_{s, s}^{(0)}=0$ at $E=0$ : the removal of the drain or the source site implies a sublattice imbalance with $N_{A} \neq N_{B}$, and hence the existence of at least one zero eigenvalue.

\footnotetext{
${ }^{18}$ We mention that the number of radicals $\zeta_{r}$ is closely related to the number of Kekule structures $K$ that are associated with a graph. In particular, if $K>0$, then $\zeta_{r}=0$.
} 
We thus arrive at the relation

$$
\mathcal{T}(0)=-\Gamma_{\mathcal{L}} \Gamma_{\mathcal{R}} \frac{\Delta_{d s, d s}^{(0)}(0)}{\Delta^{(0)}(0)}
$$

Its consequences have been investigated (Fowler et al., 2009; Stuyver, Fias et al., 2017). Toward deriving rules for QIinduced transmission zeros, one arrives at the fact that Eq. (46) expresses the transmission as a ratio of determinants corresponding to matrices that can both be interpreted as Hamiltonians of a physical system. In particular, $\Delta_{d s, d s}$ corresponds to the original system with two vertices deleted (vacancies in the nomenclature of material sciences) at the original position of source and drain.

For nearest-neighbor hopping, one can relate $\mathcal{T}(0)$ to known spectral properties employing graphical rules that date back to the early work of Longuet-Higgins (1950); we have rederived them using the sum-over-path approach: the number of zero modes $\zeta$ associated with $H_{\mathcal{M}}$ and $\left(H_{\mathcal{M}}\right)_{d s, d s}$ is given by

$$
\zeta \geq N_{A}+N_{B}-2 N_{\mathrm{db}}
$$

where $N_{\mathrm{db}}$ denotes the maximum number of double bonds that can be placed on the graph.

One discriminates between two kinds of zero modes. The predictable modes result from a sublattice imbalance: $\zeta^{\text {pre }}=\left|N_{A}-N_{B}\right|$. The remaining modes, called supernumerary, come in pairs

$$
\zeta^{\sup } \geq 2\left[\min \left(N_{A}, N_{B}\right)-N_{\mathrm{db}}\right]
$$

and $\zeta=\zeta^{\text {pre }}+\zeta^{\text {sup }}$. The result was sharpened later in benzoidal graph theory: the equal sign holds for honeycomb lattices (Fajtlowicz, John, and Sachs, 2005). We emphasize that supernumerary modes are far from a mere curiosity. For instance, as has been demonstrated, they play an important role for the thermodynamic properties of graphene flakes (Häfner et al., 2014). Such supernumerary modes are associated, e.g., with incomplete parts of the honeycomb lattice at the edges, as we illustrate later.

Thus prepared, we distinguish in the discussion of Eq. (46) among three cases:

(i) The case $\Delta^{(0)}(0) \neq 0$ with source and drain located in the same sublattice.-Since the parent Hamiltonian $H_{\mathcal{M}}$ exhibits balanced sublattices, the truncated Hamiltonian $\left(H_{\mathcal{M}}\right)_{d s, d s}$ is imbalanced, $\zeta^{\text {pre }}=2$. There are at least two zero modes, so $\Delta_{d s, d s}^{(0)}(0)=0$ and $\mathcal{T}(0)$ vanishes. We thus confirm the qualitative findings based on wave function arguments and make them rigorous within the Hückel model.

(ii) The case $\Delta^{(0)}(0) \neq 0$ with source and drain located in different sublattices. - The truncated Hamiltonian $\left(H_{\mathcal{M}}\right)_{d s, d s}$ is balanced; the presence of supernumerary zero modes can be checked by graphically employing Eq. (48) for the case of benzenoids. Certain substructures (motifs) of alternating hydrocarbons, like dangling bonds, can contribute such a

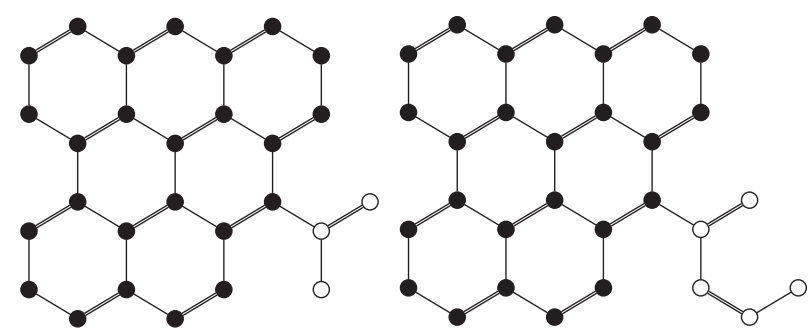

FIG. 12. Two examples of edge structures that are always associated with supernumerary zero modes. (Left panel) The two singly connecting sites share the same binding partner. Therefore, only one of them can form a double bond; the other becomes a radical. (Right panel) A similar pattern. From Weik et al., 2016.

mode. By "dangling bond" we refer to a situation where a lattice site couples to a single other site; a realization is found in cross-conjugated molecules (Solomon, Andrews, Goldsmith et al., 2008). Further examples of such motifs were listed by Weik et al. (2016). However, cross conjugation by itself is not a reliable indicator of destructive interference (Pedersen et al., 2015). For instance, if dangling bonds come in pairs, the respective zero modes can hybridize and split away from zero energy. In this case, a Fano dip survives only to the extent that hybridization can be considered extremely weak. We mention in this context that special classes of edge motifs can be identified that are always accompanied with a zero mode (Weik et al., 2016). Examples from Weik et al. (2016) are reproduced in Fig. 12.

(iii) The case $\Delta^{(0)}(0)=0$.-This situation has received much less attention thus far. A first discussion of the situation was given by Stuyver, Fias et al. (2017). We emphasize that a vanishing denominator in Eq. (42) at $E=0$ is far from pathological: (i) a generic parent Hamiltonian $H_{\mathcal{M}}$ also exhibits supernumerary zero modes if it is balanced, and (ii) Eq. (42) is written for the isolated molecule because only a gapped spectrum can satisfy, in principle, the condition of zero spectral weight at a given energy $E$.

We recall the exact relation

$$
\mathcal{T}(E)=-\Gamma_{\mathcal{L}} \Gamma_{\mathcal{R}}\left|\Delta_{d, s}(E) / \Delta(E)\right|^{2},
$$

where $\Delta=\operatorname{det}\left(E-\tilde{H}_{\mathcal{M}}\right)$ and $\Delta_{d, s}=\operatorname{det}\left(E-\tilde{H}_{\mathcal{M}}\right)_{d, s}$ with $\tilde{H}_{\mathcal{M}}=H_{\mathcal{M}}+\Sigma_{\mathcal{M}} ; \Sigma_{\mathcal{M}}$ denotes the self-energy accounting for both electrodes. Since the molecule exhibits only a single contact orbital for the source and drain, its transmission must be bounded, $\mathcal{T}(E) \lesssim 1$, for any energy and electrode coupling. This implies that wherever the polynomial in the denominator exhibits a root, there must be a corresponding root with the same or higher multiplicity also in the numerator in the limit of vanishing coupling, i.e., in Eq. (45).

We thus conclude that even in the case where $H_{\mathcal{M}}$ exhibits zero eigenvalues, the main ideas of the analyses outlined here remain valid. Basically, the multiplicity of the roots of two 
<smiles>O=C1c2ccccc2C(=O)c2ccccc21</smiles>

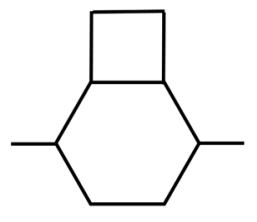

FIG. 13. Structures of (left panel) the molecules anthraquinone and (right panel) the molecule LC2. From Pedersen et al., 2015.

polynomials has to be determined with graphical rules. For bipartite systems, this implies counting radicals. If the number of radicals in the numerator determinants of Eq. (45) exceeds the one in the denominator, then the transmission at zero energy is suppressed.

The precise value of the transmission depends on the cutoff mechanism. It can be determined by small violations of the symmetric form (41) as they are brought about by nextnearest-neighbor terms or variations in on-site potentials. Alternatively, the tunnel coupling $\Gamma=\mathfrak{i}\left(\Sigma_{\mathcal{M}}-\Sigma_{\mathcal{M}}^{\dagger}\right)=\Gamma_{\mathcal{L}}+$ $\Gamma_{\mathcal{R}}$ can also serve as a cutoff; for the first discussion see Stuyver, Fias et al. (2017).

\section{Applications}

We illustrate the implications of the rule (46) by applying it to the three molecules shown in Figs. 13 and 14: (a) the anthraquinone molecule, (b) the LC2 molecule of Pedersen et al. (2015), which is a combination of a carbon six-site ring and a four-site ring, and (c) the azulene molecule (five-seven carbon double ring). ${ }^{19}$

(a) We consider both oxygen sites of the anthraquinone to be dangling and, following Markussen, Schiötz, and Thygesen (2010) and Guédon et al. (2012), we attach the source and drain to different sublattices. The molecule exhibits a benzoid structure with sublattice symmetry. We have $\zeta^{\text {sup }}=0$ for the parent Hamiltonian, so $\Delta^{(0)}(0) \neq 0$. However, after eliminating the source and drain the truncated Hamiltonian has $\zeta^{\text {sup }}=2$, so $\Delta_{d s, d s}^{(0)}(0)=0$. Consistent with this result, a Fano dip is seen in the model calculations. When the dangling bonds are removed, no supernumerary modes are found and Fano dips are not expected, which is in agreement with Markussen, Schiötz, and Thygesen (2010), Guédon et al. (2012), and Stuyver, Fias et al. (2017). Since supernumerary zero modes come in pairs, a small perturbation added to $H_{\mathcal{M}}$ will tend to lift this degeneracy and split the Fano resonance. This prediction is in agreement with observations; see the related discussion in Sec. IV.B.1.

\footnotetext{
${ }^{19}$ While anthraquinone was originally proposed as a candidate for a redox switch (van Dijk et al., 2006), subsequent theoretical work revealed a strong variation of the transmission with energy near $\epsilon_{F}$, which could serve as a gate-driven switch even in the absence of a genuine redox reaction (Markussen, Schiötz, and Thygesen, 2010). When switching was later confirmed experimentally, the observed effect was then attributed to electrochemically controlled QI (Darwish et al., 2012).
}
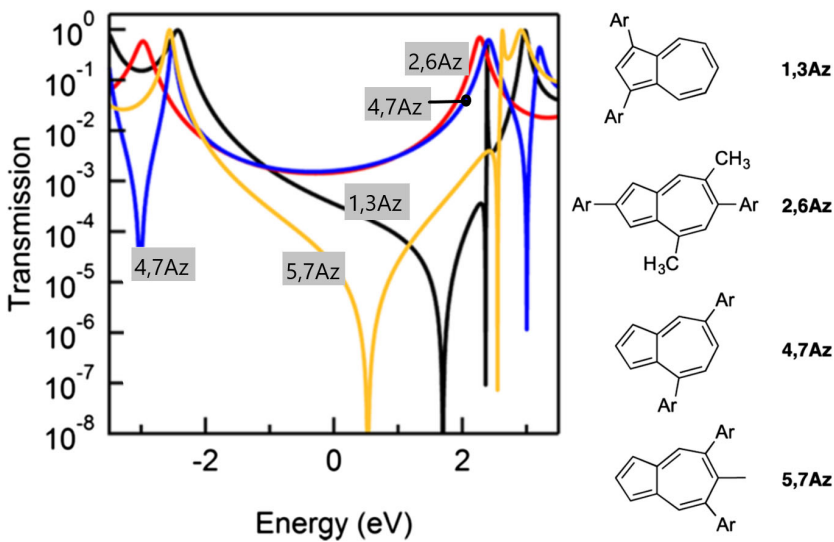

FIG. 14. Azulene with different positions of the linker group (denoted by "Ar-") and corresponding ab initio transmissions. The molecule is not bipartite, so the $a b$ initio transmissions exhibit nodes shifted from the gap center, in agreement with the rules discussed in the text. From Xia et al., 2014.

(b) LC2 carries contacts in paraposition at the six ring (Fig. 13) and also exhibits a sublattice symmetry. Since it is balanced, there are no predictable zero modes. However, the graph is not within the honeycomb class and Eq. (48) does not reduce to an equality; one needs to check for supernumerary zero modes explicitly. While the parent graph turns out to have none, the truncated graph exhibits two supernumerary modes associated with the four ring. Hence, we predict destructive interference, which is consistent with Pedersen et al. (2015).

(c) Following Xia et al. (2014) and Schwarz et al. (2016), we consider the azulene molecule (Fig. 14). This molecule is not bipartite, and therefore the full equation (45) must be used. We first notice that the molecule is conjugated, i.e., there is a consistent covering of the graph with double bonds. This implies the existence of a closed loop and therefore allows $\operatorname{det} H_{\mathcal{M}} \neq 0$. For the transmission, we consider contacts at positions 1 and 3 (Fig. 14). Then, after removing the source 1 and the drain 3 , the graph exhibits an isolated site 2 ; hence, $\Delta_{d s, d s}^{(0)}(0)=0$. Further, removing just one contact site, either 1 or 3 , the resulting graph can be covered with a loop for the seven ring and a double bond between the two sites remaining from the five ring. Hence, the determinants $\Delta_{d, d}^{(0)}, \Delta_{s, s}^{(0)}$ may be nonvanishing and there is no prediction. As it turns out, there is indeed no Fano dip at zero energy seen in this case (Xia et al., 2014; Stadler, 2015; Schwarz et al., 2016); see Fig. 14.

(d) Equation (46) also applies to hexagonal graphene nanoflakes (Valli et al., 2018, 2019). As one would expect, if the source and drain couple to the same sublattice ("meta"), a pronounced destructive QI is observed that is absent otherwise ("para" and "ortho"). Valli et al. proposed using this effect for spin and valley filtering in electronic transport. 


\section{QI and ring currents}

The scattering states that enter the Landauer-Büttiker picture are not invariant under time reversal. For this reason, they generically support circulating ("ring" or transverse) currents, unless these are suppressed by extra symmetries, such as mirror planes. They give rise to local, bias-induced magnetic fields and thus are physical observables that enjoy a unique definition (Walz, Wilhelm, and Evers, 2014). A discussion was given by Rai, Hod, and Nitzan (2010). Their experimental detection has not yet been achieved, presumably because eddies are strongest on the atomic scale, where they are hard to resolve. However, a proposal has been made on how the phenomenon could be studied experimentally in slightly larger, mesoscopic systems, where they actually might have a profound effect, for instance, on spin-relaxation times (Walz, Wilhelm, and Evers, 2014).

Circulating currents are prevalent close to a Fano resonance, where the transport (longitudinal) current $I_{\mathrm{tr}}$ is suppressed. Their qualitative behavior is discussed conveniently within a toy model (Walz, Wilhelm, and Evers, 2014). At energies $E$ near resonance $\epsilon_{c}$, we have for the ratio of circulating to transport current $I_{\text {circ }} / I_{\text {tr }} \propto\left(E-\epsilon_{c}\right)^{-1}$, which indicates that ring currents can exceed the transport currents by orders of magnitude. In graphene samples with adatoms, eddies with enhancements of 2 to 3 orders of magnitude have been computed (Walz, Wilhelm, and Evers, 2014). Note that the ring current switches sign when $E$ passes by the resonance, which is consistent with explicit model calculations (Rai, Hod, and Nitzan, 2010; Solomon et al., 2010).

Because of their robust nature and strong signatures, ring currents have motivated a significant amount of theoretical research on transport in ring-shaped molecular systems and, in particular, in Aharonov-Bohm-type geometries. This includes the effect of magnetic fluxes on occupation numbers and the current-voltage characteristics (Rai, Hod, and Nitzan, 2011, 2012; Bedkihal, Bandyopadhyay, and Segal, 2013) and the interplay of spin-flip scattering and circular currents (Rai and Galperin, 2012). Transient phenomena have been investigated in detail (Tu et al., 2012, 2016; Schoenauer et al., 2019), and effects of electronic correlations have also been addressed (Nuss, von der Linden, and Arrigoni, 2014).

\section{Temperature and interaction effects}

The QI effects discussed thus far reflect the nodal structure of molecular wave functions. Changing this structure costs energies that correspond to purely electronic excitations. Therefore, qualitatively QI tends to be robust against electron-electron interactions and thermal fluctuations in small molecules (Cardamone, Stafford, and Mazumdar, 2006; Markussen and Thygesen, 2014). A careful study of the Pariser-Parr-Pople model, which simulates interactions in $\pi$ systems, resulted in a similar conclusion (Pedersen et al., 2014).

The most obvious effect of temperature on the transmission is smearing of the Fano dip, indicating an incoherent averaging over a thermal ensemble of molecular structures. Even though conceptually straightforward, the effect is somewhat tedious to describe $a b$ initio because many different vibrations are involved.

We continue by describing additional, more subtle interference effects that appear only with interactions:

Vibrations.-A special situation can arise near degeneracies, where otherwise weak interactions can have significant effects. For instance, a coupling to vibrations can enhance the inelastic scattering rate so as to significantly weaken QI if interference is brought about by two nearly degenerate levels (Härtle et al., 2011). The temperature dependence of QI in an anthraquinone has been attributed to this mechanism (Rabache et al., 2013). Conversely, it has been reported that QI can also selectively suppress signatures of vibrational modes in inelastic electron tunneling spectroscopy (IETS) spectra (Lykkebo et al., 2014). The interplay of vibrations and destructive interference has also been investigated with graph-theoretical means (Sýkora and Novotný, 2017).

Electron-electron interactions and many-body effects.Much of the intuition that has been developed for QI in molecules is based on tight-binding models. However, manybody effects have been identified that are not captured by effective single-particle descriptions.

(a) Interaction effects lead to extra poles in the Green's function that indicate the existence many-body excitations. Such poles inevitably interfere with each other when being summed over in the construction of the many-body Green's function. An illustrative example is given by the Anderson model, where many-body excitations of a localized level emerge as lower and upper Hubbard peaks; see Eq. (27). In this case, QI manifests as an extra Fano resonance, a "Mott node" in the terminology of Bergfield et al. (2011). In the context of many-body degeneracies, which appear in models of coupled quantum dots, more complicated interference scenarios can also be realized (Donarini, Begemann, and Grifoni, 2010; Niklas et al., 2017). Of special interest is the Abrikosov-Suhl pole that is brought about by the Kondo effect. Like any other pole of the Green's function, it can give rise to interference phenomena. The most recent discovery in this context is the Kondo blockade discovered by Mitchell et al. (2017) and discussed in Sec. IV.A.2.

(b) While the notion of molecular orbitals is robust against interaction effects (Pedersen et al., 2014), the energy ordering of orbitals can be modified as a consequence of strong Coulomb interactions. This has a strong impact on the relative weight and phase of the interfering poles in the many-body Green's function and therefore crucially enters QI. Observation of this rather striking effect in STM experiments was reported by Yu et al. (2017).

\section{KEY EXPERIMENTAL RESULTS AND THEIR SEMIQUANTITATIVE UNDERSTANDING}

Before we turn to a quantitative comparison between theory and experiment in Sec. VI, it will be useful to highlight a 
number of results that illustrate the level of qualitative, or semiquantitative, understanding that we have achieved. We do not now attempt a full overview of the literature of singlemolecule transport, but we focus on results that uncover systematic trends and important physical effects in molecular junctions. By this overview we illustrate an important conclusion: despite the many unknown and poorly understood factors listed in Secs. II and III, many of the qualitative features have been understood. This implies that such features are robust against variations in electrode configurations and molecule-electrode bonding patterns and other poorly known factors, and that they are robust against the approximations made in developing the theory. It will be interesting to investigate why we find this robustness, but also to probe under which circumstances this breaks down. Roughly speaking, this section is devoted to the robustness and Secs. VI and VIIexplore the limits of validity and breakdown of this robustness.

\section{A. Conductance as a function of length}

The foremost systematic characteristics studied for molecular wires is the length dependence of the conductance. The case of alkanes has been investigated extensively and serves for us as a paradigm (Akkerman and de Boer, 2008). In addition to these carbon-based wires, wires based on other elements, such as Si (silanes) and Ge (germanes), have been studied with qualitatively similar conclusions ( $\mathrm{Su}$ et al., 2017); an overview may be found in Su et al. (2016), Table 1, and Gunasekaran et al. (2018).

In the review by Akkerman and de Boer (2008), the data obtained from many measurement techniques were compared as a function of the number $N$ of carbon atoms in the chain, ranging from $N=2$ to 28 . These data are discussed more quantitatively in Sec. VI. Of relevance to us is the observation that the conductance decreases typically exponentially with the number $N$, i.e., as a function of the length of the chain $G(N) \approx G_{c} \exp (-\beta N)$; for more details, see Sec. VI.C.2. Here the inverse of $G_{c}$ defines the contact resistance associated with left and right anchors, while the exponent $\beta$ describes the attenuation coefficient of the transmission per wire unit. For alkanes, the reported literature values range from $\beta=0.8$ to 1.1 with few exceptions (Tewari, 2018). ${ }^{20}$ For silanes and germanes smaller values have been found, $\beta=0.75$,

\footnotetext{
${ }^{20}$ There are two established ways of expressing the exponential dependence: (1) by the number of monomers added, which is the most unambiguous, indicated here by the symbol $\beta$, and (2) by the length in nanometers (or angstroms) per monomer, for which we reserve the symbol $\beta_{\ell}$. The latter is useful when we are interested in the resistance dependence on length, but it requires a conversion step. For alkane chains the $\mathrm{C}-\mathrm{C}$ bond length is typically used in the conversion. However, the carbon wire backbone is not straight. Instead, one could use the $\mathrm{C}-\mathrm{C}$ bond length projected along the wire axis, but this would be a property that depends on the state of stretching of the wire. When adhering to widely adopted practices, we express $\beta_{\ell}$ as a function of length using a straight $\mathrm{C}-\mathrm{C}$ bond length of $1.26 \AA$, the decay constants are $0.63-0.87 \AA^{-1}$
}

compared to $\beta=0.94$ for alkanes obtained under similar experimental conditions ( $\mathrm{Su}$ et al., 2015).

In this spirit, the length dependence of the conductance for many other molecular wires has been analyzed in terms of an effective exponent $\beta$. We argue later that such exponential dependence is often not properly justified.

\section{Basic concepts}

For a convenient discussion of the experimental observations, we briefly recall the relevant theoretical concepts (Gunasekaran et al., 2018). Long molecular wires that are built out of a single repetitive unit can be categorized in terms of electronic band-structure theory. In particular, molecular orbitals take the form of Bloch states with an associated crystal momentum $k$. The $k$-state classification is highly useful even for wires with a finite length $N$. This is because similar to the "particle-in-the-box" problem, the electronic properties of the molecule can be obtained from the properties of the crystalline wire by imposing selection rules on "allowed" $k$ space momenta. As a consequence, there is a length $N>N^{*}$ beyond which the molecular wire exhibits properties that fully reflect the insulating limit $N \rightarrow \infty$, as is applicable for alkane wires. In particular, in this asymptotic limit the HOMOLUMO gap $\Delta_{N}$ approaches the bulk gap $\Delta_{\text {bulk }}$ and the attenuation

$$
\beta:=-\frac{d \log G(N)}{d N}
$$

takes a constant value $\beta_{\infty}$. Depending on the molecule and its anchor groups, the asymptotic regime $N>N^{*}$ may be challenging to reach in experiments; even small but systematic deviations of $\beta$ from a constant may indicate that this regime is still far away.

To rationalize how $\beta_{\infty}$ relates to the band structure of the infinite wire we recall that $\epsilon_{H}<\epsilon_{F}<\epsilon_{L}$, where $\epsilon_{H, L}$ approach the top of the valence band or bottom of the conduction band at $N>N^{*}$, respectively. We recognize this as a tunneling problem where the height of the barrier $\delta$ is approximately given by the energy difference of $\epsilon_{F}$ to either $\epsilon_{H}$ or $\epsilon_{L}$, depending on which is closer.

To relate $\delta$ to $\beta_{\infty}$, we recall a result for the exponent familiar from the one-dimensional tunneling problem $\beta \sim \sqrt{2 m\left(V_{\text {barrier }}-\epsilon_{F}\right)} / \hbar$. In the case of the molecular barrier, the effective mass $m$ follows directly from the curvature of the band structure around the band edges. For instance, in the case where the LUMO is close to $\epsilon_{F}$, we have an implicit definition $\delta \approx-\varepsilon_{\text {cond }}(i \kappa)$ and $\beta_{\infty}=\kappa a$. The formula involves the band structure $\varepsilon_{\text {cond }}(k)$ of the conduction band (counted from bottom of the band) and the crystalline lattice constant $a$. The expression is further motivated in complex band-structure theory (Reuter, 2017). A formula that interpolates between the two limiting cases where $\epsilon_{F}$ is close to either one of the frontier orbitals was derived by Joachim and Magoga (2002).

We emphasize a basic consequence of these considerations that often is not fully appreciated: in the asymptotic limit, the exponent $\beta_{\infty}$ is a property of the molecule alone. It does not reflect any aspects of the molecular junction other than the 
location of the Fermi level. In particular, $\beta_{\infty}$ does not depend on the choice of the anchor groups. ${ }^{21}$

With an eye on experiments, we note that our discussion focuses on phase-coherent transport. If the wire length increases beyond the phase-coherence length $N_{\text {coh }}$, the exponential decay of the conductance will give way to a weaker decay that reflects an incoherent and strongly temperature dependent dynamics; see Sec. V.A.4.

\section{Conjugation and metallicity}

As is well known in organic chemistry, the properties of conjugated molecular wires differ strongly from those of carbon chains with all saturated bonds, as exemplified by the alkanes. Conjugated molecular wires are characterized by a path of alternating single and double $\mathrm{C}-\mathrm{C}$ bonds as a result of dangling $p$ orbitals on each of the carbons. Every unit cell contributes a single electron to the conduction band. Therefore, conjugated wires generically exhibit a metallic behavior, i.e., a HOMO-LUMO gap that vanishes like $1 / N$ in the asymptotic limit. Exceptions occur in the presence of strong interactions (Schmitteckert et al., 2017) or, if instabilities interfere, such as the Peierls transition in polyacetylene (Heeger et al., 1988). In the absence of a band gap, the conditions for a purely exponential length dependence of the conductance of a molecular wire are not fulfilled.

Effects of breaking the conjugation were discussed in Sec. IV.B.4. In essence, breaking the conjugation, even at only a single point along the wire, introduces a strong scattering center and therefore leads to a reduction of the conductance, as illustrated for single-molecule measurements on oligo( $p$-phenylene ethynylene) (OPE) derivatives (Kaliginedi et al., 2012).

\section{Length dependence for conjugated wires}

In several series of experiments, small attenuation constants $\beta_{\ell}$ for molecular wires have been reported. For instance, for the OPE molecular wires just mentioned (Liu et al., 2008; Kaliginedi et al., 2012) values for $\beta_{\ell}$ of 0.34 and $0.21 \AA^{-1}$ were found. Still smaller values have been obtained for oligothiophenes $\beta_{\ell}=0.1 \AA^{-1}$ (Yamada et al., 2008), oligoyne $\beta_{\ell}=0.06 \AA^{-1}$ (Wang et al., 2009), and oligoporphyrins $\beta_{\ell}=0.04 \AA^{-1}$ (Sedghi et al., 2011). The small $\beta_{\ell}$ values have been used for arguing that these wires are in the metallic regime. We add three warnings.

First, we argued earlier that one can equally well represent the decay constant $\beta$ in terms of the decay per monomer. The attenuation constants in units per monomer for OPE molecular wires are $\beta=1.5$ (Liu et al., 2008) and 2.35

\footnotetext{
${ }^{21}$ Stuyver, Blotwijk et al. (2017) considered the possibility that the anchor groups affect $\beta_{\infty}$ by shifting the Fermi energy $\epsilon_{F}$. We point out here that a single molecule attached to metallic substrates cannot modify $\epsilon_{F}$. Such an effect can take place only in the presence of a finite concentration of molecules, as they occur in self-assembled monolayers. In this case, the surface dipole of the substrate, and hence $\epsilon_{F}$, can be modified by an amount that scales with the concentration of molecules per surface area. Ab initio calculations can illustrate this effect (Obersteiner et al., 2017).
}

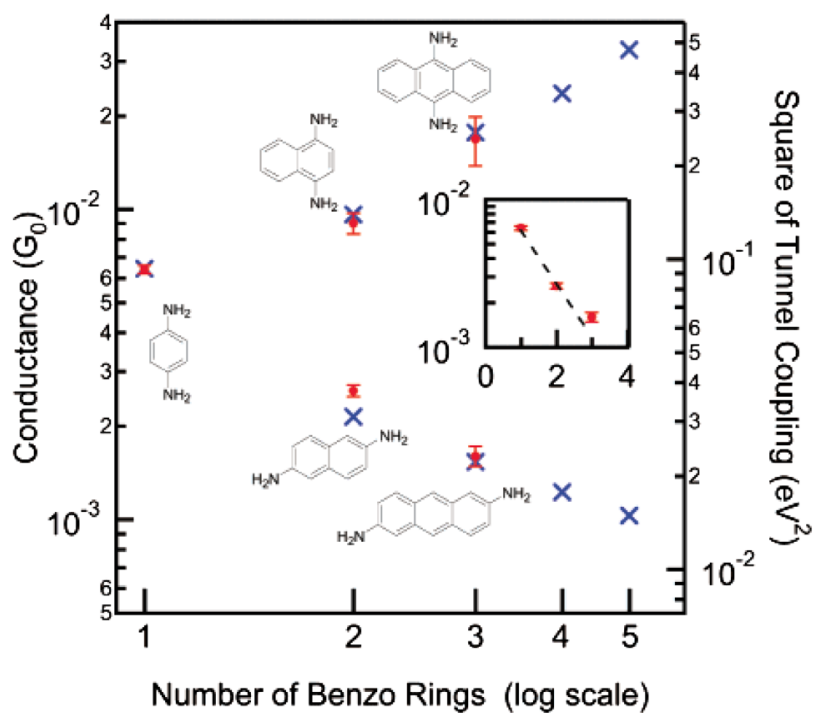

FIG. 15. Conductance measured for a series of diaminoacenes as a function of the number $N$ of benzo rings (red dots, left axis). When measured in the transverse direction (upper data points) the conductance increases with $N$, while when measured longitudinally the conductance decreases. The log-linear plot in the inset shows that the latter is not a simple exponential decrease. The two trends are reproduced by calculations for the square of the tunnel coupling (blue crosses, right scale). From Quinn et al., 2007.

(Kaliginedi et al., 2012). For oligothiophenes $\beta=0.42$ (Yamada et al., 2008), for oligoyne $\beta=0.18$ (Wang et al., 2009), and for oligoporphyrins $\beta=0.55$ (Sedghi et al., 2011), and for oligoacenes (Quinn et al., 2007) in the longitudinal direction $\beta=0.7$. This shows that most of these numbers are comparable to those typical of insulators.

Second, the series of molecules considered in those works is small, in nearly all cases covering only three points. From these three points one cannot rigorously distinguish an exponential dependence from a dependence on inverse length. The exponential dependence would be consistent with a finite energy gap in the $N \rightarrow \infty$ limit, meaning that the wire is an insulator. A decrease of conductance as $1 / N$ is consistent with a metallic wire in the hopping regime.

Third, the widespread practice of extracting a simple exponent from the decay of the conductance is at variance with the expected behavior for phase-coherent metallic wires, such as those given in the forthcoming theoretical considerations.

Figure 15 reproduces experimental data obtained by Quinn et al. (2007) showing how the transmission of an oligoacene evolves with increasing length, $N$, depending on the placement of the anchor groups. The conductance decreases when measured along the wire length, but the trend is not purely exponential, as illustrated in the inset of the figure. In contrast, the conductance measured in the transverse direction increases with length, as shown for the upper series in the plot. We take this latter observation as a strong indication that the conductance in this type of wires can be thought of as phase coherent. For describing length dependence of phase-coherent transport in metallic molecular wires we invoke basic scaling arguments. 
Theoretical considerations.-We follow Yelin et al. (2016) and focus on the situation of well-separated levels where the conductance is dominated by a single orbital only. In this case, the transmission $\mathcal{T}(E)$ can be approximated by three parameters $\Gamma_{\mathcal{L}, \mathcal{R}}(N)$ and the level position relative to $\epsilon_{F}, \epsilon(N)$,

$$
\mathcal{T}(E)=\frac{\Gamma_{\mathcal{L}} \Gamma_{\mathcal{R}}}{(E-\epsilon)^{2}+\left(\Gamma_{\mathcal{L}}+\Gamma_{\mathcal{R}}\right)^{2} / 4} .
$$

Since the band structure typically is analytic near the Fermi energy, we make an expansion

$$
\epsilon(N)=\epsilon_{0}+\epsilon_{1} / N+\epsilon_{2} / N^{2}+\cdots .
$$

As written here, the expansion applies to metals and insulators. In the latter case, $\epsilon_{0}$ accounts for the offset between the Fermi energy and the closest band edge. For the case of metallic wires, which we consider here, $\epsilon_{0}=0$. Equation (51) formalizes the idea that the spectrum of finite-length wires derives from the band structure of the bulk by imposing $k$ space selection rules with $\delta k \sim 2 \pi / N$ for neighboring $k$ values. The slope $\epsilon_{1}$ is of the order of the Fermi energy, while the curvature $\epsilon_{2}$ corresponds to an inverse band mass.

Note that $k$-space selection rules are sensitive to boundary effects (Dasgupta et al., 2012; Korytár et al., 2014). For example, electrophilic anchor groups can shift a LUMO-based transport resonance closer to $\epsilon_{F}$. Therefore, in general the expansion coefficients comprise information about the molecular wire and its anchoring.

Concerning the level broadening, we observe that the wire's Bloch states extend homogeneously over the wire. Hence, asymptotically their overlap with the contact sites, which connect to the electrodes, is inversely proportional to the length of the wire:

$$
\Gamma_{\mathcal{L}, \mathcal{R}} \approx c_{\mathcal{L}, \mathcal{R}} / N
$$

Collecting formulas and inserting them into Eq. (50), we obtain

$$
\mathcal{T}\left(\epsilon_{0}\right) \approx \frac{\mathcal{T}_{\infty}}{1+2\left(\epsilon_{1} / \epsilon_{2}\right) N_{c}^{2} / N+\left(N_{c} / N\right)^{2}},
$$

where $\mathcal{T}_{\infty}=4 c_{\mathcal{L}} c_{\mathcal{R}} /\left[4 \epsilon_{1}^{2}+\left(c_{\mathcal{L}}+c_{\mathcal{R}}\right)^{2}\right] \quad$ and $\quad N_{c}^{2}=4 \epsilon_{2}^{2} /$ $\left[4 \epsilon_{1}^{2}+\left(c_{\mathcal{L}}+c_{\mathcal{R}}\right)^{2}\right]$.

As is readily seen from this result, if $\epsilon_{1}$ and $\epsilon_{2}$ have the same sign, the conductance increases monotonically approaching the asymptotic value $\mathcal{T}_{\infty}$ from below. In the opposite situation in which $\epsilon_{1}$ and $\epsilon_{2}$ have opposing signs, the evolution of the transmission can be nonmonotonic. It moves first through a maximum of $\mathcal{T}_{\text {max }}=4 c_{\mathcal{L}} c_{\mathcal{R}} /\left(c_{\mathcal{L}}+c_{\mathcal{R}}\right)^{2}$ at $N_{\text {max }}=\left|\epsilon_{1} / \epsilon_{2}\right|$ before approaching the asymptotic value from above.

Application to experiment.-The concepts developed earlier have been successfully applied for understanding the evolution of the conductance of oligoacene wires under conditions where these wires were attached directly to the metal leads $(\mathrm{Ag}$ or $\mathrm{Pt})$ without employing anchor groups (Yelin et al., 2016). In the case of Ag leads, the molecular level spacing is much larger than the level broadening and the transport is entirely dominated by the LUMO's, as shown by $a b$ initio transmissions in Fig. 16. The exception is benzene

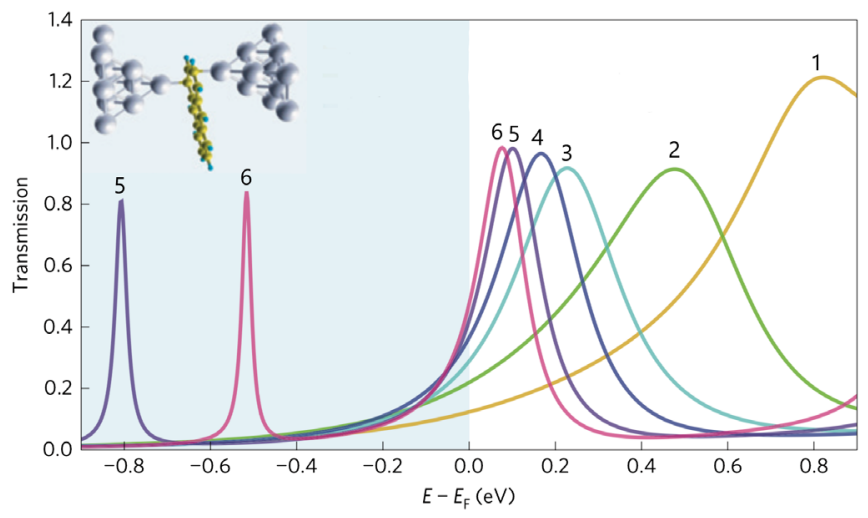

FIG. 16. Transmission resonances of oligoacenes directly bound to Ag contacts for geometries as exemplified in the inset for anthracene. This provides an example of the evolution of the transmission resonances that exhibit growth of the transmission with increasing molecular length. The numbers above the curves indicate the numbers of carbon rings: benzene (1), naphthalene (2), anthracene (3), tetracene (4), pentacene (5), and hexacene (6). From Yelin et al., 2016.

$(N=1)$ with two transport-active orbitals. For $N>1$, the resonances are approximately Lorentzian, and the scaling arguments of Eqs. (51)-(53) apply. Both the peak width and the peak position decrease with $N$, and saturation is observed.

Returning to Fig. 15, where amine anchors are used in transport measurements for a series of acenes, we analyze the trends observed there, thus giving a fresh example to demonstrate how useful these concepts can be. In our analysis, we assume that the level broadening is less sensitive to the position of the anchor groups, transverse or longitudinal, than to the level spacing, which we refer to as $\epsilon^{\text {trans }}(N)$ and $\epsilon^{\text {long }}(N)$, respectively. Our assumption implies that the expansion coefficients $c_{\mathcal{L}, \mathcal{R}}$ in Eq. (52) for the transversal and longitudinal placing of the anchor groups are roughly the same. ${ }^{22}$ Furthermore, from Yelin et al. (2016) we adopt the result that transport will be LUMO based, so $\epsilon_{1}$ is positive for both cases, longitudinal and transversal. Since the asymptotic values of the conductance seen in Fig. 15 are significantly different $\mathcal{T}_{\infty}^{\text {long }} \ll \mathcal{T}_{\infty}^{\text {trans }}$, we conclude that $\epsilon_{1}^{\text {trans }} \ll \epsilon_{1}^{\text {long }}$. This finding is understood as follows: owing to the electrophilic character of nitrogen, the amino-based anchor groups pull the LUMO level closer to $\epsilon_{F}$. If the anchor groups are far apart (longitudinal) they compete when attracting molecule-based charge, while they cooperate if they are close (transverse). Therefore, the LUMO is expected to be closer to $\epsilon_{F}$ in the latter case and the transmission is enhanced. The difference between $\epsilon_{1}^{\text {trans }}$ and $\epsilon_{1}^{\text {long }}$ is further amplified by image charges in the leads. These have the effect of reducing the HOMOLUMO gap, and this reduction grows strongly when the electrodes are closer to the molecule.

\footnotetext{
${ }^{22}$ We recall that in tight-binding descriptions the level broadening is given by $|t|^{2} \rho_{\text {Fermi }}$, where $t$ denotes a hopping matrix element between the molecule and the reservoir and $\rho_{\text {Fermi }}$ denotes the density of states on the reservoir contact site. One would expect that neither $t$ nor $\rho_{\text {Fermi }}$ are sensitive to the placement of the contacts, transverse or longitudinal.
} 
We observe that in the limit of short wires there is no pronounced difference between the two cases, $\mathcal{T}^{\text {long }}=\mathcal{T}^{\text {trans }}$ for $N \downarrow 1$. This matching condition can be satisfied if the large contribution of the longitudinal case $\epsilon_{1}^{\text {long }}$ is partially canceled by the second term $\epsilon_{2}^{\text {long }}$ in Eq. (51). To facilitate this, the two coefficients should have opposite signs $\epsilon_{1}^{\text {long }} \approx-\epsilon_{2}^{\text {long }}$. Note that there is no such expectation in the transverse case. Correspondingly, Eq. (53) predicts an asymptotic decay of the transmission in the longitudinal case (crossover length $N_{\max }=\left|\epsilon_{1} / \epsilon_{2}\right| \approx 1$ ) and an increase in the transverse case, qualitatively consistent with the experiment shown in Fig. 15.

\section{Incoherent transport limit}

Several groups have reported a transition from exponential decay of the conductance with wire length $N$ to a slower, nearly linear dependence above a certain value of $N$. Examples were given by Choi, Kim, and Frisbie (2008) and Choi et al. (2010) for oligonaphthalenefluoreneimine up to $N=10$, and by Hines et al. (2010) for conjugated molecular wires up to $9.4 \mathrm{~nm}$ in length. While the phenomenon is attributed to a crossover from coherent tunneling to thermally activated hopping, the deeply inelastic regime has not yet been experimentally investigated in detail. One expects molecular-type Bloch states, which originally extend over the full length of the molecule, to become localized. Several mechanisms are conceivable that can drive the process. One possibility is a spontaneous breaking of translation invariance due to the formation of a polaron. The process could be effective in wires, which have a soft molecular backbone, or in strongly polarizable environments. Alternatively, a thermal activation of deformations of the molecule, notably ring rotations (see Sec. VI) could be involved. This suggests that the crossover is strongly temperature dependent, as has been observed (Choi et al., 2010; Hines et al., 2010; Smith et al., 2015).

\section{B. Conductance as a function of molecular conformation}

The breaking of conjugation in molecular wires has been studied systematically by designing a series of molecular wires for which the neighboring phenyl groups have a rotation fixed by suitable choice of side groups. For a series of biphenyl-based molecules with varying degrees of sterically constrained rotation of the two phenyl rings, Venkataraman, Klare, Nuckolls et al. (2006) found that the conductance for this series decreases proportionally to $\cos ^{2}(\vartheta)$, with $\vartheta$ the angle between the two rings; see Fig. 17. This is the dependence expected to result from the overlap of the $\pi$ orbital systems on the two rings. This was confirmed by other methods of constraining the ring rotations in the study by Mishchenko et al. (2010).

The observed dependence $G=a \cos ^{2}(\vartheta)$ agrees with detailed $a b$ initio computations (Hybertsen et al., 2008; Mishchenko et al., 2010). The twist-angle dependence can be reproduced by a simple two-site model, where the two sites represent the two phenyl rings (Mishchenko et al., 2010). As long as the molecular levels are far removed from the Fermi energy on the scale of the energy level broadening and the intersite coupling, the angle dependence is purely given by
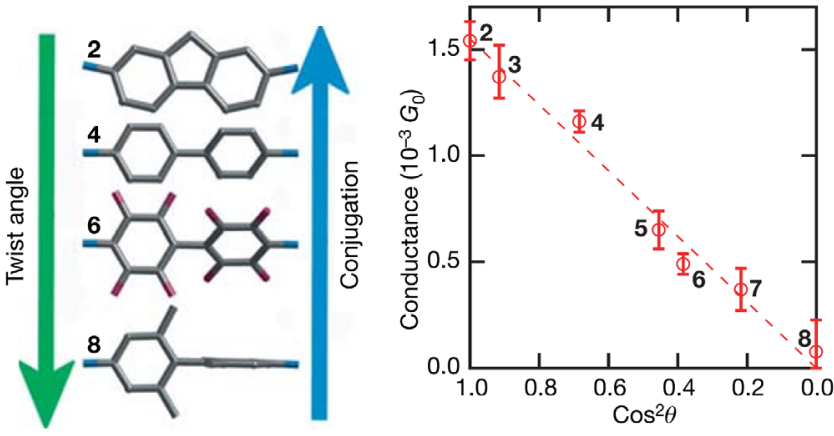

FIG. 17. Conductance as a function of the twist angle between two phenyl rings. (Left panel) By design, steric interactions constrain the twist angles between the two phenyl rings of a series of molecules. The smaller the angle $\theta$ the larger the overlap of the wave functions (the conjugation) between the two ring sections. (Right panel) The measured conductance follows the expected $\cos ^{2} \theta$ dependence. Adapted from Venkataraman, Klare, Nuckolls et al., 2006.

$\cos ^{2}(\vartheta)$. When the levels move closer to resonance, terms in $\cos ^{4}(\vartheta)$ appear, but due to cancellations they remain small.

Note that the slope $a$ obtained from fitting the DFT results is 3 orders of magnitude higher than the slope obtained from the experimental data because all conductance values are so much lower in experiment. One of the possible explanations offered by Mishchenko et al. (2010) is that the transport under the experimental conditions is not fully coherent. Even under such conditions, the $\cos ^{2}(\vartheta)$ dependence is robust and survives.

\section{Anchor groups}

The physical properties of a molecular junction depend significantly on the atomic details of how the molecule binds to the electrodes. For this reason the optimal choice of the anchor group has been an important research topic in the field from the beginning. The role of the molecule-electrode interface was covered in a review by $\mathrm{Su}$ et al. (2016), highlighting the relevant chemical principles. Further discussions, and a list of anchors that have been tested, are given by Jia and Guo (2013) and Hybertsen and Venkataraman (2016).

One may identify three important roles that anchor groups have in determining the properties of single-molecule junctions. First, the end groups need to provide mechanical anchoring of the molecule to the metal leads, in the sense of bonds that resist breaking by thermal or mechanical agitation. Second, they should provide electrical contact between the metal leads and the core of the molecule. In many cases, the goal has been to achieve nearly unimpeded transmission of electrons from the metal to the core of the molecule such that the properties of the latter dominate the junction properties. Finally, the anchor groups influence the alignment of the frontier orbitals of the core of the molecule with the Fermi level in the leads.

\section{Thiol-based anchoring groups}

In most experiments (Scheer and Cuevas, 2017), $\mathrm{Au}$ electrodes have been used in combination with thiol anchors. This prominent role deserves special mention here, and we 
also argue later that the nature of the bonding is still a matter of debate.

$\mathrm{Au}$ surfaces are natural candidates because they exhibit a low tendency toward contamination. The choice for thiol linkers is motivated mostly by the fact that these make strong bonds to $\mathrm{Au}$, promoting a single point of contact between the molecule and each of the leads. The mechanical coupling they provide is strong enough for producing frequently appearing plateaus in conductance-breaking traces, and the electronic coupling is sufficient for the properties of the core of the molecule to be observable. They have the drawback that thiols are easily oxidized, resulting in polymerization by the formation of S-S bonds between the molecules. This can be avoided by replacing the $\mathrm{H}$ in the thiol group by a protection group, often chosen to be an acetyl group. This protection group slows down the immobilization kinetics of the molecules (Elbing et al., 2005). The protection group can be removed during exposure of the Au surface to the molecular solution by adding $\mathrm{NH}_{4} \mathrm{OH}$ (Tour et al., 1995) or tetrabutylammonium hydroxide (Grunder et al., 2007) as a deprotection agent. Alternatively, the acetyl-protected molecules can directly adsorb on the Au electrodes without the use of a deprotection agent. However, it has been shown (Tour et al., 1995) that for the direct adsorption of the thioacetyl containing molecules and formation of self-assembled monolayers a larger concentration of molecules is required.

For all work on thiol anchors, an important issue was raised by Stokbro et al. (2003), namely, whether in the interaction between the thiol group and the Au surface the hydrogen atom actually splits off. Computations suggest that the thiol bond (with the $\mathrm{S}-\mathrm{H}$ bond intact) and the thiolate bond to the $\mathrm{Au}$ surface (with the $\mathrm{H}$ removed) are nearly equivalent in energy, and there is experimental evidence that both may occur (Rzeznicka et al., 2005). Recent experimental evidence based on STM break junctions for single molecules by Inkpen et al. (2018) showed that the formation of thiol or thiolate bonds sensitively depends on the preparation conditions.

\section{The role of mechanical coupling}

One may be inclined to select anchor groups that provide the strongest mechanical coupling. On the other hand, arguments have been put forward that optimizing anchor groups toward strong mechanical coupling may not be favorable for producing clear signatures of molecular conductance in conductance histograms. In STM break-junction experiments, the conductance distribution (peak width) obtained with strongly binding thiol linkers was found to be much wider than that for amine anchors that have smaller binding strength (Venkataraman, Klare, Tam et al., 2006). The interpretation offered is based on the flexibility of the Au-amine bond, which leaves the arrangement of the Au surface atoms unaffected. In contrast, the $\mathrm{Au}-\mathrm{S}$ bond is stronger than a $\mathrm{Au}-\mathrm{Au}$ bond resulting in restructuring of the metal electrodes during stretching of the contacts. Consequently, conductance histograms are based on many metal-electrode surface configurations; for reviews, see Li and Kosov (2007) and Hybertsen and Venkataraman (2016).

Surprisingly, the opposite result was found for MCBJ break junctions by Martin, Ding, van der Zant, and van Ruitenbeek
(2008): the thiol-coupled molecules produced a stronger and sharper signature in the conductance histograms than their amine-coupled counterparts. In a study comparing results for different anchoring groups, Chen et al. (2006) found no major difference between thiol, amine or carboxylic acid anchoring of alkanes, except for minor shifts in the conductance peak position. As suggested by Martin, Ding, van der Zant, and van Ruitenbeek (2008), the outcome of the experiments may depend sensitively on the experimental conditions. The experiments by Venkataraman, Klare, Tam et al. (2006) were performed in solution, where bond breaking may be followed by spontaneous reforming of bonds. This may enhance the signature for a weak bond, such as the amine bond, in the conductance histograms. When performing experiments under vacuum with sparse surface coverage, on the other hand, once a bond is broken it cannot be reformed spontaneously. In such experiments a stronger bond, such as a thiol bond, may be preferable.

Taking the last point one step further, multidentate bonds have been investigated. One may reason that multiple anchoring points at each anchoring site could lead to the molecule imposing the structure of the metal leads, which would suppress the variability in the conductance histograms. Such multidentate bonds have been explored in the forms of carbodithioate $\left(-\mathrm{CS}_{2} \mathrm{H}\right)$ groups (Tivanski et al., 2005; Xing et al., 2010) and dithiocarbamate $\left(-\mathrm{NCS}_{2} \mathrm{H}\right)$ groups (von Wrochem et al., 2010). A systematic comparison of the conductance histograms with those of other anchoring groups has not yet been made.

\section{Anchor transparency and gateway states}

The formation of electrical contact between the molecule and the electrodes can be discussed in terms of the hybridization of the orbitals on the molecule with the surface states of the electrodes; see Sec. III.B. The degree of hybridization is determined by the amount of overlap that the anchor-group orbitals have with the foremost electrode atom(s). Therefore, the classification of anchor groups follows largely the atomic orbital theory of the chemical bond. For instance, one distinguishes donor-acceptor-type anchors from covalent anchors (Su et al., 2016). The setting of the anchor and the associated hybridization of orbitals follows the local rules of optimizing atomic overlaps.

In many cases, anchoring orbitals do not strongly hybridize with the frontier orbitals of the molecular backbone and lie at much lower energy. As a result, the effect of the anchor groups on the transmission of a molecular wire can be accounted for by a contact resistance. The picture is that the anchor resembles a tunneling barrier for the charge carriers that is characterized by only a single parameter, its transparency, which is assumed to be roughly independent of the energy of the incoming particle. This picture is applicable to some of the long insulating wires discussed previously, whose conductance is captured in the asymptotic expression $G=G_{c} e^{-\beta N}$, where $N$ denotes the length of the wire in units of its monomer and $G_{c}$ denotes the limiting value due to the contacts; see Sec. V.A.

However, it has been noticed that more complicated situations can arise, when the atomic orbitals of the anchor 
groups lie closer to the Fermi energy than the frontier orbitals of the molecular wire (Li et al., 2008). Specifically, consider the case of an alkane wire with a thiolate bond. The sulfur atom, when binding to a $\mathrm{Au}$ electrode, exhibits a localized orbital with an energy situated in the band gap of the alkane wire. This in-gap state ["contact" state (Li et al., 2008) or "gateway" state (Vázquez et al., 2012)] is associated with a broad transport resonance; it can dominate the transmission of the shorter alkane chains (Li et al., 2008). Gateway states have been observed in various theoretical studies (Brooke et al., 2015; Hüser and Solomon, 2015) and need to be accounted for when quantitatively evaluating experiments on quantum interference (Vázquez et al., 2012).

In the presence of gateway states, the asymptotic behavior of the conductance $G \sim e^{-\beta N}$ sets in only at large $N$ when, technically speaking, the passage through the insulating wire dominates the tunneling action. Only in this limit is the conductance truly exponential in the length, and the gap exponent $\beta$ is a property of the band structure of the long wire, i.e., independent of the contact arrangement. In their experimental work, Sangtarash et al. (2018) observed in alkane wires with an extra aromatic center unit an approximately exponential decay $G(N) \approx e^{-\beta^{\prime} N}$ with an effective exponent $\beta^{\prime}$ that is considerably smaller than $\beta$. Sangtarash et al. explained their observation by invoking in-gap (gateway) states. From our perspective one would expect the effective exponent $\beta^{\prime}$ to characterize a preasymptotic regime that crosses over into a steeper decay at larger $N$.

The concepts discussed in this section are illustrated in Fig. 18 for an alkanedithiol (ADT) gold junction (Li et al., 2008). Two molecular orbitals dominate the length dependence of the conductance. At $-2.4 \mathrm{eV}$, there is the $\mathrm{HOMO}^{*}$ of ADT, which is the HOMO of the alkanes and the backbone state. Its weight at the Fermi energy decreases exponentially

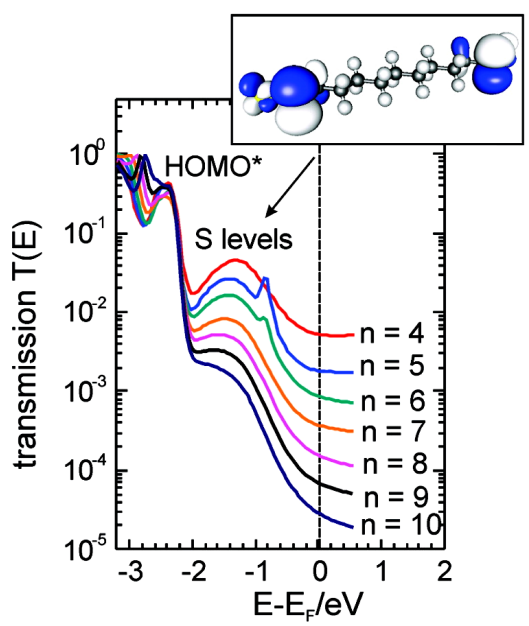

FIG. 18. Transmission functions of Au-alkanedithiol-Au series, where the number of carbons is denoted by $n$. The length dependence of the conductance is determined by the spatially delocalized $\mathrm{HOMO}^{*}$ resonance and a broad resonance around $E=-1.5 \mathrm{eV}$, which corresponds to the gateway state (HOMO, "S level"). (Inset) The wave function isosurface of the HOMO and the ball-and-stick model with carbons (black), hydrogens (white), and sulphurs at the ends (yellow). From Li et al., 2008. with length, which is consistent with the tunneling picture. The resonance at $-1.5 \mathrm{eV}$ is the HOMO of ADT, which is the gateway state, not present for bare alkanes. Its width is almost length independent due to the orbital's location being close to one electrode, its position approaches $\mathrm{HOMO}^{*}$ and its weight drops exponentially due to its localized nature.

\section{Direct metal-molecule coupling}

It is possible to form direct links between metal electrodes and the carbon backbone of molecules. This can be achieved by interaction of organic molecules without anchoring groups with reactive metal electrodes such as $\mathrm{Pt}$ under cryogenic vacuum conditions (Kiguchi et al., 2008; Yelin et al., 2016). The coupling to $\mathrm{Pt}$ electrodes results in conductance even above $1 G_{0}$ because multiple conductance channels are participating in transport. Alternatively, coupling reactions have been exploited based on trimethyl-tin $\left(-\mathrm{SnMe}_{3}\right)$ terminations. Upon exposing the molecules to Au surfaces, the Sn terminal groups are split off and replaced by direct $\mathrm{Au}-\mathrm{C}$ bonds (Cheng et al., 2011). Such direct Au-C coupled junctions have transmissions that exceed the ones for anchor-group coupled molecules and can even produce nearly perfect transmission (Chen et al., 2011).

Further methods for direct coupling of $\mathrm{Au}$ to $\mathrm{C}$ exploit $\mathrm{C} \equiv \mathrm{C}$ triple bonded end groups, as described by Hong et al. (2012) and Olavarria-Contreras et al. (2016). The Au-C $\equiv \mathrm{C}$ coupling leads to $s p$ hybridization, which does not optimally couple the Au $s$ states to the molecular backbone. Indeed, the conductance is lower than for analogous $s p^{3}$-hybridized $\mathrm{Au}-\mathrm{C}$ bonds (Olavarria-Contreras et al., 2016).

Despite the absence of an explicit anchoring group, gateway states appear even for direct $s p^{3}$ hybridized Au-C bonds and can dominate the transmission (Batra et al., 2013; Widawsky et al., 2013). In such cases, the gateway state is formed by a $\mathrm{Au}-\mathrm{C} \sigma$ bonding orbital. This insight is essential for rationalizing the observed combined data for conductance and thermopower for series of molecular wires (Widawsky et al., 2013).

\section{Level alignment}

A nonlocal aspect of molecular junctions concerns the occupation of the junction states: their filling is controlled by the alignment of their energy level with the electrode's work function. The occupation thus depends on certain global properties, like the surface orientation, and on the materials chosen. The partial filling of the frontier orbitals decides between particle (LUMO-based) versus hole (HOMO-based) transport. Since the filling depends on a combination of local and global aspects of the junction, precise rules for transport properties based on the nature of the anchor groups alone are difficult to establish. Su et al. (2016) proposed as a rule of thumb that dative anchor groups tend to come with hole transport, while electron-withdrawing groups favor particle transport.

\section{Quantum interference}

After introducing the theory of QI in molecular junctions in Sec. IV.B, we review key measurements here that demonstrate 


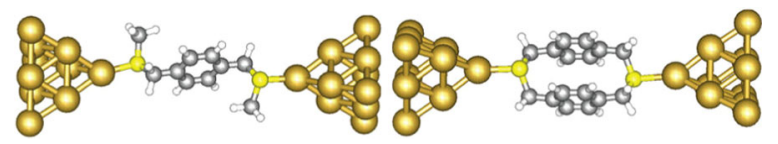

FIG. 19. One-path and two-path molecules used for demonstrating constructive quantum interference in the experiment by Vázquez et al. (2012).

the experimental significance of these concepts. As a preparatory remark, we emphasize that experiments on QI in molecular junctions are necessarily somewhat less direct than those using microfabricated mesoscopic devices. For the latter, it is relatively straightforward to manipulate QI, e.g., by application of magnetic fields. This route is not open for molecular junctions because the field strength necessary to achieve a measurable effect is not practical. Therefore, for the systems of interest here one proceeds via a combination of measurement and theoretical analysis. This point given, a remarkable number of experimental investigations have been performed in recent years that all support the existence of strong QI effects in molecular electronics.

An early piece of indirect evidence of QI was reported by Ballmann et al. (2012), as mentioned in Sec. IV.B. They explained the observed increase of the conductance with temperature as a result of lifting the destructive QI by molecular vibrations. In another experiment, Vázquez et al. (2012) were able to perform a two-path experiment employing a special molecular design, as shown in Fig. 19. Ideally, one expects the conductance to increase by a factor of 4 when adding a second parallel channel. In the measurement, a factor of 3 has been observed, which indeed significantly exceeds the classical limit of 2.

Particularly strong effects of QI occur in molecules that exhibit a Fano-type antiresonance. Motivated by theoretical considerations (see Sec. IV.B), molecules have been synthesized that exhibit the predicted conductance suppression. For example, Guédon et al. (2012) observed that the conductance through an anthraquinone unit is strongly suppressed relative to anthracene; see Fig. 20. By combining DFT and tight-binding calculations, Guédon et al. argued that this effect results from destructive QI; see Fig. 21 for computed transmissions.

In a further experiment by Garner et al. (2018) a bicyclo [2.2.2] octasilane moiety was employed. The QI-induced conduction suppression was so strong that the transmission fell below the vacuum value associated with the gap deprived of its molecular bridge, which represents a "single-molecule insulator."

Signatures of QI are pronounced when $\pi$-type binding dominates the most transmitting states. Then, moving one of the contacts, e.g., the drain, from one atom to a neighboring atom strongly affects the transmission. In the context of alternating hydrocarbons, such a contact displacement implies that in one situation the contacts couple to the same sublattice, while in the other they couple to different sublattices. The corresponding conductance change is readily explained in terms of the concepts introduced in Sec. IV.B.3.

The sensitivity of the transmission to shifts of the contact position has been investigated from early on. For instance,
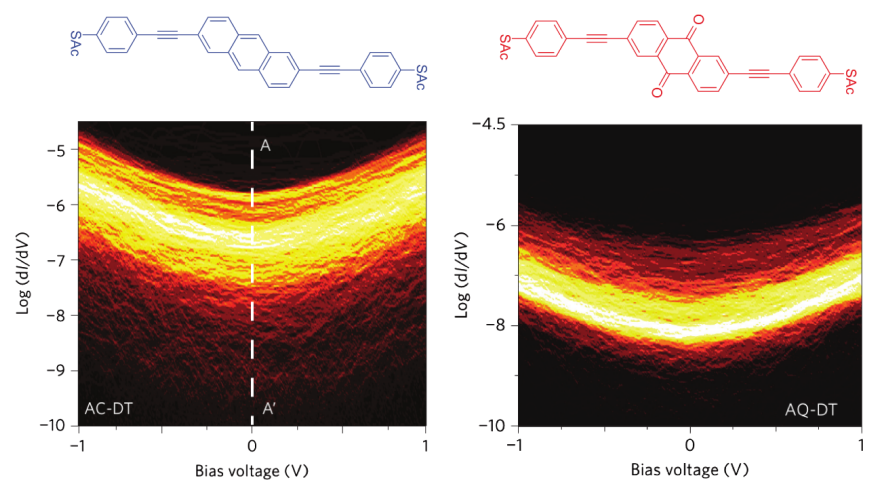

FIG. 20. Conducting-tip AFM measurements of (left panel) the conductance of linearly (anthracene-based) and (right panel) cross-conjugated (anthraquinone-based) molecules. Two-dimensional conductance histograms are shown, constructed by logarithmic binning of $d I / d V$ in units $\Omega^{-1}$ versus bias voltage. The color scale indicates the number of counts, ranging from black $(0$ counts) to white ( $>40$ counts). Destructive QI suppresses transport in cross-conjugated molecules (right panel). Adapted from Guédon et al., 2012.

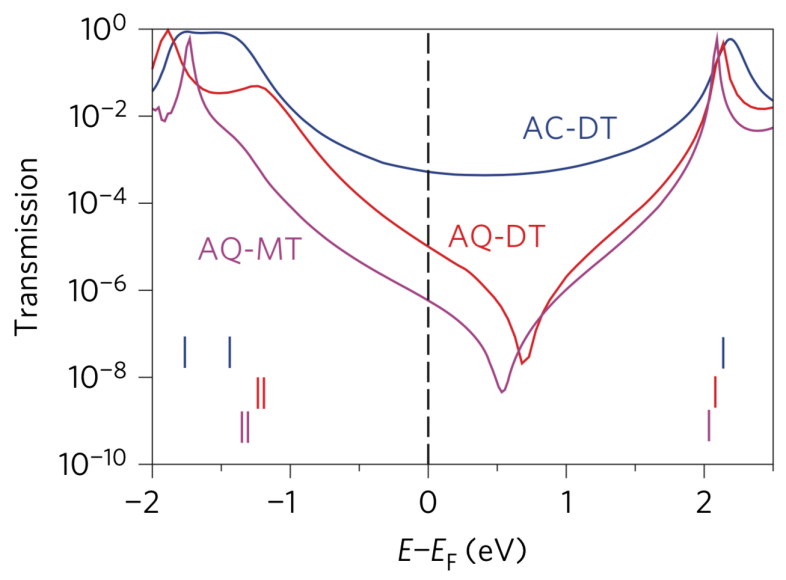

FIG. 21. Transmission curves from $a b$ initio for anthracene (AC) and anthraquinone (AQ) molecules shown in Fig. 20; AQMT (AQ-monothiol) contains only one thiol end group. Vertical bars at the bottom indicate resonance centers of HOMO-1, HOMO, and LUMO for AC-DT (top), AQ-DT (middle), and AQ-MT (bottom). The AC molecules show a pronounced dip between the HOMO and LUMO peaks, which is consistent with rules for bipartite lattices discussed in Sec. IV.B.4. Adapted from Guédon et al., 2012.

Mayor et al. (2003) observed that a benzene ring when used as a linker group with contacts in paraposition (Fig. 22, 1) carries a current much larger than when the contacts are in metaposition (Fig. 22, 2). An intuitive picture put forward at the time to explain the effect was that a contact in metaposition couples to a node of the frontier orbitals, while one in paraposition couples to a maximum. The theoretical concepts presented in Sec. IV.B connecting this observation to the sublattice structure, applied to each terminal phenyl group, provide a broader scope and, in particular, predictive counting rules.

The basic idea of investigating the change of transmission upon varying the contact positions has been followed in subsequent work. Arroyo et al. (2013) considered molecular 


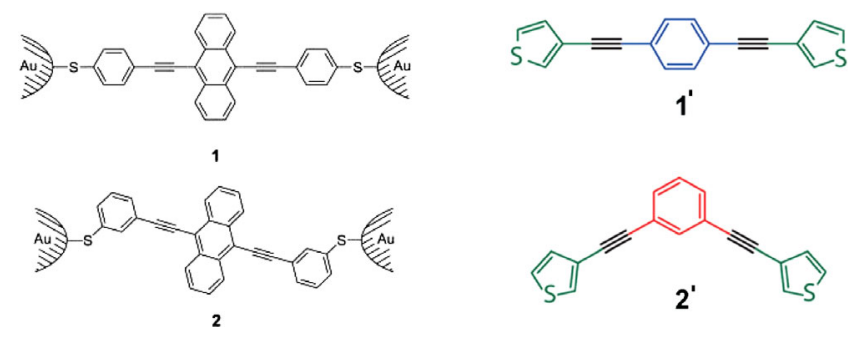

FIG. 22. (Left panel) Molecules investigated by Mayor et al. (2003). Similar species with mixed end groups were investigated by Ballmann et al. (2012). (Right panel) Molecules investigated by Arroyo et al. (2013).

wires having a benzene ring as a center unit; see Fig. 22, 1' and $2^{\prime}$. They confirmed experimentally the expectation that follows from counting rules: the transmission of a single paracoupled benzene ring largely exceeds the one with metacoupling.

Manrique et al. (2015) also confirmed this conclusion. They used in their experiment pyridine rings for anchoring. Their explicit DFT-based calculations showed that the transmissions of pyridine rings in paraposition and orthoposition were similar, while the transmission in metaposition was suppressed. This result once more illustrates that the transmission of benzene rings with contacts coupling to one sublattice only (metaposition) is suppressed in comparison to contacts coupling to both sublattices (paraposition and orthoposition). Further derivatives with benzenethiol end groups and benzene or pyridine centers were studied by Liu et al. (2017). Li et al. (2018) provided further support. Using electrochemical gating they demonstrated that a metaoriented diphenyl benzene structure has a much stronger variation of the transmission with gate voltage than the paracoupled species. An on-off ratio of 200 has been achieved in this way. In a similar experiment performed by Huang et al. (2018), observation of an even larger on-off ratio of 500 was reported.

In addition to molecular rods, more extended graphenelike structures were also investigated, by Sangtarash, Sadeghi, and Lambert (2016). It was also found that in such cases shifting the contact positions to neighboring sites can substantially influence the molecular transmission. Specifically, the typical transmission of the para-type coupling shown in Fig. 23 was found experimentally to exceed the transmission of the metatype contact by a factor of 30 (Geng et al., 2015).

Finally, we address a recent development that points toward a possibility of mechanical control of destructive QI. Stefani et al. (2018) observed a modulation of the conductance of a $\pi$ stacking molecular wire (a [2.2]paracyclophane compound)

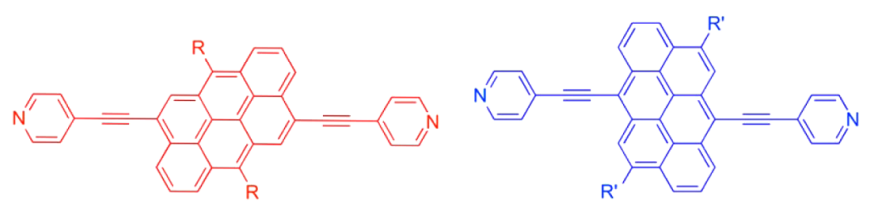

FIG. 23. Molecules with anthanthrene core studied by Geng et al. (2015). within a MCBJ, by an order of magnitude upon pushing or pulling. They interpreted their results as due to a sharp antiresonance in the transmission function that can be relocated with respect to $\epsilon_{F}$ by mechanical manipulation; see also the earlier work by Frisenda et al. (2016).

Mechanical control of destructive QI is also thought to be responsible for the large conductance fluctuations seen in experiments on ferrocene-based molecular wires (CamarasaGómez et al., 2019). In this case, the proposed effect derives from a rotational degree of freedom that can switch the junction's QI from destructive to constructive and back.

\section{E. Electrostatic effects and image charges}

The electrostatic environment for electrons on a molecule changes in various ways when the molecule is placed between two metal leads. For instance, if the Fermi energy of the metal lies outside the HOMO-LUMO gap, charge is transferred between molecule and contact. Thus, a surface dipole is generated, which can substantially alter the work function.

A dramatic demonstration of this effect is obtained when the surface coverage of adsorbents is asymmetric between the two leads. This asymmetry can be obtained when working in a polar solvent into which the molecules of interest have been dissolved, as shown in the experiments by Capozzi et al. (2015). The experiments were performed using the STM-BJ technique for a $\mathrm{Au}$ tip and $\mathrm{Au}$ metal surface and a left-right symmetric oligomer of four units of thiophene-1,1-dioxide having the same anchor groups at both ends. Despite the symmetry of this arrangement, the $I-V$ curves for singlemolecule junctions showed strong asymmetry, with rectification ratios $R_{\mathrm{rr}}:=|I(+V) / I(-V)|$ above 100 . The explanation offered for the observed asymmetry relies entirely on the geometric shape anisotropy of the tip and surface electrodes. The difference in size creates a difference in effective capacitance at the surface, and the largest voltage drop is found at the smallest capacitor, i.e., the tip electrode. The effect disappears in nonpolar solvents.

Apart from a global adjustment of the electrochemical potential the presence of the metal electrodes affects the molecular levels in other ways. Any charge distribution on the molecule creates image charges in the metal electrodes, which results in an electrostatic energy shift of the molecular levels. In break-junction experiments on $\mathrm{Zn}$-porphyrin molecules that resulted in sharp molecular-level resonances in the $I$ - $V$ curves, Perrin et al. (2013) showed that the position of the molecular resonances shifts strongly, over nearly $0.5 \mathrm{eV}$, as a function of the change of distance between the electrodes. The explanation offered for this shift is based on the image-charge potentials, which are sensitive to the distance of the molecule to the metal leads and to its orientation in the junction.

Image charges are expected to shift energy levels, but they can also change the symmetry of the molecular orbitals (Kaasbjerg and Flensberg, 2011). Such symmetry breaking was invoked to explain the observations of unexpectedly many charging levels in the experiments by Kubatkin et al. (2003). The experiments were performed by low-temperature deposition of OPE5 molecules into a junction between metal leads, which led to weak metal-molecule coupling. The molecule in this configuration acted as a quantum dot, and 
by means of a back gate the charging state of the molecule could be varied. Up to eight charging levels could be reached in a gate voltage window of -4 to $+4 \mathrm{~V}$, which does not agree with homogeneous charging models for the molecule. The explanation comes from the distortion of the molecular levels by the image charges, by which local potential wells are formed at each end of the molecule near each of the two electrodes. This results in charging at the two ends of the molecules and keeps the charges at a distance from each other.

Together these experiments show that electrostatic effects have a major influence on molecular junction properties and lead to surprising effects. The effects are sensitive to the shape and geometry of the metal-molecule junction but, qualitatively, the effects are known and well understood.

\section{F. Current-voltage characteristics}

Diode characteristics in molecular junctions formed the start of the field (Aviram and Ratner, 1974) and are attractive because they represent the simplest two-terminal functional property of molecules. A general discussion of mechanisms for nonlinearities in $I-V$ traces was given in Sec. III.D.4. Here we consider the interpretation of experiments showing strongly nonlinear $I-V$ characteristics at high applied bias, i.e., far from equilibrium. This includes asymmetric $I-V$ characteristics for which the rectification ratio $R_{\mathrm{rr}}:=$ $|I(+V) / I(-V)|$ differs strongly from unity. It also includes nonmonotonic $I-V$ characteristics, where the current becomes smaller for increasing voltage above a certain threshold value. The latter is known as negative-differential resistance (NDR) and is also a sought-after property for use in devices such as oscillators and amplifiers. A recent review of diode characteristics in molecular junctions was given by Zhang et al. (2017), and the theory was presented in Sec. III.D.4.

Experiments started with work on Langmuir-Blodgett films (Geddes et al., 1990; Metzger et al., 2003), which demonstrated that the $D-\sigma-A$ type of molecules proposed by Aviram and Ratner indeed show asymmetric $I-V$ characteristics (here $D$ is a donor group, $A$ is an acceptor group, and $\sigma$ represents a coupling by $\sigma$ bonds). However, they also showed that the properties of such junctions can be richer than anticipated because the asymmetry sometimes had the opposite sign.

With the advent of single-molecule techniques it soon became apparent that asymmetry is a rather common feature in molecular $I-V$ characteristics, even for nominally symmetric molecular systems, an extreme example of which was presented in Sec. IV (Capozzi et al., 2015). Conversely, when a molecule has an asymmetric structure this property alone is not enough for producing a large rectification ratio.

Simple one-level models are helpful for obtaining a first interpretation for many of these observations. For the $I-V$ curves to be asymmetric the left-right mirror symmetry of the junction needs to be broken in at least one of many ways.

As illustrated in Fig. 24(a), the asymmetry may result from different barrier widths produced by asymmetric coupling of the molecule. In this case, the voltage drop is largely concentrated at the wide barrier. The schematics in Fig. 24(a) apply for molecules having molecular orbitals that are delocalized over the full length of the molecular backbone. The externally applied electrical potential is assumed to (a)

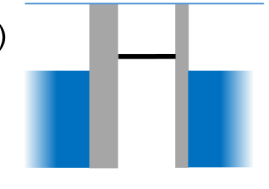

$\mathrm{V}=0$
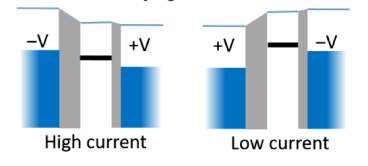

(b)
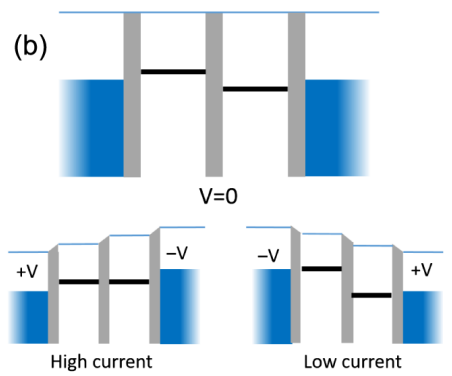

FIG. 24. Schematics of molecular levels leading to asymmetric and nonmonotonous $I-V$ characteristics. In these diagrams the vertical scale represents energy, while the horizontal axis is a space coordinate. Filled states in leads are indicated by the blue shaded range. The lines at the top show the local electrostatic potential (vacuum level). The gray bars show effective energy barriers. The asymmetry can be due to differences in coupling to the two leads, represented by different barrier widths (a). When the molecule has two sites separated by an internal barrier (b), the current becomes for coherent transport high when the two levels are shifted into resonance by the applied potential. Level alignment was not considered in the original proposal by Aviram and Ratner. In that proposal, the high current was taken to result from a cascade between the levels, involving relaxation by vibron excitation.

produce voltage drops only over the linker groups to the molecular backbone, here represented as tunneling barriers, which is not a valid assumption in general (see the forthcoming discussion).

The mechanism of Aviram and Ratner requires two levels and is described by a $D-\sigma-A$ type structure of the molecule. The mechanism proposed is based on incoherent hopping transfer between the $A$ and $D$ sites, which works only when the coupling between the molecule and the leads is weak. As a consequence, in this case the total current will be extremely small. Moreover, charging effects are likely to modify the outcome fundamentally.

When the coupling to the leads is stronger, coherent transport also produces asymmetric $I-V$ curves as a result of the shift of the relative positions of the two levels with respect to each other as a function of the applied voltage (Elbing et al., 2005); see Fig. 24(b). At higher bias, when the levels cross the same mechanism also produces NDR (Perrin et al., 2015, 2016).

Fusing $D$ and $A$ moieties together directly may be viewed as a molecular representation of a $p$ - $n$ junction in semiconductors (Ng, Lee, and $\mathrm{Yu}, 2002)$. However, the sign of the asymmetry of the $I-V$ curves does not generally agree with this picture. The mechanism of the observed diodelike characteristics may be produced by voltage-induced breaking of the delocalization of the wave function across the molecule; see Sec. III.D.4 and Zhang et al. (2017).

The widely observed asymmetries are often referred to as diode characteristics. However, an asymmetric $I-V$ does not imply that the system is useful as a diode. Large rectification ratios $R_{\mathrm{rr}}$ are difficult to achieve at the single-molecule level. Commercial diodes have $R_{\mathrm{rr}}$ of the order of $10^{5}-10^{8}$. The model calculations presented by Armstrong et al. (2007) and Garrigues et al. (2016) suggest that there is a maximum for $R_{\mathrm{rr}}$ 
for a molecular diode of about $10^{2}$ or $10^{3}$, respectively. However, the model assumptions underlying these estimates are not general enough to cover all possible molecular diode systems. Indeed, in recent experiments rectification ratios have been reported of up to $10^{3}$ (Capozzi et al., 2015; Perrin et al., 2016), up to $10^{4}$ (Ateşçi et al., 2018), and even $10^{5}$ (Chen et al., 2017). Explanations for the large rectification ratios invoke the role of two levels in the molecule or additional electrostatic effects.

The full problem of nonequilibrium junctions and the evaluation of the $I-V$ characteristics is quite involved because local charge may change in response to the flowing current, the nature and shape of the molecular orbitals is strongly affected by the field gradient, and the potential profile needs to be calculated self-consistently, setting apart further complications due to inelastic excitations. Few self-consistent calculations have been performed. However, such computations are of prime importance for the interpretation of systematic trends and for uncovering specific physical mechanisms (Ruben et al., 2008; Perrin et al., 2013). Stokbro et al. (2003) and Arnold, Weigend, and Evers (2007) showed how complicated the problem may become. These calculations were done for a symmetric molecule benzeneditiol, and similar computations for larger molecules are quite demanding. A self-consistent calculation for an asymmetric molecule, which incorporates a cobaltocene group, was done by Liu et al. (2006). The results show substantial asymmetry, but the rectification ratio remains modest, below 30 . More important is that the self-consistent calculations showed that the profile of the electrical potential agreed neither with the assumption that the potential drops over the connections of the molecule to the leads nor the assumption that it drops over the molecule itself. The actual potential profile computed shows a combination of the two, and the shape of the profile changed upon reversing the polarity. The conclusion should be that simplified models may be helpful as a guide but are unlikely to be highly accurate for describing properties of molecules far from equilibrium, as needed for the description of $I-V$ characteristics.

\section{G. Thermal and thermoelectrical properties}

A difference in temperature $\Delta T$ between the two leads connecting a molecule in a junction induces a current across the junction, which is known as the Seebeck effect. Under open-circuit (or high external impedance) conditions a potential difference builds up that counteracts this current. Upon reaching equilibrium the voltage induced by the temperature difference is given by the Seebeck coefficient $S$ (or the thermopower) of the junction $\Delta V=S \Delta T$, with (van Houten et al., 1992)

$$
S=-\left.\frac{\pi^{2} k_{B}^{2} T}{3|e|} \frac{\partial \ln [\mathcal{T}(E)]}{\partial E}\right|_{E=E_{F}}
$$

Here $\mathcal{T}(E)$ is the energy-dependent transmission and $T$ is the average temperature. A picture of noninteracting particles has been assumed.
Measurements of the thermopower provide additional information on the electronic properties of the junction (Ludoph and van Ruitenbeek, 1999). For single-molecule junctions, through Eq. (54) the sign of $S$ immediately translates into the nature of the nearest molecular orbital (HOMO or LUMO), ${ }^{23}$ and the combined knowledge of conductance and thermopower gives information on the distance of this level to the Fermi energy of the leads (Paulsson and Datta, 2003; Reddy et al., 2007; Malen et al., 2009). Three methods for measuring thermopower have been reported: by measuring the thermally induced voltage (Ludoph and van Ruitenbeek, 1999), by measuring the thermally induced current (Widawsky et al., 2013), and by taking $I-V$ curves with a temperature difference across the junction (RincónGarcía et al., 2016).

Reddy et al. (2007) reported the first single-molecule thermopower measurements on 1,4-benzenedithiol, 4,4'dibenzenedithiol and 4,4"-tribenzenedithiol and demonstrated that these form $p$-type molecular junctions, i.e., the Fermi energy lies close to the HOMO. Malen et al. (2009) studied three classes of molecules: phenylenediamines, phenylenedithiols, and alkanedithiols of various length. The phenylene molecular wires show a thermopower that grows linearly with length of the molecular backbone, while the alkanes show the opposite trend. Electrical transport through alkanedithiols connected between gold leads had previously been argued to be influenced by gateway states at the connection to the leads (Zeng et al., 2002; Zhou et al., 2008), but it was difficult to separate the contributions of in-gap states from those of HOMO or LUMO tunneling. By the additional information obtained through thermopower, Malen et al. (2009) demonstrated the importance of gateway states.

Generally, the position of the molecular levels, and therewith the transmission as a function of energy, is asymmetric with respect to the Fermi energy and, therefore, breaks electron-hole symmetry. While this leads to finite thermopower, the opposite effect can also be observed: imposing a current leads to asymmetric heating. The latter is more difficult to observe, and for this purpose Lee et al. (2013) developed an advanced nanoscale thermocouple integrated into a scanning tunneling probe. The probe measures the temperature of the tip apex contacting the molecule with a thermocouple mounted approximately $300 \mathrm{~nm}$ from the tip apex. Using this probe, they studied heat dissipation in a system formed by attaching single molecules of 1,4-benzenediisonitrile and 1,4-benzenediamine between Au electrodes. They showed that even for symmetric molecules the heat dissipation takes place asymmetrically across the junction and depends on the applied bias polarity and the sign of the Seebeck coefficient $S$. For positive $S$ the electrons dissipate more heat at the junction from which they originate, while for negative $S$ more heat is dissipated at the receiving end. These

\footnotetext{
${ }^{23}$ A note of caution: when quantum interference between the molecular orbitals plays a role near the Fermi level, this unambiguous interpretation may be lost; see Sec. V.D.
} 
techniques have been extended to allow measurements of the thermal conductance of atomic and molecular junctions (Cui, Jeong et al., 2017). For a comprehensive review on this topic, see Cui, Miao et al. (2017).

An interesting theoretical contribution with general validity for measurements of Seebeck coefficients was recently made by Rix and Hedegård (2019). It brought together a topic of Sec. IV, circulating currents, and the Seebeck measurement. Rix and Hedegård observed that the measurement of $S$ as the ratio $\Delta V / \Delta T$ at zero charge current implies that the divergence of the associated current density vanishes $\operatorname{div} \mathbf{j}(\mathbf{r})=0$, but not $\mathbf{j}(\mathbf{r})$ itself. Circulating currents are possible under these conditions and do, in general, emerge. The nearlyopen-circuit conditions of thermopower experiments suppress the regular current and permit detection of the effects of the circulating currents. Evidently, such experiments will be challenging.

\section{H. IETS and sign inversion}

Electronic transport through single molecules serves as an interesting playground for studying inelastic interactions of electrons with vibration modes (or "vibrons") in a single molecule. The inelastic signal offers a means of spectroscopy that aids in confirming the presence of the molecule under testing.

The strength of the electron-vibron coupling governs the nature of the electron transport. In the majority of the experiments reported in the literature, the electron transit time (i.e., the inverse level broadening) in the molecule is short compared to the time needed for the ions to respond. The interaction can then be described by perturbation theory at the level of the Born-Oppenheimer approximation. In the other limit, the transport is incoherent and leads to polaron formation on the molecule. Examples that classify into this limit are the experiments on long oligomers beyond the transition from coherent to incoherent transport, described in Sec. V.A, and the Franck-Condon blockade to be discussed later. Here we limit the discussion to weak electron-phonon coupling.

The resolution of the experimental signal relies on a sharply defined Fermi level in the metal electrodes, which implies that clear signals are found only at low temperatures. The first measurements demonstrating electron-vibron interactions on single molecules (Stipe, Rezaei, and Ho, 1998) were done for a single acetylene $\left(\mathrm{C}_{2} \mathrm{H}_{2}\right)$ molecule on a $\mathrm{Cu}(100)$ surface in a low-temperature UHV STM setup. A small but well-defined step upward toward an increased conductance was observed at a voltage equal to $\hbar \omega / e$, the energy of a vibrational excitation of the molecule. This measurement was done in tunneling mode, at an electron transmission $\mathcal{T} \ll 1$ and the increase in conductance at energy $\hbar \omega$ was attributed to the opening of an additional inelastic transport channel.

Measurements of vibration modes on a single molecule bound symmetrically between two leads were first done for a single $\mathrm{H}_{2}$ molecule and Pt leads (Smit et al., 2002; Djukic et al., 2005). In this case a step downward, i.e., a decrease in the molecular conductance, was recorded. ${ }^{24}$ The critical difference between the two experiments is the transmission $\mathcal{T}$, which is close to unity for the latter experiment. A similar decrease in conductance at transmission close to unity was also recorded for monoatomic chains of $\mathrm{Au}$ atoms (Agraï et al., 2002).

The crossover in sign of the inelastic signals has been investigated near equilibrium perturbatively in the electronphonon coupling energy $g$ using numerical (Paulsson et al., 2008) and analytical approaches (Egger and Gogolin, 2008; Entin-Wohlman, Imry, and Aharony, 2009). The latter directly employ the Holstein model, but the former approach also effectively reduces to this model when adopting the lowestorder expansion

$$
\begin{aligned}
\hat{H}= & \left(\varepsilon_{0}+g \hat{Q}\right) \hat{d}^{\dagger} \hat{d}+\hbar \omega_{0} \hat{a}^{\dagger} \hat{a}+\sum_{k}\left(V_{k} \hat{c}_{k}^{\dagger} \hat{d}+\text { H.c. }\right) \\
& +\sum_{k} \epsilon_{k} \hat{c}_{k}^{\dagger} \hat{c}_{k},
\end{aligned}
$$

where $\hat{Q}=\hat{a}^{\dagger}+\hat{a}$ is the displacement operator. It features a single fermionic level coupled to a bosonic vibration mode with frequency $\omega_{0}$; for a perspective on strong-coupling phenomena in this model, see Thoss and Evers (2018).

The structure of the general result for IETS corrections as it emerges from the Holstein model can be analyzed employing standard dimensional analysis. For simplicity, we consider the case of symmetric coupling $\Gamma=\Gamma_{\mathcal{L}}=\Gamma_{\mathcal{R}}$ and adopt the wideband limit, in which $\Gamma$ is independent of energy. Focusing on zero temperature, we have the parametric dependency

$$
d I / d V=e^{2} / h \mathfrak{f}\left(V_{b}, \varepsilon_{0}, \Gamma, \omega_{0}, g\right),
$$

with $\mathfrak{f}$ a dimensionless function of its arguments that includes elastic and inelastic scattering processes. We are interested in the corrections to the $d I / d V$ curve induced by the coupling $g$. The natural dimensionless small parameter will be $g / \Gamma$. In the limit $g \ll \Gamma$, the dwell time of the electron in the process of traversing the level $\hbar / \Gamma$ is too small for the action, which is related to the electron-vibron coupling, to become effective. The leading IETS signal will be determined by the expression of second order in this small parameter, ${ }^{25}$

\footnotetext{
${ }^{24}$ Kristensen et al. (2009) using first-principles calculations showed that a step-up in conductance for such $\mathrm{Pt}-\mathrm{H}_{2}-\mathrm{Pt}$ systems should also be possible. This was attributed to the opening of an otherwise closed $d$ channel, along with the usual $s$ channel, when exciting a transverse hindered rotation mode for the $\mathrm{H}_{2}$ molecule. Recently, such a step-up has also been found in experiments (Tewari, Sabater, and van Ruitenbeek, 2019). As pointed out there, these steps in conductance are small and it is extremely difficult to distinguish them from the structures that could appear due to elastic scattering of electronic waves through defects in the Pt leads.

${ }^{25}$ The first-order term in $g$ is proportional to the oscillator's displacement out of its equilibrium position. It is higher order in $V_{b}$ and can be ignored when the vibrational relaxation is fast enough.
} 


$$
\left.\frac{d I^{\mathrm{IETS}}}{d V}\right|_{e V_{b} \gtrsim \hbar \omega_{0}} \approx \frac{e^{2}}{h} \frac{g^{2}}{\Gamma^{2}} \mathfrak{f}^{(2)}\left(\varepsilon_{0} / \Gamma, \omega_{0} / \Gamma\right) .
$$

It was already written for the case $e V_{b} \gtrsim \hbar \omega_{0}$ since it is at these voltages where the sign of the IETS correction manifests itself. We accounted for $\mathfrak{f}^{(2)}$ being dimensionless, so its three arguments combine into two dimensionless ratios.

In general, $\mathfrak{f}^{(2)}$ is a complicated function of its parameters (Egger and Gogolin, 2008; Entin-Wohlman, Imry, and Aharony, 2009). It takes a transparent form in the limit of a soft (extremely slow) vibration, i.e., $\hbar \omega_{0} / \Gamma \rightarrow 0$ in Eq. (56),

$$
\left.\lim _{\hbar \omega_{0} / \Gamma \rightarrow 0} \frac{d I^{\mathrm{IETS}}}{d V}\right|_{e V_{b} \gtrsim \hbar \omega_{0}} \approx \frac{e^{2}}{h} \frac{g^{2}}{\Gamma^{2}} \mathfrak{f}^{(2)}\left(\varepsilon_{0} / \Gamma, 0\right) .
$$

Intuitively, Eq. (57) describes the jump of the conductance when the bias voltage crosses the threshold energy $\hbar \omega_{0}$ under the assumption that the phonon frequency is still small compared to the level broadening.

After this step the model has in fact been simplified to such an extent that the sign of $\varepsilon_{0}$ no longer matters, because all reference scales except zero energy have dropped out. Consequently, the rhs of Eq. (57) depends only on $\left(\varepsilon_{0} / \Gamma\right)^{2}$ and therefore can also be considered a function of the zerobias transmission $\mathcal{T}$, which is an invertible function of the same argument. Summarizing, within the model assumptions the IETS signal follows a "universal" function, i.e., independent of microscopic model parameters, with the transmission $\mathcal{T}$ as the only remaining variable describing the molecular bridge at hand

$$
\left.\lim _{\hbar \omega_{0} / \Gamma \rightarrow 0} \frac{d I^{\mathrm{IETS}}}{d V}\right|_{e V_{b} \gtrsim \omega_{0}} \approx \frac{e^{2}}{h} \frac{g^{2}}{\Gamma^{2}} f(\mathcal{T}) .
$$

For the case $\Gamma_{\mathcal{L}} / \Gamma_{\mathcal{R}}=: \alpha$ with $\alpha \neq 1$, a more general result can be obtained along similar lines; we are then left with a twoparameter dependency $f(\mathcal{T}, \alpha)$.

The function $f(\mathcal{T}, \alpha)$ was calculated and discussed by Paulsson et al. (2008). At high transmission $\mathcal{T} \simeq 1$, the forward-scattering electronic states are nearly fully occupied, implying that electrons that undergo inelastic scattering by excitation of a vibron can find unoccupied states only by scattering backward. In the other limit $\mathcal{T} \ll 1$, nearly all incoming states are scattered back elastically such that after an inelastic scattering event with a vibron the electron finds empty states only in the forward-scattering direction. Therefore, we expect a tendency that inelastic scattering leads to a decrease of the conductance at high transmission $f<0$, and to an increase in conductance at low transmission $f>0$. The crossover occurs on a line in the $(\alpha, \mathcal{T})$ plane where $f(\alpha, \mathcal{T})=0$. The line was calculated by Paulsson et al. (2008); see also Kim and Son (2013). For soft vibrations and symmetric coupling, i.e., $\alpha=1$, the crossover takes place at $\mathcal{T}=0.5$.

We emphasize that the model analysis is based on the wideband limit and on assuming soft vibrations with an extremely fast relaxation mechanism. The more general problem also includes the backaction of the nonequilibrium vibron occupation, which results in a shift of the crossover point to higher transmission and therefore is considerably more complicated. A first attempt at solving the problem was made by Urban, Avriller, and Levy Yeyati (2010), who, however, neglected backaction related frequency renormalizations $^{26}$ (Kaasbjerg, Novotný, and Nitzan, 2013). More systematic treatments have been presented in subsequent work that have, in particular, also included this effect (Novotný, Haupt, and Belzig, 2011; Utsumi et al., 2013; Ueda et al., 2017); for a numerical solution of the Holstein model including an analysis of IETS, see Schinabeck et al. (2016).

Experimentally, a crossover between a step-up and a stepdown at the bias voltage corresponding to the excitation of a molecular vibration was observed for single-molecule $\mathrm{H}_{2} \mathrm{O}$ junctions between Pt leads (Tal et al., 2008), and for benzenedithiol between $\mathrm{Au}$ leads (Kim, Pietsch et al., 2011). The crossover point in the former experiment was found near $\mathcal{T} \approx 0.65$, but from shot noise measurements on the same junctions it was found that a second conduction channel contributes to the conductance. The dominant conductance channel has a transmission near 0.5 at the crossover point. The experiments by Kim, Pietsch et al. included an analysis of the asymmetry of the coupling to the two leads $\alpha=\Gamma_{\mathcal{R}} / \Gamma_{\mathcal{L}}$ that could be obtained from the shape of the current-voltage characteristics. From a plot of the inelastic signal intensity normalized to the conductance on the junction, a clear crossover was obtained near $\mathcal{T} \approx 0.5$. In a follow-up piece of research, Karimi et al. (2016) demonstrated, using shot noise measurements, that the electron transport in BDT molecules is indeed due to a single conductance channel for $\mathcal{T}$ ranging from 0 to 0.6 .

As emphasized by Avriller and Levy Yeyati (2009), Haupt, Novotný, and Belzig (2009), and Schmidt and Komnik (2009), the conductance is just the first moment of the distribution of electron transfer probabilities. Inelastic signals are expected to show up in all moments of the distribution of electron transfer, and, in particular, in the second moment, which is known as shot noise. For symmetric junctions, at transmission $\mathcal{T} \simeq 1$ inelastic scattering is expected to produce increased noise signals. A crossover to a negative contribution takes place near $\mathcal{T} \approx 0.85$, and another crossover back to a positive noise contribution takes place at $\mathcal{T} \approx 0.15$, again under the assumption of a large width of the electronic level. This inelastic noise signal was observed for short $\mathrm{Au}$ atomic chains (Kumar et al., 2012), and a crossover was seen from positive to negative inelastic noise contributions. However, the crossover point was found near $\mathcal{T} \approx 0.95$, higher than predicted for the simple model systems. The rather large value for the turnaround point was addressed in a subsequent theoretical study by Avriller and Frederiksen (2012), who adopted the approach of Haupt, Novotný, and Belzig $(2009,2010)$ for an $a b$ initio treatment. The study did not observe any sign change at all, so the discrepancy between experiment and different theories appears to persist. More recently, by a hierarchical quantum master equation approach, Schinabeck and Thoss (2019) obtained a shift in the transition between positive and

\footnotetext{
${ }^{26}$ We acknowledge Tomáš Novotný for bringing these developments to our attention.
} 
negative inelastic noise contributions that they attributed to the nonequilibrium occupation of vibrational modes. Unfortunately, the shift makes the discrepancy with experiment larger, not smaller.

IETS is not governed by strict selection rules such as those that apply to other forms of spectroscopy, including Raman scattering and IR absorption and emission. Nevertheless, symmetries of the molecular orbitals and symmetries of the vibration modes involved in the electron scattering lead to approximate selection rules that are known as propensity rules (Troisi and Ratner, 2006; Gagliardi et al., 2007). For example, Gagliardi et al. (2007) showed that for all molecules bound to Au through a sulfur atom the IETS spectrum is dominated by the totally symmetric vibration modes. The amplitudes, on the other hand, are difficult to predict because they are extremely sensitive to interference between various contributions to the inelastic signal. Another important difference from the other forms of spectroscopy is the intrinsic limitation in resolution due to the strong coupling of the vibration modes to those of the metal leads and due to the hybridization of the electronic levels of the molecule with the bulk states in the leads. Only in limiting cases, when the molecule is weakly coupled to both leads, does a more quantitative comparison between theory and experiment become possible. This was demonstrated by Krane et al. (2018) in low-temperature STM experiments, where a monolayer of $\mathrm{MoS}_{2}$ served to decouple the molecules from the substrate.

Lykkebo et al. (2013) predicted that overtones, i.e., multiples of the fundamental vibration frequencies, may in some cases dominate the IETS spectrum. To our knowledge observation of overtones has not been reported for regular IETS spectra, but we see in Sec. V.J that overtones dominate the spectra under conditions of the Franck-Condon blockade.

\section{Coulomb blockade and the Kondo effect}

The theory of Coulomb blockade and the Kondo effect was reviewed in Sec. IV. Here we show how molecular junctions allow testing of long-standing predictions and offer means of preparing new electron-correlated phases. While our review focuses on molecules contacted to leads, in this section we mention a few STM experiments that operated in the tunneling regime. The development of our understanding of quantum transport through open-shell molecules enjoyed cross talk of both experimental approaches (Scott and Natelson, 2010).

\section{The single-impurity Anderson model in single-molecule junctions}

While the SIAM applies to various physical systems (Kouwenhoven and Glazman, 2001; Scott and Natelson, 2010), molecular junctions occupy a prominent role because they allow for quantitative testing of theoretical predictions in a wide parameter window.

Zhang et al. (2013) studied the $\mathrm{C}_{28} \mathrm{H}_{25} \mathrm{O}_{2} \mathrm{~N}_{4}$ neutral radical adsorbed on $\mathrm{Au}(111)$. In the differential conductance, a zerobias resonance (ZBR) appears. The latter is associated with the spin of the unpaired electron of the radical. The strong temperature dependence of the ZBR, without saturation at low $T$, points to the weak-coupling Kondo regime $T \gg T_{K}$, where spin-flip scattering can be described by a perturbation theory in the Kondo exchange coupling $J$; see Eq. (31) (Kondo, 1964; Appelbaum, 1966). ${ }^{27}$ To third order in $J$ the conductance at zero magnetic field can be expressed by

$$
G(e V, T)=G_{\mathrm{bg}}+A f\left(e V / k_{B} T\right)
$$

where $A$ is proportional to $J^{3}$ and the function $f$ is independent of microscopic parameters. The background contribution $G_{\mathrm{bg}}$ is treated as a constant. Equation (59) can be easily generalized to finite magnetic fields, where the only additional parameter is the gyromagnetic constant $g$, which determines the Zeeman energy and consequently the splitting of the ZBR into two symmetrically positioned steps in the differential conductance (Appelbaum, 1967). Zhang et al. test the accuracy of this theory by treating the temperature $T$ and $g$ as fitting parameters. The $T$ obtained from the fits agrees with the experimental temperature to within an accuracy of $10 \%$ (unless $T<6 \mathrm{~K}$, where the perturbation theory is not expected to be successful). The value for $g=1.93 \pm 0.02$ is slightly reduced compared to the gas phase, reflecting the screening of the $g$ factor by the electron gas in the $\mathrm{Au}$ substrate.

Extending quantitative tests of the theory for single molecules suspended between two leads down to temperatures below $T_{K}$ ("strong-coupling" Kondo regime) poses a significant experimental challenge, namely, the stability of the system across temperature differences spanning several orders of magnitude. This difficulty can be bypassed in molecular junctions where, instead of varying the temperature, stretching of the molecular junction drives changes of $T_{K}$ by varying the microscopic parameters. Žonda et al. (2018) took this approach to investigate the ZBR in perylenetertracarboxylic dianhydride (PTCDA) suspended between Ag contacts. The theoretical apparatus involves conductance calculations based on the solution of the SIAM with the numerical renormalization group (Wilson, 1975; Bulla, Costi, and Pruschke, 2008). The fitting procedure involved only two parameters, the couplings to the two leads $\Gamma_{\mathcal{L}}$ and $\Gamma_{\mathcal{R}}$, but successfully captured the conductance spectra in the bias window $\pm 15 \mathrm{mV}$ in both strong-coupling and weak-coupling regimes.

\section{Two-impurity Anderson model}

Following the theoretical overview in Sec. IV.A.3, we present a discussion of molecules with two relevant orbitals in quarter and half fillings.

Quarter filling: The SU(4) Kondo effect.-Good realizations of the SU(4) Kondo effect on a single entity were given in carbon nanotubes (Jarillo-Herrero et al., 2005; Makarovski et al., 2007; Schmid et al., 2015). In this case, the orbital degeneracy required for an SU(4) Kondo effect arises from the valley degree of freedom. However, the contacts and impurities induce mixing of the two orbitals, and therefore disturbances of the SU(4) symmetry are significant. In molecular quantum dots, such perturbations can be avoided

\footnotetext{
${ }^{27}$ The perturbation approach breaks down when $T \approx T_{K}$.
} 
by imposing point symmetry of the compound system (molecule and contacts). Consider the case of transition-metal phthalocyanines $(\mathrm{Pc})$ adsorbed on a crystalline metallic surface. The gas-phase Pc is symmetric around a fourfold axis, which gives rise to doubly degenerate levels. The fourfold axis can be preserved when the molecule is placed on a cubic lattice. This is the case for nickel and copper phthalocyanines (NiPc and $\mathrm{CuPc}$ ) adsorbed on $\mathrm{Ag}(100)$ (Korytár and Lorente, 2011; Mugarza et al., 2012). The degenerate ligand states $\left(e_{g}\right)$ contain a single electron in the Coulomb-blockade regime. The SU(4) character manifests itself in STM images of the Kondo resonance, which is uniformly detected over the ligands of the Pc.

An SU(4) Kondo effect was also found for FePc adsorbed on two distinct sites on $\mathrm{Au}(111)$ (Minamitani et al., 2012). The gas-phase FePc has a doubly degenerate level, residing mainly on the $d$ shell. The molecules are adsorbed on Au(111) on one of two sites, either the "on-top" or the "bridge" site. The analysis of STM spectra with and without magnetic field suggests that at the on-top adsorption site the Kondo effect is of the SU(4) type, and at the bridge site it turns into an SU(2) type. This observation correlates well with the strongly reduced symmetry of the bridge site. The molecular SU(4) Kondo system allows the molecules to be assembled into a 2D array, as demonstrated by the same group (Tsukahara et al., 2011). Such an SU(4) Kondo lattice offers means to study the competition between orbital and spin ordering (Lobos, Romero, and Aligia, 2014; Fernández, Aligia, and Lobos, 2015).

These results suggest that the delicate conditions required for the SU(4) Kondo effect may be achieved in molecules more easily than in other systems. The orbital mixing, e.g., can vanish in carbon nanotubes due to symmetry reasons. Additionally, we remark that the SU(4) Kondo effect can be disturbed by certain interaction matrix elements, i.e., the terms in Eq. (36). The latter can have a lower symmetry in molecules than in carbon nanotubes.

Molecules with two open shells.-Molecules with two open shells can be understood as having two unpaired spins. This allows for the realization of several important regimes of the TIAM at half filling. These regimes are controlled by the sign and magnitude of the exchange coupling; see Sec. IV.A.3. When the two respective orbitals belong to the same $d$ shell, the exchange is ferromagnetic (Coulomb exchange, Hund's rule) and strong $(\approx 1 \mathrm{eV})$, effectively locking both spins into a spin-1 moment. When the overlap between the two orbitals is small, Coulomb exchange is negligible and other mechanisms take a leading role (superexchange, RKKY exchange). These mechanisms are sensitive to the details of the system and the environment, allowing for in situ tuning, as shown later.

Underscreened Kondo effect.-The signatures of an underscreened Kondo effect were observed for the first time in a single entity, a $\mathrm{C}_{60}$ molecule in a gold nanogap, by Roch et al. (2009). The key in the identification of the underlying spin model was the comparison of the temperature dependence of the conductance with the universal dependencies obtained from the numerical renormalization group (NRG) calculations $G(T)=G_{0} f_{S}\left(T / T_{K}\right)$ for a given impurity spin $S$. A second important hallmark of underscreened Kondo physics is the splitting of the Kondo peak in a magnetic field. Consistent with expectations for the underscreened Kondo effect, Roch et al. observed that the field required to split the peak is small. The interplay of the Kondo correlations of a spin-1 system and magnetic anisotropy was studied by Parks et al. (2010) in a Co complex. By mechanically distorting the ligand field around the Co, the anisotropy energy can be manipulated, providing means to switch on and off the underscreened correlations without a magnetic field.

Singlet-triplet transitions.- - Roch et al. (2008) reported the observation of a quantum phase transition in an electromigrated $\mathrm{C}_{60}$ junction. As Roch et al. argued, the transport measurements can be interpreted by the two-impurity Kondo model with only a single screening channel at the accessible experimental temperatures. An important manifestation of the quantum critical point is the appearance of a two-stage Kondo screening process on the singlet side of the transition. In the transport measurements, the second-stage Kondo effect is detected by the formation of a dip in the middle of a broad first-stage Kondo peak.

An interesting option to experimentally observe the singlettriplet transition is to bring two open-shell molecules into contact. Esat et al. (2016) studied a PTCDA-Au complex adsorbed on the surface of gold. The LUMO of the complex is a delocalized $\pi$ orbital which captures a single electron upon adsorption. When two PTCDA-Au complexes are brought close to each other both LUMO's overlap. As Esat et al. argued, the direct exchange interaction is negligible, and the physics is dictated by the charging energy of the LUMOs along with the intermolecular hybridization. The pairs of PTCDA-Au complexes can be found in various on-surface orientations, as shown by the STM images. The zero-bias features in the tunneling conductance are either a single Kondo peak or a split resonance. The occurrence of two kinds of zero-bias features is rationalized by NRG calculations on a TIAM, showing that they correspond to underscreened triplet and singlet phases of the two-molecule systems. Both phases are separated by a quantum phase transition, driven by changing the orbital hybridization $t$. Esat et al. employed $a b$ initio calculations of pairs of gas-phase complexes in the orientations observed experimentally. The $t$ was estimated from the splitting of the LUMO energies. The $t$ calculated in this way correlates well with the observation of the split peak.

A prototypical two-spin molecule (a complex with two $\mathrm{Ni}^{2+}$ centers) was studied by Zhang et al. (2015) by combining STM measurements on $\mathrm{Cu}(100)$ and first-principles calculations. They argued that the exchange interaction between the two spins is much smaller than the Kondo temperature, so both spins are independently Kondo screened. The regime of independent Kondo screening becomes interesting when larger spin arrays are arranged on the surfaces; see, e.g., DiLullo et al. (2012). Such a molecular Kondo lattice may exhibit a strongly renormalized Fermi-liquid phase known as the heavy-fermion phase; see, e.g., Coleman (2015).

\section{The Kondo effect as evidence for an open-shell structure}

We pointed out in Sec. IV.A.2 that above the Kondo temperature it can be tricky to differentiate between openshell and closed-shell molecules solely based on transport 


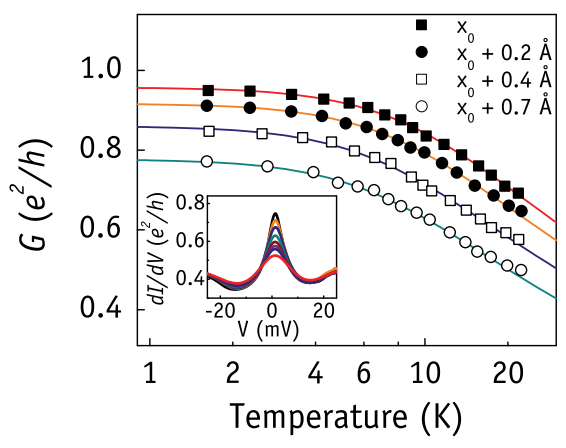

FIG. 25. Temperature dependence of the linear conductance of $\mathrm{C}_{60}$ in a gold junction for different electrode separations. Solid lines are fits to $G(T)=G_{0}\left[1+\left(T / T_{K}\right)^{2}\left(2^{1 / s}-1\right)\right]^{-s}+G_{b}$, where $s=0.22$ and $G_{b}, G_{0}, T_{K}$ are parameters. The widely used formula is a fit to the theoretical $G(T)$ from a numerical renormalization-group calculation (Costi, Hewson, and Zlatić, 1994; Goldhaber-Gordon et al., 1998). The extracted $T_{K}$ are (from the top trace to the bottom) $60.3 \pm 2.4,55.5 \pm 0.9$, $45.6 \pm 1.9$, and $38.1 \pm 1.2 \mathrm{~K}$. (Inset) $d I / d V$ traces at $x_{0}+0.7 \AA$ A. From Parks et al., 2007.

spectroscopy. The reason is that in molecular junctions the level spacing (e.g., the HOMO-LUMO gap) is typically comparable to the charging energy. The appearance of a Kondo resonance pinned at zero bias provides a direct proof of an open-shell nature of the contacted molecule, as illustrated in Fig. 25. It is not surprising that the Kondo resonance (and its sensitivity to microscopic details) allows one to gain qualitative insights into the charge transfer and level alignment of a molecule in contact with leads. We review a few examples where such an understanding was achieved. In these works, the interpretation of the experimental results was often backed up by $a b$ initio DFT calculations. Although state-of-the-art DFT incorrectly treats the Coulomb-blockade regime, it can provide qualitative insights into the charge state and the character of the relevant frontier orbitals of the contacted molecule.

The first series of examples (Parks et al., 2007; Temirov et al., 2008; Mugarza et al., 2011) comprises molecules that have a closed-shell configuration when neutral, but a Kondo resonance appears when the molecule is contacted. Temirov et al. (2008) studied PTCDA adsorbed on Ag. By contacting the molecule with a STM tip and gradually peeling off the molecule from the substrate, Temirov et al. recorded the $d I / d V$ spectrum and observed the emergence of a zero-bias resonance ZBR on top of a weakly varying background. The resonance width was approximately $15 \mathrm{meV}$. Standard tests of the Kondo effect are the magnetic field splitting and the temperature dependence; however, due to the rather large width of the ZBR such tests were not accessible. The identification of the ZBR with the Kondo resonance thus relied on the observed pinning to the position at zero bias and on comparison with the model and $a b$ initio calculations (Greuling et al., 2011). The findings can be rationalized by realizing that the peeling represents an inverse adsorption process: adsorbed PTCDA accommodates two electrons in the LUMO and a gradual decoupling should lead to redox transitions. A similar redox transition leading to the appearance of the Kondo effect was proposed by Karan et al. (2018) to explain the $d I / d V$ changes in an iron porphyrin-Au junction.

The intricacies of the level alignment further deepen when a molecule with two relevant orbitals is considered. Requist et al. (2014) studied a NO molecule adsorbed on Au(111). The Kondo resonance observed in STM was proposed to arise from a two-orbital system at quarter filling. DFT calculations offered the following scenario: the electron is distributed in both even and odd states $\left(2 \pi_{e}^{*}, 2 \pi_{\mathrm{o}}^{*}\right)$, but $2 \pi_{e}^{*}$ carries most of the electronic occupation. When the DFT results were contrasted with a NRG calculation, correlation effects invert the order of occupancies so that the odd $2 \pi_{o}^{*}$ orbital wins. We remark that in the case of NO adsorbents there is no symmetry protection for the even and odd states, in contrast to the SU(4) limit. New computational approaches are being developed that aim at improved $a b$ initio descriptions of strongly correlated phenomena in molecular junctions and adsorbents (Jacob, 2015; Droghetti and Rungger, 2017).

Molecular junctions offer new means of manipulating and controlling the microscopics that enters the Kondo physics. For example, Kondo resonances were switched on or off by changing the length of anchor groups (Park et al., 2002) using current-induced dehydrogenation (Zhao et al., 2005) or mechanical stretching (Rakhmilevitch et al., 2014). Pulses of electric current were used by Miyamachi et al. (2012) to switch a molecule from a spinful $(S=2)$ to a spinless $(S=0)$ state on the spin-crossover compound $\mathrm{Fe}(1,10 \text {-phenanthroline })_{2}\left[(\mathrm{NCS})_{2}\right]$ on $\mathrm{CuN} / \mathrm{Cu}(100)$. One of the keys to identifying the switching was the presence and absence of a Kondo peak in the respective spin states.

We recall the exponential sensitivity of the Kondo temperature on the microscopic parameters [Eq. (26)], which can be exploited as a sensitive probe of subtle interactions. Jacobson et al. (2015) studied $\mathrm{CoH}$ and $\mathrm{CoH}_{2}$ complexes adsorbed on a spatially corrugated surface [a hexagonal boron nitride monolayer on $\mathrm{Rh}(111)$ ] and systematically observed the Kondo ZBR only on adsorption sites of a specific type.

A robust ZBR indicates the conservation of localized radical spin-half character of a molecule. Such an observation was made by Frisenda et al. (2015) upon attaching a polychlorotriphenylmethyl radical to a pair of $\mathrm{Au}$ leads and stretching the junction in a MCBJ setup by $0.5 \mathrm{~nm}$. The ZBR retained its width $(\approx 5 \mathrm{mV})$, although the background conductance dropped 10 times.

An important feature of the differential conductance spectrum of open-shell molecules is the appearance of thresholds at finite bias, which are due to inelastic spin excitations (Hirjibehedin et al., 2007; Osorio et al., 2010; Fock et al., 2012; Gaudenzi et al., 2017). The finite-bias thresholds can be analyzed in external fields, supplied by the gate voltage and a magnetic field, which helps one determine the nature of the low-energy excitations of the open-shell system. Unlike vibrational signals in IETS, the IETS features due to spin excitations are characterized by selection rules due to spin conservation (to the extent that the spin-orbit interaction is weak). A remarkable consequence of the latter can be a suppression of zero-bias conductance, termed spin blockade (Romeike et al., 2007; de Bruijckere et al., 2019). Orbital 
(a)

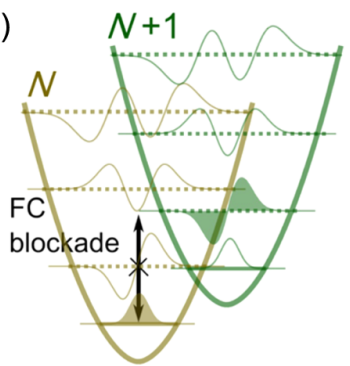

(b)

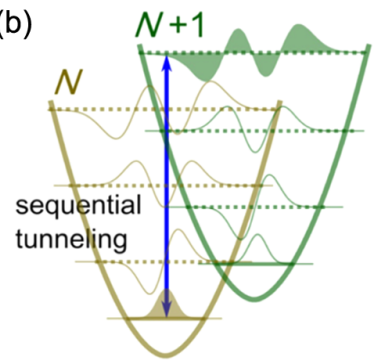

FIG. 26. Illustration of the Franck-Condon blockade in a molecular junction assuming a parabolic potential surface with eigenenergies $n \hbar \omega_{0}$ and vibronic (harmonic oscillator) wave functions. (a) At low excitation levels transport is suppressed because the oscillator overlap between $|N, 0\rangle$ and $|N+1,1\rangle$ is small. (b) At higher bias, higher vibrational levels $|N+1, n\rangle$, the overlap with $|N, 0\rangle$ is enhanced and current starts to flow. Adapted from Lau et al., 2016.

degrees of freedom lead to similar steps in the differential conductance (Kügel et al., 2018).

Fundamental questions related to electronic correlations were studied using single open-shell molecules coupled to ferromagnetic (Pasupathy et al., 2004; Fu, Xue, and Wiesendanger, 2012) and superconducting leads (Franke, Schulze, and Pascual, 2011; Hatter et al., 2015). However, a thorough discussion of these works is beyond the scope of this review.

\section{J. Franck-Condon blockade}

We consider a molecule, weakly coupled to the electrodes, with $N$ electrons in its vibrational ground state $|N, 0\rangle$. Furthermore, let the molecule be tuned near a chargedegeneracy point so that $|N, 0\rangle$ and $|N+1,0\rangle$ are nearly degenerate. If the electron-vibron coupling is strong, i.e., the reorganization energy is large, then the overlap $\langle N+1,0 \mid N, 0\rangle$ is small. Consequently, the low-bias current is suppressed even though charge fluctuations are not strongly hindered by Coulomb repulsion. At larger bias it is possible to excite the vibrational states $|N+1, n\rangle$. According to FranckCondon theory, the transition probabilities are proportional to the overlap squared $|\langle N, 0 \mid N+1, n\rangle|^{2}$ with

$$
|\langle N, 0 \mid N+1, n\rangle|^{2} \propto e^{-\lambda} \lambda^{n} / n !
$$

where $\lambda$ denotes the dimensionless electron-vibron coupling. For strong coupling $(\lambda \gg 1)$, the matrix element is exponentially small at $n=0$ but reaches a maximum for finite $n=n_{\max }$. Thus, transport is recovered at finite bias when the electrons have excess energy $e V$ large enough for exciting $\sim n_{\max }$ vibrons; see Fig. 26. The unique properties of electronic transport in the Franck-Condon blockade (FCB) regime have been analyzed theoretically by Kaat and Flensberg (2005), Koch and von Oppen (2005), Wegewijs and Nowack (2005), and Leijnse and Wegewijs (2008), among others. Note that the FCB derives from the suppression of overlap-matrix elements and hence cannot be lifted by tuning the gate voltage (see Fig. 27), in stark contrast to the Coulomb blockade. This behavior has been taken as a hallmark in the

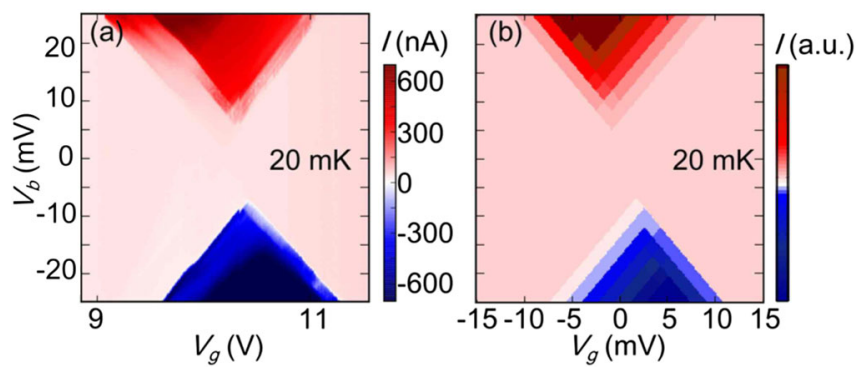

FIG. 27. (a) Current stability diagram of a functionalized $\mathrm{C}_{60}$ bound to graphene nanoelectrodes at $20 \mathrm{mK}$. (b) Simulated current stability diagram with $\lambda=3, \hbar \omega_{0}=1.7 \mathrm{meV}$. The current is suppressed for low bias voltage $\left|V_{b}\right| \lesssim 6 \mathrm{mV}$ because of the Franck-Condon blockade. The blockade cannot be lifted by changing the gate voltage $V_{g}$. For higher bias, the current is restored; its detailed $V_{g, b}$ dependence bears a strong imprint of vibronic excitations. From Lau et al., 2016.

experimental discovery of the FCB. In the context of nanoscale devices, the FCB was first observed in carbon nanotubes (Leturcq et al., 2009), where the blockade survives over several charge states $N$. A molecular FCB was first observed by Burzurí et al. (2014) in an $\mathrm{Fe}_{4}$ complex.

The FCB has some remarkable consequences for the charge-carrier dynamics (Koch, von Oppen, and Andreev, 2006). Above a threshold bias of a few times $\hbar \omega_{0}$ the electron current is accompanied by excitations and deexcitations of vibrons. The latter have their fingerprint in unusual temporal fluctuations of the current, i.e., in the current noise ("avalanche transport"). For an extended analysis of noise and avalanches, see Schinabeck et al. (2014).

The bistable behavior of the current in the FCB was observed in an experiment by Lau et al. (2016) for a functionalized $\mathrm{C}_{60}$ molecule coupled to graphene electrodes.The waiting times of the on and off phases were of the order of $10^{-1} \mathrm{~s}$, which is extremely long for molecular vibration processes. The FCB with avalanche transport was also manifested by giant enhancement of noise above the regular shot noise level $\left(10^{2}-10^{4}\right)$ in accord with the theoretical prediction (Koch and von Oppen, 2005).

\section{CASE STUDIES OF QUANTITATIVE COMPARISON}

Having reviewed some of the most impressive phenomena observed in single-molecule junctions, for which we have a high level of qualitative understanding, we now turn to a discussion of the quantitative level of agreement between computation and experiment. In many experiments, the conductance is the sole quantity reported, and consequently this is also what computations often focus on. It is useful to distinguish three conductance regimes, which leads us to organizing this section into three parts.

\section{A. High zero-bias conductance}

At high zero-bias conductance with quantum conductance channels having transmission probabilities near unity, the experiments give access to many parameters other than just the conductance. The systems we consider here are small 
molecules, with strong hybridization of the molecular levels with the metallic states in the leads. As a consequence, the level broadening is much larger than any other energy scale in the problem, which makes the computations less sensitive to the unknown details of the metal-electrode arrangement.

A prime example is a hydrogen molecule $\mathrm{H}_{2}$ coupled to $\mathrm{Pt}$ leads at either side (Smit et al., 2002). Clean Pt leads can be obtained by means of the MCBJ technique at cryogenic vacuum conditions, and the molecules can be introduced into the gap by deposition from the vapor phase. The conductance histogram after deposition of $\mathrm{H}_{2}$ shows many changes, indicating that many types of conducting bridges involving $\mathrm{Pt}-\mathrm{H}$ bonds are being formed, but a prominent peak near a conductance of $1 G_{0}$ is the most conspicuous feature. The experiments mainly focus on analyzing the structure of the bridge associated with this histogram peak.

The proposed configuration of the molecule in the contact is shown in Fig. 28. From a chemistry point of view, a more common arrangement of hydrogen between $\mathrm{Pt}$ atoms, with lower total energy, is one with the two hydrogen atoms aligned orthogonally to the junction axis, with one atom on either side. The latter configuration was proposed by García et al. (2004) as being associated with the conductance peak at $1 G_{0}$ in the histograms. In contrast, from their DFT-based computations they found a conductance of only $0.2 G_{0}$ for the configuration illustrated in Fig. 28. However, at least three independent calculations for the latter configuration produce a conductance extremely close to $1 G_{0}$ (Smit et al., 2002; Djukic et al., 2005; García-Suárez et al., 2005; Thygesen and Jacobsen, 2005a). By virtue of the many parameters that can be obtained from the experiments, including the vibration modes and their shift with isotope substitution, the contact stretching dependence of the vibration modes (Djukic et al., 2005), and the number of conductance channels derived from shot noise measurements (Djukic and van Ruitenbeek, 2006), the discrepancy between the calculations can be decided definitively in favor of the the latter group of computational results. Although the critical difference between these computations has not been identified, the example discussed here demonstrates the importance of benchmarking the computations against well-characterized experimental results. Barring the result by García et al. (2004), the quantitative agreement on the conductance between experiment and computations in this regime of transmission is near $10 \%$.

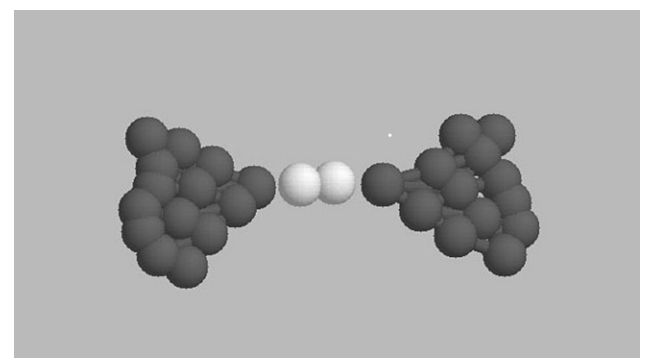

FIG. 28. Proposed Pt- $\mathrm{H}_{2}-\mathrm{Pt}$ molecular configuration. The dark spheres represent $\mathrm{Pt}$ atoms of the metallic leads, for which the detailed arrangement is not known. The white spheres represent the $\mathrm{H}$ atoms that are aligned with the axis of the junction.
At first sight, the high electron transmission of a closedshell molecule such as $\mathrm{H}_{2}$ is counterintuitive. An instructive discussion of the principles involved in arriving at this high conductance was given by Cuevas et al. (2003). Although the HOMO-LUMO gap for the $\mathrm{H}_{2}$ molecule is large, the $\mathrm{H}_{2}$ molecular orbitals hybridize strongly with the $d$ orbitals of Pt, which have a large density of states, resulting in a high electron transmission. The other key experimental observation, namely, that the conductance is carried by a just a single channel, is rooted in the axial symmetry of the molecular bridge illustrated in Fig. 28.

The approach of exploiting the chemical affinity of clean metal tips for direct binding to molecules, without the need for anchoring groups, has been extended to organic molecular systems, notably benzene (Kiguchi et al., 2008) and the related oligoacenes (Yelin et al., 2016). The more elaborate molecular orbital structure of these aromatic molecules permits multiple conductance channels for a single molecule. With a total conductance for a Pt-benzene-Pt junction in the range from $0.1 G_{0}$ to $1.3 G_{0}$. Analysis of shot noise shows that up to three channels participate in the electron transport. This agrees well with the accompanying DFT calculations, which offer the interpretation that the number of carbon atom bonds to each of the Pt tip atoms reduces from 3 to 1 in the process of gradual breaking of the junction (Kiguchi et al., 2008). Quantitative comparison between theory and experiment is influenced by the limited knowledge of the orientation of the molecule in the experiment. Given the combination of channels obtained from shot noise measurements and total conductance, the quantitative agreement is probably better than $30 \%$.

The molecular orientation and the effect of the number of carbon-tip bonds can be observed directly through lowtemperature STM imaging at close tip-molecule distance. Approaching $\mathrm{C}_{60}$ on $\mathrm{Cu}(111)$ by a $\mathrm{Cu}$ coated tip, Schull, Dappe et al. (2011) showed the effect of local bond formation with the tip, which distinguished sites on the molecule having stronger double $\mathrm{C}=\mathrm{C}$ bond character (higher conductance) from those with predominantly single $\mathrm{C}-\mathrm{C}$ bond character (lower conductance). In the case of $\mathrm{C}_{60}$, the conductance is high because the LUMO is triply degenerate and nearly coincides with the Fermi energy of the metal. The DFT computations reproduce different symmetries and bonding sites on a single $\mathrm{C}_{60}$ molecule. However, differences between calculations and measured site-dependent conductance remain, which are partly attributed to a small tilt of the molecules that was not included in the calculations. Quantitatively, despite the high level of experimental information available, a gap of about a factor of 2 remains between experiments and calculations.

In this regime of strongly coupled molecules and high conductance, the quantitative agreement between the conductance in experiments and computations is generally good. The computational accuracy in reproducing the experimental results appear to be mostly limited by details of the experiments that remain unknown. One such effect comes from the scattering of partial electron waves on defects in the metallic leads (Ludoph et al., 1999), which can typically reduce the conductance by $10 \%$, and in exceptional cases even up to $50 \%$. Although more precise testing, for example, by means of 
low-temperature STM experiments, would be desirable, the present evidence suggests that LDA-based calculations in this regime capture the essential ingredients and that the computations have a high degree of predictive power.

\section{B. Low zero-bias conductance}

Before turning to the most widely studied class of molecules, let us first consider the other extreme, the range of extremely low zero-bias conductance. As a practical criterion, we take this to include those single-molecule junctions for which the zero-bias conductance $G(0)$ lies below the measurement sensitivity of the experiments. This limit usually lies at $10^{-5} G_{0}$, but with proper electronics can be extended to $10^{-7} G_{0}$. For those junctions, the molecules would be undetectable by the common histogram-based methods at low bias. Instead, the experimental evidence for the presence of the molecules comes from $I-V$ curves up to high voltage bias. The differential conductance shows a gap of low conductance until one observes a rise, often steep, which may lie at different values for the two bias polarities. If the junction remains stable, one may observe that the conductance reaches a peak value and comes down again at still higher bias.

Figure 29 shows one of the first single-molecule measurements at low temperatures (Reichert et al., 2003) and gives a comparison with data for the same molecule obtained at room temperature. The low-temperature data show a clear gap in the differential conductance until a sharp peak at about $0.5 \mathrm{~V}$ signals the lineup of the chemical potential in the leads with the nearest level on the molecule. The irreproducible fluctuations observed after the peaks are due to instability of the molecular junction under the influence of the high current flowing through the molecule. Comparing these data to the room-temperature curves in Fig. 29(a) reveals several important effects. First, the peaks in the differential conductance become strongly smeared. Second, the gap appears smaller, and the zero-bias conductance is larger at room temperature. These two effects can be understood as resulting from the thermal smearing of the molecular level structure by rapid fluctuations between many configurations. This implies that the position of the molecular levels is sensitive to details, as is further demonstrated with experiments that are discussed next. Obviously, one needs to be careful in interpreting conductance and gap structure from room-temperature data.

The positions of the resonances observed in the differential conductance can be influenced by local electrostatic potentials. Low-temperature experiments using in situ deposition of the metal electrodes (Kubatkin et al., 2003) and experiments using electromigration (Liang et al., 2002; Park et al., 2002; Osorio, O'Neill, Wegewijs et al., 2007) or three-terminal break junctions (Perrin et al., 2013) all demonstrate the sensitivity of the molecular level to the potential applied to a gate electrode. The advantage of a gate electrode is that one can observe this shift continuously as a function of a control parameter, i.e., the applied voltage. However, the actual potential at the molecule will be sensitive to the presence of ions and polar molecules (water) or even dielectrics. Moreover, the image charge distribution in the leads, and therefore the distance of the molecule to the leads and its
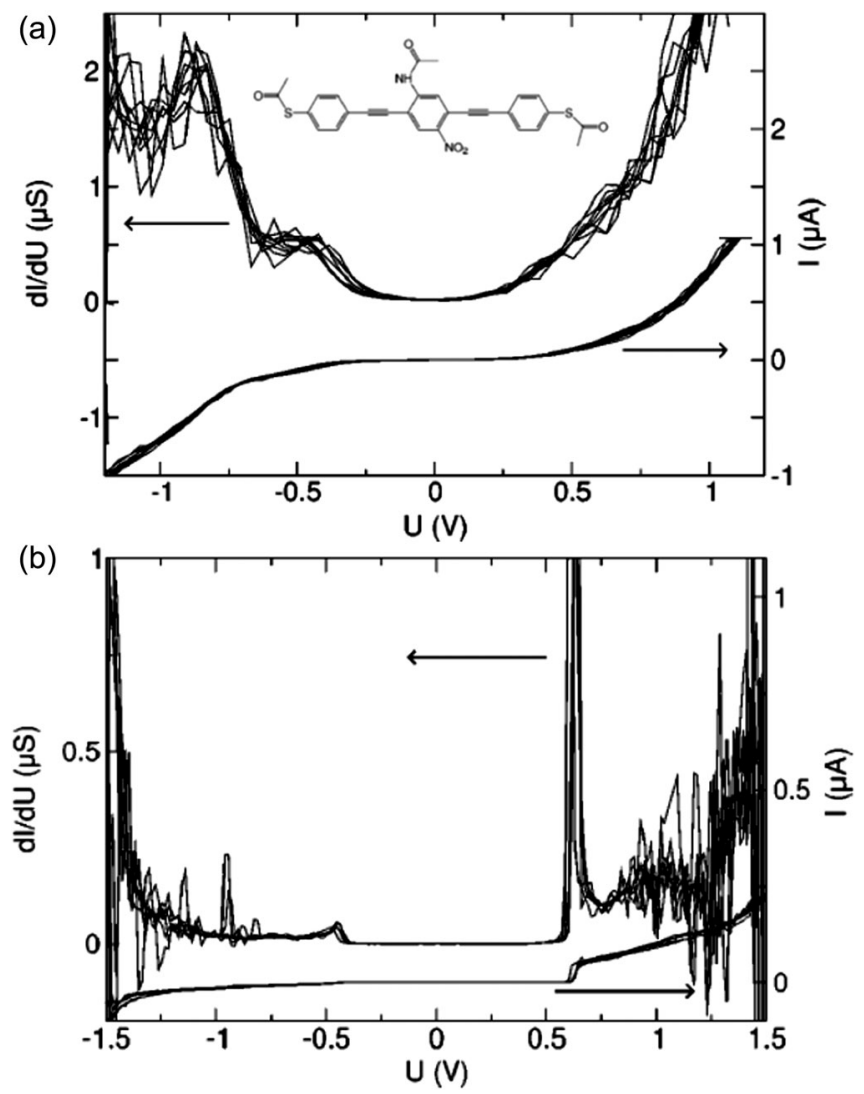

FIG. 29. Single-molecule measurements on a thiol-coupled molecule (shown in the inset in the top panel) having side groups that make it asymmetric. The current (right axis) and differential conductance (left axis) reflect the asymmetry of the molecule. The sign of the asymmetry is random and changes between different measured junctions. The gap in the conductance observed (a) at room temperature become much more pronounced (b) at $30 \mathrm{~K}$. For each curve, several consecutive sweeps are shown. Adapted from Reichert et al., 2003.

orientation, also strongly affects the position of the resonances (Perrin et al., 2013).

Computations for such molecular systems mainly aim at qualitative comparison and cannot easily be made quantitative for several reasons. First, the positions of the resonances are not reliably obtained from DFT-type calculations given the difficulties associated with the theory in predicting proper gap energies. Second, owing to the exponentially small local density of states on the molecule near the Fermi energy, the positions of the resonances are extremely sensitive to the amount of charge transfer between the molecules and the leads. Third, for weak coupling charging effects, which are not easily treated from first principles in DFT, become important. Finally, the calculations must be performed self-consistently under nonequilibrium conditions. While such calculations have been demonstrated for simple systems (Stokbro et al., 2003; Liu et al., 2006; Arnold, Weigend, and Evers, 2007), they are computationally demanding, as discussed in Sec. III.D.4.

Apart from the position of the resonances, the shape of the resonances poses a problem. A straightforward application of resonant tunneling models does not work: the position, height, 
and width of the resonance cannot be simultaneously described by any choice for $\Gamma$; typically, the observed resonance width is by far too large, as shown by the lowtemperature MCBJ experiments by Secker et al. (2011). The conductance at the peak lies invariably much below $G_{0}$, in contrast to the predictions from simple resonant tunneling models. Part of the explanation lies in the role of molecular vibrations. When the resonance lies at low energy (tens of millivolts) clear vibrational sidebands can be resolved, as was also shown systematically by Osorio, O'Neill, Stuhr-Hansen et al. (2007) and Lau et al. (2016). When the resonance shifts to higher energy the vibrational sidebands broaden and eventually cannot be resolved. Yet they continue to determine the full width associated with the resonance. Thus, the width of the resonance is not set by temperature or by the strength of the coupling $\Gamma$ between the molecule and the leads but is approximately set by the reorganization energy due to electron-vibration mode interaction (Secker et al., 2011).

Electronic resonances at still higher energy develop temporal fluctuations, with characteristic times that can be milliseconds (Secker et al., 2011) or much longer (Lörtscher et al., 2006). A typical $I-V$ curve may show a smooth broadened step in current, but zooming in at the step one finds that the smooth transition is actually due to instrumental time averaging of fluctuations of the conductance of the junction, roughly between the values of current before and after the step. The current induces fluctuations in the configuration of the molecule, as was also found for molecules with high zero-bias conductance, where they are triggered by the excitation of vibration modes (Thijssen et al., 2006). As pointed out by Secker et al., given the many additional effects taking place at a resonance the similarity of the experimental curves to those obtained from resonant tunneling models must be regarded as being fortuitous. The broadening of the resonance in the differential conductance may also be taken as evidence that the lattice temperature of the molecule under high bias becomes extremely high.

\section{Intermediate zero-bias conductance}

Having discussed the two extremes, high zero-bias conductance and low conductance, we find that the former type of junctions can be accurately described by computational models, while the latter can be addressed only qualitatively. Let us now review the large group of molecules with intermediate levels of zero-bias conductance, which comprises nearly all molecules studied by means of conductance histograms in break junctions. It goes beyond the scope of this review to present a complete overview of the many molecular systems in this class. Instead, we focus on two widely studies molecular systems, benzenedithiol, a simple aromatic molecular system, and nonaromatic alkanedithiols, which have been adopted as benchmark systems. We propose alternative benchmark systems and conclude with a few general remarks.

\section{Benzenedithiol}

The first electronic transport measurements of a single molecule by Reed et al. (1997) targeted a benzene-1,4-dithiol molecule between two gold electrodes. This system became one of the fruit-fly systems in this field of study. The choice appears to be a natural one because it is one of the simplest systems that offer a fully conjugated path across the molecule. The benzene ring provides a delocalized $\pi$ electron path for conduction, and the sulfur group is assumed to bind covalently to the gold leads and to produce a small electronic barrier between the states of the metal leads and the $\pi$ electrons of the benzene ring. For the zero-bias conductance many different values have been reported, but typically it lies well above the experimental sensitivity threshold, while the differential conductance is strongly nonlinear and shows peaks at high bias. In this sense, the molecule has properties that are intermediate between the two cases discussed previously.

In some ways, the choice of this fruit-fly system is also an unfortunate one because there are few molecular systems for which the experimental results, and there are many, differ so widely. On the other hand, the challenge of understanding this simplest of all aromatic molecular junctions has stimulated many theoretical works and thereby helped to uncover many effects that need to be considered in evaluating molecular junctions. The transmission of the molecule is expected to be high for such a short molecule with a fully delocalized $\pi$-orbital system. However, the measured conductance is extremely sensitive to the details of the connection of the molecule to the Au metal leads, and the short molecule allows for many different arrangements. It turns out that there are many more factors influencing the observed conductance that also have implications for the interpretation of experiments on other molecules. An overview of the experimental data is presented in Table 1 of the Supplemental Material (542), and representative conductance histograms from different experiments are compared in Fig. 30.

The original experiment by Reed et al. (1997) was done in a break-junction setup but did not present any statistical analysis or histogram distributions. Therefore, one cannot judge whether the curves shown in the paper are representative for the Au-BDT-Au junctions. This situation improved after the introduction of statistical analysis based on conductance histograms (Smit et al., 2002; Xu and Tao, 2003). Xiao, Xu, and Tao (2004) were the first to report such measurements for Au-BDT-Au junctions and found a series of peaks in the histogram at multiples of $1.1 \times 10^{-2} G_{0}$ (Fig. 30, top panel). The measurements were done at room temperature at a bias of $0.2 \mathrm{~V}$ in a $0.1 \mathrm{M}$ solution in $\mathrm{NaClO}_{4}$. A systematic study of the bias dependence up to $0.6 \mathrm{~V}$ showed only a weak increase of the conductance with bias, by about a factor of 2 .

This result brought the experimental values much closer to the theory but also led to new confusion. The problem lies, at least partly, in the method of data selection. Most traces of conductance during breaking show only smooth exponential decay. For this reason, Xiao, $\mathrm{Xu}$, and Tao decided to select only those curves that have a distinct plateau region for inclusion in the histogram. The fact that this selection is done manually makes the criteria difficult to judge. The shape of the histogram has not been reproduced by other groups. In fact, a new study by Ulrich et al. (2006), with essentially the same technique, now using 1,2,4trichlorobenzene as solvent, showed that there is no clear structure in the histograms when all curves are included. 


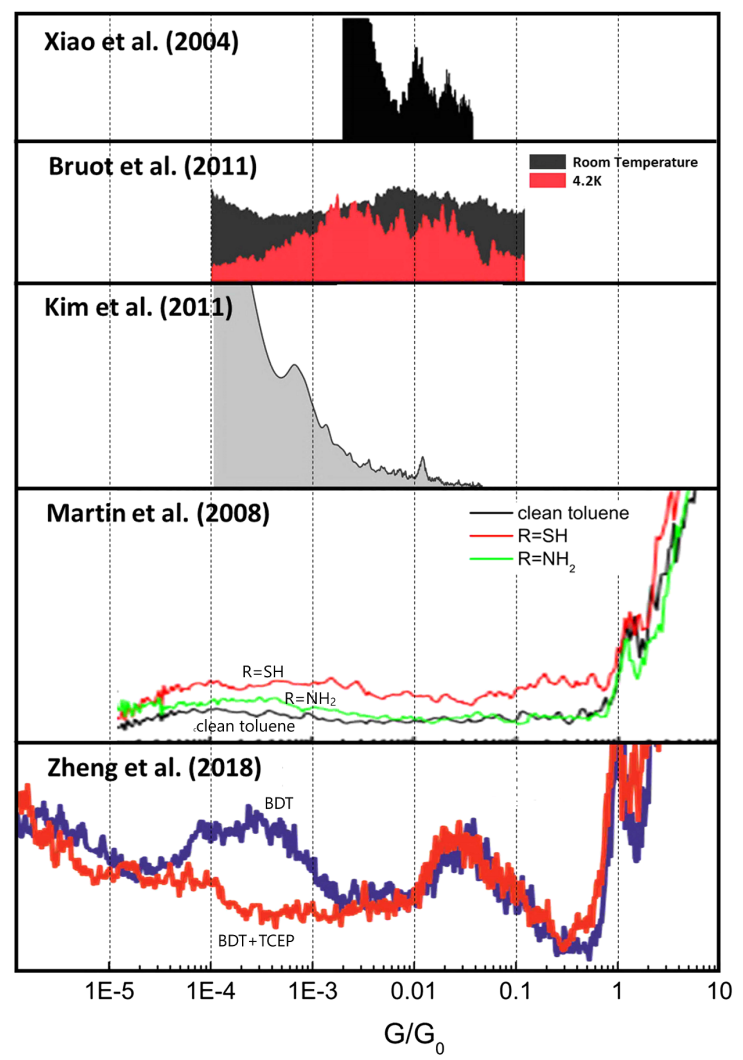

FIG. 30. A selection of conductance histogram data for AuBDT-Au junctions illustrating the wide variation and sensitivity to experimental conditions. The histograms are rescaled for ease of comparison, and have been adapted from Xiao, $\mathrm{Xu}$, and Tao (2004); Martin, Ding, van der Zant, and van Ruitenbeck (2008); Bruot, Hihath, and Tao (2012); Kim, Pietsch et al. (2011); and Zheng et al. (2018). The differences in appearance of the data are due to the choice of the methods of constructing histograms; see also Sec. II.D. Three methods have been applied: (1) First binning data in linear conductance and representing this in a histogram in linear conductance. (2) First binning data in linear conductance and representing this in a histogram in the logarithm of conductance. (3) Binning the logarithm of conductance and representing this in a histogram. The third method is now the method of choice, but earlier data used the other two. The data by $\mathrm{Xiao}, \mathrm{Xu}$, and Tao (top panel) uses method (1) and is mapped onto this logarithmic scale. The data by Kim et al. uses method (2). This enhances the data at low conductance, and the peaks are observed against a decaying background. The other three examples employ method (3).

This conclusion did not change even after implementing an automated data selection procedure, or by taking the experiment to low temperatures $(30 \mathrm{~K})$.

Further work (Fujii, Akiba, and Fujihira, 2008; Martin, Ding, van der Zant, and van Ruitenbeek, 2008; Tsutsui et al., 2008; Kim, Pietsch et al., 2011) confirmed that the presence of the molecules in the junction leads to histogram counts spread over many orders of magnitude, ranging from below $10^{-5} G_{0}$ to several times $10^{-1} G_{0}$. At high bias, above $1 \mathrm{~V}$, Tsutsui et al. observed a broad peak at $0.1 G_{0}$. Different experimental approaches have produced peaks in the histograms for BDT without data selection, although the position of the peaks varies between the methods, suggesting that it is influenced by the choice of experimental method. Using acetyl-protected sulfur groups, Lörtscher, Weber, and Riel (2007) studied the sulfur-coupled benzene molecule by MCBJ under UHV at room temperature and found a single broad peak at $0.5 \times 10^{-4} G_{0}$. Note that the acetyl protection group ideally requires a deprotection agent to be removed to allow direct S-Au bond formation. Since Lörtscher, Weber, and Riel did not report using active deprotection, there is a possibility that the nature of the bond to $\mathrm{Au}$ is different, possibly explaining the low conductance. The question of deprotection is of wider interest and is related to the question of whether or not the $\mathrm{H}$ atom of the thiol group splits off upon bonding to $\mathrm{Au}$, as discussed in Sec. V.C. ${ }^{28}$

Using their $I(t)$ technique, Haiss et al. (2008) obtained for the same acetyl-protected compound a conductance of $1.1 \times 10^{-4} G_{0}$, agreeing within a factor of 2 with the value given by Lörtscher, Weber, and Riel. Figure 30 illustrates the wide variability in the appearance of conductance histograms for Au-BDT-Au single-molecule junctions.

Recently Kaneko et al. (2019) demonstrated the role of different molecule-metal anchoring configurations in the conductance for BDT. They used a self-breaking procedure by which the contact is formed under the influence of spontaneous slow relaxation of the metal leads at room temperature at the last stage of breaking of a junction. This is likely to select only the more stable junction geometries, and they find a high conductance value at $2.4 \times 10^{-2} G_{0}$, a medium value at $3.4 \times 10^{-3} G_{0}$, and a low value at $3.9 \times 10^{-4} G_{0}$. Through simultaneous measurements of surface-enhanced Raman signals of the same molecule, they propose an interpretation for the three conductance states as being due to binding to bridge, hollow, and top sites, respectively.

Theory for BDT.-BDT also played an important role as a model system for benchmarking newly developed $a b$ initiobased transport codes. For this purpose, BDT was suitable because, on the one hand, it is a molecule large enough for it to display typical features of molecular orbitals, while, on the other hand, it is small enough to keep the numerical effort manageable.

First studies of the BDT transmission relied on simplified approaches such as Hückel models (Emberly and Kirczenow, 1998) and DFT studies with electrodes treated as jellium models (Di Ventra, Pantelides, and Lang, 2000). These models were helpful in analyzing the possible role of multiple molecules in the junctions and variations in bonding configurations (Emberly and Kirczenow, 2001). It was realized early on, however, that a proper description of transport of atomic-scale systems, i.e., atomic wires and molecules,

\footnotetext{
${ }^{28}$ For BDT, this question may possibly be decided by analyzing the differential conductance. Stokbro et al. (2003) calculated $I-V$ curves for BDT self-consistently, and the shapes for the $I-V$ curves for the thiol bonded and the thiolate bonded molecules were found to be qualitatively different. The $I-V$ curves for the thiolate bonded molecules show no gap, but a weak local minimum around zero bias at a high value of $0.46 G_{0}$. Instead, for the thiol bond the $I-V$ curve shows a large gap.
} 
requires treating system and contacts on the same footing (Yaliraki et al., 1999; Palacios et al., 2001; Brandbyge et al., 2002); see Sec. III. As a consequence, as time has progressed transport studies have become more elaborate and have included an ever growing number of contact atoms.

To facilitate the extensive calculations, efficient $a b$ initio codes have been developed. The first consistent results for the BDT transmission with Au contacts were obtained by Stokbro et al. (2003), Xue and Ratner (2003), Evers, Weigend, and Koentopp (2004), Faleev et al. (2005), Ke, Baranger, and Yang (2005), Thygesen and Jacobsen (2005b), Grigoriev et al. (2006), Kondo et al. (2006), and García-Suárez et al. (2007). In these studies, minor differences in $\mathcal{T}(E)$ persist, e.g., with respect to the energy position of the $d$-band shoulder; they can plausibly be attributed to variations in the simulation details concerning functional approximations and contact geometries. A systematic study of artifacts related to the finite size of the simulated electrodes was performed by Evers and Arnold (2011).

It turned out that improving the simulation technology was typically associated with an increased zero-bias transmission. This made the perceived discrepancy between DFT-based transport studies and experiments larger, not smaller. Since simulations rely on an ad hoc assumption concerning the junction's atomistic geometry, computational studies have been aimed at investigating the sensitivity of the transmission with respect to geometry changes. As one would expect, at transmissions of order unity strong changes of the binding geometry are required to reduce the transmission significantly. This is because large overlap-matrix elements have to be decimated. Hence, the transmission of BDT turned out to be rather robust against geometry changes, with observed variations of the order of percents (Evers, Weigend, and Koentopp, 2004). ${ }^{29}$

Basch, Cohen, and Ratner (2005) obtained a large conductance of $0.5 G_{0}$ for Au-S-benzene-S-Au binding to a flat Au surface. They also investigated the effect of atomic chain formation, which happens when, upon stretching the junction, $\mathrm{Au}$ atoms are pulled out of the surface and form a connecting chain. In this case, the conductance becomes extremely sensitive to the alignment of the molecule with the chain axis, giving values ranging from nearly perfect transmission down to $3 \times 10^{-4} G_{0}$.

In similar computational studies and assuming a thiolate bond, Sergueev et al. (2010), Borges Pontes et al. (2011), and French et al. (2013) observed that the conductance can vary under stretching nonmonotonically between $0.01 G_{0}$ and $0.5 G_{0}$. In particular, the conductance can increase with stretching, as observed in the experiment by Bruot, Hihath, and Tao (2012).

It is conceivable that at least in some experiments the formation of the molecular junction involves two or more molecules. Investigating this possibility, Strange, LopezAcevedo, and Häkkinen (2010) found a rich landscape of

\footnotetext{
${ }^{29}$ Note that a much larger sensitivity to the binding sites (on top, bridge, or hollow) on a gold surface in the early work by Yaliraki et al. (1999), with variations up to 3 orders of magnitude, is likely due to the approximations used for the coupling to the leads.
}

possible configurations. If sufficient complexity is allowed for, the transmission can vary over orders of magnitude upon stretching.

All in all, the computational studies offer a reasonable interpretation for the wide range of observed conductance values in the experiments. These difficulties possibly appear somewhat more pronounced for BDT than for other molecules because BDT is so short. At this point we conclude that while at present a detailed comparison between theory and experiment appears to be premature the computations nevertheless have uncovered many mechanisms that are likely to simultaneously act in producing conductance values that vary by orders of magnitude, depending on the details of the experimental procedures.

\section{Alkanedithiols}

The second model system that has been widely studied is the series of alkanedithiols (ADT), again coupled between two gold electrodes. We discussed ADT in Sec. V.A, where we were concerned with the systematic variation of the conductance with length. In contrast, here we focus on the absolute numbers of the conductance, their variation between experiments, and quantitative comparison between experiment and theory. In other words, where the focus in Sec. V.A was on the decay constant $\beta$, here we are concerned mostly with the prefactor $G_{c}$.

In contrast to BDT, for the alkanes the backbones of the molecules consist of nonconjugated bonds. Since all carbon atoms are $s p^{3}$ hybridized, alkanes exhibit a band gap in the long wire limit and, thus, are insulating. Therefore, they form an instructive model system and complement nicely to the well studied metallic-atom chains (Agraiit, Levy Yeyati, and van Ruitenbeek, 2003). Before the introduction of single-molecule techniques, many electron transport experiments had already been reported for self-assembled monolayers; see, e.g., Salomon et al. (2003), which we do not cover here. In view of the important role that ADT has played in the initial experimental investigations, we now start with a few historical notes.

Early experiments.-Cui et al. (2001) were the first to contact and measure a single 1,8-octanedithiol (ADT8) molecule. They used a conducting-tip AFM, where the molecule of interest was inserted at low concentration into a dense self-assembled monolayer (SAM) of the monothiol equivalent. Upon exposure of this molecule-covered $\mathrm{Au}(111)$ surface to a solution of gold nanoparticles, the dithiol molecules made a $-S-\mathrm{Au}$ chemical bond with the nanoparticles, and these were probed by the conducting AFM tip. They reported a conductance of $1.43 \times 10^{-5} G_{0}$ at $0.1 \mathrm{~V}$ bias in a toluene solution. Later work from the same group for decanedithiol (ADT10) and dodecanedithiol (ADT12) showed that the conductance of the molecular junctions decreases exponentially with the length of the molecule, and they found a decay constant $\beta$ of $0.58 \pm 0.06$ per $\mathrm{CH}_{2}$ group. This value is about a factor of 2 smaller than found by most groups, which is possibly related to the effect of the dense SAM on the work function.

This may also explain the discrepancy with the work of Tao's group (Xu and Tao, 2003; Xu, Xiao, and Tao, 2003) that followed shortly thereafter. They used their STM-BJ method 
to probe hexanedithiol (ADT6), ADT8, and ADT10 in solution. The conductances for the same wire lengths obtained in these works are more than an order of magnitude higher, and the decay constant $\beta=1.04 \pm 0.05$ per $\mathrm{CH}_{2}$ group is also twice as large, but it agrees well with values known from earlier work on self-assembled monolayers.

On the other hand, the results by Cui et al. received support from Haiss et al. (2004), who used the $I(t)$ and $I(s)$ measurement techniques for probing a similar set of molecules ADT6, ADT8, and nonanedithiol (ADT9). They found conductance values and a decay factor close to those of Cui et al. (2001, 2002). The large discrepancy with the work from Tao's group was investigated by Haiss et al. by repeating experiments under similar conditions. Larger conductance jumps were indeed identified and were tentatively attributed to parallel conduction by gold atom chains. In our view, this is unlikely since $\mathrm{Au}$ atomic chains are not stable under ambient conditions. We return to this discrepancy later. In fact, there is another important distinction between these experiments: whereas Xu and Tao (2003) found a nonlinear dependence of the conductance on the applied bias voltage, Haiss et al. (2004) determined that the conductance scales linearly with biases up to $1 \mathrm{~V}$. This is perhaps the most conspicuous indication that the two experiments are probing different objects.

Overview of experimental results.-In the years since these reports, many groups have measured series of ADT molecules, mainly using different versions of break-junction techniques. All results that we have found are collected in Fig. 31, where the conductance is plotted on a semilogarithmic scale against the length of the ADT molecules, expressed as the number of $\mathrm{CH}_{2}$ groups. Despite the simple character of the molecules, we find that the conductance reported by different groups for each length of the molecule varies by nearly 2 orders of magnitude. On the other hand, the exponential decrease of conductance with length has been confirmed by many works; see the Fig. 31 inset. Nearly all data are consistent with $\beta=0.9 \pm 0.2$ per $\mathrm{CH}_{2}$ group, apart from one exceptional result. This reproducibility, despite the large scatter in the absolute values of the conductance, is associated with the fact that the variation observed in the main panel of Fig. 31 is much smaller if we compare measurements done with the same technique under the same conditions only. To illustrate this, we connect the points obtained from three such studies. The variation between studies illustrates the sensitivity of the conductance to details of the experimental techniques. Note that this poses difficulties when discussing a comparison with computational results because it is not obvious how the experimental conditions affect the data or how this translates into the geometry that theory should consider.

This sensitivity to experimental conditions is highlighted by the fact that several groups reported multiple conductance peaks (shown in different colors in Fig. 31), while other groups reported only a single peak in the conductance histograms. Following Li et al. (2008), we classify the peaks as low $(L)$, medium $(M)$, and high $(H)$ conductance. In fact, each of these three types of peaks often comes as a series of about three peaks at integer multiples of a basic $L, M$, or $H$ conductance. The appearance of such multiple peaks has

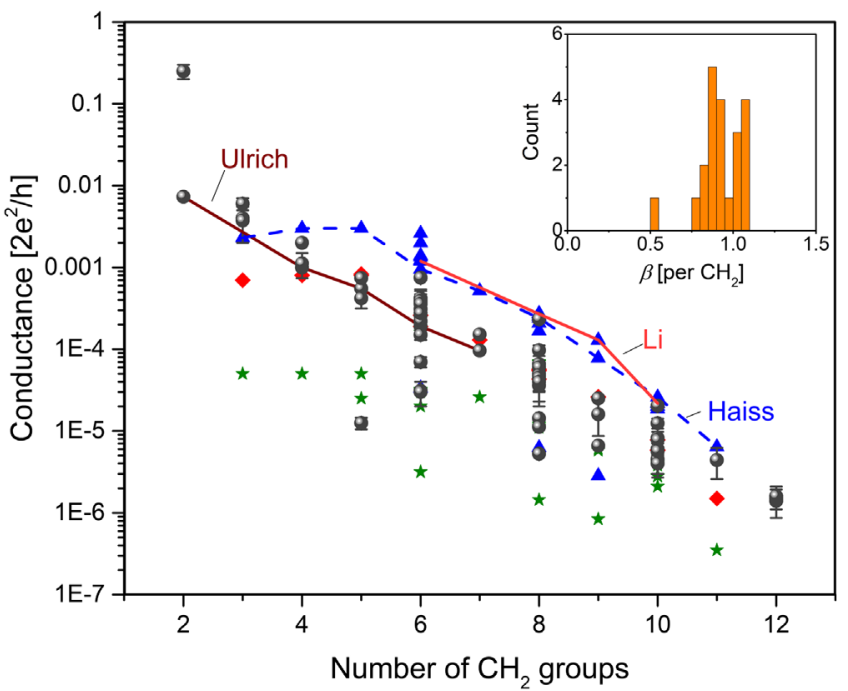

FIG. 31. Overview of measured conductance values for AuADT-Au junctions plotted on a semilogarithmic scale as a function of the length of the backbone in terms of the number of $\mathrm{CH}_{2}$ groups. Symbols are used for distinguishing lowconductance peaks (green stars), medium-conductance peaks (red diamonds), and high-conductance peaks (blue triangles), following the categories introduced by Li et al. (2008). Histograms that show only a single peak and for which the identification in terms of these three categories is not discussed are shown as gray bullets. The data include published work from Cui et al. (2001), Xu and Tao (2003), Xu, Xiao, and Tao (2003), Haiss et al. (2004, 2009)Chen et al. (2006), González et al. (2006), He et al. (2006), Jang et al. (2006), Li et al. (2006, 2008), Sek et al. (2006), Ulrich et al. (2006), Wierzbinski and Slowinski (2006), Huang et al. (2007), Nishikawa et al. (2007), Park et al. (2007), Hihath et al. (2008), Huisman et al. (2008), Kiguchi, Sekiguchi, and Murakoshi (2008), Xia, Diez-Perez, and Tao (2008), Song et al. (2009, 2010)Martin (2010), Arroyo et al. (2011), Kim, Hellmuth et al. (2011), Kim, Song et al. (2011), Rascón-Ramos et al. (2015), Su et al. (2015), and Gil et al. (2018). The spread in the data is large, but the systematic trends in the separate works are shown by connecting the data points obtained from three works, namely, Ulrich et al. (2006), the $\mathrm{H}$ peak from Li et al. (2008), and the H peak from Haiss et al. (2009). This indicates that the decay constant $\beta$ is a more robust and reproducible property, as illustrated for the complete dataset by the histogram in the inset. More complete details on the data collected here are given in the Supplemental Material (542).

been attributed to the formation of junctions with up to three molecules in parallel. Although the details of the experiments differ, all three series of peaks appeared in the works by Li et al. (2008) and Haiss et al. (2009) at similar values of conductance. The experiments are similar in that the tip of the STM is prevented from coming into metallic contact with the metal surface. This is a common feature of the $I(s)$ technique employed by Haiss et al., but Li et al. use a tip approach and retract procedure with a maximum setting of the conductance equal to $0.2 G_{0}$. Perhaps this explains the close agreement between the two datasets, highlighted by the connected points in Fig. 31.

The multiple-peak structure for ADT was reproduced by several other groups; the full dataset is available in the 
Supplemental Material (542). Employing amplifiers with a wide current sensing range, e.g., by using a dual range current amplifier (Li et al., 2008) or logarithmic amplifier, may be decisive for being able to observe multiple conductance peaks. Most standard MCBJ or STM-BJ experiments, which follow a procedure of indentation of the two metal electrodes into metallic contact, produce only a single peak in the conductance histogram. A comparison with the overview of data in Fig. 31 suggests that this peak is most likely associated with the $M$ peak.

The interpretation offered by Li et al. (2008) for the three classes of peaks was based upon extensive DFT model calculations. The conductance in this interpretation was influenced by the bonding configuration of $\mathrm{S}$ on $\mathrm{Au}$ (on top versus bridge-site bonding) and by the presence of gauche conformations in the alkane chain. Although microscopic evidence for this interpretation cannot be obtained from experiment, the model has not been challenged to date. The calculation by $\mathrm{Li}$ et al. agrees with the observed value for the decay constant $\beta \simeq 1$, and the absolute values of the conductance agree within about a factor of 5, assuming that the bonding motifs have been correctly attributed. The variation in bonding configurations and gauche conformations leads to variations in the conductance by about 2 orders of magnitude, which agrees with the spread in the experimental data, suggesting that the details of the experiment influence the averaging process leading to the appearance of peaks in the conductance histogram.

While most of the data reported in the literature agree with the exponential decrease of conductance with increasing length of the alkane chain, with a decay constant $\beta \simeq 1$, the data by Haiss et al. (the blue connected points in Fig. 31) show an anomalous transition to a length-independent regime at $N<5$. This deviation is possibly related to the large bias voltage of $0.6 \mathrm{~V}$ employed in this study.

In hindsight, neither of the popular molecular systems BDT nor ADT is suitable as a benchmark system. Next we discuss a few systems that may be better suited for this purpose.

\section{Alternative benchmark systems}

We present here molecular systems and measurement techniques that offer perspectives as alternatives for quantitatively benchmarking computations against experiments.

Benzenediamine and alkanediamines.-Early on the search began for alternatives to the two previously discussed systems. Venkataraman, Klare, Tam et al. (2006) and Quek et al. (2007) proposed replacing thiol anchors with amine anchors. They were able to show that the peaks in the histograms are much more sharply defined, in particular, for benzendiamine, than for BDT. While this result could not be reproduced in MCBJ experiments in vacuum (Martin, Ding, van der Zant, and van Ruitenbeek, 2008), several other groups found well-defined conductance peaks, reproducing the results by Venkataraman, Klare, Tam et al. to within a factor of 2, for various atmospheres and solvents. An overview of the reported data is given in Table 3 of the Supplemental Material (542).

For alkanediamines [Au $\left.-\mathrm{NH}_{2}-\left(\mathrm{CH}_{2}\right)_{n}-\mathrm{NH}_{2}-\mathrm{Au}\right]$, the number of published results is more limited. While the original data by Venkataraman, Klare, Tam et al. (2006) were confirmed by Chen et al. (2006), in the latter work two sets of peaks were found, one agreeing with the first paper and the second at about an order of magnitude lower conductance. They attribute the appearance of two peaks to different anchorgroup contact geometries.

Quek et al. (2007) presented arguments, supported by extensive DFT computations, for why amines may be more favorable than thiols for producing well-defined peaks in conductance histograms. The main observation was that the overall tendency for the binding of amines to $\mathrm{Au}$ relative to thiols is weaker. As a consequence, the bonding motifs for Au to amine do not vary much for different junction geometries. Furthermore, as a result of the isotropic nature of the $\mathrm{Au} 6 s$ orbital small variations in bond angles and bond lengths would have a limited effect on the conductance.

Despite their promising properties and the fact that amines were proposed as alternative benchmark systems as early as 2006 and that most of the published data support this idea, this proposal has not been followed widely. As we discussed in Sec. V.C.2, the effectiveness of amine anchors may depend on the local environment of the molecules in the experiment. As we see next, the proposed properties for amines may not be unique, and well-defined and reproducible conductance peaks can also be found for thiol-coupled molecules.

OPE3.- There is at least one example showing that thiolcoupled molecules may give sharply defined conductance peaks and highly reproducible conductance values across different platforms. The molecule is the third member of the oligophenylene ethynylenes, OPE3, with S-Au anchoring. Other lengths of this oligomer have also been studied, but less frequently. The molecule is long enough for the properties of the backbone to dominate the conductance over the properties of the anchor groups. Combining the work of 14 experiments from seven independent research groups we find that the conductances of the Au-S-OPE3-S-Au junctions all agree with the value $G=(2 \pm 1) \times 10^{-4} G_{0}$ (Xiao et al., 2005; Huber et al., 2008; Wu et al., 2008; Xing et al., 2010; Kaliginedi et al., 2012; Frisenda et al., 2013, 2015; Wen et al., 2013; Parker et al., 2014; García et al., 2015; Frisenda and van der Zant, 2016; Bopp et al., 2017), as illustrated for a subset of the data from the literature in Fig. 32. Compared to the two widely studied systems discussed previously, the spread in conductance is small. As far as we are aware, the full reason for this reproducibility has not been elucidated. In particular, in view of the sensitivity of the alkanedithiols to top or bridge anchor sites on Au surfaces, the question arises as to why this does not lead to similar spread of reported values for OPE3. Whereas multiple peaks have frequently been reported for ADT, conductance histograms for OPE3 have only a single peak with a maximum in the range $(1-3) \times 10^{-4} G_{0}$.

Although many groups have presented computational results for OPE3, we could not find any systematic studies of the dependence of the conductance on anchoring site, i.e., the choice between top, bridge, and hollow sites. Three calculations that assume hollow-site coupling of the molecule to a flat $\mathrm{Au}(111)$ surface consistently find a conductance for the molecular bridge that is 2 orders of magnitude higher than the experimental values: $0.01 G_{0}$ (Wen et al., 2013), $0.021 G_{0}$ (Paulsson, Frederiksen, and Brandbyge, 2006), and $0.023 G_{0}$ 


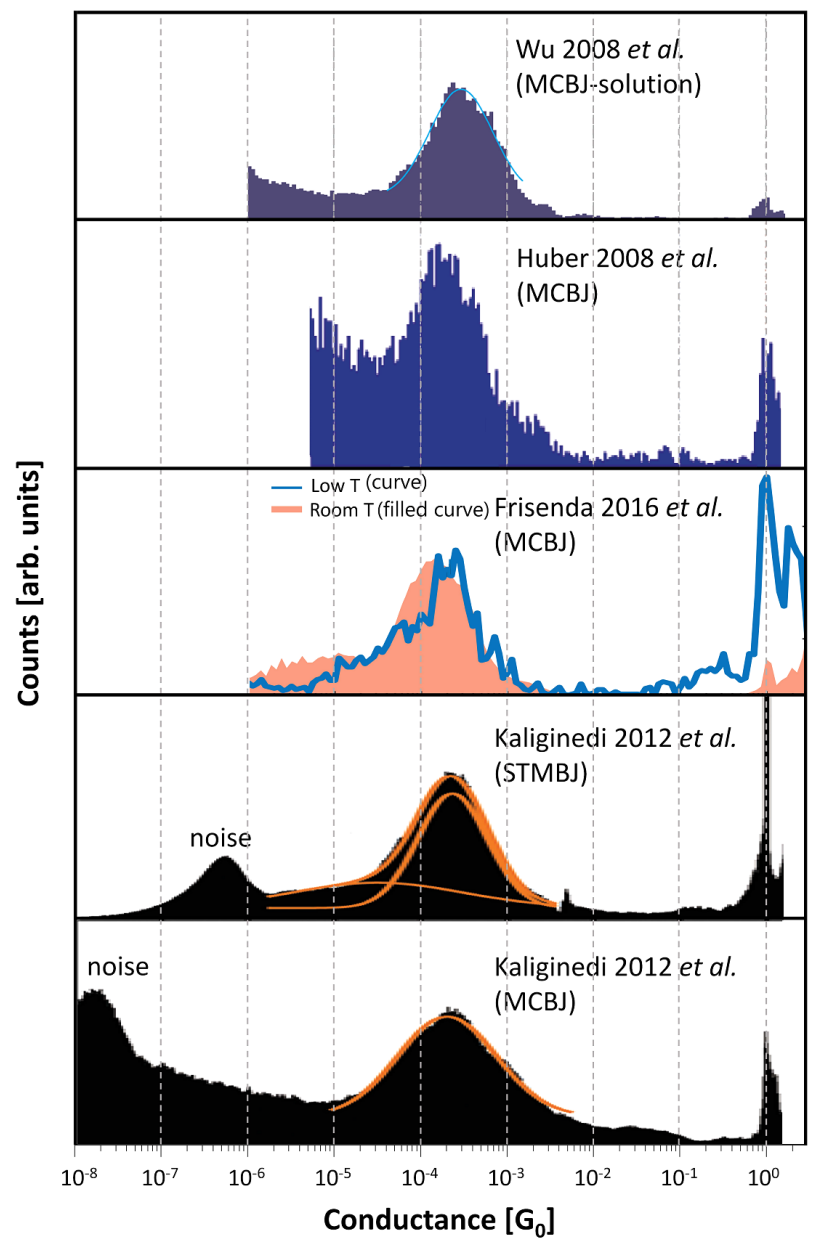

FIG. 32. Comparison of conductance histograms for OPE3 obtained with different experimental methods, under different conditions, and by different experimental groups (Huber et al., 2008; Wu et al., 2008; Kaliginedi et al., 2012; Frisenda and van der Zant, 2016). In all cases, the logarithm of the conductance was used for binning the data into a semilog plot of counts as a function of the logarithm of the conductance. The position of the peak in the conductance reproduces to within a factor of 2 in conductance, and the width of the peak is also fairly reproducible.

(Zheng et al., 2016). Frisenda et al., 2015 calculated the evolution of the conductance during stretching of a molecule bridging two pyramidal Au tips and found a plateau at the final stages, where the binding is to a top site on both ends, of between $1 \times 10^{-4} G_{0}$ and $2 \times 10^{-4} G_{0}$, extremely close to experiment. A partial study of the sensitivity to the binding site given by Kaliginedi et al. (2012) showed a similar value of $4 \times 10^{-4} G_{0}$ for top binding sites, which drops to $0.7 \times$ $10^{-4} G_{0}$ with one anchor moved to a hollow site.

From the experimental evidence we conclude that OPE represents an interesting candidate as a benchmark system, despite the fact that some of the reasons behind the reproducibility need to be elucidated.

Low-temperature STM.-Low-temperature STM experiments offer the best perspectives for benchmarking computational methods. The presence, the identity, the position, and the orientation of the molecule can be obtained from the STM images. The cleanliness of the tip and sample surface can be guaranteed, and the structure of at least one of the two electrodes, the surface, can be known in detail. Although the atomic arrangement of the metal tip is more difficult to characterize in detail, some information on the apex atom is available. While many experimental groups have studied the arrangement and structure of molecules deposited at surfaces, contacting of such molecules by the tip has been addressed by a much smaller community (Joachim et al., 1995; Temirov et al., 2008; Lafferentz et al., 2009; Schmaus et al., 2011; Reecht et al., 2016; Okuyama et al., 2018). Some prominent examples of controlled lifting of a molecule or molecular chain were given by Temirov et al. (2008) and Wagner et al. (2012), and by Lafferentz et al. (2009), Koch et al. (2012), and Reecht et al. (2015). However, the systems studied in these works introduce additional complications for quantitative comparison with computations due to correlation effects in the former case (as discussed in Sec. V.I.3), and the many molecular conformations of the long chain during lift-off in the latter case.

A prime example of an experiment designed for close comparison with theory was given by the Berndt group (Jasper-Tönnies et al., 2017); see Fig. 33. The molecule chosen for this study, propynyltrioxatriangulenium, has a tripod structure designed for resting on the $\mathrm{Au}(111)$ substrate, with a propylyl wire protruding straight up from its center. Approaching the center of the molecule with the tip from above, Jasper-Tönnies et al. observed a variation of the conductance, which they interpreted with the help of DFTNEGF computations as follows: as the tip comes closer to the top methyl group, the latter is repelled and the propylyl wire bends to the side. Approaching further, the bent molecule exposes the carbon triple bond to the apex atom, to which a bond is formed, as is revealed by a jump of about an order of magnitude in the conductance. At this point, the conductance is $3.5 \times 10^{-3} G_{0}$ and this conductance varies by less than a factor of 1.4 between experiments with different tips and different molecules. This example illustrates the complementary type of information obtained from theory and experiment and a close agreement between the qualitative features. From this agreement, we gain confidence in the information that DFT provides about the nature of the molecular orbitals involved, about the change in couplings as a consequence of mechanical deformation, and on the size of the molecular deformation that is induced by the tip. Quantitatively, the computational results for the conductance even agree to within a factor of $\sim 4$. The DFT calculations include longrange dispersion interactions but have not been adjusted by scissor operators for the overestimation of the HOMO-LUMO gap or for image-charge shifts.

\section{Concluding remarks}

While our qualitative understanding of electron transport in molecular junctions has reached a high level, as we concluded in Sec. V, the quantitative agreement between experiments and theory is often still not firm. This overview shows that many unknowns hamper a proper comparison. The reason why we stress this is that interesting physical effects may be overlooked if we cannot make a proper comparison. The problem resides both with experiment, where the choice of proper 

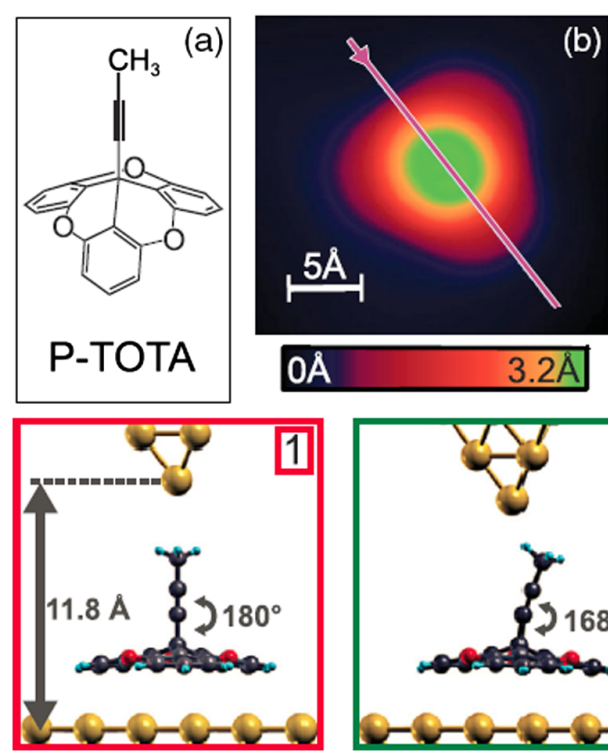
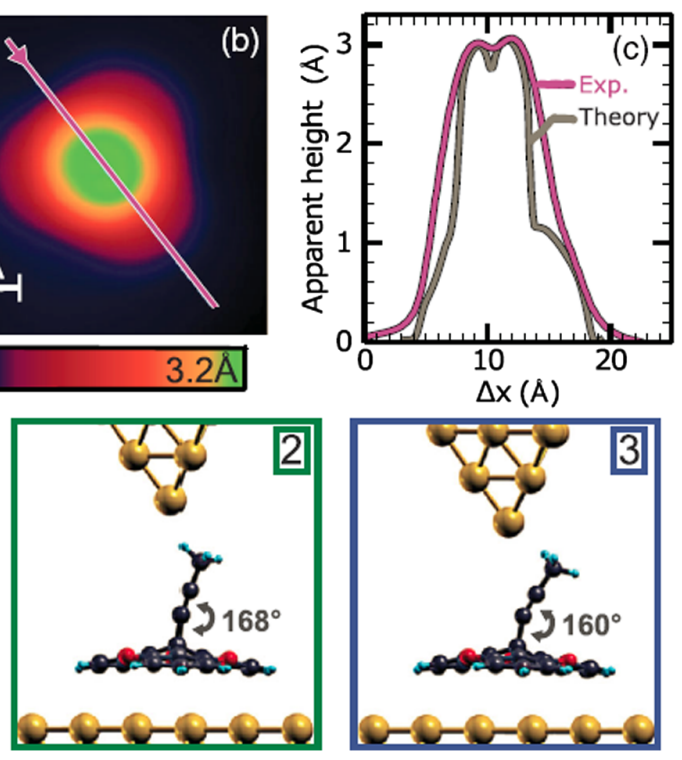

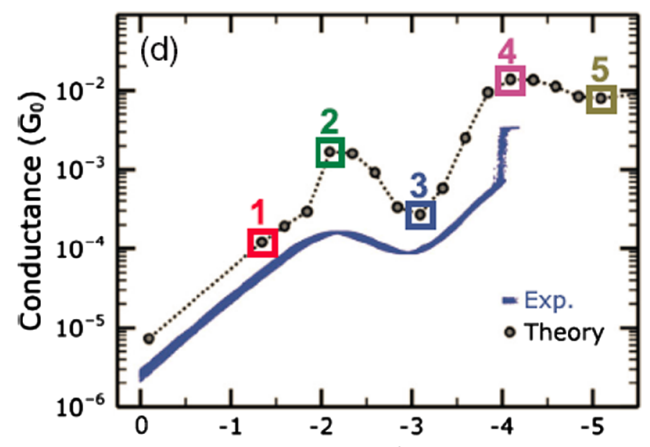

$\Delta \mathbf{z}(\AA)$
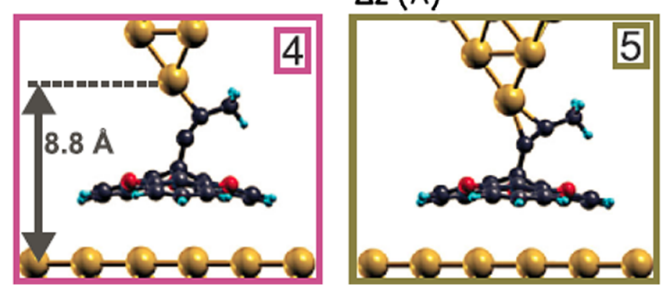

FIG. 33. STM experiment on the tripod molecule propynyltrioxatriangulenium (P-TOTA) and a comparison with computations. (a) Structure of the molecule. (b) Height profile of a single P-TOTA molecule as observed in a STM image recorded at $4.5 \mathrm{~K}$ for a $100 \mathrm{mV}$ bias and at a $30 \mathrm{pA}$ current set point. (c) Height profile along the trajectory indicated in (b) and compared with computations. (d) Conductance-distance dependence for approach of the tip to the center of the molecule from above. The experimental curves are shown for 40 forward and backward traces. The configurations of the molecule in the computations for the positions of the tip at the numbered stages are shown in the lower panels. From Jasper-Tönnies et al., 2017.

benchmark systems has not yet been made, and with theory, where the limitations of DFT become apparent in the treatment of the HOMO-LUMO gap, in the effects of image charges in the leads, and in describing electron correlations.

As has been put forward by several groups, quantitative comparison becomes much better when considering ratios of experimental data [see, e.g., the switching of conductance for two magnetic states of a molecule (Schmaus et al., 2011) or a comparison of different connection sites on a molecule, as in the "magic ratios" discussed by Geng et al. (2015) and further reviewed by Ulčakar et al. (2019)]. The results for ratios compare quantitatively because many of the aspects of the theory that are sensitive to matrix elements drop out.

Despite the remaining gap in quantitative agreement mentioned previously, the role of DFT-based computations in guiding and interpreting the experiments should be strongly emphasized. Given the limited number of parameters that experiments typically give access to, computations complement the experiments and guide our understanding. In situations where the conductance of a single species is the only parameter available for comparison and the atomic structure is not fully known, we must remain cautious to avoid misinterpretations.

\section{SELECTED OPEN PROBLEMS}

Successes and challenges of modeling and understanding the transport properties of molecular junctions have been addressed throughout this review. Here we address a few specific examples of open questions that have not been fully resolved. These examples help to illustrate the prospects for further research and discoveries.

\section{A. Experimental phenomena awaiting basic qualitative understanding}

Arguably the most interesting open problems concern strong experimental signatures that are awaiting a consistent qualitative explanation. As an example, we describe recent work by Frisenda and van der Zant (2016) on an OPE3 molecule equipped with thiol linkers coupled to $\mathrm{Au}$ leads. They found a sudden transition from a smooth conducting state into a peculiar insulating state, driven by subtle stretching of the molecular junction; see Fig. 34. Frisenda and van der Zant favored an interpretationin which the transition is one into an emergent insulator regime dominated by strong correlation effects of the Coulomb-blockade type. This is a conspicuous result, and the mechanism invoked calls for further investigation. On the experimental side, can we exclude alternative explanations? Can we exclude the fact that the state of binding of the molecule changes under the influence of the combination of stretching and high voltage bias? Can we find further evidence for such a sudden change of the character of the electron transport, e.g., by probing shot noise?

Other explanations are conceivable (Frisenda and van der Zant, 2016), such as formation of a polaron or a change of the redox state, and these can be further explored by experimental and theoretical investigations.

\section{B. Chirality-induced spin selectivity}

An entire class of phenomena was discovered experimentally by Ron Naaman, Dave Waldeck, and co-workers. Early measurements employed photoemission of electrons from $\mathrm{Au}$ surfaces, where the electrons were transmitted through films 

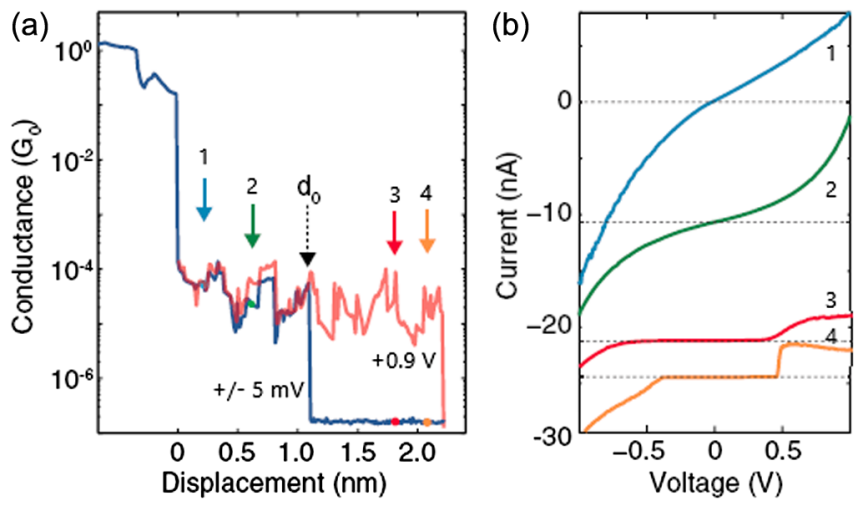

FIG. 34. Observations of anomalous behavior for an OPE3 molecule coupled by thiol bonds to Au in MCBJ experiments at $10 \mathrm{~K}$. (a) Traces of conductance recorded while breaking the molecular junction, shown for low bias [obtained from the slope of $I(V)$ around $V=0$ ] and high bias voltage (obtained from the ratio $I / V$ at $V=0.9 \mathrm{~V}$ ). While the two curves nearly coincide up to the point marked as $d_{0}$, at this point there is a sudden break in the curve for low bias voltage, while the high-bias curve for the same junction continues without interruption. (b) Current-voltage curves shown for the four different electrode separations marked by the colored arrows in (a). A vertical offset is applied for clarity. Following a smooth evolution of $I(V)$ at lower electrode separation, a sharp transition is seen to a gapped state. From Frisenda and van der Zant, 2016.

of chiral molecules (double-stranded DNA) adsorbed on the $\mathrm{Au}$ surfaces (Ray et al., 2006; Göhler et al., 2011). These works strongly suggested that the interaction of the spin of the transmitted electrons with the adsorbed layer is extremely sensitive to the molecular handedness. By now an impressive body of experimental work has been compiled revealing the presence of "chirality induced spin selectivity" (CISS) under diverse conditions, including single-molecule electron transport experiments (Xie et al., 2011); for a review, see Naaman and Waldeck (2015). As was suggested recently, CISS may have important technological applications as a novel method for enantiomeric separations, which is important in pharmaceutical production processes (Banerjee-Ghosh et al., 2018).

Despite considerable investment in theoretical work, there is no consensus yet as to the fundamental origin of CISS. In principle, there are two conceivable ways by which molecular handedness could couple to the electron's spin, producing a CISS effect: (i) a mechanism mediated via spin-orbit coupling (SOC), and (ii) a mechanism operating in the presence of a current flow that translates handedness into an induced magnetic flux. At present all mechanisms invoked to explain strong CISS seem to fail, underestimating the observed magnitude significantly (Naaman and Waldeck, 2015).

Because of its prominent character and potential technological impact, we offer a brief survey of the theoretical attempts to understand the CISS phenomenon in terms of technology and basic science. Earlier theoretical treatises motivated by transmission electron measurements (Ray et al., 2006; Göhler et al., 2011) addressed CISS in terms of scattering approaches (Yeganeh et al., 2009; Medina et al., 2012; Eremko and Loktev, 2013; Gerstin, Kaasbjerg, and Nitzan, 2013; Varela et al., 2014). Since our focus is on electron transport, we refrain from following that direction here.

CISS induced by SOC on the molecule.-A considerable number of theoretical works on CISS report transmission calculations for various tight-binding models (Guo and Sun, 2012a, 2012b, 2014; Gutierrez et al., 2012; Guo et al., 2014; Varela, Mujica, and Medina, 2016). Gutierrez et al. (2012) considered a single-channel tight-binding model with nearestneighbor hopping and SOC. They motivated their model parameters referring to DNA; the hopping parameter is reported to take values of $20-40 \mathrm{meV}$. Chirality enters the model indirectly via its feedback into the SOC. To find quantitative estimates of the latter, a heuristic argument is exploited that yields typical values for light atoms $(\mathrm{C}, \mathrm{B}, \mathrm{N}, \mathrm{O})$ of about $2 \mathrm{meV}$ coupling strength. At present the accuracy of this estimate is not known. Indeed, scales of meV can be reached with light elements, e.g., when promoting a carbon atom in graphene from $s p^{2}$ to $s p^{3}$ hybridization. However, compared to this promotion, chirality-induced symmetry breaking should be weaker by a geometric factor. Therefore, the reliability of the heuristic estimate appears to be uncertain.

Guo and Sun (2012b) also considered double-stranded wires within a tight-binding description. Guo and Sun (2012a, 2012b, 2014) and Guo et al. (2014) employed model parameters similar to those given by Gutierrez et al. (2012) so that the quantitative uncertainties would carry over.

Symmetry constraints on spin filtering in two-terminal transport.-Guo and Sun (2012b) and Matityahu et al. (2016) emphasized the importance of two channels for the observation of CISS. Indeed, it is well known that the SOC can be gauged out in single-channel wires, so spin filtering functionality based on SOC is not expected (Meyer, Falko, and Altshuler, 2002). In noninteracting single-channel wires, one can also make an argument based on time-reversal symmetry according to which a single-channel wire can never act as a spin filter in a two-terminal measurement (Kiselev and Kim, 2005; Bardarson, 2008). Time-reversal symmetry also has implications for the conductance of interacting wires with several channels. In this case, an Onsager type of symmetry relation holds that inhibits spin filtering in two-terminal measurements (Yang, van der Wal, and van Wees, 2019).

Nonunitary effects.-A conceptually innovative workaround for no-go theorems was put forward by Guo and Sun $(2012 b, 2014)$ and further explored by Matityahu et al. (2016, 2017). They investigated the effect of a third bath that the electrons traversing the chiral molecule may be coupled to. The bath gives rise in general to nonunitary effects such as dephasing and leakage. Technically, this effect can be modeled by a complex self-energy that can bring about spin-selective transport in the presence of spin-orbit interactions; intuitively, evanescent waves associated with opposite spins have different decay lengths. The overall magnitude of the filtering effect thus brought about appears to be too small explain the main experimental features observation.

CISS induced by substrate mediated SOC.- Since straightforward SOC on the chiral molecules is suspected to be too weak to account for the experimentally observed magnitude of CISS, it is natural to consider the role of the substrate. In most experimental situations, this substrate is taken to be a $\mathrm{Au}$ 
surface, where SOC can be considered sizable. Indeed, Gerstin, Kaasbjerg, and Nitzan (2013) predicted that a considerable spin polarization can be obtained by a mechanism that combines strong SOC in the substrate with orbital angular momentum selectivity imposed by the chiral molecule.

Outlook.-At present it appears that a large gap remains between the experimental observations and the quantitative estimates from theory. Currently theoretical investigations are focusing on qualitative aspects, e.g., on rigorous bounds set by symmetries, such as time reversal (Dalum and Hedegård, 2019; Yang, van der Wal, and van Wees, 2019). Further experiments are required for guiding the theory and for limiting the possible interpretations for this potentially important phenomenon.

\section{Challenges to theory and modeling}

One out of several important challenges for theory is achieving systematic, quantitative control of accuracy in electronic-structure predictions for molecule-metal interfaces. We illustrate this point with two specific examples.

"Unphysical" values for fitted model parameters.-Perrin et al. (2014) measured large NDR effects in a thiolated arylethynylene with a 9,10-dihydroanthracene core between $\mathrm{Au}$ electrodes. Suggested by the molecular geometry illustrated in Fig. 35, the differential conductance was modeled by a TLM (see Sec. IV.A.1), where the two levels represent the left and right conjugated arms, separated by a nonconjugated linker in the middle. The experimental line shape reproduces well on a qualitative level including, in particular, the strong NDR feature, even though interactions are not accounted for. However, quantitative agreement is achieved only after rescaling the model curve by a large factor of $7.2 \times 10^{-5}$.

We suspect that the large prefactor suggests the importance of correlation effects beyond the noninteracting TLM. The situation is not untypical in the sense that fitting formulas resulting from noninteracting theories often lead to matching fits at the expense of choosing fitting parameters that are hard to justify physically without invoking strong, interaction mediated renormalizations. In a way, we encounter a molecular analog of the Fermi-liquid theory. How to calculate the corresponding "Fermi-liquid corrections" quantitatively is an important open question in molecular-scale electronics and neighboring fields.

Quantitative DFT calculations.-The lack of control in $a b$ initio transport calculations is best illustrated by recalling the specific problem of alignment between molecular levels and

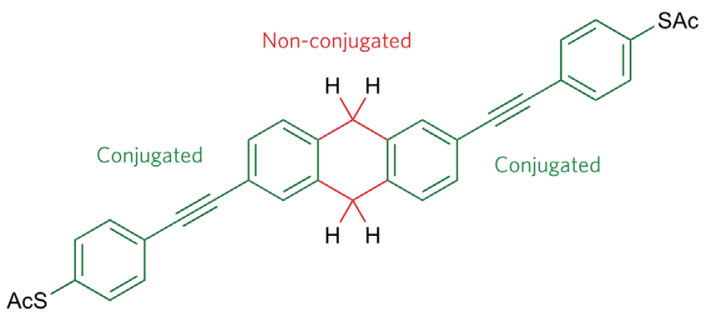

FIG. 35. A thiolated arylethynylene molecule with a 9,10dihydroanthracene core (the three rings in the center). The core divides the molecule into two conjugated parts. the Fermi energy associated with metallic substrates. The problem is severe enough, leading sometimes even to qualitative discrepancies.

For example, Wang et al. (2009) studied the length dependence of the conductance of oligoyne molecular wires $(N=1,2$, and 4 units). The experimentally measured conductance drops by roughly a factor of 2 with increasing length. Inconsistent with this, DFT calculations employing the LDA functional yield a weak increase of the conductance. As Wang et al. argued, the discrepancy is due to an insufficient accuracy of the semilocal functional employed in the DFT study.

One of the most important defects of such semilocal functionals is the neglect of the derivative discontinuity with the associated mistreatment of the Coulomb blockade. Inaccuracies with respect to the Kohn-Sham energy-level alignment are an important consequence. As we explain in Sec. III.D.3, some have adopted an ad hoc recipe (scissors operator) as a post-DFT repair treatment and thereby achieved more quantitative conductance values (Quek et al., 2007, 2009; Mowbray, Jones, and Thygesen, 2008). After following this procedure, a match of the trends between theory and experiment was restored by Wang et al. (2009).

A proper treatment of the Coulomb blockade in intermediate-size systems has been a significant challenge for electronic-structure calculations in quantum chemistry and material sciences ever since such calculations have been attempted. While correlated methods are under continuous development, e.g., based on many-body Green's functions, their application to quantum transport remains challenging, in particular, due to computational issues.

\section{CONCLUSIONS}

The confrontation between theory and experiment that we have presented leads to several important conclusions.

\section{A. Benchmark systems}

For a critical evaluation of comparison between theory and experiment it is convenient to distinguish three classes of molecular junctions, although admittedly these classes are not sharply defined. The first class, comprising small molecules with a conductance close to $1 G_{0}$, is well under control. For this class, experiments give access to many additional tools for characterization (shot noise, thermopower, inelastic electron tunneling spectroscopy, etc.) that often allow for simple interpretations and that assist the testing of theoretical models. It turns out that the model calculations in this limit are less sensitive to details of the electrode-molecule interface, and close quantitative agreement between theory and experiment is obtained. Although sometimes there may be a factor of $\sim 2$ between the experimental conductance and the value obtained in calculations, we feel that most of the discrepancy can be attributed to atomistic details in experiment that are not included in the models.

The second class covers the other extreme, including molecular junctions having extremely low conductance, here defined as having a zero-bias conductance below the detection limit of the experiment. For this class, comparison between 
theory and experiment can be made on a qualitative level only. This is because in this limit experiment and theory alike are sensitive to details. Moreover, comparison can only be made for current levels at rather high bias, i.e., at strongly out-ofequilibrium conditions, where the approximations underlying $a b$ initio transport calculations are the least controlled. Nevertheless, many interesting phenomena have been uncovered for such junctions.

Finally, the third class includes molecules falling between these limits, which comprises the majority of molecular junctions studied to date. Contained in this set are two types of molecules that have played the roles of benchmark systems from early on, namely, BDT and the series of ADT. BDT has served an important role as a workhorse in computational studies, where the results in essence agree between research groups, but it has been an unfortunate choice in terms of experiments. There is a large variability between various experiments, suggesting that the preferred binding configurations of the BDT molecule between metal leads depend on details of the experimental procedures. ADT has the advantage over BDT of offering the possibility of studying a trend, i.e., the length dependence of the conductance. The systematics of this trend is well defined and reproducible in experiments and understood quantitatively. Contrasting with this, a large variability of the experimentally measured contact conductance is observed for ADT, which appears to be consistent with the findings for BDT.

As an alternative benchmark system for comparison between experiment and theory, we propose giving a more prominent role to oligo(phenylene ethynylene)dithiol. It appears that this molecule in the three-monomer long version (OPE3) has been studied by many groups with different methods. The conductance values and the shape of the conductance histogram were found to be highly reproducible. At this moment it is still unclear why OPE3 does not suffer from the same problems as BDT and ADT. Two additional reasons for studying this molecular system more closely are (i) the fact that the experimental and theoretical conductances still differ by 2 orders of magnitude, and (ii) the observation of anomalous behavior at low temperatures, as discussed in Sec. VII.A. Other oligomers have also been studied (Lu et al., 2009; Xing et al., 2010; Kaliginedi et al., 2012; Zheng et al., 2016), but not yet by many groups or methods. It is likely that many other suitable systems exist that would provide closely reproducible conductance values.

\section{B. Uncovering physical phenomena with robustness}

Despite many atomistic unknowns existing in single-molecule junctions, a great number of phenomena have been uncovered and understood. Such phenomena are often associated with a sense of "robustness" with respect to the physical observable. Depending on the observable, the origin of this robustness varies and different mechanisms have been discussed in this review. For instance, in dimensionless ratios of observables, such as the magnetoresistance, prefactors strongly fluctuating from junction to junction cancel out. Similarly, when analyzing trends the relative conductance (taking the typical value) often is reproducible between different experimental settings, while the absolute values may not be. The experimental observation of destructive quantum interference (DQI) has strongly benefited from this kind of robustness. Indeed, the fact that simple counting rules have been found predicting DQI reliably irrespective of chemical details of the anchor groups can be understood as a manifestation of this robustness. Ultimately, it originates from the stability of the nodal structure of the frontier orbitals.

We discuss conditions of robustness with an eye on breakjunction experiments. When a series of molecules are studied with a systematic variation in a single parameter (e.g., its length), one may expect that this single parameter dominates the trend in the conductance provided that the experiment meets at least the following conditions: (a) the contactbreaking procedure for all molecules under test must be performed following the same protocol, (b) the core of the molecule must dominantly determine the value of the conductance, and (c) the dominant anchoring configurations are not influenced by the parameter that is varied between the molecules. For predictions, DFT studies can be helpful in evaluating whether (b) and (c) are likely to be fulfilled.

A completely different, genuinely many-body mechanism of robustness is realized in the Kondo effect: the Abrikosov-Suhl resonance is always situated at the Fermi energy, so the existence of a zero-bias anomaly is guaranteed as long as there is a free spin. Since the free spin does not require fine-tuning, the Kondo effect is a generic encounter in open-shell molecules. As we discuss in this review, metal-organic molecules turn out to be an ideal test bed for studying Kondo physics, e.g., its dependency on space and continuous parameter tunings, or in the presence of competing spins and several channels. Indeed, the elusive phenomenon of the underscreened Kondo effect was first observed in such systems.

Robust are also many observed effects that relate to the coupling of electrons to vibrations, such as the FranckCondon blockade. For this reason, molecular junctions have offered a solid platform for investigating inelastic signals in the differential conductance and in noise, and the way these depend on the electron transmission probability of the junction.

\section{The important role of DFT-based computations}

Experiments provide access to only a limited number of observational parameters, in many cases only the value of the conductance averaged over many junction-breaking cycles. This fact has led to some overemphasis on the numerical value of the conductance. More important than the conductance value itself are the predicted qualitative features and trends. We recall that DFT serves as a guide to the experiments, where it may indicate which are the more energetically favorable bonding configurations, which molecular orbitals are likely to dominate the electron transport, and which symmetries apply to these electronic states. Moreover, trends in the experimental transport data are often closely reproduced and are expected to be more reliably predicted, as shown by Schmaus et al. (2011) and Geng et al. (2015).

However, there are cases, and we have discussed a few, where the discrepancy is larger and trends extracted from DFT-based transport calculations fail qualitatively. This indicates the presence of physics ignored in DFT, at least when 
employing conventional exchange-correlation functionals. Extensions may need to consider dynamics of image charges, strong electron-electron correlations, polaron formation, and other many-body effects.

We emphasize that even in cases where the actual physics is not contained in DFT-based transport simulations a wellinformed setup of DFT-calculations can, and normally will, provide good guidance. By computing estimates for the level broadening $\Gamma$, for the molecular charging energy $U$, for the vibration frequencies and coupling matrices, etc., it is possible to decide in which corner of the physical phase space the system finds itself. To provide an example, we consider the Kondo effect. It is not captured by the available DFT functionals. However, an open-shell calculation even with conventional functionals can indicate the existence of a free spin. This observation then will be readily interpreted as a necessary (i.e., mean-field-type) precursor for the Kondo effect, which would be seen if more accurate tools were available.

\section{Outlook}

The field of single-molecule transport can be seen as an active subfield of the much larger research area molecularinterface sciences. As we emphasize in the Introduction, only aspects of it could be covered in this review. Similarly, also this outlook reports only selected emergent directions chosen from many promising ongoing activities worldwide.

\section{Precision, reproducibility, and control}

Quantitative electronic-structure calculations of interfaces between molecules and condensed matter, such as metals and semiconductors, will remain a challenge for the foreseeable future. Moreover, despite shortcomings of DFT, trends can be predicted and designs can be optimized, even quantitatively. To facilitate such predictions with a perspective of high throughput, a work-around can be designed following a trend in materials sciences that replaces $a b$ initio-based understanding by large data fitting: computations merely extrapolate between experimentally secured data "points." Such a data point consists of structural information, e.g., type and length of molecular wire, anchor group, electrode material and distance, etc., together with a measure that determines "distances" in this configuration space. Further qualifying information includes parameters observed in transport experiments, e.g., typical conductance, Seebeck coefficient, forces, etc. "Secured" implies that the data collected in the data point satisfy a set of quality criteria, in particular, reproducibility. With increasing size of the secured dataset, the number of fitting parameters that are being used to parametrize the extrapolating functionals also grow, which may call for parametrization techniques such as machine learning.

Reproducibility can be established in a statistical sense as a property of a series of measurements. The production of secured data sets with individual reproducibility not only requires a precise measurement of electronic-structure features but also needs to specify the corresponding atomistic geometry. Arguably the best prospects in this direction offer low-temperature scanning tunneling and atomic force measurements. Such studies will turn out to be crucial not only in the subfield of single-molecule transport but also from the broader perspective of molecular-interface sciences.

\section{Toward novel phenomena: Challenges for experiments}

We cover in the review a number of theoretical developments that are well advanced, e.g., the formation of polarons in soft molecules or the Kondo effect in systems with two coupled spin degrees of freedom. In these situations, the theory is awaiting experimental confirmation. Further theoretical investigations are ongoing, with good prospects for experimental testing.

Many-body quantum interference.-QI in single-molecule transport has been a topic of intensive research in the past decade, with a strong emphasis on single-particle phenomena. Much less is known for situations where states interfere that consist of a few Slater determinants instead of just a single one. One would expect that many-body QI should be a frequent encounter in molecular systems, but so far it has been identified only in exceptional cases (Yu et al., 2017).

To give a perspective, we mention that many-body interference is a topic closely related to ongoing research directions in neighboring fields. For instance, in condensed matter physics the quantum interference of many-body states drives many-body localization (Nandkishore and Huse, 2015; Bera et al., 2017). The phenomenon is associated with a breakdown of diffusion in inhomogeneous wires that can persist even at elevated temperatures, provided that the coupling to the environment and vibrational modes is sufficiently weak. To what extent single molecules could be a suitable test bed to study this fascinating phenomenon remains to be seen.

Light-matter interaction.-In many fields of science, light offers powerful tools for investigation. For example, advanced spectroscopic tools have been developed that are capable of interrogating individual molecules embedded in an isolating matrix (Orrit, Ha, and Sandoghdar, 2014). For molecules connected to metallic leads, fluorescence is quenched by electron-hole excitations in the leads. The metallic leads similarly hamper electroluminescence and other forms of light emission and absorption. For example, quenching of optical excitations of the molecule plays an important role for achieving light-induced switching in single-molecule junctions (Dulić et al., 2003; van der Molen et al., 2009).

In contrast, there is one form of light-matter interaction that profits from the proximity of the metallic leads, which is Raman spectroscopy (Ward et al., 2008; Natelson, Li, and Herzog, 2013; Iwane, Fujii, and Kiguchi, 2017). In this case, the light excites surface plasmon modes in the metallic tips, which lead to an enhanced electric field at the molecule. Other forms of light-matter interaction can be studied by separating the active center in the molecule from the metal by insulating layers, or insulating molecular wires. For this reason, light emitted from single-molecule junctions has been seen first after separating the molecule from direct contact with the metallic leads (Qiu, Nazin, and Ho, 2003; Marquardt et al., 2010; Reecht et al., 2014; Doppagne et al., 2018).

Molecular wires.-Quite generally, long molecular wires open interesting perspectives for studying new effects related to strong electronic correlations and topology. For instance, polymethines can form topological insulators realizing the 
Su-Schrieffer-Heeger model (Heeger et al., 1988). Indeed, first indications of topological edge modes in single molecules have been reported only recently by Gunasekaran et al. (2018) and, in particular, by Gröning et al. (2018). Much more is to be expected here to come, such as studies on the formation of topological defects ("solitons") and their propagation inside the wire. In wires that are closer to a metallic state, strong correlation effects are expected including spin liquids. As a relatively simple example illustrating the prospect, we mention the oligoacenes; they represent a research field in their own right, with a respectable body of literature, because they might exhibit a correlated phase (Shen et al., 2018). It has been proposed that oligoacenes might exhibit a band gap oscillating with increasing wire length (Schmitteckert et al., 2017); see van Setten et al. (2019) for a recent overview.

Molecular Kondo chains.-Since single-molecule Kondo effects are abundant in the literature, a natural next step would be to take a look at molecular Kondo clusters and networks interfacing a normal metal. While reports of such systems exist, deviations from the single-molecule Kondo behavior are rare (Tsukahara et al., 2011; DiLullo et al., 2012; Fernández, Aligia, and Lobos, 2015). The prospect here is to engineer a strongly correlated phase such as a heavy-fermion metal or a Kondo insulator.

Molecules and superconductivity: Toward Majorana modes.-When magnetic molecules weakly couple to superconductors, the remnant of the molecule's magnetic moment can be seen as a pair of in-gap resonances (Hatter et al., 2015; Island et al., 2017) termed Yu-Shiba-Rusinov states. The versatility and control allowed by the molecular design has facilitated the observation of a quantum phase transition (Farinacci et al., 2018). The intense activity in this field is motivated by theoretical predictions of Majorana modes in spin chains (Choy et al., 2011; Nadj-Perge et al., 2013; Pientka, Glazman, and von Oppen, 2013).

\section{Toward time-dependent studies: Molecular plasmonics}

The study of dynamical and light-induced phenomena in single-molecule transport is a long-standing challenge in the field. A short review and perspective was given by Thoss and Evers (2018).

Indeed, single-molecule transport under illumination has already been investigated for some time (Natelson, $\mathrm{Li}$, and Herzog, 2013). However, a breakthrough was reached recently by combining externally applied terahertz pulses with lowtemperature scanning tunneling microscopy (Cocker et al., 2016). In this way, time resolutions of $100 \mathrm{fs}$ were achieved with an eye toward achieving even higher resolutions.

\section{Toward devices: CISS and molecular-nuclear spintronics}

Many forms of switching in single-molecule junctions have been investigated, and a recent review was given by Ke et al. (2020). The magnetic degrees of freedom of molecular matter have been investigated in the fields of molecular magnets and molecular spintronics. Phenomena and activities in these fields are rich and deserve reviews of their own. We have only touched upon the chirality-induced spin selectivity; despite the effect not being well understood, applications are already under investigation, for instance, for separating enantiomers in chemical synthesis (BanerjeeGhosh et al., 2018).

We want to mention yet another development that is as speculative as it is stimulating: while the coherence times of electronic spins are believed to be too short to allow for quantum information processing, the lifetimes of nuclear spins located on isolated molecules are much longer. This observation has nourished hopes to use molecular-nuclear spins for applications in quantum computing (Moreno-Pineda et al., 2018). Indeed, the experimental control of nuclear spin states in isolated molecules has been demonstrated in a strong sequence of experiments (Thiele et al., 2014; Ganzhorn et al., 2016).

\section{ACKNOWLEDGMENTS}

Over the years we have profited from discussions with many scientists in the field, many more than we are able to list and acknowledge here. Specifically we want to express our gratitude to Alexey Bagrets, Richard Berndt, Marius Buerkle, Andrea Donarini, David Egger, Karin Fink, Thomas Frederiksen, Per Hedegård, Manabu Kiguchi, Denis Kochan, Leeor Kronik, Manohar Kumar, Stefan Kurth, Nicolás Lorente, Magdalena Marganska-Lyzniak, Marcel Mayor, Ron Naaman, Jeff Neaton, Abraham Nitzan, Tomáš Novotný, Jens Paaske, Fabian Pauly, Jascha Repp, Mario Ruben, Elke Scheer, Peter Schmitteckert, Gemma Solomon, Robert Stadler, Charles Stafford, Gianluca Stefanucci, Oren Tal, Jos Thijssen, Michael Thoss, Sense Jan van der Molen, Herre van der Zant, Héctor Vázquez, Latha Venkataraman, Felix von Oppen, Heiko Weber, Florian Weigend, Maarten Wegewijs, and Wulf Wulfhekel. We thank Nicolás Lorente, Jascha Repp, Michael Thoss, and, in particular, Mark Hybertsen for their careful reading of the manuscript and helpful feedback. This work was supported by the Netherlands Organisation for Scientific Research (NWO/OCW) as part of the Frontiers of Nanoscience program and program ENW.NVP.2018.001, by the Deutsche Forschungsgemeinschaft under Projects No. EV30/8-1 and No. EV30/111 and under SFB 1277 Projects No. B01 and No. A03, and by the PRIMUS/Sci/09 program of Charles University.

\section{REFERENCES}

Adak, O., R. Korytár, A. Joe, F. Evers, and L. Venkataraman, 2015, Nano Lett. 15, 3716.

Agraï, N., A. Levy Yeyati, and J. M. van Ruitenbeek, 2003, Phys. Rep. 377, 81.

Agraït, N., J. G. Rodrigo, and S. Vieira, 1993, Phys. Rev. B 47, 12345.

Agraït, N., C. Untiedt, G. Rubio-Bollinger, and S. Vieira, 2002, Phys. Rev. Lett. 88, 216803.

Akkerman, H. B., and B. de Boer, 2008, J. Phys. Condens. Matter 20, 013001.

Alexander, S., and P. W. Anderson, 1964, Phys. Rev. 133, A1594.

Anderson, P. W., 1961, Phys. Rev. 124, 41.

Appelbaum, J., 1966, Phys. Rev. Lett. 17, 91.

Appelbaum, J., 1967, Phys. Rev. 154, 633.

Aradhya, S. V., and L. Venkataraman, 2013, Nat. Nanotechnol. 8, 399. 
Armstrong, N., R. C. Hoft, A. McDonagh, M. B. Cortie, and M. J. Ford, 2007, Nano Lett. 7, 3018.

Arnold, A., F. Weigend, and F. Evers, 2007, J. Chem. Phys. 126, 174101.

Arroyo, C. R., E. Leary, A. Castellanos-Gómez, G. Rubio-Bollinger, M. T. González, and N. Agraït, 2011, J. Am. Chem. Soc. 133, 14313.

Arroyo, C. R., S. Tarkuc, R. Frisenda, J. S. Seldenthuis, C. H. Worde, R. Eelkema, F. C. Grozema, and H.S. J. van der Zant, 2013, Angew. Chem., Int. Ed. Engl. 52, 3152.

Aryasetiawan, F., and O. Gunnarsson, 1998, Rep. Prog. Phys. 61, 237.

Ateşçi, H., V. Kaliginedi, J. A. Celis Gil, H. Ozawa, J. M. Thijssen, P. Broekmann, M. Haga, and S. J. van der Molen, 2018, Nat. Nanotechnol. 13, 117.

Aulbur, W. G., L. Jönsson, and J.W. Wilkins, 1999, in Solid State Physics, edited by H. Ehrenreichand F. Spaepen (Academic, New York).

Aviram, A., and M. A. Ratner, 1974, Chem. Phys. Lett. 29, 277.

Avriller, R., and T. Frederiksen, 2012, Phys. Rev. B 86, 155411.

Avriller, R., and A. Levy Yeyati, 2009, Phys. Rev. B 80, 041309.

Baer, R., and D. Neuhauser, 2002, J. Am. Chem. Soc. 124, 4200.

Ballmann, S., R. Härtle, P. B. Coto, M. Elbing, M. 1 Mayor, M. R. Bryce, M. Thoss, and H. B. Weber, 2012, Phys. Rev. Lett. 109, 056801.

Banerjee-Ghosh, K., et al., 2018, Science 360, 1331.

Bardarson, J. H., 2008, J. Phys. A 41, 405203.

Bardeen, J., 1961, Phys. Rev. Lett. 6, 57.

Basch, H., R. Cohen, and M. A. Ratner, 2005, Nano Lett. 5, 1668.

Batra, A., P. Darancet, Q. Chen, J. S. Meisner, J. R. Widawsky, J. B. Neaton, C. Nuckolls, and L. Venkataraman, 2013, Nano Lett. 13, 6233.

Bechstedt, F., 2015, Many-Body Approach to Electronic Excitations: Concepts and Application (Springer-Verlag, Berlin).

Bedkihal, S., M. Bandyopadhyay, and D. Segal, 2013, Phys. Rev. B 87, 045418.

Bera, S., G. D. Tomasi, F. Weiner, and F. Evers, 2017, Phys. Rev. Lett. 118, 196801.

Bergfield, J. P., H. M. Heitzer, C. van Dyck, T. J. Marks, and M. A. Ratner, 2015, ACS Nano 9, 6412.

Bergfield, J. P., Z.-F. Liu, K. Burke, and C. A. Stafford, 2012, Phys. Rev. Lett. 108, 066801.

Bergfield, J. P., G. C. Solomon, C. A. Stafford, and M. A. Ratner, 2011, Nano Lett. 11, 2759.

Bickers, N. E., 1987, Rev. Mod. Phys. 59, 845.

Bode, N., S. V. Kusminskiy, R. Egger, and F. von Oppen, 2012, Beilstein J. Nanotechnol. 3, 144.

Bopp, J. M., S. Tewari, C. Sabater, and J. M. van Ruitenbeek, 2017, Low Temp. Phys. 43, 905.

Borda, L., G. Zaránd, W. Hofstetter, B. I. Halperin, and J. von Delft, 2003, Phys. Rev. Lett. 90, 026602.

Borges Pontes, R., A. R. Rocha, S. Sanvito, A. Fazzio, and A. J. Roque da Silva, 2011, ACS Nano 5, 795.

Brandbyge, M., N. Kobayashi, and M. Tsukada, 1999, Phys. Rev. B 60, 17064.

Brandbyge, M., J.-L. Mozos, P. Ordejón, J. Taylor, and K. Stokbro, 2002, Phys. Rev. B 65, 165401.

Brooke, C., A. Vezzoli, S. J. Higgins, L. A. Zotti, J. J. Palacios, and R. J. Nichols, 2015, Phys. Rev. B 91, 195438.

Bruot, C., J. Hihath, and N. Tao, 2012, Nat. Nanotechnol. 7, 35.

Bruus, H., and K. Flensberg, 2004, Many-Body Quantum Theory in Condensed Matter Physics: An Introduction (Oxford University Press, New York).
Bulla, R., T. A. Costi, and T. Pruschke, 2008, Rev. Mod. Phys. 80, 395.

Burke, K., M. Köntopp, and F. Evers, 2006, Phys. Rev. B 73, 121403(R).

Burzurí, E., Y. Yamamoto, M. Warnock, X. Zhong, K. Park, A. Cornia, and H. S. J. van der Zant, 2014, Nano Lett. 14, 3191.

Camarasa-Gómez, M., D. Hermangómez-Pérez, F. Evers, M. S. Inkpen, G. Lovat, and L. Venkataraman, 2019 (to be published), https://doi.org/10.26434/chemrxiv.12252059.v1.

Capozzi, B., J. Xia, O. Adak, E. J. Dell, Z.-F. Liu, J. C. Taylor, J. B. Neaton, L. M. Campos, and L. Venkataraman, 2015, Nat. Nanotechnol. 10, 522.

Cardamone, D. M., C. A. Stafford, and S. Mazumdar, 2006, Nano Lett. 6, 2422.

Caroli, C., R. Combescot, P. Nozières, and D. Saint-James, 1971, J. Phys. C 4, 916.

Celis Gil, J.A., and J.M. Thijssen, 2017, J. Chem. Phys. 147, 084102.

Chen, F., X. Li, J. Hihath, Z. Huang, and N. Tao, 2006, J. Am. Chem. Soc. 128, 15874.

Chen, W., J. R. Widawsky, H. Vázquez, S. T. Schneebeli, M. S. Hybertsen, R. Breslow, and L. Venkataraman, 2011, J. Am. Chem. Soc. 133, 17160.

Chen, X., M. Roemer, L. Yuan, W. Du, D. Thompson, E. del Barco, and C. A. Nijhuis, 2017, Nat. Nanotechnol. 12, 797.

Cheng, Z.-L., R. Skouta, H. Vázquez, J. R. Widawsky, S. Schneebeli, W. Chen, M. S. Hybertsen, R. Breslow, and L. Venkataraman, 2011, Nat. Nanotechnol. 6, 353.

Choi, M.-S., R. López, and R. Aguado, 2005, Phys. Rev. Lett. 95, 067204.

Choi, S. H., B. Kim, and C. D. Frisbie, 2008, Science 320, 1482.

Choi, S. H., C. Risko, M. Ruiz Delgado, B. Kim, J.-L. Brédas, and C. D. Frisbie, 2010, J. Am. Chem. Soc. 132, 4358.

Choy, T.-P., J. M. Edge, A. Akhmerov, and C. W. J. Beenakker, 2011, Phys. Rev. B 84, 195442.

Cocker, T. L., D. Peller, P. Yu, J. Repp, and R. Huber, 2016, Nature (London) 539, 263.

Coleman, P., 2015, Introduction to Many-Body Physics (Cambridge University Press, Cambridge, England).

Costi, T. A., A. C. Hewson, and V. Zlatić, 1994, J. Phys. Condens. Matter 6, 2519.

Craven, G. T., and A. Nitzan, 2017a, J. Chem. Phys. 146, 092305.

Craven, G. T., and A. Nitzan, 2017b, Phys. Rev. Lett. 118, 207201.

Cuevas, J. C., J. Heurich, F. Pauly, W. Wenzel, and G. Schön, 2003, Nanotechnology 14, R29.

Cui, L., W. Jeong, S. Hur, M. Matt, J. C. Klöckner, F. Pauly, P. Nielaba, J. C. Cuevas, E. Meyhofer, and P. Reddy, 2017, Science 355, 1192.

Cui, L., R. Miao, C. Jiang, E. Meyhofer, and P. Reddy, 2017, J. Chem. Phys. 146, 092201.

Cui, X. D., A. Primak, X. Zarate, J. Tomfohr, O. F. Sankey, A. L. Moore, T. A. Moore, D. Gust, G. Harris, and S. M. Lindsay, 2001, Science 294, 571.

Cui, X. D., A. Primak, X. Zarate, J. Tomfohr, O. F. Sankey, A. L. Moore, T. A. Moore, D. Gust, L. A. Nagahara, and S. M. Lindsay, 2002, J. Phys. Chem. B 106, 8609.

Dalum, S., and P. Hedegård, 2019, Nano Lett. 19, 5253.

Darwish, N., I. Díez-Pérez, P. Da Silva, N. Tao, J. J. Gooding, and M. N. Paddon-Row, 2012, Angew. Chem., Int. Ed. Engl. 51, 3203. Dasgupta, A., S. Bera, F. Evers, and M. J. van Setten, 2012, Phys. Rev. B 85, 125433.

de Bruijckere, J., P. Gehring, M. Palacios-Corella, M. ClementeLeón, E. Coronado, J. Paaske, P. Hedegård, and H. S. van der Zant, 2019, Phys. Rev. Lett. 122, 197701. 
Delle Site, L., 2018, Adv. Theory Simul. 1, 1800056.

DiLullo, A., S.-H. Chang, N. Baadji, K. Clark, J.-P. Klöckner, M.-H. Prosenc, S. Sanvito, R. Wiesendanger, G. Hoffmann, and S.-W. Hla, 2012, Nano Lett. 12, 3174.

Di Ventra, M., 2008, Electrical Transport in Nanoscale Systems (Cambridge University Press, Cambridge, England).

Di Ventra, M., S. Pantelides, and N. Lang, 2000, Phys. Rev. Lett. 84, 979.

Di Ventra, M., S. T. Pantelides, and N. D. Lang, 2002, Phys. Rev. Lett. 88, 046801.

Djukic, D., K. S. Thygesen, C. Untiedt, R. H. M. Smit, K. W. Jacobsen, and J. M. van Ruitenbeek, 2005, Phys. Rev. B 71, 161402.

Djukic, D., and J. M. van Ruitenbeek, 2006, Nano Lett. 6, 789.

Donarini, A., G. Begemann, and M. Grifoni, 2010, Phys. Rev. B 82, 125451.

Doppagne, B., M. C. Chong, H. Bulou, A. Boeglin, F. Scheurer, and G. Schull, 2018, Science 361, 251.

Droghetti, A., and I. Rungger, 2017, Phys. Rev. B 95, 085131.

Dulić, D., S. van der Molen, T. Kudernac, H. Jonkman, J. J. D. de Jong, T. N. Bowden, J. van Esch, B. L. Feringa, and B. J. van Wees, 2003, Phys. Rev. Lett. 91, 207402.

Dundas, D., E. J. McEniry, and T. N. Todorov, 2009, Nat. Nanotechnol. 4, 99.

Egger, R., and A. O. Gogolin, 2008, Phys. Rev. B 77, 113405.

Elbing, M., R. Ochs, M. Koentopp, M. Fischer, C. von Hänisch, F. Weigend, F. Evers, H. B. Weber, and M. Mayor, 2005, Proc. Natl. Acad. Sci. U.S.A. 102, 8815.

Emberly, E., and G. Kirczenow, 2001, Phys. Rev. B 64, 235412.

Emberly, E. G., and G. Kirczenow, 1998, Phys. Rev. B 58, 10911.

Enkovaara, J., et al., 2010, J. Phys. Condens. Matter 22, 253202.

Entin-Wohlman, O., Y. Imry, and A. Aharony, 2009, Phys. Rev. B 80, 035417.

Eremko, A. A., and V. M. Loktev, 2013, Phys. Rev. B 88, 165409.

Erpenbeck, A., C. Schinabeck, U. Peskin, and M. Thoss, 2018, Phys. Rev. B 97, 235452.

Esat, T., B. Lechtenberg, T. Deilmann, C. Wagner, P. Krüger, R. Temirov, M. Rohlfing, F. B. Anders, and F. S. Tautz, 2016, Nat. Phys. 12, 867.

Evers, F., and A. Arnold, 2011, Lect. Notes Phys. 820, 27.

Evers, F., and K. Burke, 2007, in Nano and Molecular Electronics Handbook, edited by S. Lyshevski (CRC Press, New York), Chap. 24.

Evers, F., and P. Schmitteckert, 2013, Phys. Status Solidi B 250, 2330.

Evers, F., F. Weigend, and M. Koentopp, 2004, Phys. Rev. B 69, 235411.

Faber, C., P. Boulanger, C. Attaccalite, I. Duchemin, and X. Blase, 2014, Phil. Trans. R. Soc. A 372, 20130271.

Fajtlowicz, S., P. E. John, and H. Sachs, 2005, Croat. Chem. Acta 78, 195.

Faleev, S. V., F. Leonard, D. A. Stewart, and M. van Schilfgaarde, 2005, Phys. Rev. B 71, 195422.

Fano, U., 1961, Phys. Rev. 124, 1866.

Farid, B., 1999, Electron Correlation in the Solid State, Cambridge Studies in Magnetism (World Scientific, Singapore).

Farinacci, L., G. Ahmadi, G. Reecht, M. Ruby, N. Bogdanoff, O. Peters, B. W. Heinrich, F. von Oppen, and K. J. Franke, 2018, Phys. Rev. Lett. 121, 196803.

Fatayer, S., B. Schuler, W. Steurer, I. Scivetti, J. Repp, L. Gross, M. Persson, and G. Meyer, 2018, Nat. Nanotechnol. 13, 376.

Fernández, J., A. A. Aligia, and A. M. Lobos, 2015, Europhys. Lett. 109, 37011
Ferrer, J., C. Lambert, V. García-Suárez, D. Z. Manrique, D. Visontai, L. Oroszlany, R. Rodríguez-Ferradás, I. Grace, S. Bailey, and K. Gillemot, 2014, New J. Phys. 16, 093029.

Fiolhais, C., F. Nogueira, and M. E. Marques, 2003, A Primer in Density Functional Theory (Springer-Verlag, Berlin).

Florens, S., A. Freyn, N. Roch, W. Wernsdorfer, F. Balestro, P. Roura-Bas, and A. A. Aligia, 2011, J. Phys. Condens. Matter 23, 243202.

Fock, J., M. Leijnse, K. Jennum, A. S. Zyazin, J. Paaske, P. Hedegård, M. B. Nielsen, and H. S. J. Van der Zant, 2012, Phys. Rev. B 86, 235403.

Fowler, P. W., B. T. Pickup, and T. Z. Todorova, 2011, Pure Appl. Chem. 83, 1515.

Fowler, P. W., B. T. Pickup, T. Z. Todorova, and W. Myrvold, 2009, J. Chem. Phys. 131, 044104.

Franke, K. J., G. Schulze, and J. I. Pascual, 2011, Science 332, 940.

French, W. R., C. R. Iacovella, I. Rungger, A. M. Souza, S. Sanvito, and P. T. Cummings, 2013, Nanoscale 5, 3654.

Frisenda, R., V. A. E. C. Janssen, F. C. Grozema, H. S. J. van der Zant, and N. Renaud, 2016, Nat. Chem. 8, 1099.

Frisenda, R., R. Gaudenzi, C. Franco, M. Mas-Torrent, C. Rovira, J. Veciana, I. Alcon, S. T. Bromley, E. Burzurí, and H. S. van der Zant, 2015, Nano Lett. 15, 3109.

Frisenda, R., M. L. Perrin, H. Valkenier, J. C. Hummelen, and H. S. J. van der Zant, 2013, Phys. Status Solidi B 250, 2431.

Frisenda, R., and H. S. J. van der Zant, 2016, Phys. Rev. Lett. 117, 126804

Fu, Y.-S., Q.-K. Xue, and R. Wiesendanger, 2012, Phys. Rev. Lett. 108, 087203 .

Fujii, S., U. Akiba, and M. Fujihira, 2008, Chem. Lett. 37, 408.

Gagliardi, A., G. C. Solomon, A. Pecchia, T. Frauenheim, A. Di Carlo, N. S. Hush, and J. R. Reimers, 2007, Phys. Rev. B 75, 174306.

Galperin, M., M. A. Ratner, and A. Nitzan, 2005, Nano Lett. 5, 125.

Ganzhorn, M., S. Klyatskaya, M. Ruben, and W. Wernsdorfer, 2016, Nat. Commun. 7, 11443.

Gao, B., E. A. Osorio, K. B. Gaven, and H. S. J. van der Zant, 2009, Nanotechnology 20, 415207.

García, R., et al., 2015, Beilstein J. Org. Chem. 11, 1068.

García, Y., J. J. Palacios, E. SanFabián, J. A. Vergés, A. J. PérezJiménez, and E. Louis, 2004, Phys. Rev. B 69, 041402.

García-Suárez, V. M., T. Kostyrko, S. Bailey, C. Lambert, and B. R. Bułka, 2007, Phys. Status Solidi B 244, 2443.

García-Suárez, V. M., A. R. Rocha, S. W. Bailey, C. J. Lambert, S. Sanvito, and J. Ferrer, 2005, Phys. Rev. B 72, 045437.

Garner, M. H., et al., 2018, Nature (London) 558, 415.

Garrigues, A. R., L. Yuan, L. Wang, E. R. Mucciolo, D. Thompon, E. del Barco, and C. A. Nijhuis, 2016, Sci. Rep. 6, 26517.

Gaudenzi, R., M. Misiorny, E. Burzurí, M. R. Wegewijs, and H. S. van der Zant, 2017, J. Chem. Phys. 146, 092330.

Geddes, N. J., J. R. Sambles, D. J. Davis, W. G. Parker, and D. J. Sandman, 1990, Appl. Phys. Lett. 56, 1916.

Geng, Y., S. Sangtarash, C. Huang, H. Sadegh, Y. Fu, W. Hong, T. Wandlowski, S. Decurtins, C. J. Lambert, and S.-X. Liu, 2015, J. Am. Chem. Soc. 137, 4469.

Géranton, G., C. Seiler, A. Bagrets, L. Venkataraman, and F. Evers, 2013, J. Chem. Phys. 139, 234701.

Gerstin, J., K. Kaasbjerg, and A. Nitzan, 2013, J. Chem. Phys. 139, 114111.

Gil, M., T. Malinowski, M. Iazykov, and H. R. Klein, 2018, J. Appl. Phys. 123, 104303.

Glazman, L. I., and M. E. Raikh, 1988, JETP Lett. 47, 452. 
Göhler, B., V. Hamelbeck, T. Z. Markus, M. Kettner, G. F. Hanne, Z. Vager, R. Naaman, and H. Zacharias, 2011, Science 331, 894.

Goldhaber-Gordon, D., J. Göres, M. A. Kastner, H. Shtrikman, D. Mahalu, and U. Meirav, 1998, Phys. Rev. Lett. 81, 5225.

González, M. T., S. Wu, R. Huber, S. J. van der Molen, C. Schönenberger, and M. Calame, 2006, Nano Lett. 6, 2238.

Greuling, A., M. Rohlfing, R. Temirov, F. S. Tautz, and F. B. Anders, 2011, Phys. Rev. B 84, 125413.

Grigoriev, A., J. Sköldberg, G. Wendin, and Ž. Crljen, 2006, Phys. Rev. B 74, 045401.

Gröning, O., et al., 2018, Nature (London) 560, 209.

Groth, C. W., M. Wimer, A. R. Akhmerov, and X. Waintal, 2014, New J. Phys. 16, 063065.

Grunder, S., R. Huber, V. Horhoiu, M. T. González, C. Schönenberger, M. Calame, and M. Mayor, 2007, J. Org. Chem. 72, 8337.

Guédon, C. M., H. Valkenier, T. Markussen, K. S. Thygesen, J. C. Hummelen, and S. J. van der Molen, 2012, Nat. Nanotechnol. 7, 305 .

Gunasekaran, S., D. Hernangomez-Perez, J. Davydenko, S. Marder, F. Evers, and L. Venkataraman, 2018, Nano Lett. 18, 6387.

Guo, A.-M., E. Diaz, C. Gaul, R. Gutierrez, F. Dominguez-Adame, G. Cuniberti, and Q.-F. Sun, 2014, Phys. Rev. B 89, 205434.

Guo, A.-M., and Q.-F. Sun, 2012a, Phys. Rev. B 86, 115441.

Guo, A.-M., and Q.-F. Sun, 2012b, Phys. Rev. Lett. 108, 218102.

Guo, A.-M., and Q.-F. Sun, 2014, Proc. Natl. Acad. Sci. U.S.A. 111, 11658.

Gutierrez, R., E. Diaz, R. Naaman, and G. Cuniberti, 2012, Phys. Rev. B 85, 081404.

Hackenbroich, G., 2001, Phys. Rep. 343, 463.

Häfner, V., J. Schindler, N. Weik, T. Mayer, S. Balakrischnan, R. Narayanan, S. Bera, and F. Evers, 2014, Phys. Rev. Lett. 113, 186802.

Haiss, W., S. Martin, L. E. Scullion, L. Bouffier, S. J. Higgins, and R. J. Nichols, 2009, Phys. Chem. Chem. Phys. 11, 10831.

Haiss, W., R. J. Nichols, H. van Zalinge, S. J. Higgins, D. Bethell, and D. J. Schiffrin, 2004, Phys. Chem. Chem. Phys. 6, 4330.

Haiss, W., H. van Zalinge, S. J. Higgins, D. Bethell, H. Höbenreich, D. J. Schiffrin, and R. J. Nichols, 2003, J. Am. Chem. Soc. 125, 15294.

Haiss, W., et al., 2008, J. Phys. Condens. Matter 20, 374119.

Häkkinen, H., 2012, Nat. Chem. 4, 443.

Hansen, T., G. C. Solomon, D. Q. Andrews, and M. A. Ratner, 2009, J. Chem. Phys. 131, 194704.

Harary, F., 1962, SIAM Rev. 4, 202.

Härtle, R., M. Butzin, O. Rubio-Pons, and M. Thoss, 2011, Phys. Rev. Lett. 107, 046802.

Hatter, N., B. W. Heinrich, M. Ruby, J. I. Pascual, and K. J. Franke, 2015, Nat. Commun. 6, 8988.

Haug, H. J. W., and A.-P. Jauho, 2008, Quantum Kinetics in Transport and Optics of Semiconductors, 2nd ed., Vol. 2 (SpringerVerlag, Berlin).

Haupt, F., T. Novotný, and W. Belzig, 2009, Phys. Rev. Lett. 103, 136601.

Haupt, F., T. Novotný, and W. Belzig, 2010, Phys. Rev. B 82, 165441

He, J., O. Sankey, M. Lee, N. Tao, X. Li, and S. Lindsay, 2006, Faraday Discuss. 131, 145.

Hedin, L., 1999, J. Phys. Condens. Matter 11, R489.

Heeger, A., S. Kivelson, J. R. Schrieffer, and W.-P. Su, 1988, Rev. Mod. Phys. 60, 781.

Hewson, A. C., 1993, The Kondo Problem to Heavy Fermions, Cambridge Studies in Magnetism (Cambridge University Press, Cambridge, England).
Hihath, J., C. R. Arroyo, G. Rubio-Bollinger, N. Tao, and N. Agraï, 2008, Nano Lett. 8, 1673.

Hines, T., I. Diez-Perez, J. Hihath, H. Liu, Z.-S. Wang, J. Zhao, G. Zhou, K. Müllen, and N. Tao, 2010, J. Am. Chem. Soc. 132, 11658.

Hirjibehedin, C. F., C.-Y. Lin, A. F. Otte, M. Ternes, C. P. Lutz, B. A. Jones, and A. J. Heinrich, 2007, Science 317, 1199.

Ho, P. S., and T. Kwok, 1989, Rep. Prog. Phys. 52, 301.

Hofstetter, W., and H. Schoeller, 2001, Phys. Rev. Lett. 88, 016803.

Holzer, C., and W. Klopper, 2017, J. Chem. Phys. 147, 181101.

Hong, W., H. Li, S.-X. Liu, Y. Fu, J. Li, V. Kaliginedi, S. Decurtins, and T. Wandlowski, 2012, J. Am. Chem. Soc. 134, 19425.

Houck, A. A., J. Labaziewicz, E. K. Chan, J. A. Folk, and I. L. Chuang, 2005, Nano Lett. 5, 1685.

Huang, B., et al., 2018, J. Am. Chem. Soc. 140, 17685.

Huang, Z., F. Chen, R. D'agosta, P. A. Bennett, M. Di Ventra, and N. Tao, 2007, Nat. Nanotechnol. 2, 698.

Huber, R., et al., 2008, J. Am. Chem. Soc. 130, 1080.

Huisman, E. H., M. L. Trouwborst, F. L. Bakker, B. de Boer, B. J. van Wees, and S. J. van der Molen, 2008, Nano Lett. 8, 3381.

Huntington, H. B., and A. R. Grone, 1961, J. Phys. Chem. Solids 20, 76.

Hüser, F., and G. S. Solomon, 2015, J. Phys. Chem. C 119, 14056. Hybertsen, M. S., and L. Venkataraman, 2016, Acc. Chem. Res. 49, 452.

Hybertsen, M. S., L. Venkataraman, J. E. Klare, A. C. Whalley, M. L. Steigerwald, and C. Nuckolls, 2008, J. Phys. Condens. Matter 20, 374115.

Imry, Y., 2002, Introduction to Mesoscopic Physics (Oxford University Press, New York).

Inkpen, M. S., Z.-F. Liu, H. Li, L. M. Campos, J. B. Neaton, and L. Venkataraman, 2018, preprint.

Island, J. O., et al., 2017, Phys. Rev. Lett. 118, 117001.

Iwane, M., S. Fujii, and M. Kiguchi, 2017, Sensors 17, 1901.

Jacob, D., 2015, J. Phys. Condens. Matter 27, 245606.

Jacob, D., and J. J. Palacios, 2011, J. Chem. Phys. 134, 044118.

Jacobson, P., T. Herden, M. Muenks, G. Laskin, O. Brovko, V. Stepanyuk, M. Ternes, and K. Kern, 2015, Nat. Commun. 6, 8536.

Jang, S.-Y., P. Reddy, A. Majumdar, and R. A. Segalman, 2006, Nano Lett. 6, 2362.

Jarillo-Herrero, P., J. Kong, H. S. J. van der Zant, C. Dekker, L. P. Kouwenhoven, and S. De Franceschi, 2005, Nature (London) 434, 484.

Jasper-Tönnies, T., A. Garcia-Lekue, T. Frederiksen, S. Ulrich, R. Herges, and R. Berndt, 2017, Phys. Rev. Lett. 119, 066801.

Jayaprakash, C., H. R. Krishna-murthy, and J. W. Wilkins, 1981, Phys. Rev. Lett. 47, 737.

Jayaprakash, C., H. R. Krishna-murthy, and J. W. Wilkins, 1982, J. Appl. Phys. 53, 2142.

Jeong, H., D. Kim, D. Xiang, and T. Lee, 2017, ACS Nano 11, 6511. Jia, C., and X. Guo, 2013, Chem. Soc. Rev. 42, 5642.

Jin, C., and K. S. Thygesen, 2014, Phys. Rev. B 89, 041102(R).

Joachim, C., J. K. Gimzewski, R. R. Schlittler, and C. Chavy, 1995, Phys. Rev. Lett. 74, 2102.

Joachim, C., and M. Magoga, 2002, Chem. Phys. 281, 347.

Jones, D., and A. Troisi, 2007, J. Phys. Chem. C 111, 14567.

Kaasbjerg, K., and K. Flensberg, 2011, Phys. Rev. B 84, 115457.

Kaasbjerg, K., T. Novotný, and A. Nitzan, 2013, Phys. Rev. B 88, 201405(R).

Kaat, G. A., and K. Flensberg, 2005, Phys. Rev. B 71, 155408.

Kaliginedi, V., P. Moreno-García, H. Valkenier, W. Hong, V. M. García-Suárez, P. Buiter, J. L. H. Otten, J. C. Hummelen, C. J. Lambert, and T. Wandlowski, 2012, J. Am. Chem. Soc. 134, 5262. 
Kaneko, S., C. Motta, G. P. Brivio, and M. Kiguchi, 2013, Nanotechnology 24, 315201.

Kaneko, S., et al., 2019, Chem. Sci. 10, 6261.

Karan, S., C. García, M. Karolak, D. Jacob, N. Lorente, and R. Berndt, 2018, Nano Lett. 18, 88.

Karimi, M., S. G. Bahoosh, M. Herz, R. Hayakawa, F. Pauly, and E. Scheer, 2016, Nano Lett. 16, 1803.

Ke, G., C. Duan, F. Huang, and X. Guo, 2020, InfoMat 2, 92.

Ke, S.-H., H. U. Baranger, and W. Yang, 2005, J. Chem. Phys. 123, 114701.

Ke, S.-H., H. U. Baranger, and W. Yang, 2007, J. Chem. Phys. 127, 144107.

Ke, S.-H., W. Yang, and H. U. Baranger, 2008, Nano Lett. 8, 3257.

Kershaw, V. F., and D. S. Kosov, 2017, J. Chem. Phys. 147, 224109.

Kiguchi, M., N. Sekiguchi, and K. Murakoshi, 2008, J. Phys. Conf. Ser. 100, 052059.

Kiguchi, M., O. Tal, S. Wohlthat, F. Pauly, M. Krieger, D. Djukic, J. C. Cuevas, and J. M. van Ruitenbeek, 2008, Phys. Rev. Lett. 101, 046801.

Kim, S., and Y.-W. Son, 2013, Phys. Rev. B 87, 195423.

Kim, Y., T. J. Hellmuth, M. Bürkle, F. Pauly, and E. Scheer, 2011, ACS Nano 5, 4104.

Kim, Y., T. Pietsch, A. Erbe, W. Belzig, and E. Scheer, 2011, Nano Lett. 11, 3734.

Kim, Y., H. Song, F. Strigl, H.-F. Pernau, T. Lee, and E. Scheer, 2011, Phys. Rev. Lett. 106, 196804.

Kiselev, A. A., and K. W. Kim, 2005, Phys. Rev. B 71, 153315.

Koch, J., and F. von Oppen, 2005, Phys. Rev. Lett. 94, 206804.

Koch, J., F. von Oppen, and A. V. Andreev, 2006, Phys. Rev. B 74, 205438.

Koch, M., F. Ample, C. Joachim, and L. Grill, 2012, Nat. Nanotechnol. 7, 713.

Kondo, H., H. Kino, J. Nara, T. Ozaki, and T. Ohno, 2006, Phys. Rev. B 73, 235323.

Kondo, J., 1964, Prog. Theor. Phys. 32, 37.

Könemann, J., B. Kubala, J. König, and R. J. Haug, 2006, Phys. Rev. B 73, 033313.

Korytár, R., and N. Lorente, 2011, J. Phys. Condens. Matter 23, 355009.

Korytár, R., N. Lorente, and J.-P. Gauyacq, 2012, Phys. Rev. B 85, 125434.

Korytár, R., D. Xenioti, P. Schmitteckert, M. Alouani, and F. Evers, 2014, Nat. Commun. 5, 5000.

Kouwenhoven, L., and L. Glazman, 2001, Phys. World 14, 33.

Krane, N., C. Lotze, G. Reecht, L. Zhang, A. L. Briseno, and K. J. Franke, 2018, ACS Nano 12, 11698.

Krans, J. M., C. J. Muller, I. K. Yanson, T. C. M. Govaert, R. Hesper, and J. M. van Ruitenbeek, 1993, Phys. Rev. B 48, 14721.

Kristensen, I. S., M. Paulsson, K. S. Thygesen, and K. W. Jacobsen, 2009, Phys. Rev. B 79, 235411.

Krstić, P., X.-G. Zhang, and W. Buttler, 2002, Phys. Rev. B 66, 205319.

Kubatkin, S., A. Danilov, M. Hjort, J. Cornil, J.-L. Brédas, N. Stuhr-Hansen, P. Hedegård, and T. Bjørnholm, 2003, Nature (London) 425, 698.

Kügel, J., P.-J. Hsu, M. Böhme, K. Schneider, J. Senkpiel, D. Serrate, M. Bode, and N. Lorente, 2018, Phys. Rev. Lett. 121, 226402.

Kumar, M., R. Avriller, A. L. Yeyati, and J. M. van Ruitenbeek, 2012, Phys. Rev. Lett. 108, 146602.

Lafferentz, L., F. Ample, H. Yu, S. Hecht, C. Joachim, and L. Grill, 2009, Science 323, 1193.

Lambert, C. J., 2015, Chem. Soc. Rev. 44, 875.
Langlais, V. L., R. R. Schlittler, H. Tang, A. Gourdon, C. Joachim, and J. K. Gimzewski, 1999, Phys. Rev. Lett. 83, 2809.

Larsen, A., et al., 2017, J. Phys. Condens. Matter 29, 273002.

Lau, C. S., H. Sadeghi, G. Rogers, S. Sangtarash, P. Dallas, K. Porfyrakis, J. Warner, C. J. Lambert, G. A. D. Briggs, and J. A. Mol, 2016, Nano Lett. 16, 170.

Le Hur, K., and P. Simon, 2003, Phys. Rev. B 67, 201308.

Lee, M., M.-S. Choi, R. López, R. Aguado, J. Martinek, and R. Žitko, 2010, Phys. Rev. B 81, 121311.

Lee, W., K. Kim, W. Jeong, L. Zotti, F. Pauly, J. Cuevas, and P. Reddy, 2013, Nature (London) 498, 209.

Leijnse, M., and M. R. Wegewijs, 2008, Phys. Rev. B 78, 235424.

Leturcq, R., C. Stampfer, K. Inderbitzin, L. Durrer, C. Hierold, E. Mariani, M. G. Schultz, F. von Oppen, and K. Ensslin, 2009, Nat. Phys. 5, 327.

Li, C., I. Pobelov, T. Wandlowski, A. Bagrets, A. Arnold, and F. Evers, 2008, J. Am. Chem. Soc. 130, 318.

Li, X., J. He, J. Hihath, B. Xu, S. M. Lindsay, and N. Tao, 2006, J. Am. Chem. Soc. 128, 2135.

Li, Y., et al., 2018, arXiv:1806.00124.

Li, Z., and D. S. Kosov, 2007, Phys. Rev. B 76, 035415.

Liang, W., M. P. Shores, M. Bockrath, J. R. Long, and H. Park, 2002, Nature (London) 417, 725.

Lim, J. S., M.-S. Choi, M. Choi, R. López, and R. Aguado, 2006, Phys. Rev. B 74, 205119.

Liu, K., G. Li, X. Wang, and F. Wang, 2008, J. Phys. Chem. C 112, 4342.

Liu, R., S.-H. Ke, W. Yang, and H. U. Baranger, 2006, J. Chem. Phys. 124, 024718.

Liu, Z.-F., D. Egger, S. Refaely-Abramson, L. Kronik, and J. Neaton, 2017, J. Chem. Phys. 146, 092326.

Lobos, A. M., M. Romero, and A. A. Aligia, 2014, Phys. Rev. B 89, 121406.

Logan, D. E., C. J. Wright, and M. R. Galpin, 2009, Phys. Rev. B 80, 125117.

Longuet-Higgins, H. C., 1950, J. Chem. Phys. 18, 265.

Lörtscher, E., J. W. Ciszek, J. Tour, and H. Riel, 2006, Small 2, 973.

Lörtscher, E., H. B. Weber, and H. Riel, 2007, Phys. Rev. Lett. 98, 176807.

Lovey, D. A., and R. H. Romero, 2012, Chem. Phys. Lett. 530, 86.

Lu, Q., K. Liu, H. Zhang, Z. Du, X. Wang, and F. Wang, 2009, ACS Nano 3, 3861.

Lü, J.-T., M. Brandbyge, and P. Hedegøard, 2010, Nano Lett. 10, 1657.

Lü, J.-T., R. B. Christensen, J.-S. Wang, P. Hedegård, and M. Brandbyge, 2015, Phys. Rev. Lett. 114, 096801.

Lü, J.-T., B.-Z. Hu, P. Hedegård, and M. Brandbyge, 2019, Prog. Surf. Sci. 94, 21.

Ludoph, B., M. H. Devoret, D. Esteve, C. Urbina, and J. M. van Ruitenbeek, 1999, Phys. Rev. Lett. 82, 1530.

Ludoph, B., and J. M. van Ruitenbeek, 1999, Phys. Rev. B 59, 12290.

Lykkebo, J., A. Gagliardi, A. Pecchia, and G. C. Solomon, 2013, ACS Nano 7, 9183.

Lykkebo, J., A. Gagliardi, A. Pecchia, and G. C. Solomon, 2014, J. Chem. Phys. 141, 124119.

Maggio, E., G. C. Solomon, and A. Troisi, 2014, ACS Nano 8, 409.

Makarovski, A., A. Zhukov, J. Liu, and G. Finkelstein, 2007, Phys. Rev. B 75, 241407.

Malen, J. A., P. Doak, K. Baheti, T. D. Tilley, R. A. Segalman, and A. Majumdar, 2009, Nano Lett. 9, 1164.

Manrique, D. Z., C. Huang, M. Baghernejad, X. Zha, and O. A. AlOwaedi, 2015, Nat. Commun. 6, 6389. 
Markussen, T., J. Schiötz, and K. S. Thygesen, 2010, J. Chem. Phys. 132, 224104.

Markussen, T., R. Stadler, and K. S. Thygesen, 2010, Nano Lett. 10, 4260.

Markussen, T., R. Stadler, and K. S. Thygesen, 2011, Phys. Chem. Chem. Phys. 13, 14311.

Markussen, T., and K. S. Thygesen, 2014, Phys. Rev. B 89, 085420.

Marquardt, C., S. Grunder, A. Blaszczyk, S. Dehm, F. Hennrich, H. von Löhneysen, M. Mayor, and R. Krupke, 2010, Nat. Nanotechnol. 5, 863.

Martin, C. A., 2010, Ph.D. thesis (Casimir Research School).

Martin, C. A., D. Ding, and J. K. Sørensen, T. Bjørnholm, J. M. van Ruitenbeek, and H. S. J. van der Zant, 2008, J. Am. Chem. Soc. 130, 13198.

Martin, C. A., D. Ding, H.S. J. van der Zant, and J.M. van Ruitenbeek, 2008, New J. Phys. 10, 065008.

Matityahu, S., A. Aharony, O. Entin-Wohlman, and C. A. Balseiro, 2017, Phys. Rev. B 95, 085411.

Matityahu, S., Y. Utsumi, A. Aharony, O. Entin-Wohlman, and C. A. Balseiro, 2016, Phys. Rev. B 93, 075407.

Maul, R., and W. Wenzel, 2009, Phys. Rev. B 80, 045424.

Mayor, M., H. Weber, J. Reichert, M. Elbing, v. C. Hänisch, D. Beckmann, and M. Fischer, 2003, Angew. Chem., Int. Ed. Engl. 42, 5834.

Mayou, D., Y. Zhou, and M. Ernzerhof, 2013, J. Phys. Chem. C 117, 7870.

Medina, E., F. Lopez, M. A. Ratner, and V. Mujica, 2012, Europhys. Lett. 99, 17006.

Meir, Y., and N. S. Wingreen, 1992, Phys. Rev. Lett. 68, 2512.

Metzger, R. M., et al., 2003, J. Phys. Chem. B 107, 1021.

Meyer, J., V. Falko, and B. Altshuler, 2002, in NATO Science Series II: Strongly Correlated Fermions and Bosons in Low-Dimensional Disordered Systems, Vol. 72, edited by L. Lerner, B. Althsuler, V. Falko, and T. Giamarchi (Springer, Dordrecht), pp. 117-164.

Minamitani, E., N. Tsukahara, D. Matsunaka, Y. Kim, N. Takagi, and M. Kawai, 2012, Phys. Rev. Lett. 109, 086602.

Mishchenko, A., et al., 2010, Nano Lett. 10, 156.

Mitchell, A. K., K. G. L. Pedersen, P. Hedegård, and J. Paaske, 2017, Nat. Commun. 8, 15210.

Miyamachi, T., et al., 2012, Nat. Commun. 3, 938.

Moreland, J., S. Alexander, M. Cox, R. Sonnenfeld, and P. K. Hansma, 1983, Appl. Phys. Lett. 43, 387.

Moreno-Pineda, E., C. Godfrin, F. Balestro, W. Wernsdorfer, and M. Ruben, 2018, Chem. Soc. Rev. 47, 501.

Mowbray, D. J., G. Jones, and K. S. Thygesen, 2008, J. Chem. Phys. 128, 111103.

Mugarza, A., C. Krull, R. Robles, S. Stepanow, G. Ceballos, and P. Gambardella, 2011, Nat. Commun. 2, 490.

Mugarza, A., R. Robles, C. Krull, R. Korytár, N. Lorente, and P. Gambardella, 2012, Phys. Rev. B 85, 155437.

Muller, C. J., J. M. van Ruitenbeek, and L. J. de Jongh, 1992, Physica (Amsterdam) 191C, 485.

Naaman, R., and D. H. Waldeck, 2015, Annu. Rev. Chem. Biomol. Eng. 66, 263.

Nadj-Perge, S., I. K. Drozdov, B. A. Bernevig, and A. Yazdani, 2013, Phys. Rev. B 88, 020407.

Nandkishore, R., and D. A. Huse, 2015, Annu. Rev. Condens. Matter Phys. 6, 15.

Natelson, D., Y. Li, and J. B. Herzog, 2013, Phys. Chem. Chem. Phys. 15, 5262.

Neaton, J., M. S. Hybertsen, and S. G. Louie, 2006, Phys. Rev. Lett. 97, 216405
Néel, N., J. Kröger, L. Limot, T. Frederiksen, M. Brandbyge, and R. Berndt, 2007, Phys. Rev. Lett. 98, 065502.

Ng, M.-K., D.-C. Lee, and L. Yu, 2002, J. Am. Chem. Soc. 124, 11862.

Niklas, M., A. Trottmann, A. Donarini, and M. Grifoni, 2017, Phys. Rev. B 95, 115133.

Nishikawa, A., J. Tobita, Y. Kato, S. Fujii, M. Suzuki, and M. Fujihira, 2007, Nanotechnology 18, 424005.

Nitzan, A., 2001, Annu. Rev. Phys. Chem. 52, 681.

Nitzan, A., 2006, Chemical Dynamics in Condensed Phases, Oxford Graduate Texts (Oxford University Press, New York).

Novotný, T., F. Haupt, and W. Belzig, 2011, Phys. Rev. B 84, 113107

Nozières, P., 1974, J. Low Temp. Phys. 17, 31.

Nozières, P., and A. Blandin, 1980, J. Phys. (Paris) 41, 193.

Nuss, M., W. von der Linden, and E. Arrigoni, 2014, Phys. Rev. B 89, 155139

Obersteiner, V., G. Huhs, N. Papior, and E. Zojer, 2017, Nano Lett. 17, 7350 .

Okuyama, H., H. So, S. Hatta, T. Frederiksen, and T. Aruga, 2018, Surf. Sci. 678, 169.

Olavarria-Contreras, I. J., M. L. Perrin, Z. Chen, S. Klyatskaya, M. Ruben, and H. S. J. van der Zant, 2016, J. Am. Chem. Soc. 138, 8465.

Onida, G., L. Reining, and A. Rubio, 2002, Rev. Mod. Phys. 74, 601. Orrit, M., T. Ha, and V. Sandoghdar, 2014, Chem. Soc. Rev. 43, 973.

Osorio, E. A., K. Moth-Poulsen, H. S. J. van der Zant, J. Paaske, P. Hedegård, K. Flensberg, J. Bendix, and T. Bjørnholm, 2010, Nano Lett. 10, 105.

Osorio, E. A., K. O’Neill, N. Stuhr-Hansen, O.F. Nielsen, T. Bjørnholm, and H. S. J. van der Zant, 2007, Adv. Mater. 19, 281. Osorio, E. A., K. O’Neill, M. Wegewijs, N. Stuhr-Hansen, J. Paaske, T. Bjørnholm, and H. S. J. van der Zant, 2007, Nano Lett. 7, 3336. Ozaki, T., K. Nishio, and H. Kino, 2010, Phys. Rev. B 81, 035116.

Paaske, J., A. Rosch, P. Wölfle, C. M. Marcus, and J. Nygård, 2006, Nat. Phys. 2, 460.

Palacios, J. J., A. J. Pérez-Jiménez, E. Louis, and J. A. Vergés, 2001, Phys. Rev. B 64, 115411.

Papior, N., N. Lorente, T. Frederiksen, A. Garcia, and M. Brandbyge, 2017, Comput. Phys. Commun. 212, 8.

Park, H., A. K. L. Lim, A. P. Alivisatos, J. Park, and P. L. McEuen, 1999, Appl. Phys. Lett. 75, 301.

Park, J., et al., 2002, Nature (London) 417, 722.

Park, Y. S., A. C. Whalley, M. Kamenetska, M. L. Steigerwald, M. S. Hybertsen, C. Nuckolls, and L. Venkataraman, 2007, J. Am. Chem. Soc. 129, 15768.

Parker, C. R., et al., 2014, J. Am. Chem. Soc. 136, 16497.

Parks, J. J., A. R. Champagne, G. R. Hutchison, S. Flores-Torres, H. D. Abruna, and D. C. Ralph, 2007, Phys. Rev. Lett. 99, 026601.

Parks, J. J., et al., 2010, Science 328, 1370.

Pascual, J. I., J. Méndez, J. Gómez-Herrero, A. M. Baró, N. García, and V. T. Binh, 1993, Phys. Rev. Lett. 71, 1852.

Pasupathy, A. N., R. C. Bialczak, J. Martinek, J. E. Grose, L. A. Donev, P. L. McEuen, and D. C. Ralph, 2004, Science 306, 86.

Paulsson, M., and S. Datta, 2003, Phys. Rev. B 67, 241403(R).

Paulsson, M., T. Frederiksen, and M. Brandbyge, 2005, Phys. Rev. B 72, 201101(R).

Paulsson, M., T. Frederiksen, and M. Brandbyge, 2006, Nano Lett. 6, 258.

Paulsson, M., T. Frederiksen, H. Ueba, N. Lorente, and M. Brandbyge, 2008, Phys. Rev. Lett. 100, 226604. 
Paulsson, M., C. Krag, T. Frederiksen, and M. Brandbyge, 2009, Nano Lett. 9, 117.

Pauly, F., J. K. Viljas, U. Huniar, M. Häfner, S. Wohlthat, J. Bürkle, J. C. Cuevas, and G. Schön, 2008, New J. Phys. 10, 125019.

Pecchia, A., and A. DiCarlo, 2004, Rep. Prog. Phys. 67, 1497.

Pedersen, K. G. L., A. Borges, P. Hedegard, G. C. Solomon, and M. Strange, 2015, J. Phys. Chem. C 119, 26919.

Pedersen, K. G. L., M. Strange, M. Leijnse, P. Hedegard, G. C. Solomon, and J. Paaske, 2014, Phys. Rev. B 90, 125413.

Perdew, J. P., and M. Levy, 1983, Phys. Rev. Lett. 51, 1884.

Perdew, J. P., R. G. Parr, M. Levy, and J. L. Balduz, 1982, Phys. Rev. Lett. 49, 1691.

Perrin, M. L., E. Galan, R. Eelkema, F. C. Grozema, J. M. Thijssen, and H. S. J. van der Zant, 2015, J. Phys. Chem. C 119, 5697.

Perrin, M. L., E. Galan, R. Eelkema, J. M. Thijssen, F. C. Grozema, and H. S. J. van der Zant, 2016, Nanoscale 8, 8919.

Perrin, M. L., C. J. O. Verzijl, C. A. Martin, A. J. Shaikh, R. Eelkema, J. H. van Esch, J. M. van Ruitenbeek, J. M. Thijssen, H. S. J. van der Zant, and D. Dulić, 2013, Nat. Nanotechnol. 8, 282.

Perrin, M. L., et al., 2014, Nat. Nanotechnol. 9, 830.

Pickup, B. T., and P. W. Fowler, 2008, Chem. Phys. Lett. 459, 198.

Pientka, F., L. I. Glazman, and F. von Oppen, 2013, Phys. Rev. B 88, 155420.

Plihal, M., and J. W. Gadzuk, 2001, Phys. Rev. B 63, 085404.

Posazhennikova, A., and P. Coleman, 2005, Phys. Rev. Lett. 94, 036802.

Proetto, C., and A. López, 1981, Phys. Rev. B 24, 3031.

Pruschke, T., and N. Grewe, 1989, Z. Phys. B 74, 439.

Qiu, X. H., G. V. Nazin, and W. Ho, 2003, Science 299, 542.

Quek, S. Y., H. J. Choi, S. G. Louie, and J. B. Neaton, 2009, Nano Lett. 9, 3949.

Quek, S. Y., L. Venkataraman, H. J. Choi, S. G. Louie, M. S. Hybertsen, and J. B. Neaton, 2007, Nano Lett. 7, 3477.

Quinn, J. R., F. W. Foss, L. Venkataraman, M. S. Hybertsen, and R. Breslow, 2007, J. Am. Chem. Soc. 129, 6714.

Rabache, V., J. Chaste, P. Petit, M. L. D. Rocca, P. Martin, J.-C. Lacroix, R. L. McCreery, and P. Lafarge, 2013, J. Am. Chem. Soc. 135, 10218.

Rai, D., and M. Galperin, 2012, Phys. Rev. B 86, 045420.

Rai, D., O. Hod, and A. Nitzan, 2010, J. Phys. Chem. C 114, 20583.

Rai, D., O. Hod, and A. Nitzan, 2011, J. Phys. Chem. Lett. 2, 2118.

Rai, D., O. Hod, and A. Nitzan, 2012, Phys. Rev. B 85, 155440.

Rakhmilevitch, D., R. Korytár, A. Bagrets, F. Evers, and O. Tal, 2014, Phys. Rev. Lett. 113, 236603.

Rascón-Ramos, H., J. M. Artés, Y. Li, and J. Hihath, 2015, Nat. Mater. 14, 517.

Ray, S. G., S. S. Daube, G. Leitus, Z. Vager, and R. Naaman, 2006, Phys. Rev. Lett. 96, 036101.

Reddy, P., S.-Y. Jang, R. A. Segalman, and A. Majumdar, 2007, Science 315, 1568.

Reecht, G., H. Bulou, F. Scheurer, V. Speisser, F. Mathevet, C. González, Y. J. Dappe, and G. Schull, 2015, J. Phys. Chem. Lett. 6, 2987.

Reecht, G., C. Lotze, D. Sysoiev, T. Huhn, and K. Franke, 2016, ACS Nano 10, 10555.

Reecht, G., F. Scheurer, V. Speisser, Y. J. Dappe, F. Mathevet, and G. Schull, 2014, Phys. Rev. Lett. 112, 047403.

Reed, M. A., C. Zhou, C. J. Muller, T. P. Burgin, and J. M. Tour, 1997, Science 278, 252.

Reichert, J., R. Ochs, D. Beckmann, H. B. Weber, M. Mayor, and H. von Löhneysen, 2002, Phys. Rev. Lett. 88, 176804.

Reichert, J., H. B. Weber, M. Mayor, and H. von Löhneysen, 2003, Appl. Phys. Lett. 82, 4137.
Requist, R., S. Modesti, P. P. Baruselli, A. Smogunov, M. Fabrizio, and E. Tosatti, 2014, Proc. Natl. Acad. Sci. U.S.A. 111, 69.

Reuter, M. G., 2017, J. Phys. Condens. Matter 29, 053001.

Rincón-García, L., C. Evangeli, G. Rubio-Bollinger, and N. Agraït, 2016, Chem. Soc. Rev. 45, 4285.

Rix, J. B., and P. Hedegård, 2019, J. Phys. Chem. C 123, 3817.

Roch, N., S. Florens, V. Bouchiat, W. Wernsdorfer, and F. Balestro, 2008, Nature (London) 453, 633.

Roch, N., S. Florens, T. A. Costi, W. Wernsdorfer, and F. Balestro, 2009, Phys. Rev. Lett. 103, 197202.

Rocha, A. R., V. M. Garcia-Suarez, S. Bailey, C. Lambert, J. Ferrer, and S. Sanvito, 2006, Phys. Rev. B 73, 085414.

Romeike, C., M. R. Wegewijs, M. Ruben, W. Wenzel, and H. Schoeller, 2007, Phys. Rev. B 75, 064404.

Roura-Bas, P., and A. A. Aligia, 2010, J. Phys. Condens. Matter 22, 025602.

Ruben, M., A. Landa, E. Lörtscher, H. Riel, M. Mayor, H. Görls, H. B. Weber, A. Arnold, and F. Evers, 2008, Small 4, 2229.

Rzeznicka, I. I., J. Lee, P. Maksymovych, and J. T. Yates, 2005, J. Phys. Chem. B 109, 15992.

Sabater, C., C. Untiedt, and J. M. van Ruitenbeek, 2015, Beilstein J. Nanotechnol. 6, 2338.

Salomon, A., D. Cahen, S. Lindsay, J. Tomfohr, V. B. Engelkes, and C. D. Frisbie, 2003, Adv. Mater. 15, 1881.

Sangtarash, S., H. Sadeghi, and C. J. Lambert, 2016, Nanoscale 8, 13199.

Sangtarash, S., A. Vezzoli, H. Sadeghi, N. Ferri, H. M. O'Brian, I. Grace, L. Booffier, S. J. Higgins, R. J. Nichols, and C. J. Lambert, 2018, Nanoscale 10, 3060.

Scheer, E., and J.C. Cuevas, 2017, Molecular Electronics, An Introduction to Theory and Experiment (World Scientific, New Jersey).

Schinabeck, C., A. Erpenbeck, R. Härtle, and M. Thoss, 2016, Phys. Rev. B 94, 201407(R).

Schinabeck, C., R. Härtle, H. B. Weber, and M. Thoss, 2014, Phys. Rev. B 90, 075409.

Schinabeck, C., and M. Thoss, 2019, arXiv:1910.09577.

Schmaus, S., A. Bagrets, Y. Nahas, T. K. Yamada, A. Bork, M. Bowen, E. Beaurepaire, F. Evers, and W. Wulfhekel, 2011, Nat. Nanotechnol. 6, 185.

Schmid, D. R., S. Smirnov, M. Margańska, A. Dirnaichner, P. L. Stiller, M. Grifoni, A. K. Hüttel, and C. Strunk, 2015, Phys. Rev. B 91, 155435.

Schmidt, T. L., and A. Komnik, 2009, Phys. Rev. B 80, 041307.

Schmitteckert, P., R. Thomale, R. Korytár, and F. Evers, 2017, J. Chem. Phys. 146, 092320.

Schnäbele, P., 2014, master's thesis (Karlsruhe Institute of Technology).

Schoenauer, B., N. M. Gergs, P. Schmitteckert, F. Evers, and D. Schuricht, 2019, Phys. Rev. Research 1, 022006(R).

Schull, G., Y. J. Dappe, C. González, H. Bulou, and R. Berndt, 2011, Nano Lett. 11, 3142.

Schull, G., T. Frederiksen, A. Arnau, D. Sánchez-Portal, and R. Berndt, 2011, Nat. Nanotechnol. 6, 23.

Schwarz, F., M. Koch, G. Kastlunger, H. Berke, R. Stadler, K. Venkatesan, and E. Lörtscher, 2016, Angew. Chem., Int. Ed. Engl. 55, 11781.

Scott, G. D., and D. Natelson, 2010, ACS Nano 4, 3560.

Secker, D., S. Wagner, S. Ballmann, R. Härtle, M. Thoss, and H. B. Weber, 2011, Phys. Rev. Lett. 106, 136807.

Sedghi, G., et al., 2011, Nat. Nanotechnol. 6, 517.

Sek, S., A. Misicka, K. Swiatek, and E. Maicka, 2006, J. Phys. Chem. B 110, 19671. 
Sergueev, N., L. Tsetseris, K. Varga, and S. Pantelides, 2010, Phys. Rev. B 82, 073106.

Sham, L., and M. Schlüter, 1983, Phys. Rev. Lett. 51, 1888.

Shen, B., J. Tatchen, E. Sanchez-Gracia, and H. Bettinger, 2018, Angew. Chem., Int. Ed. Engl. 57, 10508.

Simmons, J. G., 1963, J. Appl. Phys. 34, 1793.

Smidstrup, S., et al., 2019, arXiv:1905.02794.

Smit, R. H. M., Y. Noat, C. Untiedt, N. D. Lang, M. C. van Hemert, and J. M. van Ruitenbeek, 2002, Nature (London) 419, 906.

Smith, C. E., S. O. Odoh, S. Ghosh, L. Gagliardi, C. J. Cramer, and C. D. Frisbie, 2015, J. Am. Chem. Soc. 137, 15732.

Solomon, G. C., D. Q. Andrews, R. H. Goldsmith, T. Hansen, M. R. Wasielewski, R. P. Van Dyne, and M. A. Ratner, 2008a, J. Am. Chem. Soc. 130, 17301.

Solomon, G. C., D. Q. Andrews, T. Hansen, R. H. Goldsmith, M. R. Wasielewski, R. van Duyne, and M. Ratner, 2008, J. Chem. Phys. 129, 054701.

Solomon, G. C., J. P. Bergfield, C. A. Stafford, and M. A. Ratner, 2011, Beilstein J. Nanotechnol. 2, 862.

Solomon, G. C., C. Herrmann, T. Hansen, V. Mujica, and M. A. Ratner, 2010, Nat. Chem. 2, 223.

Song, H., Y. Kim, Y. H. Jang, H. Jeong, M. A. Reed, and T. Lee, 2009, Nature (London) 462, 1039.

Song, H., Y. Kim, H. Jeong, M. A. Reed, and T. Lee, 2010, J. Phys. Chem. C 114, 20431.

Sowa, J. K., J. A. Mol, G. A. D. Briggs, and E. M. Gauger, 2018, J. Chem. Phys. 149, 154112.

Stadler, R., 2015, Nano Lett. 15, 7175.

Stefani, D., K. J. Weiland, M. Skripnik, C. Hsu, M. L. Perrin, M. Mayor, F. Pauly, and H. S. J. van der Zant, 2018, Nano Lett. 18, 5981.

Stefanucci, G., and S. Kurth, 2011, Phys. Rev. Lett. 107, 216401.

Stefanucci, G., and R.v. Leeuwen, 2013, Nonequilibrium Many-

Body Theory of Quantum Systems (Cambridge University Press, Cambridge, England).

Stipe, B. C., M. A. Rezaei, and W. Ho, 1998, Science 280, 1732.

Stokbro, K., J. Taylor, M. Brandbyge, J.-L. Mozos, and P. Ordejón, 2003, Comput. Mater. Sci. 27, 151.

Strachan, D. R., D. E. Johnston, B. S. Guiton, S. S. Datta, P. K. Davies, D. A. Bonnell, and A. T. C. Johnson, 2008, Phys. Rev. Lett. 100, 056805.

Strange, M., O. Lopez-Acevedo, and H. Häkkinen, 2010, J. Phys. Chem. Lett. 1, 1528.

Strange, M., J. S. Seldenthuis, C. j. L. Verzijl, J. M. Thijssen, and G. C. Solomon, 2015, J. Chem. Phys. 142, 084703.

Strange, M., and K. S. Thygesen, 2011, Beilstein J. Nanotechnol. 2, 746.

Stuyver, T., N. Blotwijk, S. Fias, P. Geerlings, and F. De Proft, 2017, ChemPhysChem 18, 3012.

Stuyver, T., S. Fias, F. De Proft, P. W. Fowler, and P. Geerlings, 2015, J. Chem. Phys. 142, 094103.

Stuyver, T., S. Fias, F. De Proft, P. Geerlings, Y. Tsuji, and R. Hoffmann, 2017, J. Chem. Phys. 146, 092310.

Su, T. A., H. Li, R. Klausen, N. Kim, M. Neupane, J. Leighton, M. L. Steigerwald, L. Venkataraman, and C. Nuckolls, 2017, Acc. Chem. Res. 50, 1088.

Su, T. A., H. Li, V. Zhang, M. Neupane, A. Batra, R. S. Klausen, B. Kumar, M. L. Steigerwald, L. Venkataraman, and C. Nuckolls, 2015, J. Am. Chem. Soc. 137, 12400.

$\mathrm{Su}$, T. A., M. Neupane, M. L. Steigerwald, L. Venkataraman, and C. Nuckolls, 2016, Nat. Rev. Mater. 1, 16002.

Su, W. P., J. R. Schrieffer, and A. J. Heeger, 1980, Phys. Rev. B 22, 2099.
Sýkora, and T. Novotný, 2017, J. Chem. Phys. 146, 174114.

Tada, T., and K. Yoshizawa, 2002, ChemPhysChem 3, 1035.

Tada, T., and K. Yoshizawa, 2015, Phys. Chem. Chem. Phys. 17, 32099.

Tal, O., M. Krieger, B. Leerink, and J. M. van Ruitenbeek, 2008, Phys. Rev. Lett. 100, 196804.

Taylor, H., X. Guo, and J. Wang, 2001, Phys. Rev. B 63, 245407.

Temirov, R., A. Lassise, F. B. Anders, and F. S. Tautz, 2008, Nanotechnology 19, 065401.

Tewari, S., 2018, Ph.D. thesis (Leiden University).

Tewari, S., C. Sabater, and J. van Ruitenbeek, 2019, Nanoscale 11, 19462.

Thiele, S., F. Balestro, R. Ballouo, S. Klyatskaya, M. Ruben, and W. Wernsdorfer, 2014, Science 344, 1135.

Thijssen, W. H. A., D. Djukic, A. F. Otte, R. H. Bremmer, and J. M. van Ruitenbeek, 2006, Phys. Rev. Lett. 97, 226806.

Thoss, M., and F. Evers, 2018, J. Chem. Phys. 148, 030901.

Thygesen, K. S., and K. W. Jacobsen, 2005a, Phys. Rev. Lett. 94, 036807.

Thygesen, K. S., and K. W. Jacobsen, 2005b, Chem. Phys. 319, 111. Thygesen, K. S., and A. Rubio, 2007, J. Chem. Phys. 126, 091101.

Thygesen, K. S., and A. Rubio, 2008, Phys. Rev. B 77, 115333.

Tivanski, A. V., Y. He, E. Borguet, H. Liu, G. C. Walker, and D. H. Waldeck, 2005, J. Phys. Chem. B 109, 5398.

Todorov, T. N., D. Dundas, J.-T. Lü, M. Brandbyge, and P. Hedegard, 2014, Eur. J. Phys. 35, 065004.

Todorov, T. N., J. Hoekstra, and A. P. Sutton, 2001, Phys. Rev. Lett. 86, 3606.

Toher, C., A. Filippetti, S. Sanvito, and K. Burke, 2005, Phys. Rev. Lett. 95, 146402.

Tour, J. M., L. Jones II, D. L. Pearson, J. J. S. Lamba, T. P. Burgin, G. M. Whitesides, D. L. Allara, A. N. Parikh, and S. V. Atre, 1995, J. Am. Chem. Soc. 117, 9529.

Troisi, A., and M. A. Ratner, 2006, Nano Lett. 6, 1784.

Tröster, P., P. Schmitteckert, and F. Evers, 2012, Phys. Rev. B 85, 115409.

Tsuji, Y., E. Estrada, R. Movassagh, and R. Hoffmann, 2018, Chem. Rev. 118, 4887.

Tsuji, Y., R. Hoffmann, R. Movassagh, and S. Datta, 2014, J. Chem. Phys. 141, 224311.

Tsukahara, N., S. Shiraki, S. Itou, N. Ohta, N. Takagi, and M. Kawai, 2011, Phys. Rev. Lett. 106, 187201.

Tsutsui, M., Y. Teramae, S. Kurokawa, and A. Sakai, 2008, Appl. Phys. Lett. 92, 029903.

Tu, M. W.-Y., A. Aharony, O. Entin-Wohlman, A. Schiller, and W.-M. Zhang, 2016, Phys. Rev. B 93, 125437.

Tu, M. W.-Y., W.-M. Zhang, J. Jin, O. Entin-Wohlman, and A. Aharony, 2012, Phys. Rev. B 86, 115453.

Ueda, A., Y. Utsumi, Y. Tokura, O. Entin-Wohlman, and A. Aharony, 2017, J. Chem. Phys. 146, 092313.

Újsághy, O., J. Kroha, L. Szunyogh, and A. Zawadowski, 2000, Phys. Rev. Lett. 85, 2557.

Ulčakar, L., T. Rejec, J. Kokalj, S. Sangtarash, H. Sadeghi, A. Ramšak, J. H. Jefferson, and C. J. Lambert, 2019, Sci. Rep. 9, 3478.

Ulrich, J., D. Esrail, W. Pontius, L. Venkataraman, D. Millar, and L. H. Doerrer, 2006, J. Phys. Chem. B 110, 2462.

Urban, D. F., R. Avriller, and A. Levy Yeyati, 2010, Phys. Rev. B 82, 121414(R).

Utsumi, Y., O. Entin-Wohlman, A. Ueda, and A. Aharony, 2013, Phys. Rev. B 87, 115407.

Valli, A., A. Amaricci, V. Brosco, and M. Capone, 2018, Nano Lett. 18, 2158. 
Valli, A., A. Amaricci, V. Brosco, and M. Capone, 2019, Phys. Rev. B 100, 075118.

van der Molen, S. J., J. Liao, T. Kudernac, J. S. Agustsson, L. Bernard, M. Calame, B. J. van Wees, B. L. Feringa, and C. Schönenberger, 2009, Nano Lett. 9, 76.

van der Zant, H. S. J., et al., 2006, Faraday Discuss. 131, 347.

van Dijk, E.H., D. J.T. Myles, M.H. van der Veen, and J.C. Hummelen, 2006, Org. Lett. 8, 2333.

van Houten, H., L. W. Molenkamp, C. W. J. Beenakker, and C. T. Foxon, 1992, Semicond. Sci. Technol. 7, B215.

van Ruitenbeek, J. M., A. Alvarez, I. Piñeyro, C. Grahmann, P. Joyez, M. H. Devoret, D. Esteve, and C. Urbina, 1996, Rev. Sci. Instrum. 67, 108 .

van Setten, M. J., D. Xenioti, M. Alouani, F. Evers, and R. Korytár, 2019, J. Phys. Chem. C 123, 8902.

van Setten, M. J., et al., 2015, J. Chem. Theory Comput. 11, 5665.

Varela, S., E. Medina, F. Lopez, and V. Mujica, 2014, J. Phys. Condens. Matter 26, 015008.

Varela, S., V. Mujica, and E. Medina, 2016, Phys. Rev. B 93, 155436. Varma, C. M., Z. Nussinov, and W. Van Saarloos, 2002, Phys. Rep. 361, 267.

Vázquez, H., R. Skouta, S. Schneebeli, M. Kamenetska, R. Breslow, L. Venkataraman, and M. S. Hybertsen, 2012, Nat. Nanotechnol. 7, 663.

Venkataraman, L., J.E. Klare, C. Nuckolls, M. S. Hybertsen, and M. L. Steigerwald, 2006, Nature (London) 442, 904.

Venkataraman, L., J. E. Klare, I. W. Tam, C. Nuckolls, M. S. Hybertsen, and M. L. Steigerwald, 2006, Nano Lett. 6, 458.

Vilan, A., 2007, J. Phys. Chem. C 111, 4431.

Vojta, M., 2006, Philos. Mag. 86, 1807.

Vojta, M., R. Bulla, and W. Hofstetter, 2002, Phys. Rev. B 65, 140405.

von Wrochem, F., D. Gao, F. Scholz, H.-G. Nothofer, G. Nelles, and J. M. Wessels, 2010, Nat. Nanotechnol. 5, 618.

Wagner, C., N. Fournier, F. S. Tautz, and R. Temirov, 2012, Phys. Rev. Lett. 109, 076102.

Walz, M., A. Bagrets, and F. Evers, 2015, J. Chem. Theory Comput. 11, 5161.

Walz, M., J. Wilhelm, and F. Evers, 2014, Phys. Rev. Lett. 113, 136602 .

Wang, C., A. S. Batsanov, M. R. Bryce, S. Martín, R. J. Nichols, S. J. Higgins, V. M. García-Suárez, and C. J. Lambert, 2009, J. Am. Chem. Soc. 131, 15647.

Ward, D. R., N. J. Halas, J. W. Ciszek, J. M. Tour, Y. Wu, P. Nordlander, and D. Natelson, 2008, Nano Lett. 8, 919.

Wegewijs, M. R., and K. C. Nowack, 2005, New J. Phys. 7, 239.

Weik, N., J. Schindler, S. Bera, G. C. Solomon, and F. Evers, 2016, Phys. Rev. B 94, 064204.

Welker, J., and F. Giessibl, 2012, Science 336, 444.

Wen, H.-M., Y. Yang, X.-S. Zhou, J.-Y. Liu, D.-B. Zhang, Z.-B. Chen, J.-Y. Wang, Z.-N. Chen, and Z.-Q. Tian, 2013, Chem. Sci. 4, 2471.

Widawsky, J. R., W. Chen, H. Vázquez, T. Kim, R. Breslow, M. S. Hybertsen, and L. Venkataraman, 2013, Nano Lett. 13, 2889.

Wierzbinski, E., and K. Slowinski, 2006, Langmuir 22, 5205.

Wilhelm, J., D. Golze, L. Talirz, J. Hutter, and C. A. Pignedoli, 2018,

J. Phys. Chem. Lett. 9, 306.

Wilhelm, J., and J. Hutter, 2016, J. Chem. Theory Comput. 12, 3623. Wilson, K. G., 1975, Rev. Mod. Phys. 47, 773.
Wu, S., M. T. González, R. Huber, S. Grunder, M. Mayor, C. Schönenberger, and M. Calame, 2008, Nat. Nanotechnol. 3, 569. Xia, J., et al., 2014, Nano Lett. 14, 2941.

Xia, J. L., I. Diez-Perez, and N. J. Tao, 2008, Nano Lett. 8, 1960.

Xiang, D., H. Jeong, T. Lee, and D. Mayer, 2013, Adv. Mater. 25, 4845.

Xiang, D., X. Wang, C. Jia, T. Lee, and X. Guo, 2016, Chem. Rev. 116, 4318.

Xiao, X., L. A. Nagahara, A. M. Rawlett, and N. Tao, 2005, J. Am. Chem. Soc. 127, 9235.

Xiao, X., B. Xu, and N. J. Tao, 2004, Nano Lett. 4, 267.

Xie, Z., T. Z. Markus, S. R. Cohen, Z. Vager, R. Guttierez, and R. Naaman, 2011, Nano Lett. 11, 4652.

Xing, Y., R.-H. Park, R. Venkatramani, S. Keinan, D. N. Beratan, M. J. Therien, and E. Borguet, 2010, J. Am. Chem. Soc. 132, 7946.

Xu, B., and N. J. Tao, 2003, Science 301, 1221.

Xu, B., X. Xiao, and N. J. Tao, 2003, J. Am. Chem. Soc. 125, 16164.

Xue, Y., and M. A. Ratner, 2003, Phys. Rev. B 68, 115406.

Yaliraki, S. N., A. E. Roitberg, C. Gonzalez, V. Mujica, and M. A. Ratner, 1999, J. Chem. Phys. 111, 6997.

Yamada, R., H. Kumazawa, T. Noutoshi, S. Tanaka, and H. Tada, 2008, Nano Lett. 8, 1237.

Yang, W., A. J. Cohen, and P. Mori-Sánchez, 2012, J. Chem. Phys. 136, 204111.

Yang, X., C. H. van der Wal, and B. J. van Wees, 2019, Phys. Rev. B 99, 024418.

Yeganeh, S., M. A. Ratner, E. Medina, and V. Mujica, 2009, J. Chem. Phys. 131, 014707.

Yelin, T., R. Korytár, N. Sukenik, R. Vardimon, B. Kumar, C. Nuckolls, F. Evers, and O. Tal, 2016, Nat. Mater. 15, 444.

Yoshizawa, K., T. Tada, and A. Staykov, 2008, J. Am. Chem. Soc. 130, 9406.

Yu, P., N. Kocić, J. Repp, B. Siegert, and B. Donarini, 2017, Phys. Rev. Lett. 119, 056801

Zeng, C., B. Li, B. Wang, H. Wang, K. Wang, J. Yang, J. Hou, and Q. Zhu, 2002, J. Chem. Phys. 117, 851.

Zhang, G. P., Z. Xie, Y. Song, G.-C. Hu, and C.-K. Wang, 2017, Top. Curr. Chem. 375, 85.

Zhang, L., et al., 2015, Phys. Rev. B 91, 195424.

Zhang, Y.-h., S. Kahle, T. Herden, C. Stroh, M. Mayor, U. Schlickum, M. Ternes, P. Wahl, and K. Kern, 2013, Nat. Commun. 4, 2110.

Zhao, A., et al., 2005, Science 309, 1542.

Zhao, X., V. Geskin, and R. Stadler, 2017, J. Chem. Phys. 146, 092308.

Zheng, J., et al., 2018, Chem. Sci. 9, 5033.

Zheng, J.-T., et al., 2016, Electrochim. Acta 200, 268.

Zhou, Y.X., F. Jiang, H. Chen, R. Note, H. Mizuseki, and Y. Kawazoe, 2008, J. Chem. Phys. 128, 044704.

Žitko, R., and J. Bonča, 2006, Phys. Rev. B 74, 045312.

Žonda, M., O. Stetsovych, R. Korytár, M. Ternes, R. Temirov, A. Racanelli, F. S. Tautz, P. Jelínek, T. Novotný, and M. Švec, 2018, arXiv:1811.00351.

See Supplemental Material at http://link.aps.org/supplemental/10 .1103/RevModPhys.92.035001 for three tables providing an overview of the experimental data from the literature for the conductance of Au-benzendithiol-Au, Au-alkanedithiol-Au, and $\mathrm{Au}-$ benzendiamine-Au single-molecule junctions. 